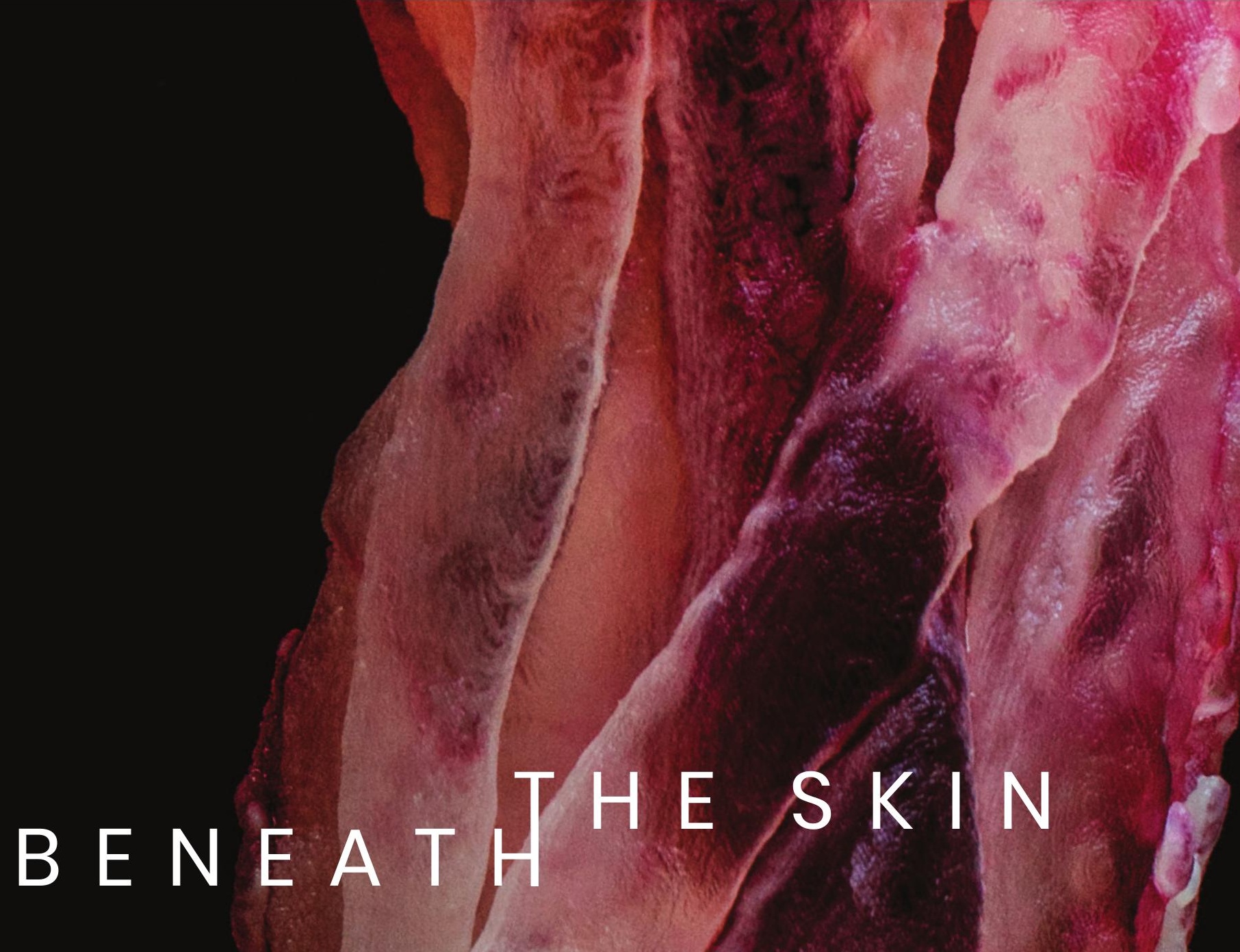

Emulating human physiology using a novel bitmap-based "voxel" 3D-printing workflow. 
Cover:

Figure 1. Close-up of bitmap-based 3D-printed neonatal limb (leg). 


\title{
BENEATH THE SKIN
}

by

A N A MOR R IS

\begin{abstract}
A 90-point research portfolio submitted to the Victoria University of Wellington in fulfilment of the requirements for the degree of Master of Design Innovation.
\end{abstract}

Primary Supervisor: Bernard Guy Secondary Supervisor: Ross Stevens 


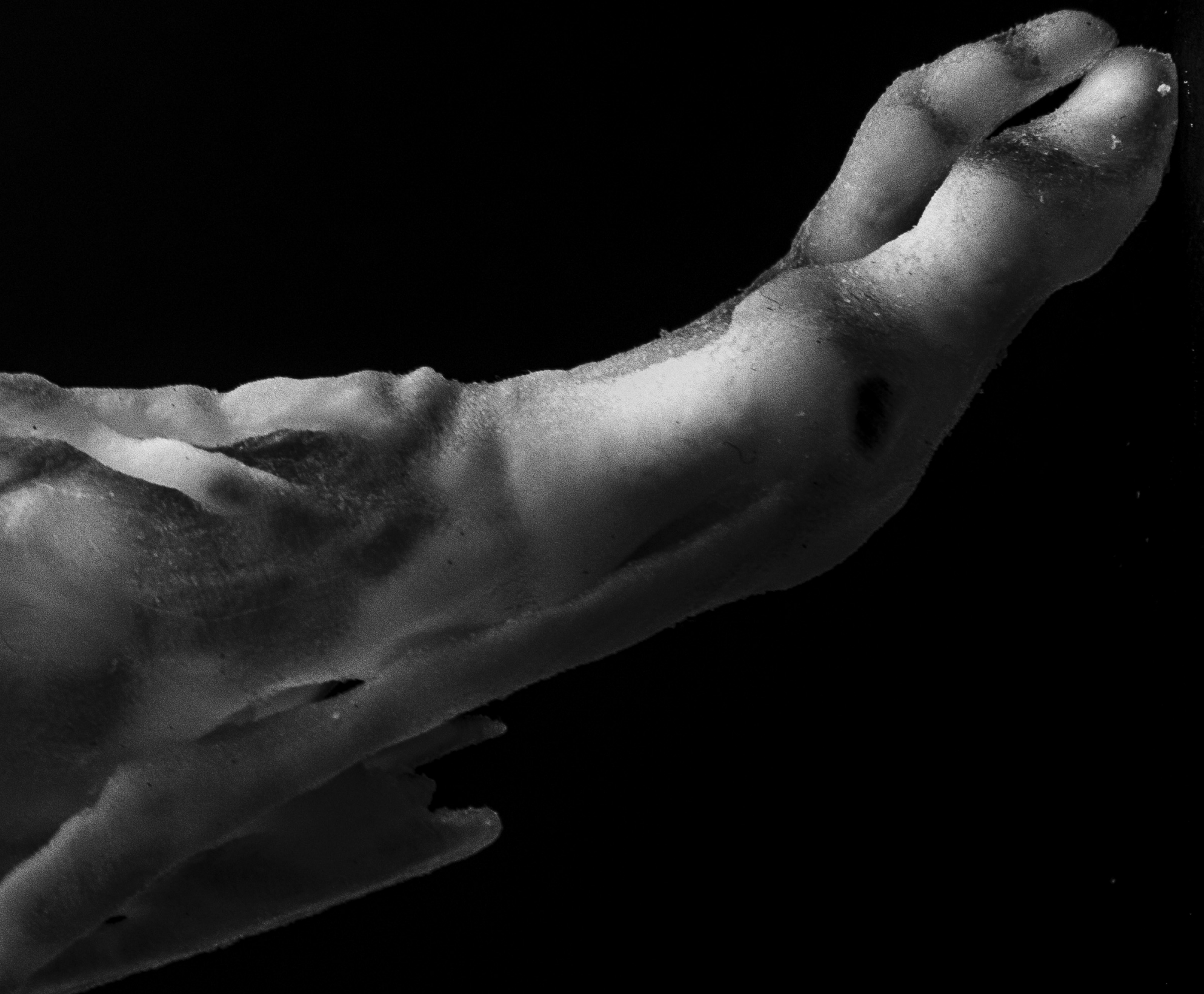




\section{DEDICATION}

To my Nana, Mary Morris

Left:

Figure 2. A bitmap-based 3D-printed foot. A close-up of the toe flexing. 


\section{Abstract}

Novel technologies that produce medical models which are synthetic equivalents to human tissue may forever change the way human anatomy and medicine are explored. Medical modelling using a bitmapbased additive manufacturing workflow offers exciting opportunities for medical education, informed consent practices, skills acquisition, pre-operative planning and surgical simulation. Moving medical data from the 2D-world to tactile, highly detailed 3D-printed anatomical models may significantly change how we comprehend the body; revamping everything - from medical education to clinical practice.

\section{Research Problem}

The existing workflow for producing patient-specific anatomical models from biomedical imaging data involves image thresholding and iso-surface extraction techniques that result in surface meshes (also known as objects or parts). This process restricts shape specification to one colour and density, limiting material blending and resulting in anatomically inequivalent medical models. So, how can the use of 3D-printing go beyond static anatomical replication? Imagine pulling back the layers of tissue to reveal the complexity of a procedure, allowing a family to understand and discuss their diagnosis. Overcoming the disadvantages of static medical models could be a breakthrough in the areas of medical communication and simulation. Currently, patient specific models are either rigid or mesh-based and, therefore, are not equivalents of physiology.

\section{Research Aim}

The aim of this research is to create tangible and visually compelling patient-specific prototypes of human anatomy, offering an insight into the capabilities of new bitmap-based 3D-printing technology. It proposes that full colour, multi-property, voxel-based 3D-printing can emulate physiology, creating a new format of visual and physical medical communication.

\section{Data Collection and Procedure}

The work explored and in this research portfolio is the first of its kind, entering into a 3D-printing territory that has never been published before. For this study, biomedical imaging data was converted into multiproperty 3D-printed synthetic anatomy by bypassing the conversion steps of traditional segmentation. Bitmap-based 3D-printing allows for the precise control over every 14-micron material droplet or "voxel". Control over each voxel involves a process of sending bitmap images to a high-resolution and multi-property 3D-printer. Bitmap-based 3D-printed synthetic medical models - which mimicked the colour and density of human anatomy - were successfully produced.

\section{Findings}

This research presented a novel and streamlined bitmap-based medical modelling workflow with the potential to save manufacturing time and labour cost. Moreover, this workflow produced highly accurate models with graduated densities, translucency, colour and flexion - overcoming complexities that arise due to our body's opaqueness. The presented workflow may serve as an incentive for others to investigate bitmap-based 3D-printing workflows for different manufacturing applications.

This designed definition may serve as an incentive for others to go beneath the skin and touch upon the essence of existence. 


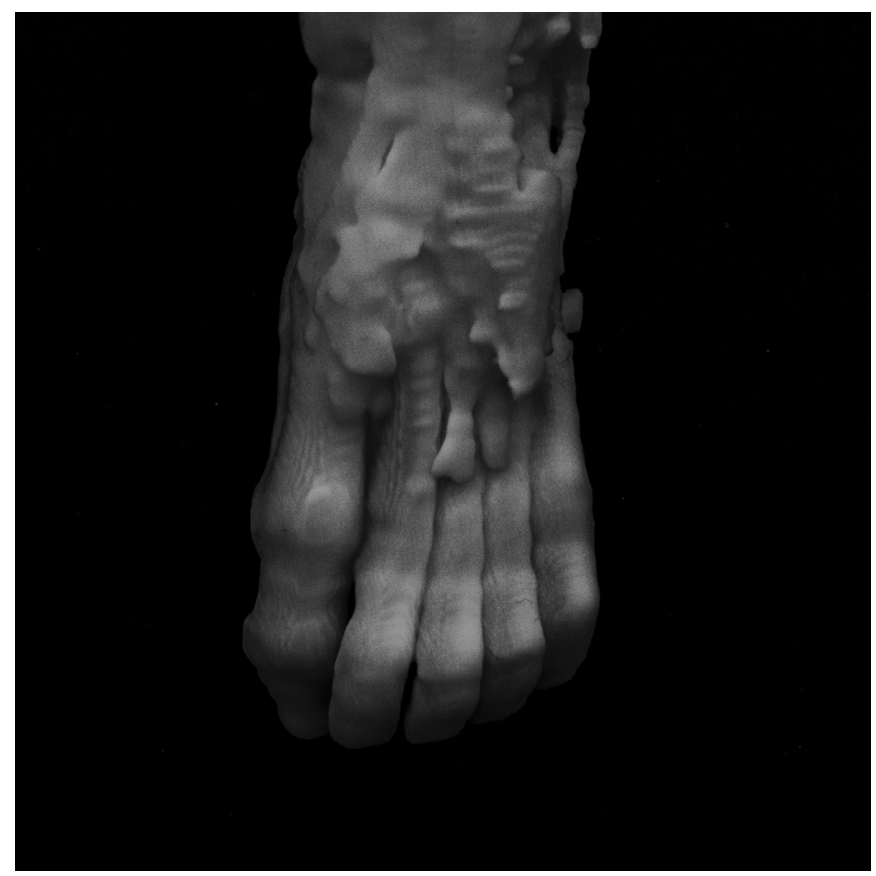

Figure 3. Image of bitmap-based 3D-printed neonatal toes. 
"With kindred presumptions of benefice, the doctor studies the body to improve its fate; the artist to improve its spirit.

Above all, these students start with the same genre of book: the illustrated anatomy. Here the body is laid bare inside and out in pictures that work in several realms.

They are science in subject, illustration in usefulness, but art in their potential emotional impact"

(Rifkin et al., 2006, p. 7). 


\section{Personal Motivation}

Throughout my Industrial Design Undergraduate Degree at the School of Design Innovation at Victoria University of Wellington, I had the privilege of being able to work on many multi-property 3D-printing projects. This undergraduate research led on to a Summer Scholarship research opportunity developing 3D-printed test and calibration phantoms for X-ray CT imaging in collaboration with the Department of Radiation Therapy, University of Otago Wellington and MARS Bioimaging, New Zealand (MBI). As a result, the summer research project taught me about the principles of biomedical imaging. This research portfolio presents a creative exploration that lies in the intersection between medical imaging principles, new technology and design. 


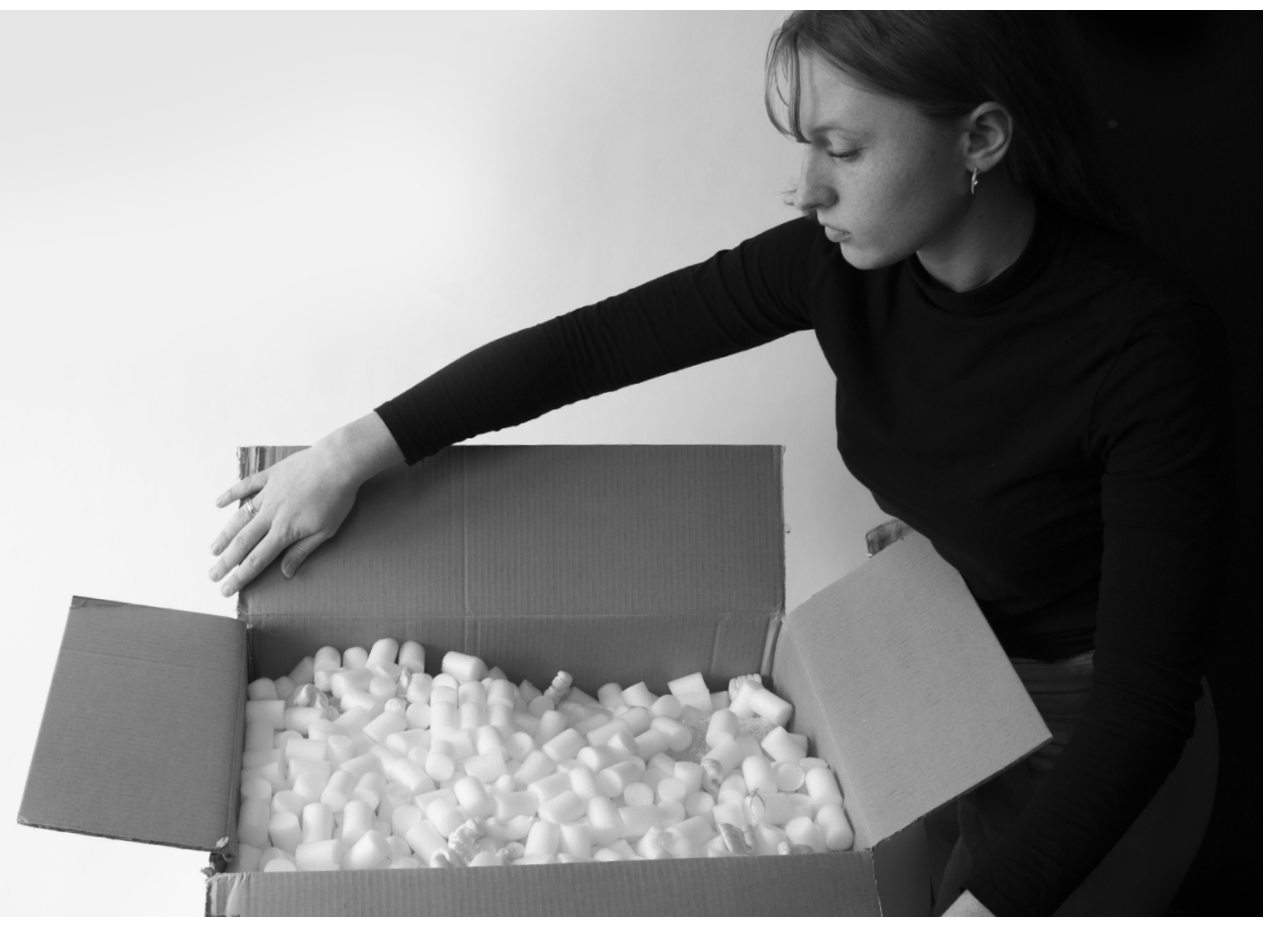

Figure 4. Unboxing 3D-prints sent from Stratasys in Shanghai, China. 


\section{Acknowledgements}

This research portfolio would not have been possible without all the people who have helped me throughout the way, and because of this, I will be forever grateful. 
- my primary supervisor, Bernard Guy, for everything you have done to help guide me throughout this process. This research would not have been possible without your encouragement.

- my secondary supervisor, Ross Stevens, for your support and guidance.

- Phil Jarret and Keith Davies, for all the help on the Stratasys J750 3D-Printer.

- MedTech CoRE and the MADE research stream for the generous research grant and support that made this research possible.

- other Victoria University of Wellington staff and research committee members.

- Dr. Ali Mirjalili, Dr. Alys Clark, Dr. Merryn Tawhai, Dr. Kelvin Woon, the team at Stratasys and to all the other people who have taken the time to give me some advice.

- the 2019/2020 Master of Design Innovation cohort, especially my close friends.

- my friends, for your continued understanding and support.

- my dog, for the cuddles.

- my family - especially my mum, dad and brother - 2020 has not been easy, but together we make a great team.

- Josh, a simple acknowledgement could never be enough to say thank you for everything you have helped me with - you're the best. 


\section{TABLE OF CONTENTS}

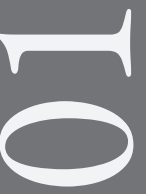

Dedication / IV

Abstract/VI

Preface / VIII

Personal Motivation / IX

Acknowledgements /XI

\section{NTROD U C T I O N}

1. 1 Chapter Content Descriptions / 2

1. 2 Terminology

1.2.1 Digital Fabrication Terminology / 4

1.1.1 Medical Terminology / 6

1. 3 Introduction to the Research / 11

1. 4 Research Direction / 14

1.4.1 Purpose Statement

1.4.2 Research Question

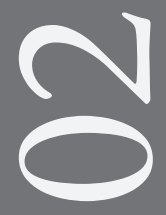

METHODOLO G Y

2. 1 Research Aims and Objectives / 18

2. 2 Research Methodologies / 21

2.2.1 Research Structure / 23

2.2.2 Practical Action Research / 24

2. 3 Research Methods / 24

2. 4 Software, Hardware and Data / 28

2.4.13D-Printers / 30

2.4.2 Programmes / 32

2.4.3 Data \& Ethics / 33

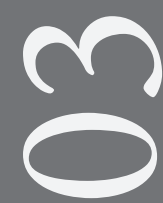

B A C K G R U N D RESEAR C H

3. 1 Historical Overview / 39

3. 2 Literature Review / 43

3.2.1 Biomedical Imaging / 44

3.2.2 Physical Modelling in Medicine / 47

3.2.3 Multi-Material 3D-Printing / 62

3.2.4 Project Scope / 68 


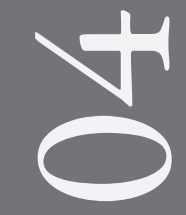

PRACTICAL ACTION RESEAR C H

4. 2 Case Study 1 / 76

4.1.1 Overview / 79

4.1.2 Criteria / 80

4.1.3 File Preparation / 81

4.1.4 Design Experimentation / 85

4.1.5 Final Design / 99

4.1.6 Reflection / 114

4. 3 Case Study $2 / 119$

4.2.1 Overview / 121

4.2.2 Criteria / 122

4.2.3 File Preparation / 123

4.2.4 Design Experimentation / 127

4.2.5 Design Refinement / 149

4.2.6 Final Design / 165

4.2.7 Reflection / 179

4.4 Case Study 3 / 183

4.3.1 Overview / 184

4.3.2 Criteria / 185

4.3.3 Material Exploration / 189

4.3.4 Design Experimentation / 199

4.3.5 Reflection / 201

4.3.6 DAP Voxel Software Concept / 213

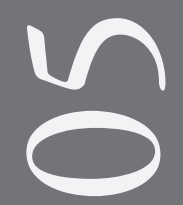

D I S C USS I O N

C O NCLUS I O N

5. 1 Key Findings / 219

6. 1 Concluding Statement / 241

5. 2 Interpretations / 222

6. 2 Reference List / 242

5.2.1 Case Study $1 / 222$

5.2.2 Case Study $2 / 224$

6.3 Appendix A / 250

5.2.3 Case Study 3 / 227

6. 4 Appendix B / 256

5. 3 Implications

5.3.1 Bitmap-based vs. Mesh-based 3D-Printing / 229

5.3.2 3D-Printing Theory and Practice/ 234

5.3.3 Cultural Implications / 235

5. 4 Limitations / 236

5. 5 Recommendations / 237 


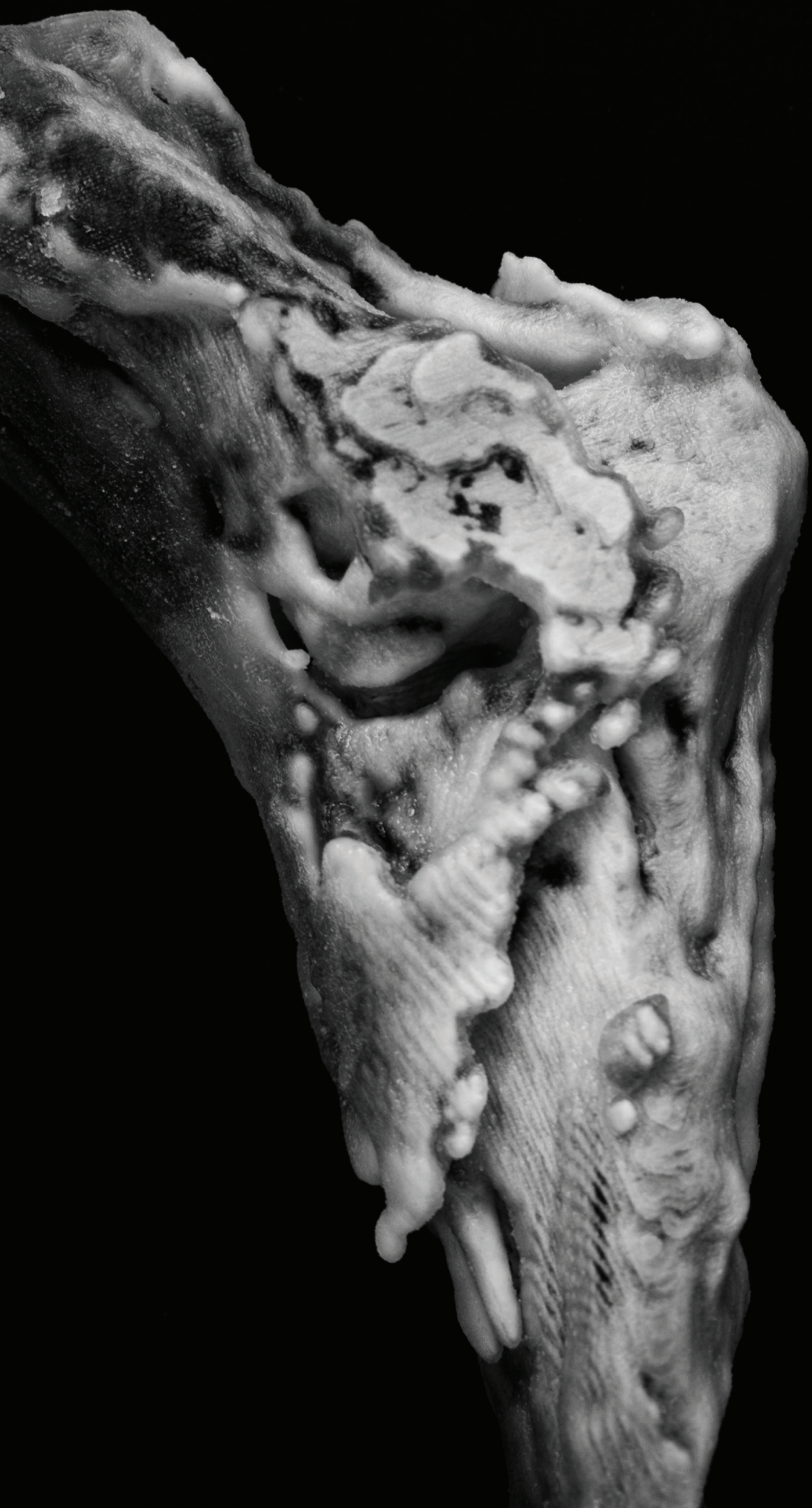




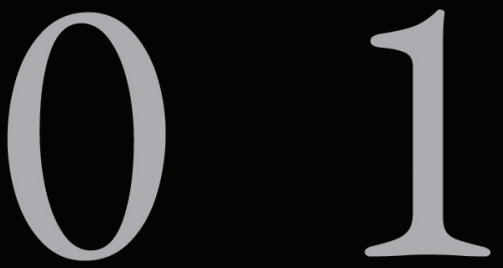

I N T RODUCTION

Figure 5. Bitmap-based 3D-printed neonatal foot (left). 


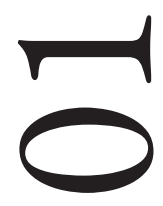

I NTRODUCTION

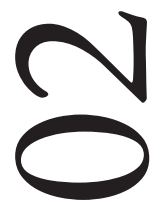

METHODOLOG Y

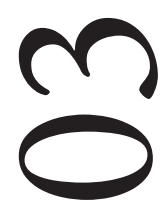

B A C K G R O N D R E S E A R C H
A brief introduction outlining the This section outlines how the research was project and the issue under investigation (introducing the reader to the topic). undertaken using the Research through Design methodology and a criteria-based approach. This chapter describes the research procedures, structure, software, hardware, aims, objectives and methods.
The Background Research section sets the scene and contextualises the research. A Historical Overview and a review of literature will support the research by analysing past studies and identifying gaps in the knowledge to help shape the research. Themes explored in this chapter include the history of anatomical studies, the value of 3D-printing in medicine and current 3D-printing workflows. 


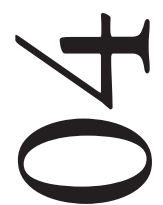

PRACTICALACTION R E S E A R C H

This chapter focusses on developing primary research through a creative and iterative design research process.

A Case Study approach was used in this section to show three avenues of design exploration. The three case studies are outlined in chronological order and become more refined based on feedback from expert opinions and qualitative analysis of design experiments.

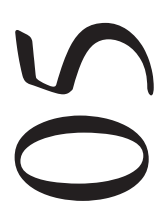

D I S C US SION

Included within the Discussion section is a critical analysis of the results, including the underlying meaning of the research, possible implications, improvements, limitations of the study, and whether the results successfully address the research criteria

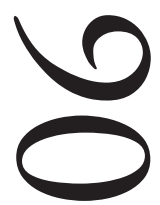

C O NCLUSION

The concluding section of this research summarises the key points in the research and highlights the research value by reiterating the main points, the research problem, results, findings, and future avenues for exploration. 
1. 2.1

Digital Fabrication Terminology

\section{D - P R I N T I N G}

a type of additive manufacturing (AM) that builds a physical object out of a digital blueprint by depositing sequential two-dimensional (2D) layers of material in order to create a three-dimensional (3D) form (Bastawrous et al., 2018, p. 2809).

\section{B I T M A P}

a type of image (e.g. PNG or JPEG file formats) that is made up of squares, or 'pixels', that form a grid making up a full image. The smaller the squares that make up an image, the higher the quality (resolution) of the image. Each pixel holds colour data.

B IT M A P - B A SED “V OXEL” 3 D - PR INTING

a method of $A M$ that can translate information from various types of bitmap datasets into heterogeneous physical entities by converting on-screen pixels into multi-material droplets. These micro-droplets are also referred to as 'voxels' (Bader et al., 2018, p. 2).

\section{VOXE L}

a term used to describe a '3D-printed pixel', a 14-micron material droplet.

\section{THE STRATASYS J 750 тM 3 D PRINTER}

a full-colour and multi-material Polyjet 3D-printer manufactured by Stratasys and supplied by the School of Design Innovation, Victoria University of Wellington.

\section{A G IL U S 30 тм}

a rubber-like PolyJet photopolymer material that can flex and bend.

\section{E R O TM}

a rigid PolyJet photopolymer material available in many colours.

\section{SHORE HARDNESS SCALE}

a scale which measures the 'hardness' (density) of rubber and plastic materials. 


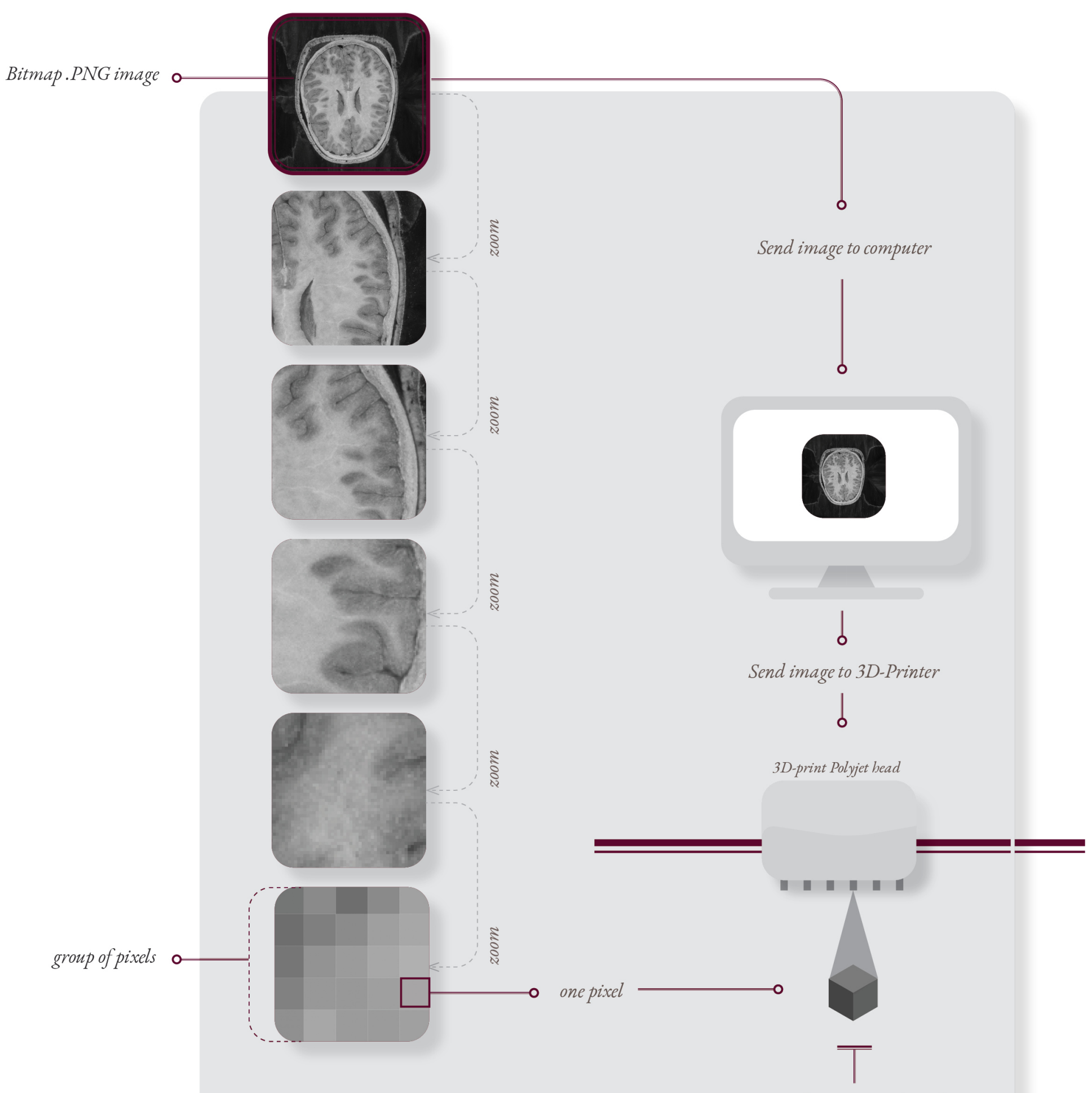

Voxel-3D-printed pixel

Figure 6. Bitmap to voxel process. 
1. 2.2

Medical Terminology

BIOMEDICAL IMAGING TECHNIQUES

are also known as "volumetric scanning technologies", which include computed tomography (CT) and Magnetic Resonance Imaging (MRI) (Doubrovski et al., 2015, p. 9).

\section{SE G M EN T A T I O N}

the process of extracting elements of an image into different parts such as different tissue classes, organs, and structures.

MEDICAL MODELLING (alsoknown as Bio-Fabrication) a term that refers to the use of biomedical imaging datasets to produce computeraided design (CAD) models, resulting in personalised medical devices or exact replicas of a patient's anatomy (Allenby \& Woodruff, 2019, p. 161). 


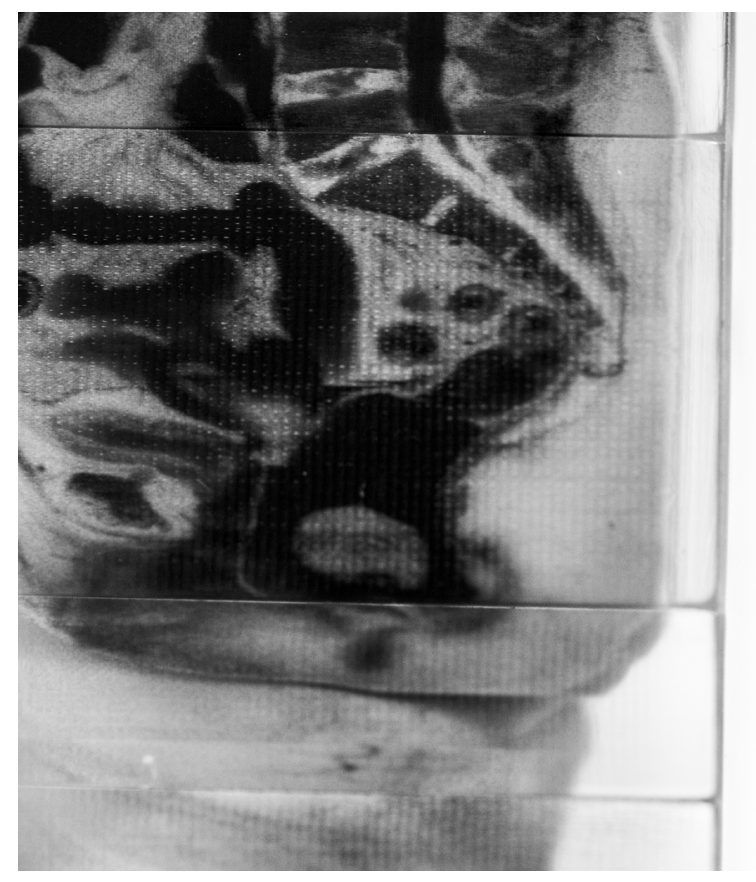

Figure 7. Details of bitmap-based 3D-printed Visible Human ${ }^{\circledR}$. 


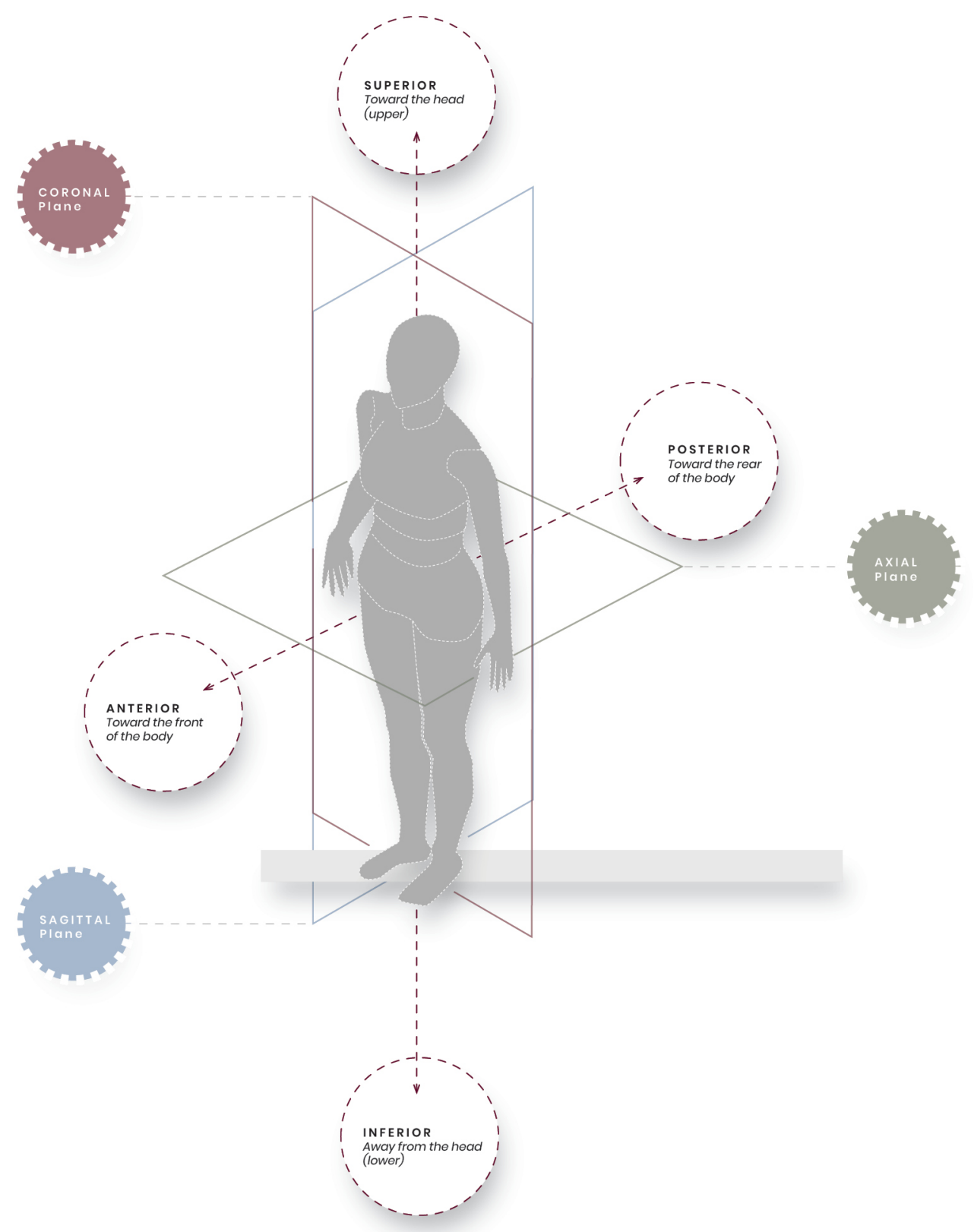




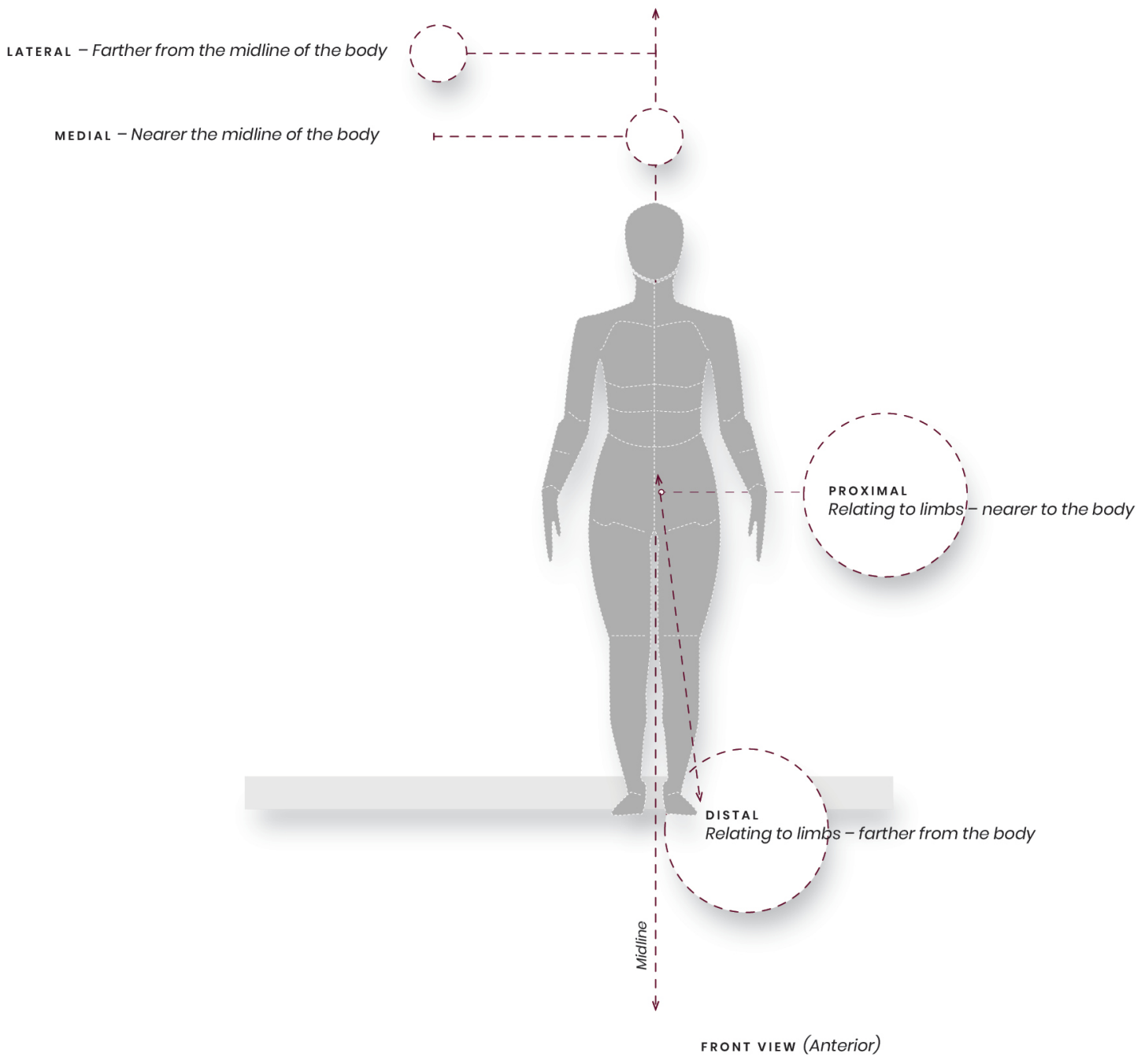

Figure 8. Anatomical directional terms. 


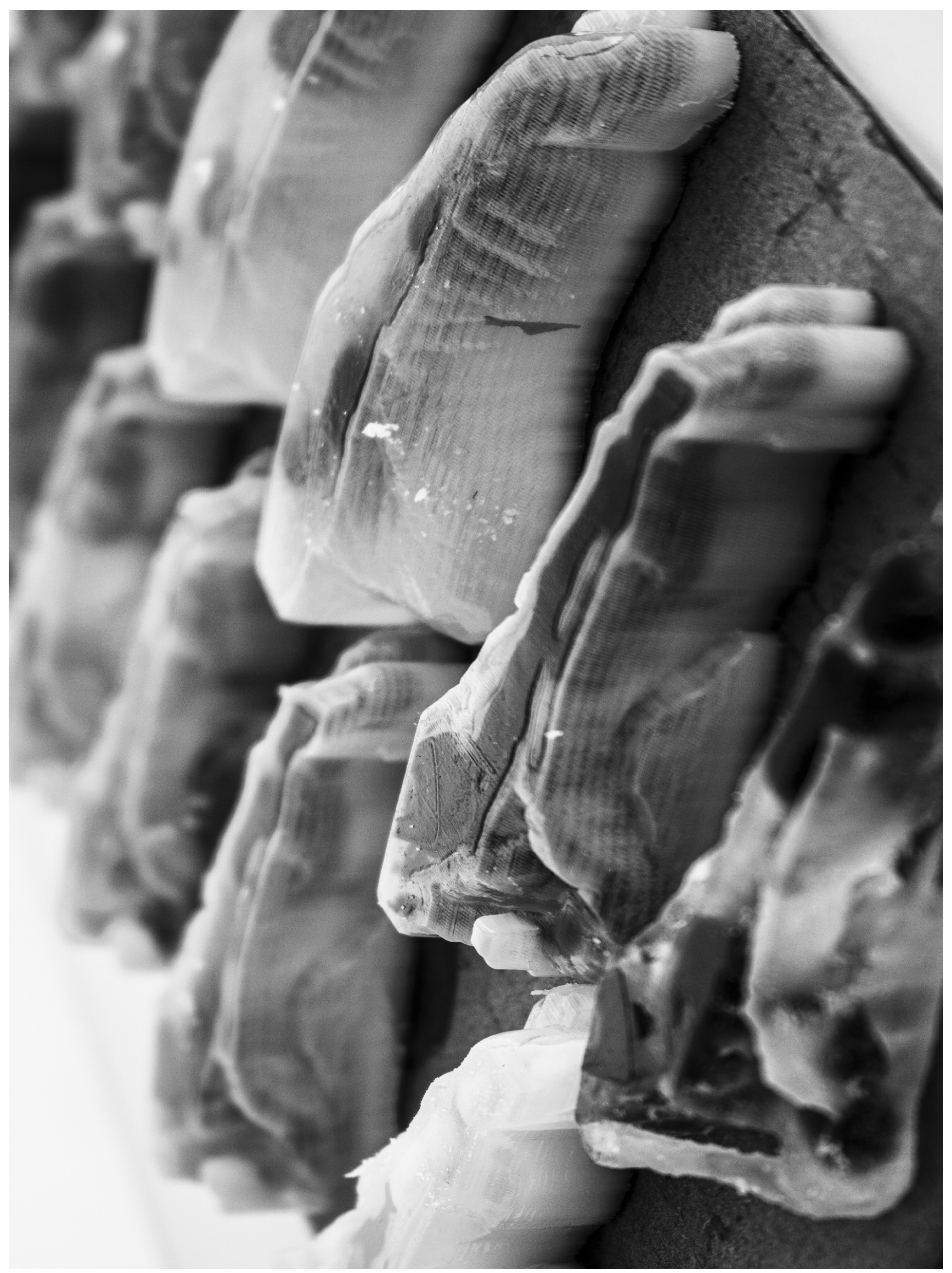

Figure 9. Growth through 3D-printing. 
CHAPTER I

\subsection{N T RO D U C T IO N}

FOR MILLENNIA, HOMO-SAPIENS HAVE BEEN FASCINATED BY WHAT LIES BENEATH THE SKIN. This curiosity has resulted in a constant evolution of technology and medical knowledge - providing for an ever-increasing understanding of the human body and its various intricacies.

Despite the wealth of knowledge available about the human body, it is still difficult to truly visualise what lies under the skin and how the body functions. To some degree, the human body has a mind of its own. Lungs instinctively fill with air; a baby will swallow without being told. Because of the body's automatic nature, there is immediate and justified cause for concern when the human body momentarily malfunctions. Clinicians question the cause of the malfunction, along with how it can be remedied.

Currently, clinicians rely on their use of tactile interaction with anatomy, feeling the range of textures and densities beneath the skin's surface. But, imagine if skin were transparent; if we could see underneath it, and watch a heart fuel our organs while they work away like little machines.

It is this possibility that spurred this research. 
Topic

The purpose of this research is to 3D-print patient-specific and high-fidelity medical models on the Stratasys J750 ${ }^{\mathrm{TM}}$ 3D-Printer using a bitmap-based 3D-printing workflow and biomedical imaging data. This will ultimately expose the potential of bitmap-based 3D-printing for the purpose of replicating anatomical density and colour in patient-specific medical models by engineering complex material combinations, controlling every 14-micron voxel. Harnessing novel medical modelling techniques may provoke a newfound way of imaging the human body - allowing for a whole new dimension of medial visualisation.

\section{Research Problem}

3D-printed medical models are currently used in the medical field in areas including surgical simulation, pre-operative planning and education. These models are created from biomedical imaging data to allow the production of exact replications of patient-specific anatomy time and time again, without the need to access anatomically inequivalent cadavers. Current medical models are often low-fidelity and do not accurately reflect the intricacies of the human body to a high degree of realism.

A justification of, or the importance of, the problem as found in the past research and in practice.

At the beginning of 2019, the high-resolution Stratasys J750 TM 3D-Printer became available to use at the School of Design Innovation at the Victoria University of Wellington. The opportunity to use this 3D-printing technology and its manufacturing capabilities drew interest. Notably, the capability to 3D-print in a bitmap-based format referred to by Stratasys as their "voxel printing” capability.

Bitmap-based 3D-printing is novel. Literature suggests that a bitmap-based medical modelling workflow has the potential to create medical models at a quicker rate and at a higher-resolution than traditional medical modelling workflows.

The deficiencies in our existing knowledge about the problem.

Since the 1980s, limited research has been conducted into developing 3D-printing workflows to suit the cellular make-up of the human body (which is organic in nature) (Bastawrous et al., 2018, p. 2809; Swetly et al., 2016, p. 145). Past bitmapbased 3D-printing studies have focused on the process of converting bioimaging medical data directly into 3D-printing files and physical models. However, past 
research does not address the replication of accurate anatomical colour and densities using a bitmap-based 3D-printing workflow.

The audiences that will benefit from a study of the problem.

This study was conducted by way of a research through design methodology. This methodology allowed for a thorough design exploration of bitmap-based 3Dprinting for medical modelling opportunities.

Bitmap-based medical modelling bares potential to be used as a replacement or adjunct to more traditional medical modelling workflows. This research may provide a general overview of novel high-end 3D-printing to the wider community, along with how to efficiently utilise the technology by emphasising the degree of detail that can be achieved by using a bitmap-based 3D-printing workflow to create highfidelity medical models.

This research aims to encourage collaboration between designers and medical professionals to push for the capabilities of new technologies - creating a future focussed dialogue. Subsequently, this may help to improve healthcare and save lives by democratising important information about novel technologies and bitmapbased 3D-printing workflows. In turn, this may improve communication between clinicians, families and communities.

The value of this may may be quantified through future studies. 


\title{
1. 4 RE S E AR C H D I R E T I O N
}

\author{
1. 4.1 \\ PURPOSE STATEMENT
}

The purpose of this creative design exploration is to investigate the possibilities of bitmap-based 3D-printing using biomedical imaging data to 3D-print patient-specific and high-fidelity medical models that mimic anatomical colour and density using the Stratasys J750 TM 3D-Printer, exposing the value of novel additive manufacturing methods for anatomical visualisation, communication and modelling.

\section{4 .2}

\section{RESEARCH Q UESTION}

What are the opportunities offered by high-resolution 3D-printing using a bitmap-based additive manufacturing workflow for highly realistic patientspecific medical modelling? 


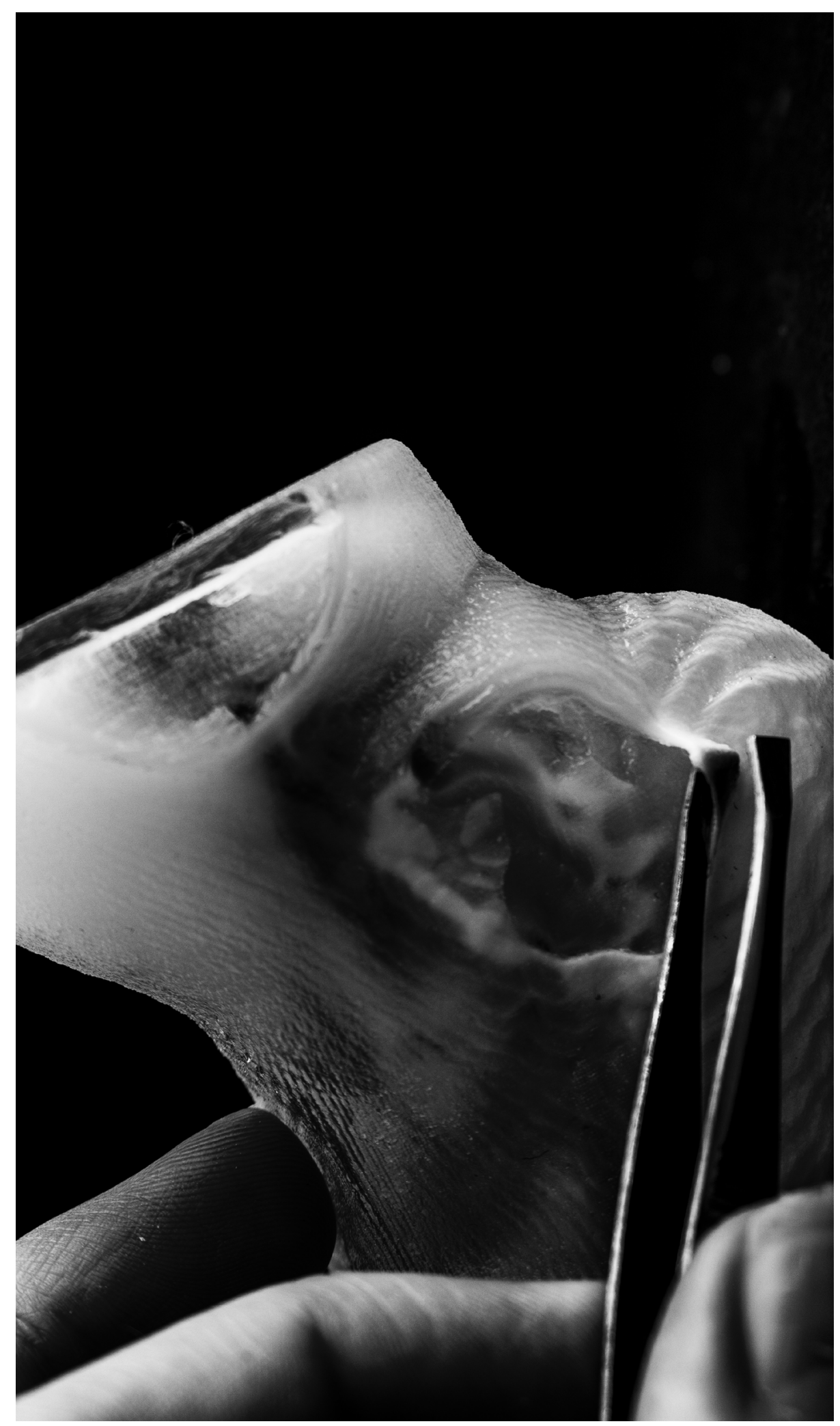

Figure 10. 3D-printed synthetic human skin. 

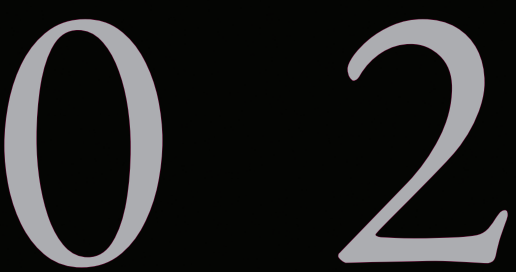

MET HOD OLO G Y

Left:

Figure 11. Taking notes from a book and reviewing literature. The book photographed in the image is Encyclopaedia Anatomica: A complete collection of anatomical waxes (p.58), by M. Düring, M. Poggesi, G. Didi-Huberman, 1999, Germany: Taschen. 
2.1 RESEARCH AIMS \& OBJECTIVES

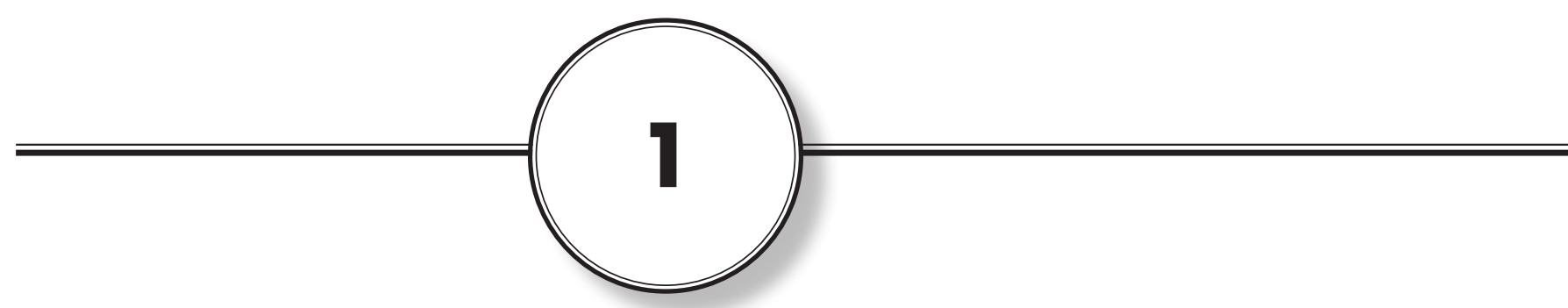

\section{A i m 1}

Situating the research within a body of knowledge of the discipline

Research medical modelling and bitmap-based additive manufacturing techniques to form the foundation of knowledge for future bitmap-based 3D-printing research.

O b j e c t ives

1a. Research the context of depicting human anatomy in order to understand the development of anatomical communication, creating an overview of important historical milestones and information (Historical Overview).

2b. Analyse literature regarding the current state of medical modelling and additive manufacturing technology, identifying the advantages and disadvantages of current practices, the value of 3D-printing medical models and the gaps in the current knowledge. (Literature Review)

2c. Develop initial design experiments to explore bitmap-based 3D-printing methods, establishing the foundation for a systematic enquiry (Parallel Prototyping). 


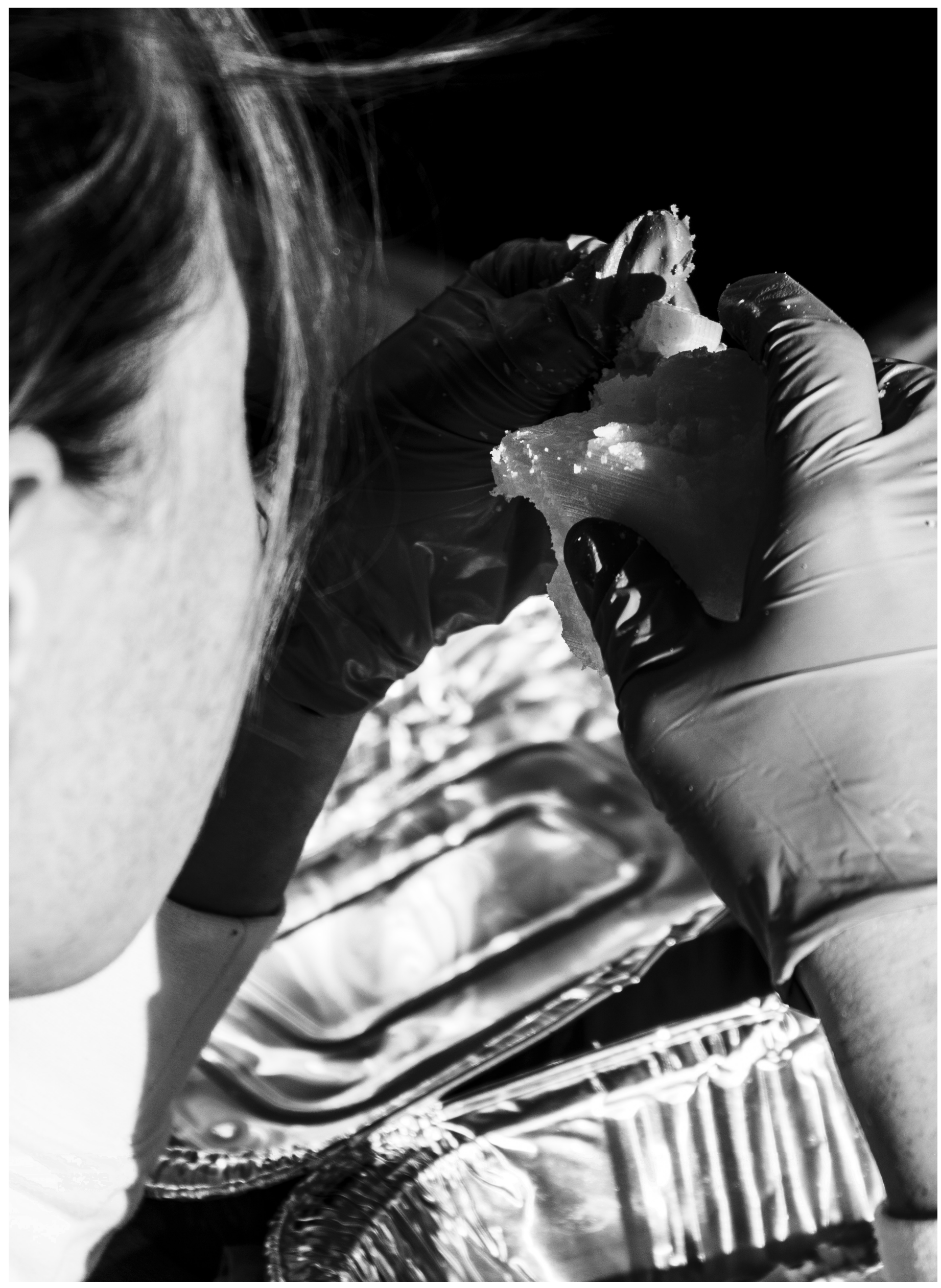

Figure 12. Removing support material. 
CHAPTER 2

\subsection{RESEARCH METHODOLO G IES}

Outlining a design methodology for design research environments is often challenging, and can be difficult to define based on the lack of a strict systematic enquiry. Many designers "struggle to situate their creative practice within a theoretical context” (Rodriguez Ramirez, 2017, p. 11). It is, therefore, important for design research to include focused creativity, whereby a designer has the freedom to explore a project within a set of criteria that guides their research. An iterative design process (referred to as "Practical Action Research") is moulded by design experimentation, critical reflection and design adaptation helps to form design outcomes. Engaging in a creative and iterative design process in this manner must follow a structure. However, the design process, equally, cannot be subjected to strict research restrictions that hinder creativity. The overarching aim of this chapter is to clearly outline a research design methodology and methods that will systematically guide this research portfolio without forfeiting the creative element of design research.

The overarching methodologies that this research followed were Research into Design, Research through Design and Research as Design. More specifically, this research portfolio followed a Research through Design methodology with a criteria-based approach outlined by Rodriguez Ramirez (2017). This research framework was initially outlined by Christopher Frayling in 1933 (Frayling, 1993) and has since been adapted by many design researchers such as Rodriguez Ramirez (2017). 


\section{RESEARCH INTO DESIG N}

involves situating the research within a body of existing knowledge. This research methodology includes investigating the traditional history, theory, criticism, as well as research into technical, material and structural aspects of the design field. Research into Design is the design activity which studies design including historical and theoretical research. It therefore helps to set a foundation for future design work based on past research (Frayling, 1993, p. 5).

\section{RESEARCH THROUGH DESIG N}

is the activity of creative exploration and reflection. Predominantly, Research through Design involves iterative models, material and colour tests, and feedback from experts. The iterative process was recorded and contextualised by communicating the design steps in detail through photography, text and physical prototypes (Frayling, 1993, p. 5). Research through Design was best suited to this research because the methodology is centred around the idea of studio-based, "hands-on" design conducted through practice-led research (Frayling, 1993, p. 5).

\section{DESIGN AS RESEARCH}

runs parallel to Research through Design and suggests that discoveries made through the material exploration of tangible models can constitute the production of new, innovative knowledge (Hanington \& Martin, 2012, p. 146). This research methodology was selected because the design process focusses on material exploration and design technique (rather than finding information through interviewing or "human-centred" design practices).

Throughout this research, these three methodologies worked in combination with each other, focussing on responding, adapting and critically reflecting on designs to reach an "appropriate" design solution (Hanington \& Martin, 2012, p. 146). It is important to note that design practice inherently involves risks, failures and successes. Therefore, the emphasis behind the methodologies selected is not about defending the designs - rather, the goal is to learn and adapt from "failures" to reach a valuable design conclusion. Accordingly, the end-design does not need to address all criteria to be treated as successful. 


$$
\ddot{\nabla}
$$




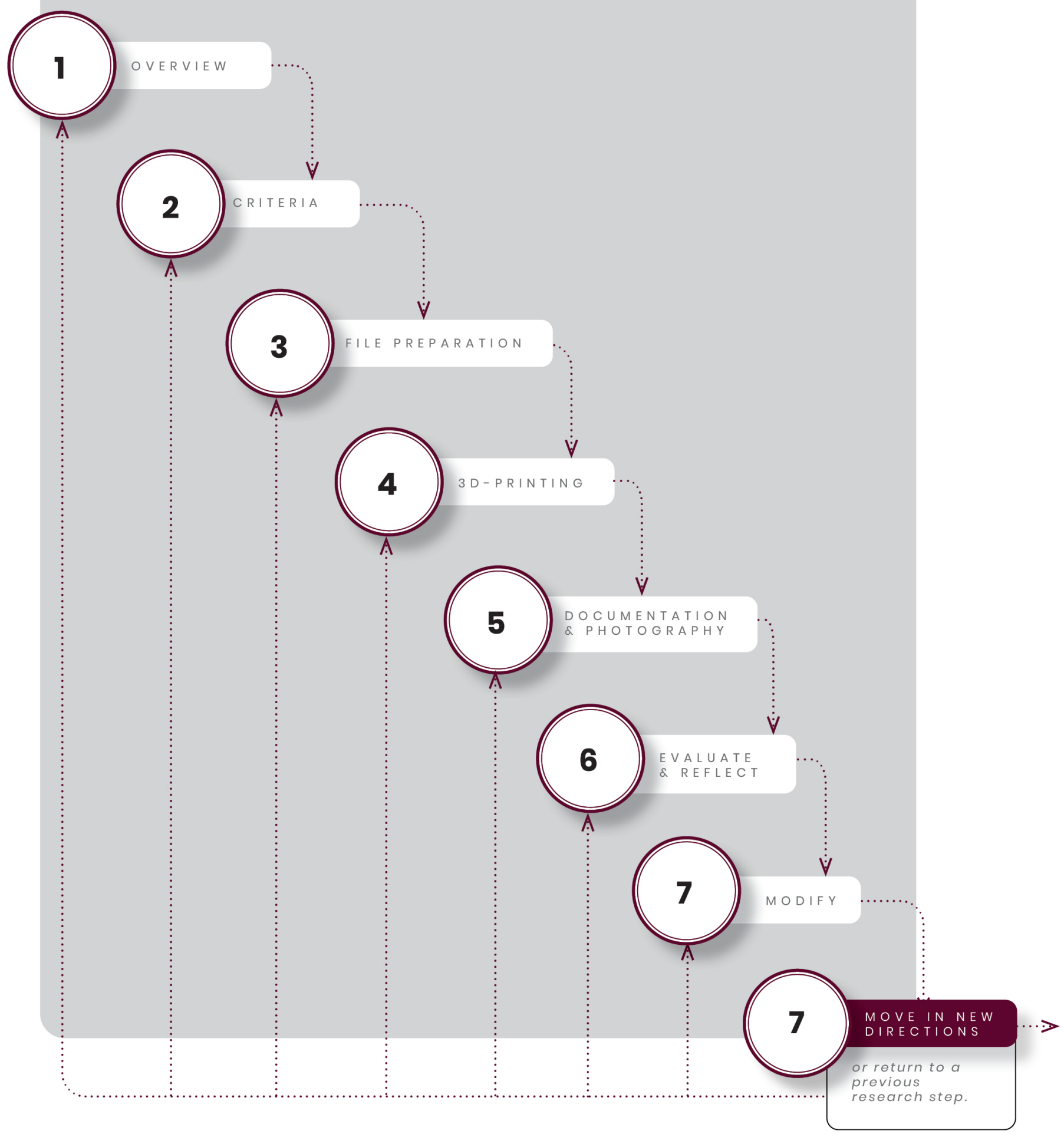

Figure 14. Practical action research cycle. 


\section{2.2 \\ Practical Action Research cycle}

(Crouch छ Pearce, 2012, p. 138; Frayling, 1993, p. 5)

The Results Chapter of this research followed a Practical Action Research Cycle. This cycle involved observing, reflecting, planning and acting with the end results being the development of new practices and knowledge. Using Design Criteria as a starting point, the iterative Action Research Cycle emphasised reviewing and revisiting designs to reach a design conclusion.

1. OVERVIEW

This step involved planning the research, developing criteria and identifying the main concern that could be solved through design.

2. CRITERIA

This stage involved reflecting on possible 'solutions' to the main design problem. This began by setting initial design criteria that guided each Case Study.

3. FILE PREPARATION

This research step involved producing physical 3D-printed models. 3D-printed models are created through different file preparation workflows that differ depending on the desired outcome.

4. $3 D-P R I N T I N G$

This research stage involved 3D-printing and cleaning the 3D-prints. Any adjustments to the model (dyeing, cutting, soaking, etc.) happens during this stage.
5. DOCUMENTATION \& PHOTOGRAPHY

This research stage was concerned with observing, tactile interaction and monitoring design outcomes by documenting the 3D-printed models through photography, film and notes.

6. EVALUATE AND REFLECT

This research step focussed on evaluating the designs by highlighting the disadvantages and advantages of the design technique. The design analysis helped to identify new design opportunities, including particular elements of the design process that had potential for readjustment and improvement. This stage was conducted through self-reflection, material observation, conversations with experts and analysis from Bernard Guy, the primary research supervisor.

7. MODIFY

Design modification during this research step was used to identify ways to move forward, adopting suggested ideas for the development of refined designs.

8. MOVE IN NEW DIRECTIONS OR RETURN TO A PREVIOUS RESEARCH STEP

This research step involved shifting the area of focus and forming a new set of criteria or returning to previous research steps and tweaking designs. 


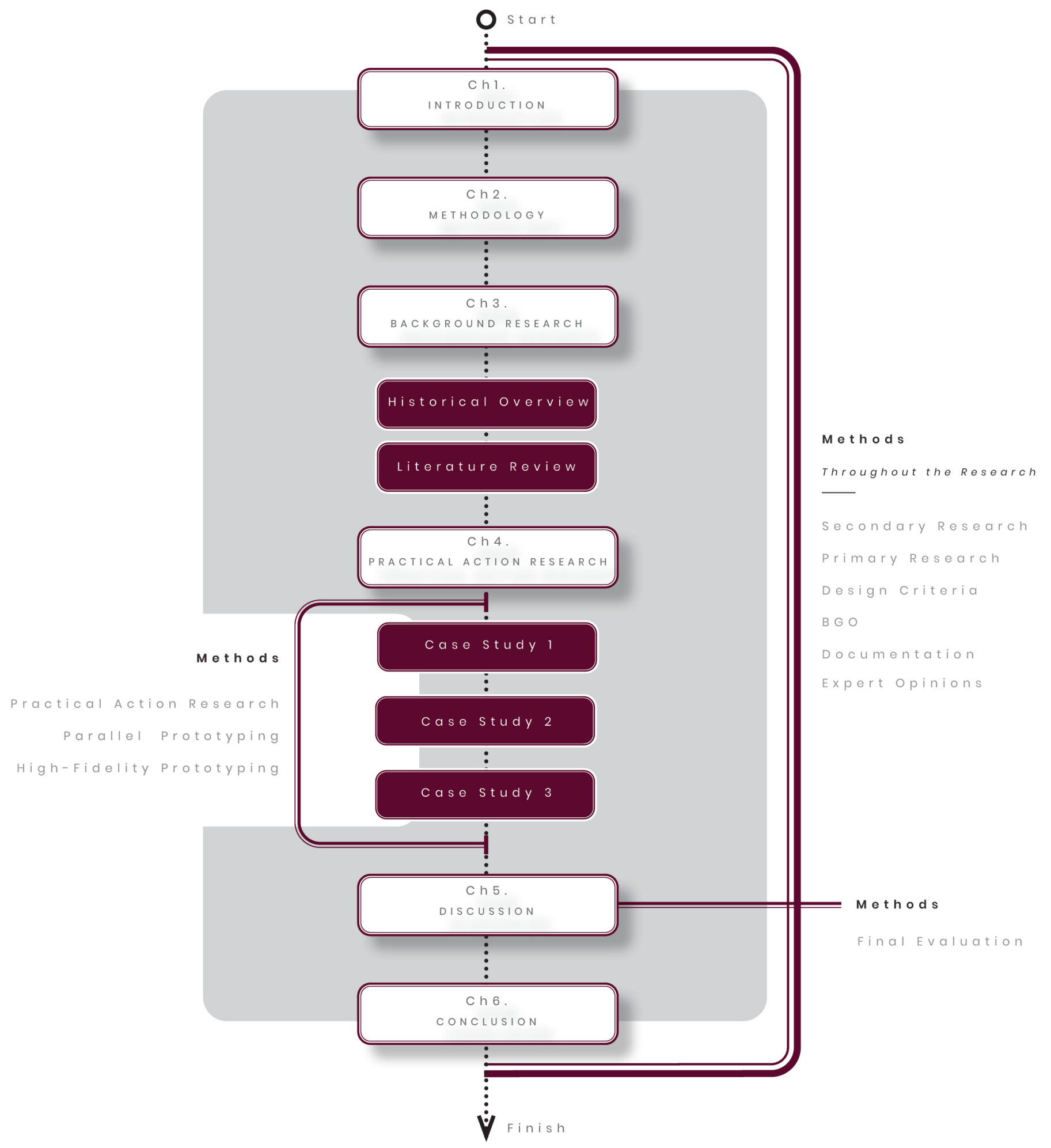

Figure 15. Outline of research methods. 


\subsection{RESEARCH METHODS}

\section{SECONDARY RESEARCH}

Information from existing data, including journal articles, books, reports and other similar documents.

PRIMARY RESEARCH

Information collected primarily as 3D-printed prototypes and subsequent analysis as well as information collected through current medical modelling company studies and discussions with experts.

DESIGN CRITERIA

A criteria-based research approach allows creative design practice to become a systematic form of enquiry (Rodriguez Ramirez, 2017, p. 12). Design criteria was outlined at the start of each Case Study, guiding and serving as a starting point for design experimentation, development and refinement (Rodriguez Ramirez, 2017, p. 13). Results developed during each Case Study were evaluated against the outlined criteria in the Discussion (ch.5).

BRAINSTORM GRAPHIC ORGANISERS ( $B G O$ )

A graphic representation of new knowledge that includes brainstorm webs, tree diagrams, flow diagrams to help with organising information and organizational thinking in 'networks' creating research frameworks and fluency of thinking. (Hanington \& Martin, 2012, p. 22).
DOCUMENTATION

The entire research process was documented to communicate, synthesize and record design results. This was achieved through photography, videography, material analysis and note taking (Hanington \& Martin, 2012, p. 146).

EXPERT OPINIONS

The MADE Dril stream has a current ethics approval for 'Gaining an Expert Opinion' from Industry (Application ID: 0000024294). Expert reviews and opinions were given by specialists in the areas explored. The specialists consulted with included (but were not limited to) biomedical engineers, the team at Stratasys, neurologists, anaesthetists and neonatal anatomy specialists. The project supervisor can account as an expert reviewer or opinion if the feedback is given objectively (Rodriguez Ramirez, 2017, p. 24). 
HISTORICAL OVERVIEW

An outline of the history behind understanding within the human body and how anatomical knowledge developed to where it is today. This was conducted by gathering historical sources and collating information (Frayling, 1993, p. 5).

LITERATURE REVIEW

A review of the relevant literature was conducted to gain knowledge of relevant disciplines. It was guided by the initial research question and project aims and objectives. The Literature Review focussed on the value of medical modelling and its associated problems as well as gaps in current research (Rodriguez Ramirez, 2017, p. 25).

The Literature Review captures the essence of previous research and aims to answer the following questions (Rodriguez Ramirez, 2017, p. 25).

- What do we know about the topic in the discipline?

- What is the debate?

- What future research has been suggested? 
CASE STUDIES

A case study research strategy was applied during the primary research element of this project presented in the Practical Action Research Chapter.

The purpose of including three research case studies was to highlight different medical modelling workflows, with a primary focus of achieving the research aims and objectives (specifically research aims 2a., 2b., and 2c.).

Each case study discussed in this research had a different set of criteria that were guided by a specific research objective. Throughout the research, the case studies are presented in chronological order based on the time of completion. This process involved selecting a specific situation with a corresponding medical dataset; once the dataset was selected, 3D-prints were produced based on the outlined criteria.

The Practical Action Research chapter is separated into three case studies:

1. Technique Exploration

Developing a bitmap-printing workflow with a focus on colour.

2. Design Discovery

Material exploration with a focus of mimicking attenuation (H.U) properties in bitmap-based 3Dprints.

3. Stratasys J750 тm Digital Anatomy ${ }^{\mathrm{T}}$ 3 D-Printer Study

Exploring novel medical modelling materials.
PARALLEL PROTOTYPING

Parallel Prototyping was used during Research Stage Two whilst conducting free design exploration (Crouch \& Pearce, 2012, p. 138; Frayling, 1993, p. 5). Parallel prototyping is the action of simultaneously exploring multiple design opportunities. Material exploration was conducted using parallel prototyping methods. The process included producing multiple 3D-printed design experiments at once and comparing the outcomes to refine the direction of research and the preferred bitmap-based 3D-printing technique.

FOCUSSED PROTOTYPING

High-Fidelity prototyping was used to develop and focus refined final 3D-printed models at later stages during the case study investigations (Hanington \& Martin, 2012, p. 138). The translation of hypothesised ideas into refined tangible objects helped to realise the research and provide a means for analysis against the outlined design criteria.

FINAL EVALUATION

A final evaluation helped to determine the disadvantages and advantages of the bitmap-based 3D-printed medical models. Avenues for further exploration were identified based on the information and new knowledge discovered throughout this research (Rodriguez Ramirez, 2017, p. 14). 
2.4.1

3 D - Printers

Table 1. THE STRATASYS J750 IM 3 D-PRINTER SPECIFICATIONS

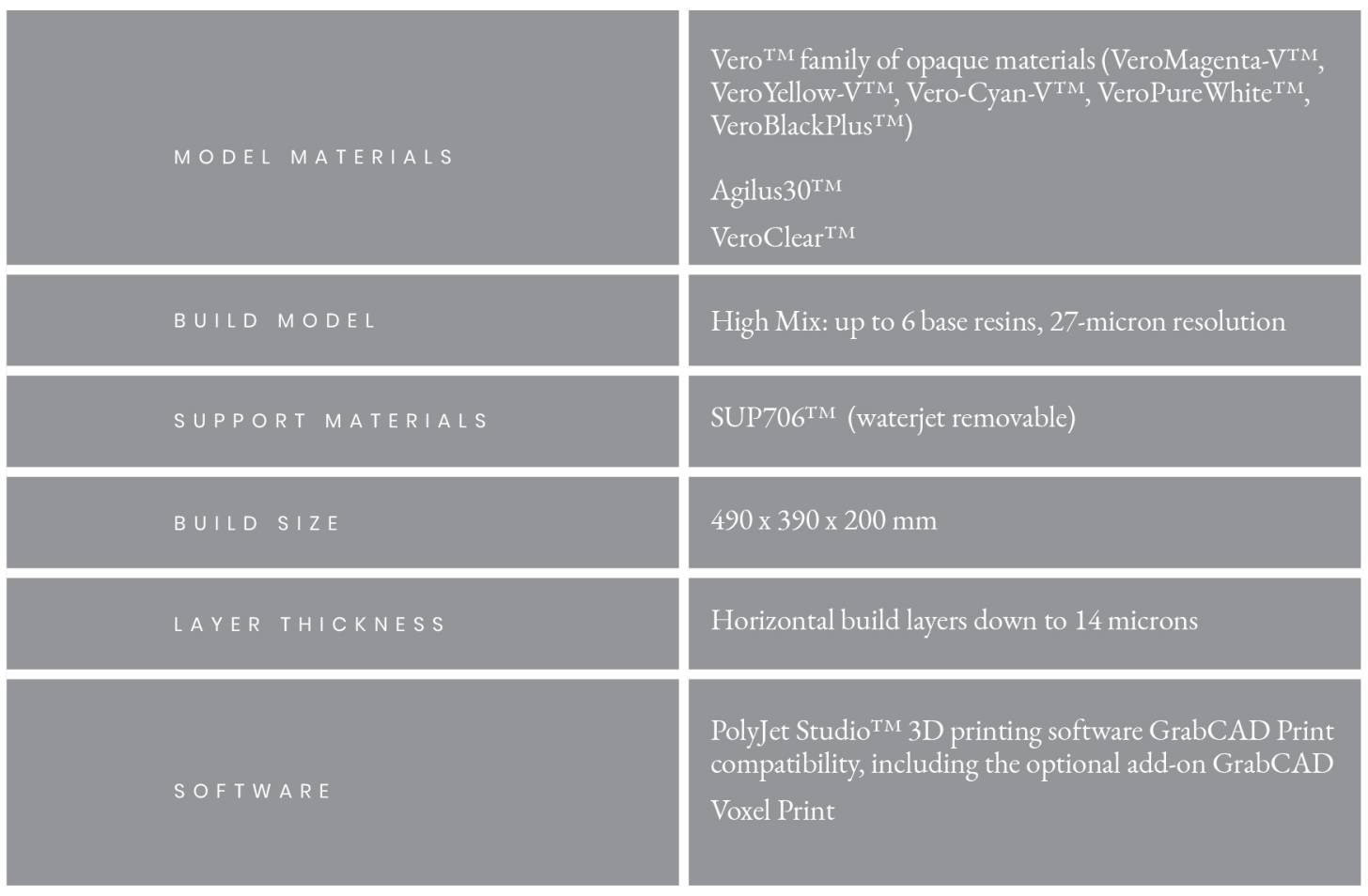




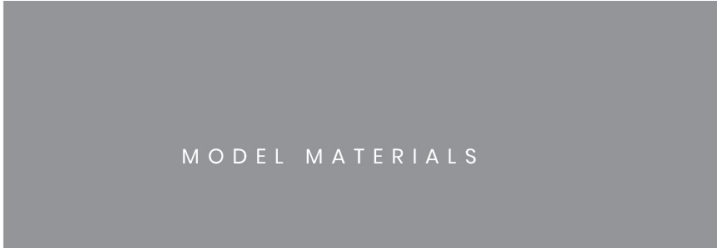

BUILD MODEL

SUPPORT MATERIALS

BUILD SIZE

LAYER THICKNESS

SOFTWARE
VeroYellow-VTM, VeroPureWhite ${ }^{\text {TM }}$, VeroMagenta-VTM Agilus30 $0^{\mathrm{TM}}$

TissueMatrix ${ }^{\mathrm{TM}}$

BoneMatrix ${ }^{\mathrm{TM}}$

J750TM materials also available

High Mix: up to 6 base resins, 27 -micron resolution

SUP705 ${ }^{\mathrm{TM}}$ (waterjet removable)

GelMatrix $^{\mathrm{TM}^{\mathrm{M}}}$ (waterjet removable)

$490 \times 390 \times 200 \mathrm{~mm}$

Horizontal build layers down to 14 microns

PolyJet Studio ${ }^{\text {TM }}$ 3D printing software GrabCAD Print compatibility, excluding the optional add-on GrabCAD

Voxel Print 
3 D SLICER

3D Slicer is an open source software platform for medical image informatics and image processing. 3D Slicer was used to segment parts of CT scans and to produce volumetric visualisations of medical data.

AUTODESK FUSION 360

Autodesk Fusion 360 is a 3D CA tool that was Autodesk Fusion 360 is a $3 \mathrm{D}$ CAD tool that was used to edit segmented meshes (STL files). Autodesk Fusion 360 was also used to produce any material swatch files.

R H I N O CEROS 3 D

Rhinoceros3D (dubbed 'Rhino') is a commercial 3D computer graphics and computer-aided design (CAD) application software. In this project, Rhino was used for the production of more complicated meshes of body parts.

A U TODESK MESHMIXER

Autodesk Meshmixer is a free $3 \mathrm{D}$ sculpting-based CAD programme created by Autodesk as part of their “123D” software line. The Meshmixer software was used in this project to repair any problems in the mesh as well as to slice and scale mesh files.

A UTODESK NETFABB

'repair script' on Autodesk Netfabb was utilised to fix any details in models to prepare them for 3D-printing. The Autodesk Netfabb 'Boolean' function allows users to cut a 3D part away from another 3D part.
FIJI - IMAGE J

ImageJ (also known as Fiji) is a Java based, open source image processing program, bundling a wide series of plugins that facilitate scientific image analysis. Fiji is the "batteries-included" distribution of Image J and was used to analyse medical data. The software was used to re-slice medical data image stacks.

A D OBE ILLUSTRATOR

Adobe Illustrator is graphic-driven software used primarily for creating vector graphics. Adobe Illustrator was used in this project for graphic design purposes including infographics.

ADOBE PHOTOSHOP

Adobe Photoshop is a raster graphics editor developed and published by Adobe Inc. Adobe Photoshop was used to automatically process bitmapbased 3D-Printing files.

GRABCAD PRIN T

GrabCAD Print is a programme that streamlines the 3D-printing workflow by setting up the printer tray, material colour and density selection specific to the Stratasys Polyjet 3D-printing system.

GRABCAD VOXEL PRINT UTILITY

The GrabCAD Voxel Print Utility is a software add-on for GrabCAD Print which widens the capabilities of the kind of parts you can print on a Stratasys $\mathrm{J} 750^{\mathrm{TM}} 3 \mathrm{D}$-Printer and is used to process bitmap files into 3D models and .GCVF files. 
2.4 .2

Data \& Ethics

CASE STUDY 1

Visible Human Project ${ }^{\circledR}$ Data Courtesy of the U.S. National Library of Medicine.

As of July 2019, the NLM Data License has been replaced by Terms and Conditions. No registration is required to access data available from NLM's FTP servers (The National Library of Medicines Visible Human Project, n.d.).

Images used were full color: The original cryosectional images at $0.33 \mathrm{~mm} \mathrm{XY}$ resolution, formatted in a lossless, directly readable .png format.

CASE STUDY 2

Ethics approval was granted through Dr. Ali Mirjalili at the (Senior Lecturer at the University of Auckland, New Zealand).

PROTOCOL REFERENCE \# 32679

Research protocol entitled, "Clinical anatomy of the infant: Correlation with clinical landmarks using imaging, digitization and 3D modelling”.

ETHICS APPROVAL

Original Approval Date: March 1, 2016

CASE STUDY 3

Same as Case Study Two. 


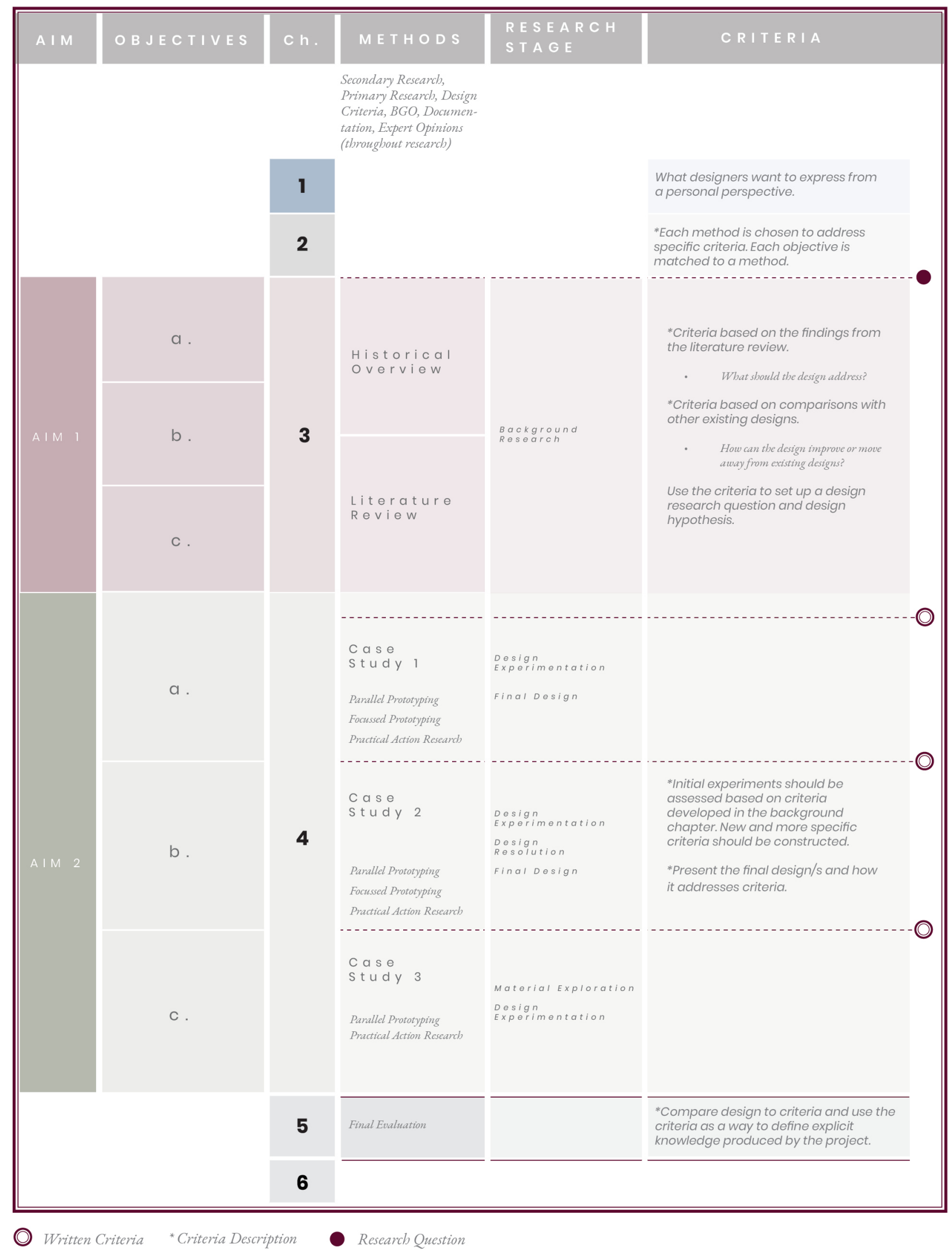

Figure 16. Methodology chapter summary. 

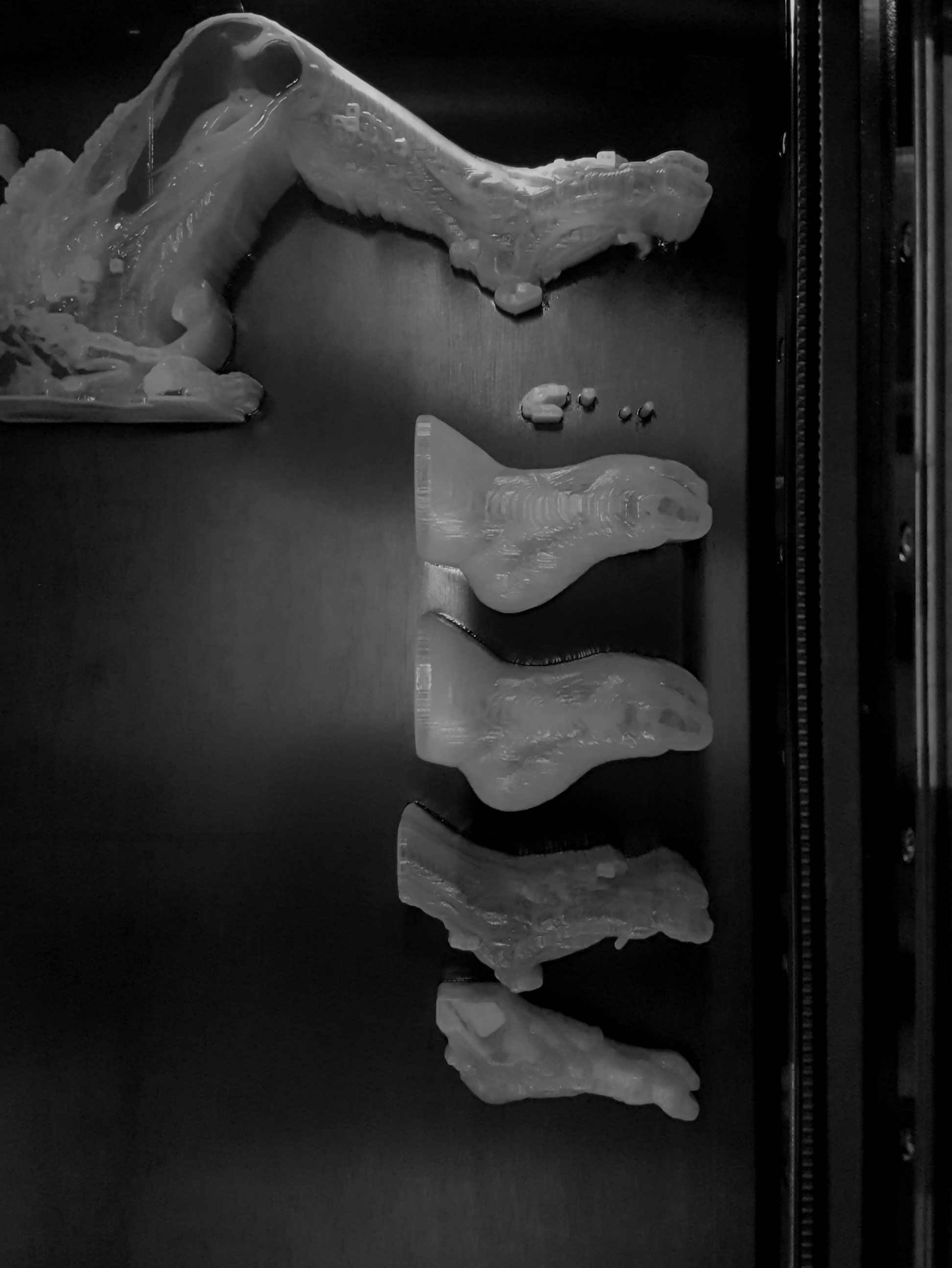

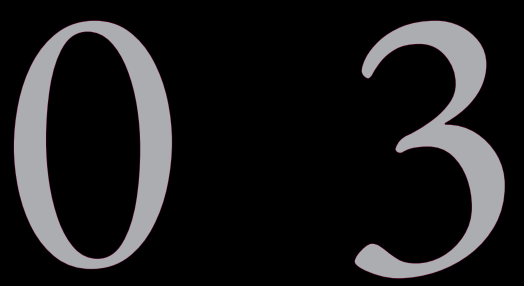

BACKGROUND RESEARCH

\section{Left:}

Figure 17. The Stratasys J750 ${ }^{\mathrm{TM}}$ 3D-Printer 3D-printing neonatal feet and a 1:1 scale neonatal leg ( $75 \%$ completed). 
CHAPTER 3

\subsection{H IS TORICAL O VER V IE W}

The History of Understanding Human Anatomy

Researchers from many fields have laid the foundation for contemporary anatomical knowledge. Scientists, physicians, artists, engineers, criminals, and martyrs were all instrumental in the progression of anatomical imaging - from ancient Egyptian papyri to the use of magnetic resonance imaging (MRI) (Owen, 2016).

The controversies associated with the study of human anatomy must also be acknowledged. Medical history is, tragically, riddled with many discomforting insights and atrocities, along with the exploitation of minorities and the vulnerable (Ghosh, 2015).

Figure 19. provides a brief outline of the historical development of understanding human anatomy. It seeks to illustrate that reproducing human anatomy at a high-fidelity has been central to medical progression.

Left:

Figure 18. Dr. Nicolaes Tulp demonstrating an educational dissection session explaining the physiology of the arm and exposing anatomy that lies beneath the skin. From The Anatomy Lesson of Dr. Nicolaes Tulp, by R. V., Rijn, 1632 CE, reproduced from the public domain. 


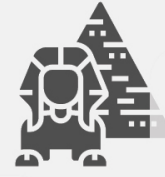

ANCIENT EGYPT

The first transcribed information about anatomy dates back to approximately 3000 B.C. in ancient Egypt (Loukas et al., 2011)

\section{0}

Historians attribute the wealth of ancient Egyptian anatomical knowledge to the role of the ancient Egyptian embalmer and physician (Huotilainen et al., 2014).

Kahun Papyrus (1825 B.C.), the Edwin Smith Papyrus (1700 B.C.) and the Ebers Papyrus (3000 B.C.)
HELLENISTIC PERIOD

During the Hellenistic period (323 B.C. - 31 B.C.) in the Ptolemaic Kingdom in Alexandria, the centre of research was the Alexandrian Library and Museum, a place rich in physiological and anatomical investigation (Loriaux, 2016). Here, Herophis and Erastatu valued contributions to anatomical sciences by conducting the first legal human dissections, or vivisections, on convicted criminals (Ghosh, 2015)

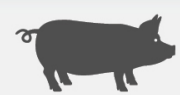

For more than 1000 years, physicians followed Greek physician living in Rome named Claudius Galen pen primates and swne (Lorian, 2016). However, he incorrectly extrapolated many of his findings to humans which misled physicians for many years.

A resurgence of medical explorati 13th and 14th centuries in Europe. the Middle Ages ( 476 A.D. - 1453 nd science, including anatomical st

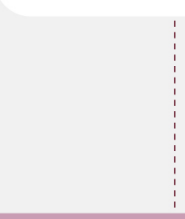

$3000 \mathrm{BC}$ o

\section{CLASSICAL GREECE}

IIII

The inception of Western medicine is said to have occurred in classical Greece (5th and 4 th centuries B.C.) (Loriaux, 2016).
Hippocrates, known as "the father of Western medicine" and one of the most celebrated physicians of antiquity, first recognised disease entities and that recoger disese (Loriaux, 2016)
MEDIEVAL AGE

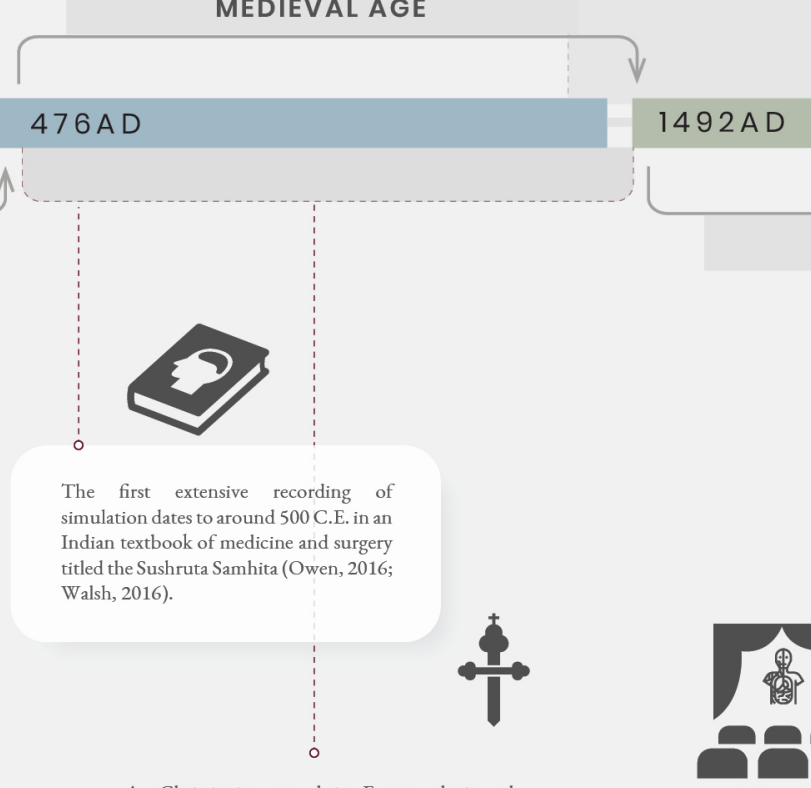

As Christianity spread in Europe during the Middle Ages ( 476 A.D. - 1453 A.D.), human dissection was viewed as blasphemous and was banned (Ghosh, 2015, p. 154). 
on commenced during the new way of thinking after .D.) manifested itself in art udies (Ghosh, 2015). ing the Renaissance professor in surgery Of Padua from 1537 through detailed sionalised anatomy sionalised anatomy
in his texts (Owen,

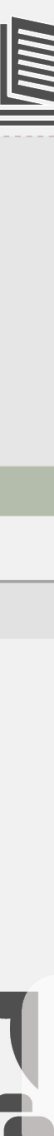
was inaugurated in 1595 by Girolamo Fabrici D'Acquapendente, also known as Fabricius, who held the chair of anatomy at Padua for about 50 years (Owen, 2016)

\section{iîi} í

Medical education in the 17 th and 18 th centuries included the use of wax models of anatomical figures. This form of wax modelling intertwined with religion and magic" (Owen, 2016). reflected "a time when science was still

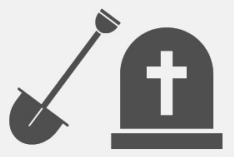

Sacrilegious grave robberies led to the 1828 case of Burke and Hare, who confessed to murdering 16 people to meet this demand (Ghosh, 2015).
At the end of the 19th century, the first stereoscopy atlas of medicine was produced by Albert Ludwig Sigmund Neisser, which meant that people could see diseases in three-dimensions (Owen, 2016).

The induction of the infamous Murder Act of 1752 made the dissection of criminal corpses compulsory (King, 2017). However, supply still did not meet demand. Anatomists acquired bodies illegally through grave roberies, the act of digging up graves and using bodies for dissection (Ghosh, 2015)
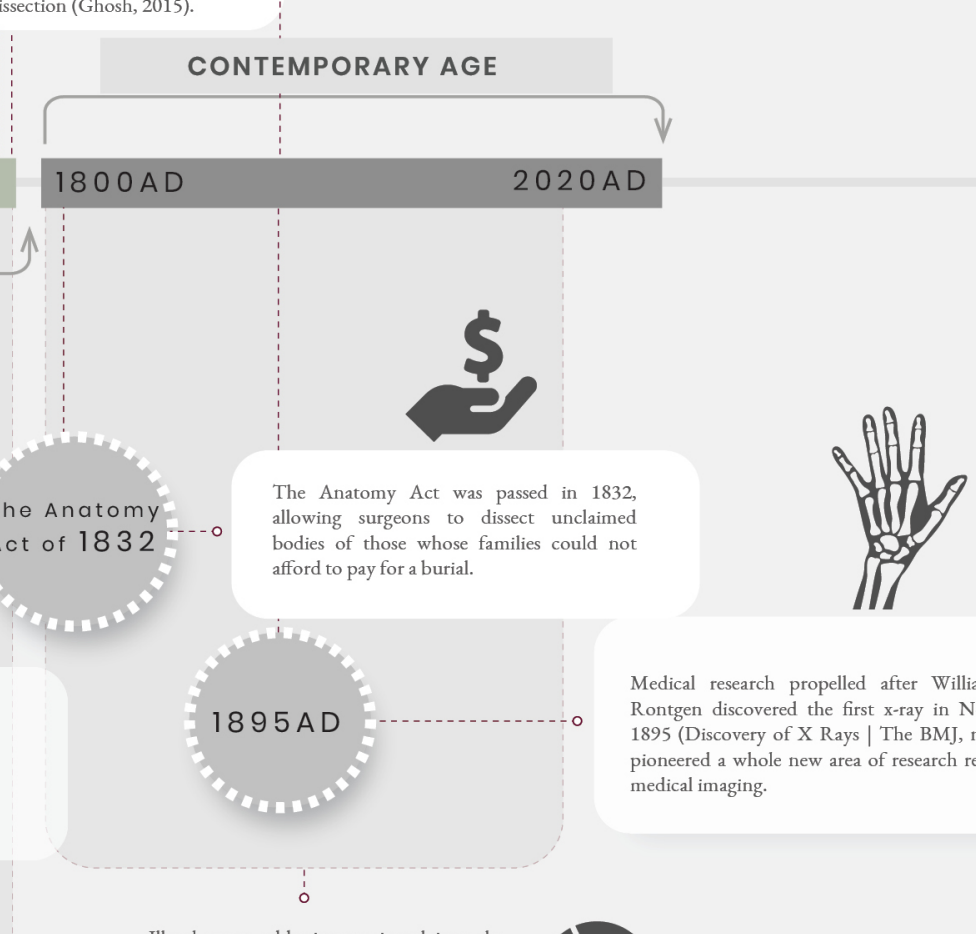
medical imaging.

Medical research propelled after William Conrad Ronten discoved Rontgen discovered the first $x$-ray in November of 1895 (Discovery of X Rays | The BMJ, n.d.). X-rays pioneered a whole new area of research referred to as

Figure 19. The history of understanding human anatomy (western history). 
iew of a fetus side the uterus arstellung eines ten in der Cebă utter etus dans l'utésouvert

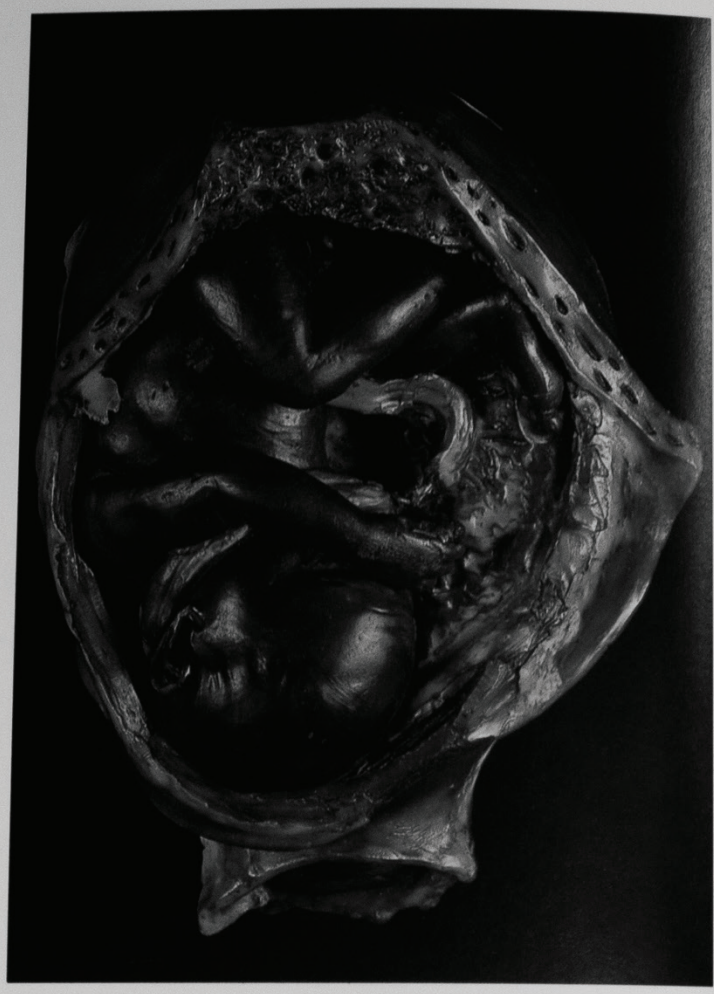

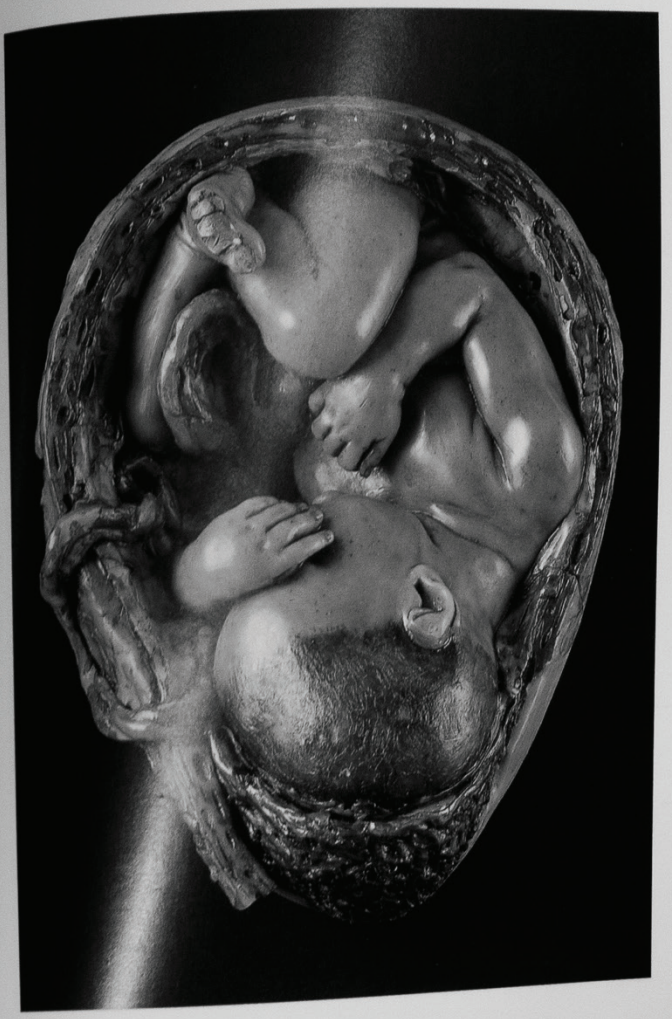

View of a fetus inside the uterus

Darstellung eines

Fôten in der Cebar

mutter

Foetus dans I'uté-

rus ouvert 
BACKGROUND RESEARCH

\section{2 L ITERAT URE RE V IE W}

The Literature Review will outline:

- Basic biomedical imaging principles.

- The value of physical modelling in comparison to virtual models.

- General 3D-printing.

- The value of physical medical modelling using high-resolution 3D-printing workflows.

Left:

Figure 20. Reading a book about anatomy. The book photographed in the image is from Encyclopaedia Anatomica: A complete collection of anatomical waxes (p. 674-675), by M. Düring, M. Poggesi, G. DidiHuberman, 1999, Germany: Taschen. 


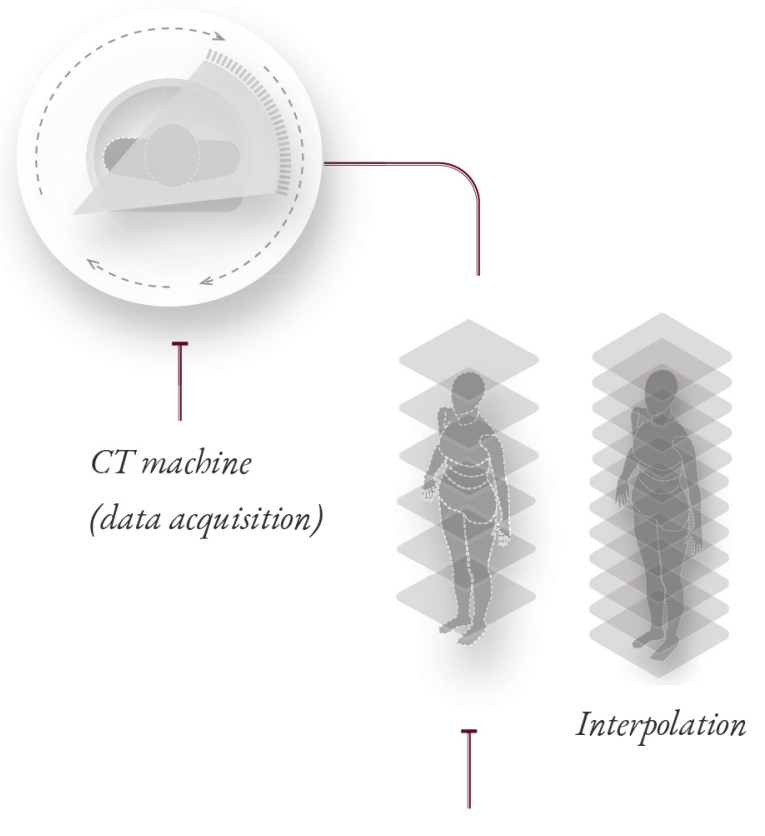

Cross-sectional CT images through the

buman body
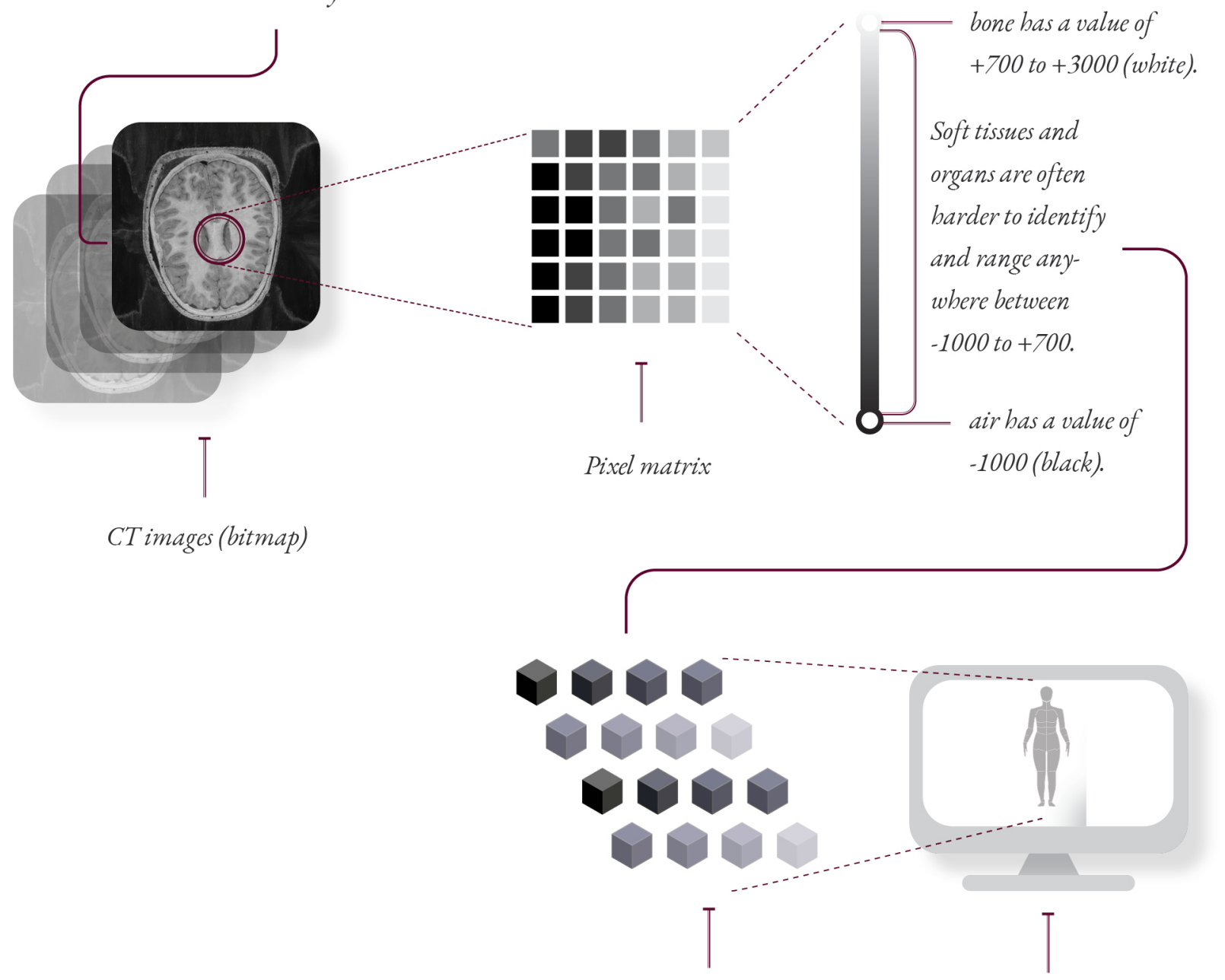

Volumetric pixel

Voxel representation of the buman (voxel or cuboid) body (virtual reconstruction)

Figure 21. CT imaging process (from data acquisition to virtual reconstruction). 


\section{2.1}

\section{Biomedical I maging}

Since the 1980s, visualising human anatomy through multiplanar reformations, maximum intensity projections, volume renderings, and cinematic renderings for the purpose of understanding physiology has been an area of extensive exploration (Fellner, 2016, p. 170). Cross-sectional images through the human body can generate $3 \mathrm{D}$ reconstructions of anatomy. Specialists capture anatomical images using scanning modalities, including computed tomography (CT) and magnetic resonance imaging (MRI) (Bibb et al., 2015, p. 7). The appropriate scanning modality is selected based on the objective of the scan, the area of anatomy and the desired image quality.

COMPUTED TOMOGRAPHY ( C T )

CT imaging entered the world of medicine due to the advancement of computers and allowed the capture of multiple tomographic images (slices) of the body (Bradley, 2008, p. 351).

\section{Measuring Density}

CT works by measuring the amount of energy absorbed by the body as an X-ray tube rotates around a patient. Various detectors pick up the X-rays that are not absorbed, reflected, or refracted (Bibb et al., 2015, p. 8; Bradley, 2008, p. 351). When CT data is captured, the resulting images are divided into greyscale pixel matrixes (Bibb et al., 2015, p. 9). Each pixel is a shade of grey that relates to the density of tissue at that location and the density is quantified using the Hounsfield unit (H.U.) scale (Bibb et al., 2015, p. 8).

In a CT image, air has a value of -1000 (black on the greyscale) and bone has a value of +700 to +3000 (white on the greyscale) (Bibb et al., 2015, p. 8). Soft tissues and organs are often harder to identify and range anywhere between -1000 to +700 (appear in different shades of grey) (Bradley, 2008, p. 8). One pixel can only have one threshold or H.U. value, and therefore its value is determined based on which tissue type fills the majority of the pixel (Bibb et al., 2015, pp. 9-10). 


\section{The Volumetric Pixel}

If the distance between axial images (slice thickness) is known, software can allow for interpolation between cross-sectional tomographic images to create volume reconstructions with smooth surfaces (McGurk et al., 1997, p. 170). Voxels are formed by interpolating one image to the next, creating a voxel representation of the human body (Bibb et al., 2015, p. 9). These voxel datasets can then be resliced on the different axes to generate cross-sectional views of the data (Bibb et al., 2015, p. 9).

MAGNETIC RESONANCE IMAGING (MRI)

MRI works similarly to CT imaging. However, instead of measuring density, MRI measures the amount of water in the body. MRI scanning targets the hydrogen nuclei present in water molecules and; therefore, areas of the body with less water content show up lighter, and areas with more water show up darker (Bibb et al., 2015, p. 21). For example, air and dense bone will appear black, whereas fat will appear white (Bibb et al., 2015, p. 21). MRI images also have significantly larger pixel sizes than CT images.

Chung and Park (2007) argue that virtual reconstructions of anatomy with virtual reality environments could compensate for the limited availability of cadavers due to time, place and cultural limitations of cadaver dissection. Pommert et al. (2001) also suggest that computer-based representations of biomedical imaging datasets are superior to traditional methods of medical communication where anatomy is presented in books and atlases.

Wu et al. (2012) challenged this idea by stating that thin-sectional images of human cadavers (cryosectioning) can be used to construct realistic 3D models of anatomy without any loss of information and in true colour (which is beyond the reconstruction capabilities using greyscale biomedical imaging datasets). Chung \& Park (2019) validate the proposed value of cryosectioning through their highly realistic virtual reconstruction created by using the Visible Korean Human (VKH) dataset. However, cryosectioning cannot be conducted on live patients. Biomedical imaging modalities, on the other hand, can produce crosssectional images of live patients, allowing the reconstruction of patient-specific anatomy in living patients. 


\subsection{2 \\ Physical Modelling in Medicine}

VIRTUAL REALITY VS. PHYSICAL MODELLING

Physical or virtual medical modelling is almost always used to provide a complementary educational experience to cadaveric dissection. Whether medical models are created for surgical simulation, pre-operative planning, patientsurgeon communication, product testing, didactic instruction, prosthetics or biomechanical research purposes, the end goal is almost always to improve and accelerate the quality of medical treatment and experiences (Palter \& Grantcharov, 2010, p. 1195).

Many academics and clinicians question whether there is value in physical modelling considering Virtual Reality (V.R.) environments can also incorporate the use of realistic haptic feedback (Lioufas et al., 2016; Panait et al., 2009). Physical medical modelling could be made redundant once V.R. technologies develop, presenting a justified concern about the future of physical modelling. V.R. models can now incorporate tactile and kinaesthetic receptors to allow surgeons to feel force feedback while using surgical instruments (Lioufas et al., 2016; Panait et al., 2009). A medical student or professional that immerses themselves in this kind of sophisticated V.R. environment, can see a rendered visualisation of bone and tissue and can feel virtual contact forces while interacting with different anatomy (Hochman et al., 2015, p. 264).

However, a drawback of most haptic V.R. models is that the "stiffness" of the bone and tissue is not as realistic as most physical models (Hochman et al., 2015, p. 263). Additionally, tools such as robotic drills cannot be tested on V.R. models meaning medical procedures cannot be conducted in their entirety (Hochman et al., 2015, p. 264). Authors have criticised current V.R. models because of the high initial cost of system acquisition, limited force feedback with the use of surgical instruments, and the lack of realism in graphics (Bader et al., 2018; Breimer et al., 2017; Palter \& Grantcharov, 2010). 
The available literature concludes that the technology chosen for anatomical modelling should be selected based on specific learning objectives and training goals (Breimer et al., 2017, p. 93). For example, evidence shows that high-fidelity physical simulator models are more useful for practising complex procedures, whereas V.R. simulators work well for teaching fundamental technical skills (Palter \& Grantcharov, 2010, p. 1194). A consensus gathered from literature was that "depending in the perceived educational requirements, it is possible that one system [V.R.] or the other [3D-printing] may provide the most useful platform" (Hochman et al., 2015, p. 264).

Breimer et al. (2017) compared physical and V.R. neurological simulators. Their study concluded that a physical simulator is a better tool for applications such as familiarising trainees with different instruments and techniques, helping with teamwork, communication and different bleeding scenarios. The V.R. model was better suited for learning anatomy in three-dimensions. The results of the study indicated that $50 \%$ of participants stated that the physical model was superior, $12 \%$ of participants indicated that the V.R. model was superior, $19 \%$ of participants indicated that the V.R. model was better for learning anatomy and the physical model was better for learning instrument handling techniques and the final $19 \%$ of participants were undecided.

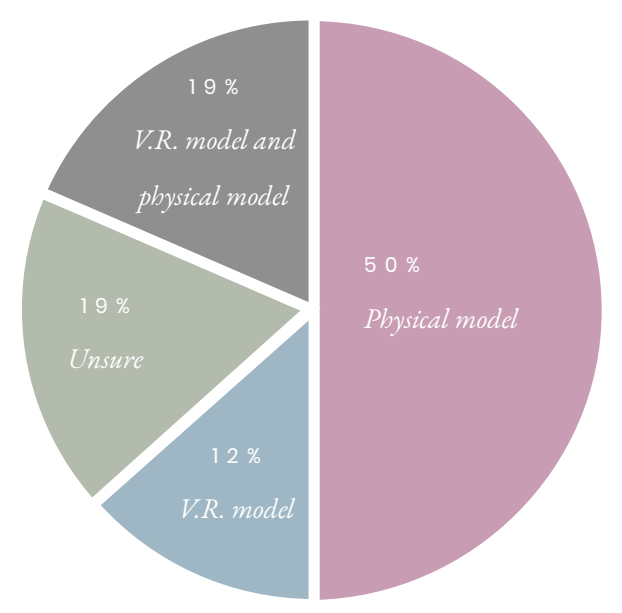

Figure 22. Virtual Reality vs. Physical Model

(Neurosurgical Resident Preference) 


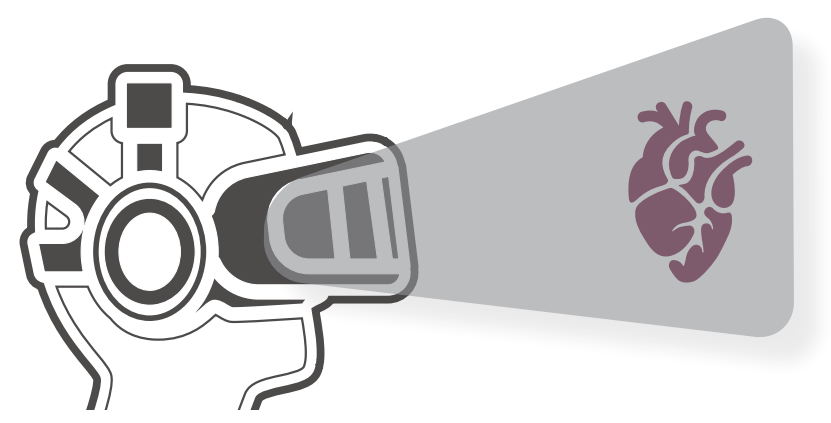

Figure 23. Person experiencing V.R. environment.

Scerbo et al. (2006) compared the effectiveness of a V.R. simulator in comparison to a traditional plastic limb for training phlebotomy (drawing blood). Improper performance of this procedure can lead to some complications, including injuries and inaccurate test results (Scerbo et al., 2006). In their study, medical students were trained under one of the two methods.

Overall, results showed that students performed better on a physical model rather than in a V.R. environment. Authors concluded that ultimately the central factor of success for a simulation device is the realism and fidelity of the training task. Procedures such as cutting organs, checking a patient's pulse and using medical instruments sometimes rely solely on a medical professional's sense of touch. Therefore, having realistic haptic feedback is imperative to a useful simulation device. Scerbo et al. (2006) concluded that currently there is more evidence to suggest that a physical simulator is a more effective method of teaching.

There are a limited amount of studies that have quantified the effectiveness of physical modelling in comparison to V.R. models for medical simulation and educational purposes. Existing studies conclude that, overall, a higher percentage of surgical trainees and surgeons preferred learning on a physical model. Currently, literature shows that both modalities have strengths and weaknesses and that establishing the long-term effectiveness and superior modelling method of V.R. in comparison to physical modelling will take time and an extensive body of research (Breimer et al., 2017; Cromeens et al., 2017; Hochman et al., 2015).

3D-printed medical models are useful tools for resident education and pre-operative surgical rehearsal when compared to a V.R. model with haptic feedback. In a study conducted by Hochman et al. (2015), a haptic voxel-based virtual model and a physical 3D-printed temporal bone model were developed from CT data. Resident surgeons were asked to rate their experience on both simulators based on a range of different parameters. Most resident surgeons agreed that both models were productive adjuncts to traditional educational resources and could help in improving surgical skill acquisition. However, the 3D-printed model was perceived to be more productive and was the preferred training tool across most domains due to the lack of realism of the haptic feedback of the virtual model. 
Three-dimensional (3D) printing technologies offer great opportunities for physical anatomical modelling. 3D-printing is a manufacturing technology that can create physical forms from computer-aided design (CAD) models. Originating in the early 1980 s, 3D-printing was first introduced to produce prototypes in a quick and automated manner and found great success in areas such as aerospace, automotive, military and architecture (Bastawrous et al., 2018, p. 2809; Swetly et al., 2016, p. 145). Unlike traditional subtractive manufacturing methods, 3D-printing works by depositing material, layer-by-layer, to create a physical model. Machines can now efficiently produce objects with less material waste and associated costs (Watson \& Taminger, 2018, p. 1317).

The seven main 3D-printing categories include Binder-jetting, Vat photopolymerisation, Material Extrusion, Material jetting, Powder bed Fusion, Directed Energy Deposition and Sheet Lamination (Bastawrous et al., 2018, p. 2811). Each AM technology has its advantages and disadvantages and therefore deciding on which 3D-printing technique to use is dependent on cost, production time, material qualities, colour, intended use and detail requirements (Bastawrous et al., 2018; Swetly et al., 2016).

As the efficiency of 3D-printing manufacturing processes has improved, the 3D-printing market has grown drastically (Swetly et al., 2016, p. 145). The ambit of 3D-printing has expanded to include many different fabrication methods and $3 \mathrm{D}$-printer types, ranging from relatively cheap to highly sophisticated machines. 3D-printing can produce complex geometries, internal structures and models in a range of different materials. AM is also now used for the end-production of products - rather than prototyping, which accounts for a "growing portion of the total revenue of the global AM market" (Doubrovski et al., 2015, p. 4). The use of 3D-printing has a growing importance in the medical industry - from 3D-printing Personal Protection Equipment to anatomical models of patient-specific anatomy (Stratasys Responds to COVID-19 Pandemic, 2020). 


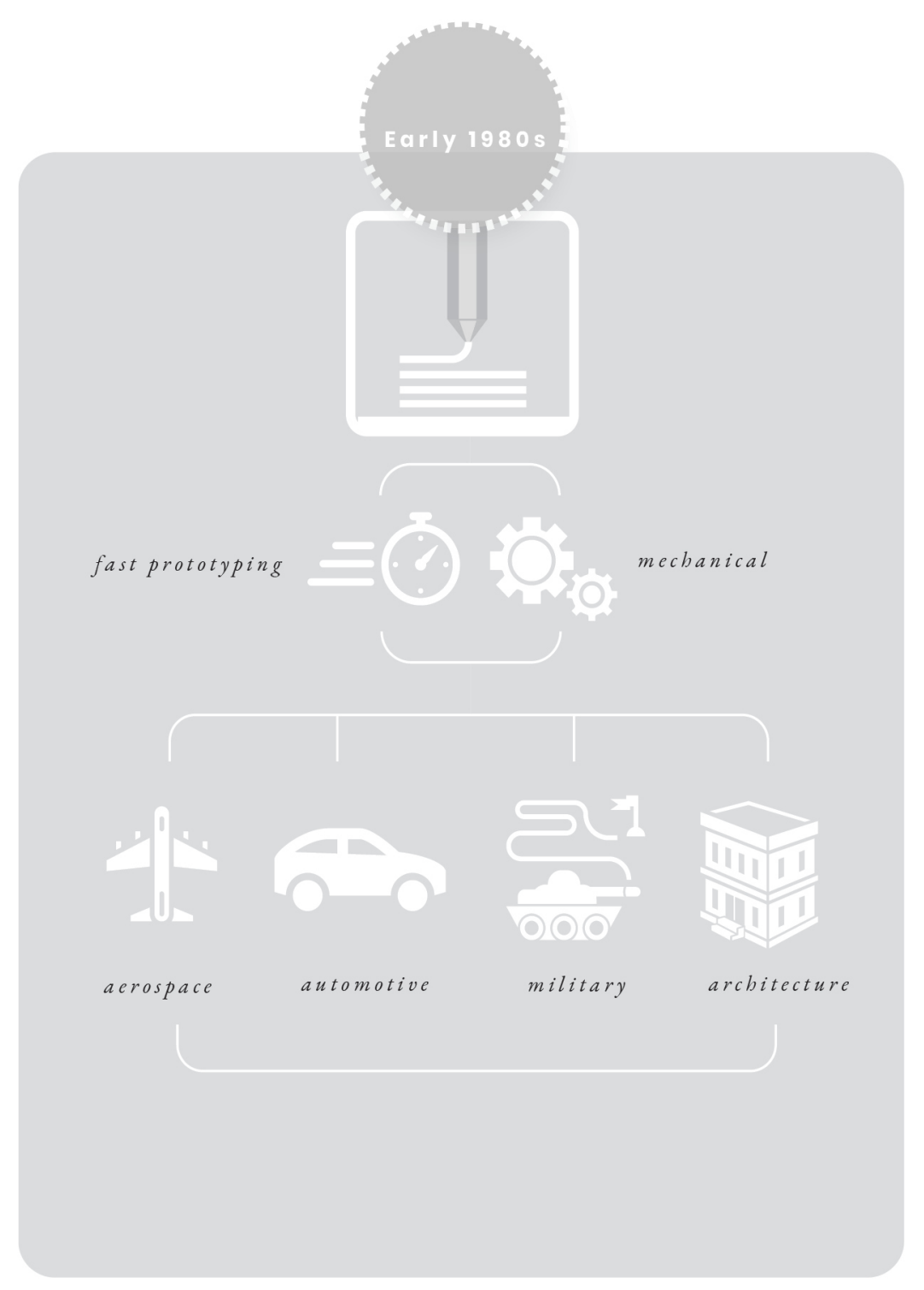

Figure 24. Origins of 3D-printing. 
MEDICAL MODELLING

Bader et al. (2018), Hosny et al. (2018) and Yang et al. (2018) suggest using medical modelling to produce physical anatomical models allowing medical physicians, students and patients to interact with tangible patient-specific medical data. AM methods can build medical models that are impossible to make through other subtractive manufacturing processes such as silicone moulding. The hierarchical, functional and material complexities that can be attained through AM produce an unparalleled level of detail in physical objects that may begin to emulate the fidelities of human anatomy to the highest degree of realism (Sossou et al., 2019).

Usually, 3D-printed anatomical models are produced from patient-specific biomedical imaging data through a process called segmentation and the subsequent isosurface extraction. This method is known as "medical modelling" (Bibb et al., 2015, p. 1). Medical modelling can replicate exact patient-specific anatomical geometries quickly and in large quantities. There is a plethora of evidence to suggest that medical modelling is beneficial for the study of human anatomy, patient-surgeon communication, pre-operative planning, surgical simulation and medical research (Rengier et al., 2010; Young et al., 2019).

An example of useful medical modelling has presented itself recently as a response to the impact and effects of the COVID-19 pandemic. This pandemic has placed an immense amount of pressure on healthcare institutions. AM has provided some relief in many areas. Fusetec, an Australian-based medical modelling company, has responded to the COVIS-19 pandemic by providing high-fidelity models of human nasal sinuses (FusetecSinus Trainer) to hospitals around Australia. The FusetecSinus Trainer has been distributed to hospitals to help ensure that front-line healthcare workers are appropriately trained in nasal swab collection techniques to test individuals properly, minimising the transmission of COVID-19 (Fusetec 3D Headquarters, 2020). 
Most 3D-printed anatomical models currently produced are created following a workflow involving image thresholding and isosurface extraction techniques, resulting in the aforementioned surface meshes (STL files) (Hosny et al., 2018; Huotilainen et al., 2014). This process is commonly referred to as segmentation. Research is currently being conducted into adapting traditional meshbased 3D-printing workflows to processes that are better suited to mimicking the fidelities of human anatomy, which is organic and cell-based in structure (Bader et al., 2018; Doubrovski et al., 2015; Hosny et al., 2018; Stute et al., 2018).

Current CAD programmes accommodate traditional segmentation manufacturing workflows. For example, Materialise Mimics is an image-processing tool that coverts biomedical imaging data into traditional mesh-based models ready for 3D-printing (Materialise 3D Printing Innovators, 2020). Currently, Materialise Mimics have a significant influence over the segmentation and medical modelling industry. Interestingly, however, literature describes medical modelling using traditional mesh-based workflows to be time-consuming, resulting in anatomically inaccurate 3D-prints caused by data loss, segmentation artefacts and multiple post-processing steps using different software packages (Bader et al., 2018; Bibb \& Winder, 2010; Hosny et al., 2018; Huotilainen et al., 2014).

Literature suggests that current workflows are limited, as shape specification is inherently linked with one colour and density - limiting the production of material gradients (Bader et al., 2018; Doubrovski et al., 2015; Stute et al., 2018). Mesh-based 3D-printing methods are inapt for 3D-printing complex material topologies (such as human tissue) and that production methods currently overlook the design potential of heterogeneous material distributions (Bader et al., 2016). 


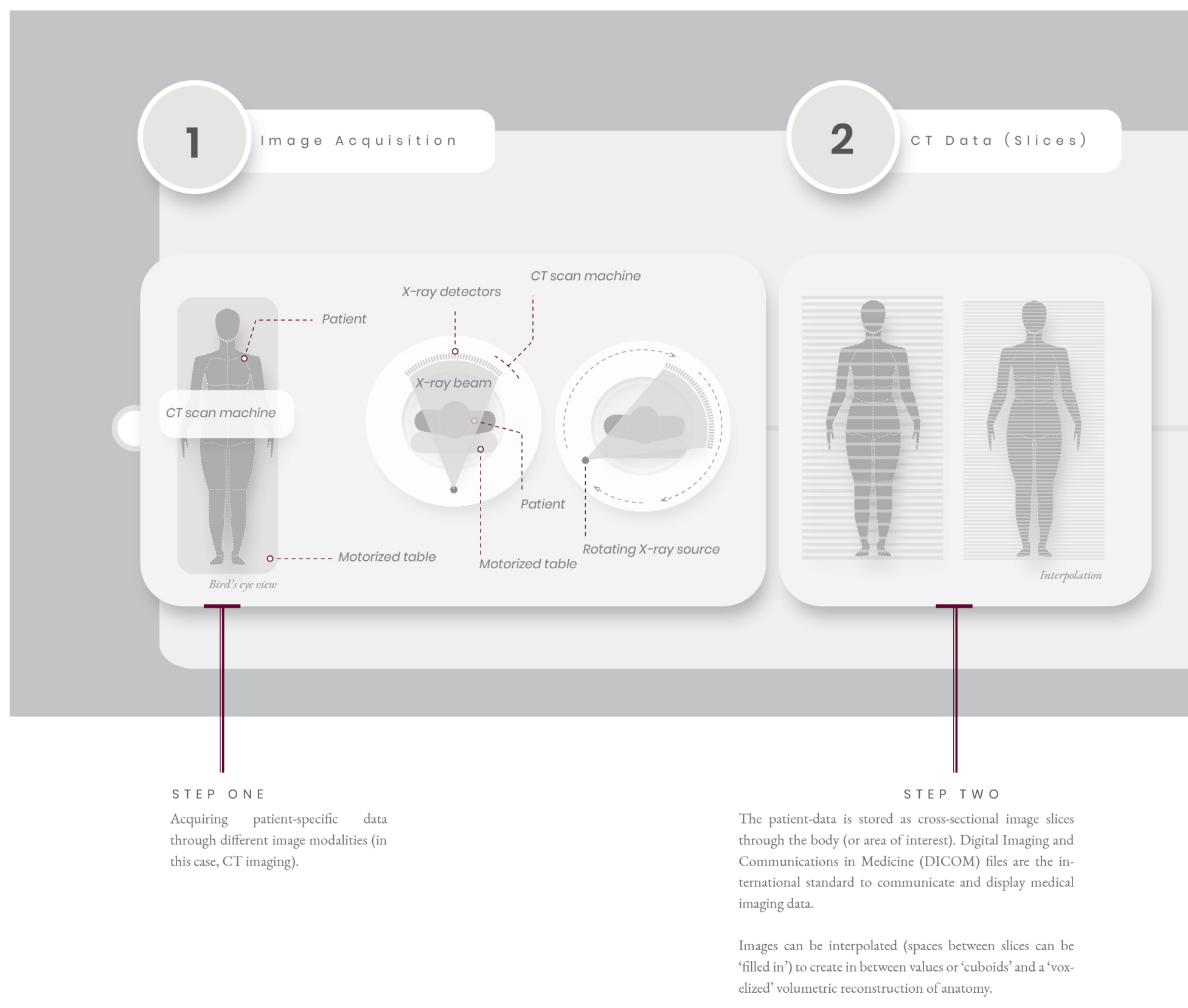




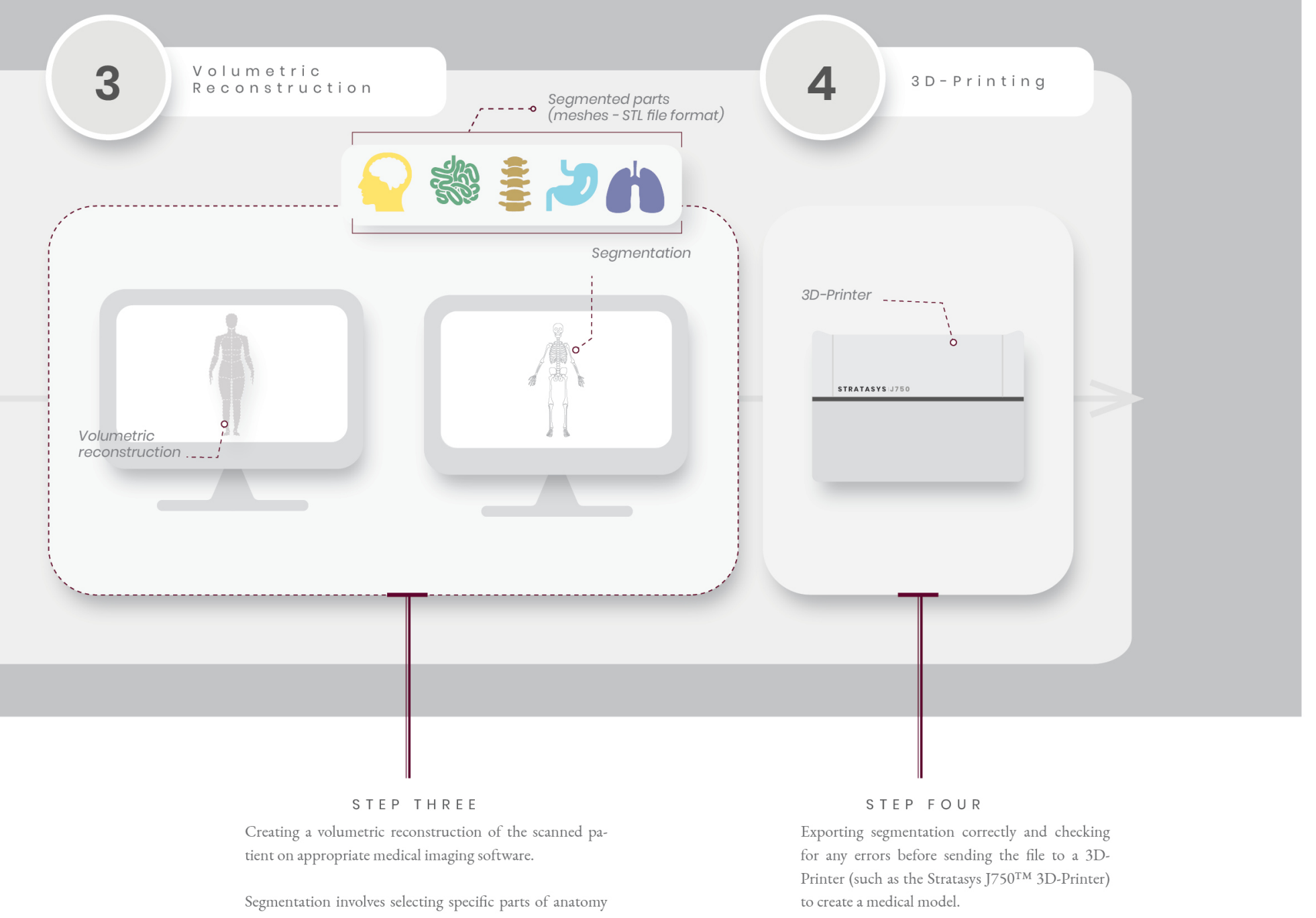

to be extracted from scan data. For example, the skeleton (bone). 


\section{Doctor-Patient Communication and Giving Informed Consent}

3D-printed medical models have shown to be useful tools for diagnosis, doctorpatient communication and informed consent practices. Recently, 3D-printed patient-specific medical models were used as tools in pre-operative patient consultation (Kim et al., 2019; Yang et al., 2018; Yoon et al., 2019). The models were compared with conventional communication methods such as verbal methods of communication and 2D CT images based on patients' understanding of their medical procedure (Kim et al., 2019; Yang et al., 2018; Yoon et al., 2019).

The 3D-printed models were used to communicate anatomy and physiology, illness characteristics, the surgical procedure and surgical risks. In all cases, the use of 3D-printed models was associated with better patient comprehension for informed decision making during the consultation process before surgery (Kim et al., 2019; Yang et al., 2018; Yoon et al., 2019). Using 3D-printed models during the pre-operative consultation process was associated with higher patient satisfaction, involvement and reassurance - contributing to the body of evidence that 3D-printed models could be useful in the routine clinical process aiding doctor-patient communication (Kim et al., 2019). doctor

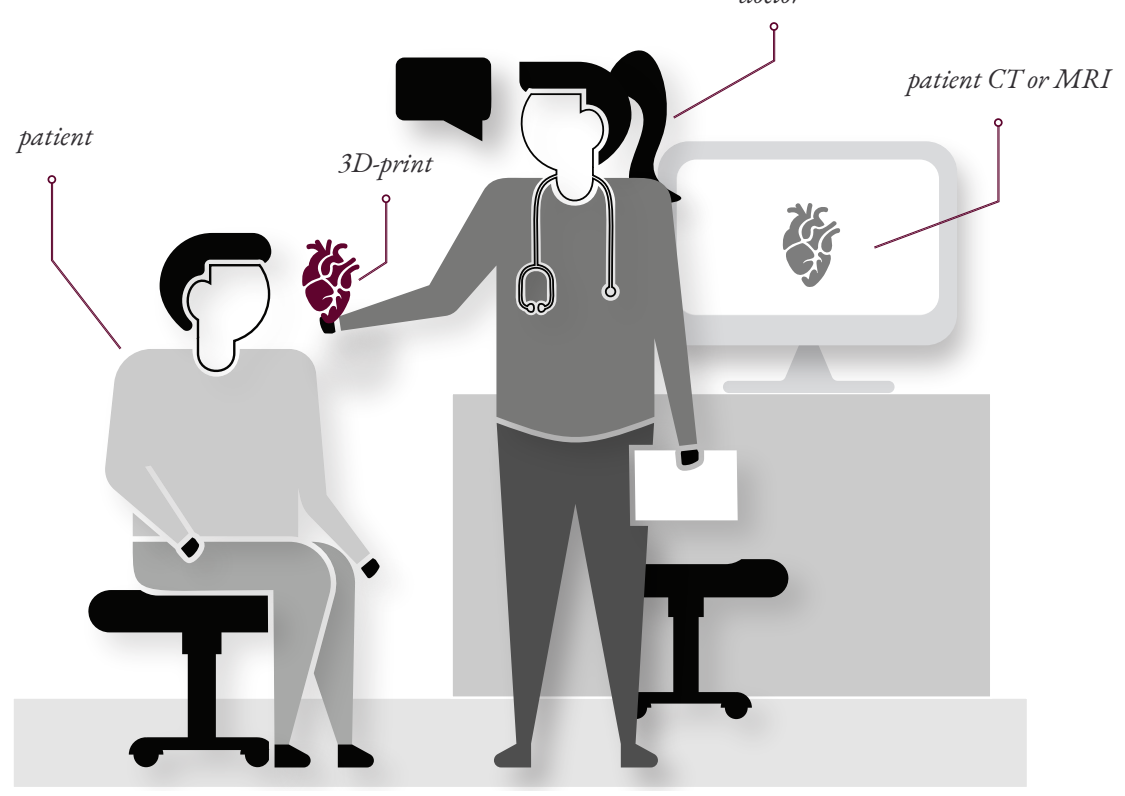

Figure 26. Doctor-patient consultation with 3D-printed model. 


\section{Medical Education and Research}

\section{Cadavervs. 3D-Print}

Studies have shown that medical students respond positively to 3D-printed anatomical models for educational purposes, such as anatomical teaching (Kim et al., 2019; Young et al., 2019). Historically, anatomy has been taught through cadaver dissection and anatomy textbooks. Although extremely important, there are restrictions surrounding using these educational tools in isolation. Cadavers have been criticised for their cost, limited availability, lack of portability, lack of patient-specific anatomy, health risks (such as disease transmission), and because of cultural and ethical concerns (Aziz \& McKenzie, 1999; Lim et al., 2016; Palter \& Grantcharov, 2010; Young et al., 2019; Zhang et al., 2008). A patient-specific synthetic model offers much of the same experience and knowledge, without the ethical and practical concerns.

Studies have shown that learning anatomy with 3D-printed anatomical models does not disadvantage students (Lim et al., 2016; Lioufas et al., 2016; Young et al., 2019). A study conducted by Lim et al. (2016) concluded that students who learnt anatomy on 3D-printed models retained higher amounts of correct information about anatomy compared to students who learnt anatomy on cadaveric material. Interestingly, this study concluded that 3D-prints might be an appropriate supplement to cadaver-based education, even providing some benefits over cadaveric materials.

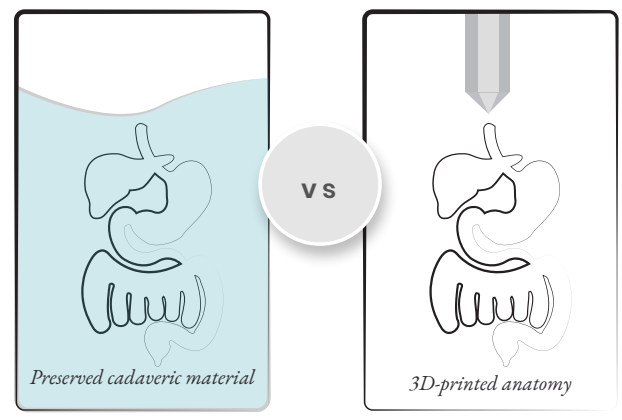

Figure 27. Cadaver vs. 3D-print.

\section{Embryology Education}

Medical bioimaging data is currently being used as a tool for teaching anatomy. This is because cadavers often do not show different ages, anomalies, normal anatomy and variations needed to understand anatomy completely (Rengier et al., 2010, p. 340). Preserved human embryonic and foetal specimens are not commonly available to most medical students due to legislative restrictions and the inherent sensitivity surrounding premature neonates and presenting foetal specimens as teaching materials (Young et al., 2019).

3D-printed embryonic, neonatal and paediatric anatomy can be produced from bioimaging datasets and used as teaching tools. Young et al. (2019) CT imaged and 3Dprinted an archived series of preserved human embryonic and foetal specimens for the purpose of medical education. The virtual reconstructions and subsequent 3D-printed models allowed embryology students to have access to foetal anatomical specimens. The ability to interact with these $3 \mathrm{D}$ models without worrying about having an adverse reaction or damaging original the specimens has drastically improved this area of embryology education (Young et al., 2019, p. 90).
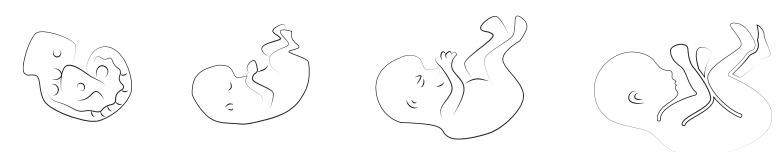

Figure 28. Foetal development. 
MEDICAL MODELLING APPLICATIONS

\section{Device Testing, Surgical Tool Development, Implants and Prosthesis}

3D-printed medical models are suited to the testing of medical devices that are worn both outside and inside the body. Developing technologies and medical devices involves an iterative design process. Testing devices and tools on medical models means that device limitations, failures and successes can be identified and solved before clinical trials begin, decreasing overall expenses and safety threats for patients (Ionita et al., 2014, p. 1).

3D-printing can tailor to the full anatomic variation of the human body and individual patient circumstances. The production of patient-specific biomedical devices, including implants and prosthetics, can be custom-made for each patient. Custom-made implants come in extremely useful for patients that lie outside the "standard" range concerning implant size and individual anatomical needs (Rengier et al., 2010). For example, medical modelling can be particularly useful in the area of auricular prosthesis for people with ear deformities. By acquiring medical data from the "normal" ear, a replica ear can be 3D-printed to help create a patient-specific ear prosthetic that is an exact match to the existing ear (Lin et al., 2018).

Medical modelling can help to prepare for invasive procedures that are always unique to each patient and can create custom-made implants to fit defective areas during the surgical reconstruction process. Many factors, including injury, disease and tumours, can cause anatomical deformities (Jardini et al., 2014). Different facial features and skull geometries can make craniomaxillofacial reconstructive surgery extremely challenging. 3D-printed models have helped with pre-operative planning and have enabled the simulation of osteotomies (Mavili et al., 2007). For example, a patient suffered from severe facial deformities after a cycling accident and 3D-printing using direct metal laser sintering (DMLS) techniques created a patient-specific cranial implant to reconstruct the patient's skull (Jardini et al., 2014). 


\section{Pre-Operative Planning \& Intra-Operative Guidance}

Medical modelling is used for teaching technical medical skills (Palter \& Grantcharov, 2010). Traditionally, the act of learning and practising new skills such as suturing, phlebotomy and intubation have occurred on patients - largely due to a lack of alternative teaching tools (Reznek et al., 2002; Severseike et al., 2019). This process affords medical students limited opportunities to practice procedures and learn from their mistakes, resulting in decreased patient safety and increased procedure risk (Reznek et al., 2002, p. 79).

3D-printed models can increase the understanding of complex patient pathologies and improving technical skill competency (Rengier et al., 2010). Studies validate the notion that $3 \mathrm{D}$-printed anatomical models contribute to personalised medicine, surgical planning and education across medical specialties and are rapidly changing the landscape of clinical practice (Jardini et al., 2014; Rengier et al., 2010; Yoo et al., 2016).

Planning procedures pre-operatively includes steps such as surgical incision, approach, hardware sizing and placement. Pre-operative planning can be helpful for complex maxillofacial and laparoscopic surgery (Kumara, 2012; Rengier et al., 2010). Operating strategy can be determined before the surgery, and subsequently, surgeons can enter procedures with more confidence (Rengier et al., 2010). 3D-printed models of patient anatomy can be valuable for complex patient cases where relationships between anatomical structures are hard to visualise through virtual mediums.

Cromeens et al. (2017) compared paediatric surgeons' understanding of complex connections between pygopagus conjoined twins when using a virtual reconstruction and a 3D-printed model. The authors concluded that the $3 \mathrm{D}$ printed model was significantly more helpful for understanding spatial relationships between structures, along with scale, shape and identifying the correct anatomical features when compared to a virtual anatomical reconstruction. The authors concluded that not only was more knowledge acquired and retained, but visuospatial information was learnt faster when using a 3D-printed model. 
MEDICAL MODELLING APPLICATIONS

\section{Surgical Simulation}

Simulation has come to the foreground as a method of teaching technical skills to surgical trainees. Practising and preparing for surgical procedures is possible with high-fidelity surgical simulators. Simulation-based training can immerse a trainee into a realistic and hands-on training situation (Cavaleiro et al., 2008). Surgical simulators offer the opportunity for surgical trainees to practice in a safe environment, receive valuable feedback without risking the safety of a patient, and can reduce the overall operating time (Barber et al., 2018; McGurk et al., 1997; Scerbo et al., 2006). 3D-Printed simulators have shown to be useful for communication, leadership and teamwork - skills which are an integral part of operative success (Palter \& Grantcharov, 2010, p. 1191).

\section{Neonatal E̊ Paediatric Simulation}

Studies have indicated that paediatric and perinatal acute care could benefit from simulators, especially in neonatal resuscitation and contributing to the success of a procedure (Barsness et al., 2013; Meurs et al., 2003; Rovamo et al., 2011). Preserving the realism of a surgical procedure by using the appropriate medical tools can help with incision placement, the identification of anatomy and the dissection of relevant tissues (Thielen et al., 2019). Unfortunately, paediatric and neonatal surgery are fields with limited access to high-fidelity simulation tools that accurately reflect individual neonatal and paediatric anatomy (Barsness et al., 2013). However, there is a growing amount of evidence to suggest that 3D-printed models are useful for the planning of paediatric and neonatal procedures and practices including the management of paediatric airway obstruction (Cavaleiro et al., 2008).

Approximately $10 \%$ of all neonates are admitted to Neonatal Intensive Care Units (NICU) every year with problems relating to the airway, breathing and circulation (Rovamo et al., 2011; Thielen et al., 2019). In many cases, medical simulative models are used to practice these essential procedures. However, models presently available are of low-fidelity, unrealistic and the materials do not reflect the complexities of neonatal and paediatric anatomy - potentially confusing even experienced surgeons and clinicians (Rovamo et al., 2011; 
Thielen et al., 2019).

For example, high-resolution 3D-prints may also help to replicate intricate parts of neonatal anatomy such as the heart system including heart chambers and valves (Thielen et al., 2019). However, there are limitations in the current 3D-printing process, including the small number of flexible materials that can respond in a similar way to human myocardium or vessels (Yoo et al., 2016).

Neonatal simulators with higher levels of realism and physical fidelity have been shown to help advanced learners, such as clinicians and surgeons, develop technical skills (Barsness et al., 2013). Training neonatal skills with simulators with functional fidelity can optimise skill transfer by suspending disbelief, helping with remaining focussed and improving cognitive and psychomotor aspects of skill acquisition (Cavaleiro et al., 2008; Thielen et al., 2019).

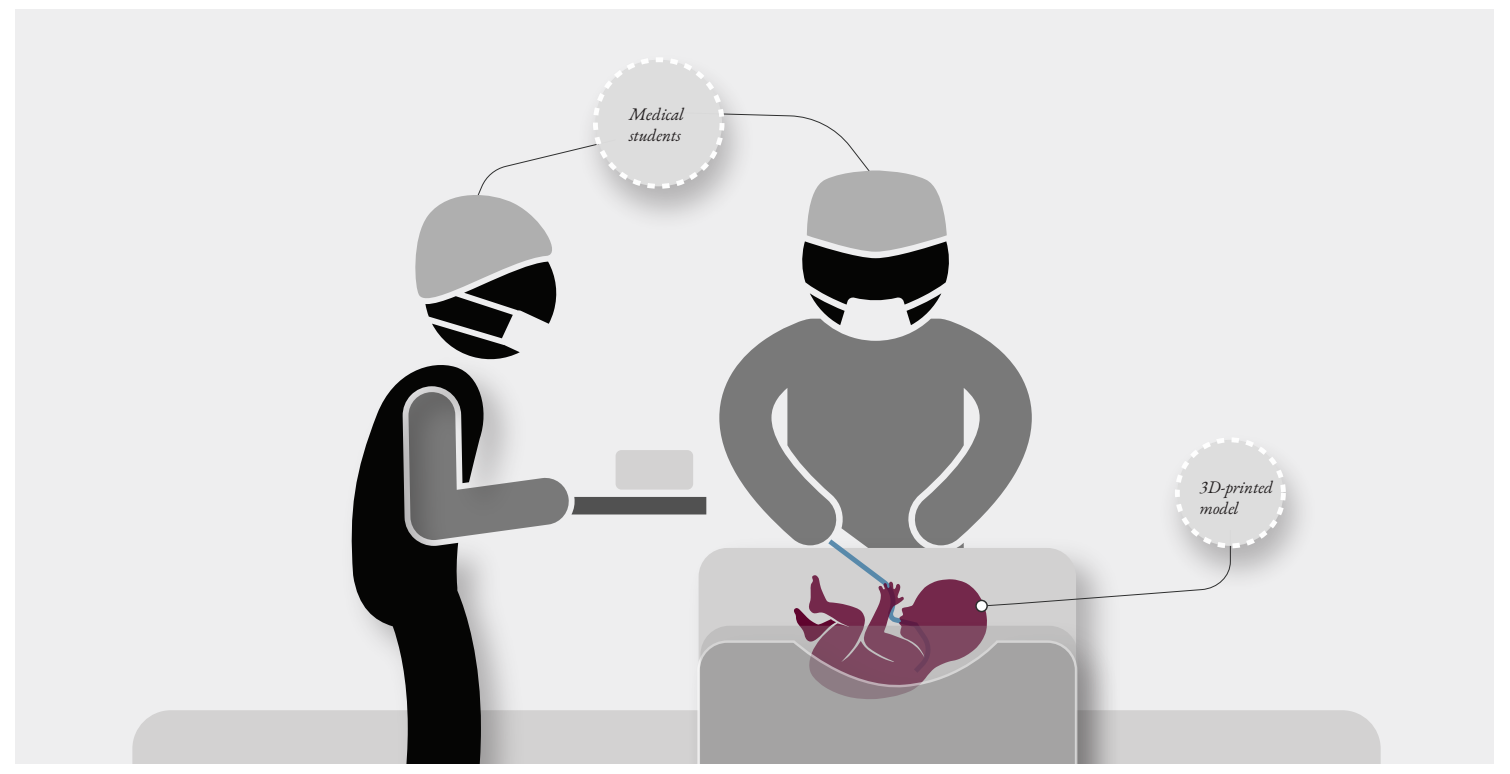

Figure 29. Medical students practicing a neonatal intubation procedure. 


\section{2 .3}

\section{MuIti-Material 3 D-Printing}

Overall, evidence suggests that multi-material 3D-printing produces smoother model surfaces at higher resolutions, allowing for more control over both internal and external structures (Brunton et al., 2015, p. 1). Multi-material 3Dprinting also has the potential to mitigate some of the frustrations caused by mono-material, low-resolution 3D-printers. This is because multi-material 3D-printing with photopolymeric materials can produce full colour and multidensity models in a single print (Kolb, 2017, p. 46).

Multi-material 3D-printing enables the fabrication of objects with heterogeneous material compositions allowing designers to "programme" detailed object functionality including anisotropic, colour and density property gradients (Doubrovski et al., 2015). Studies have shown that the multi-material Stratasys $\mathrm{J} 750^{\mathrm{TM}} 3 \mathrm{D}$-printer range exihibit great promise for high-resolution anatomical medical modelling because of the machine precision and ability to mimic human tissue and bone (Hosny et al., 2018; Lioufas et al., 2016; Severseike et al., 2019). The Stratasys J750 TM 3D-Printer is a full-colour and multi-material PolyJet 3D-printer that works by simultaneously and sequentially depositing tiny droplets (voxels) of different ultraviolet (U.V.) cured resins in 14-micron layers producing 3D-models with varied translucency, density and colour qualities (Bader et al., 2018, p. 2; Doubrovski et al., 2015).

Multi-material 3D-printers can process 3D-prints with "nestled" mesh-based (STL) file assemblies (where each STL is assigned with a different material quality). However, Hosny et al. (2018) highlight that creating "nestled" STL file assemblies can be complicated and costly - exhausting computer processing power. 


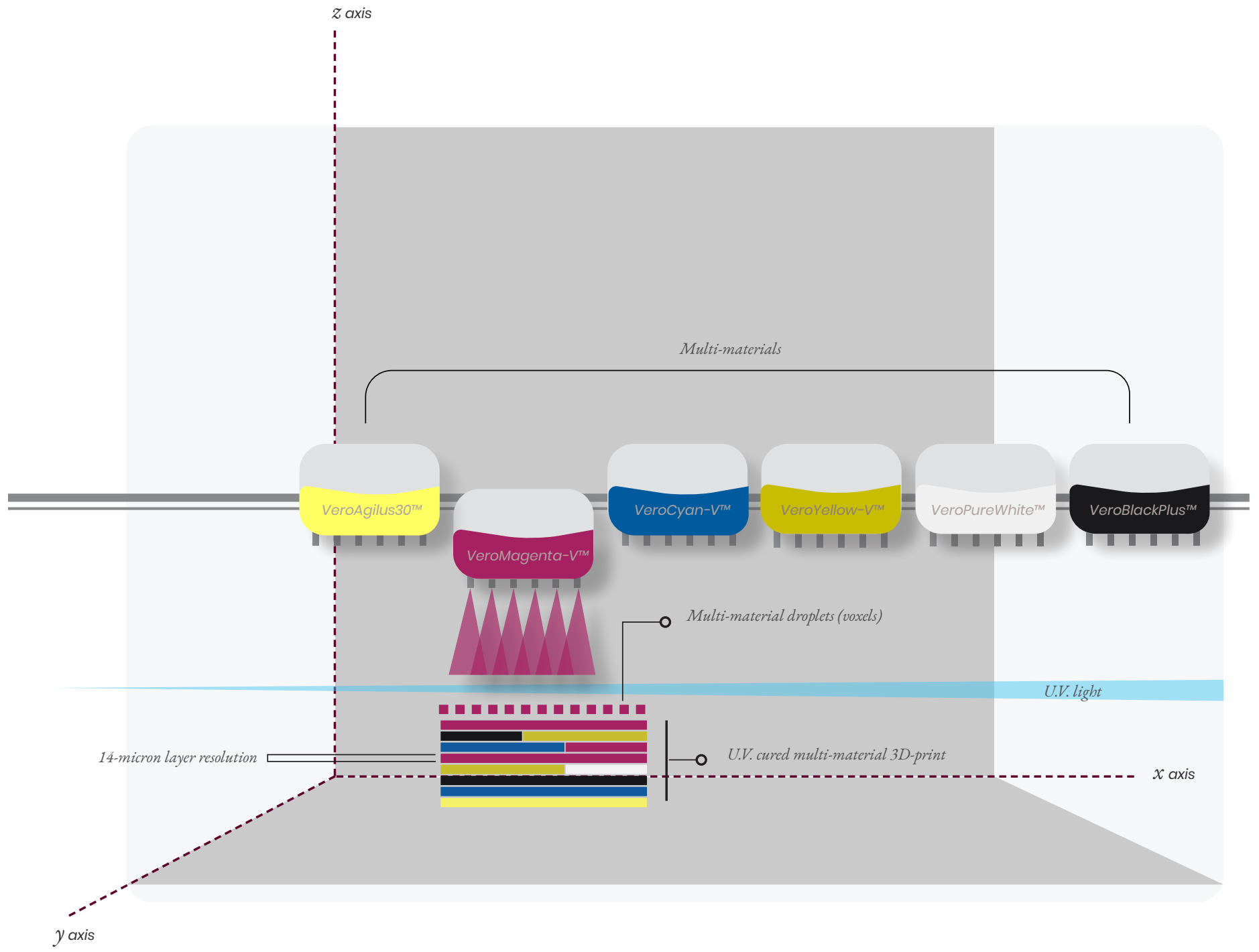

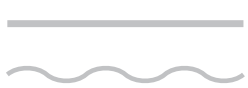

VARYING STIFFNESS

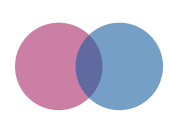

TRANSLUCENCY

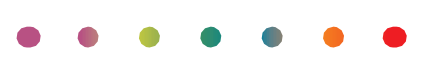

FULL-COLOUR

Figure 30. Multi-material 3D-printing on the Stratasys J750 TM 3D-Printer. 
Literature suggests exploring the potential of using a bitmapbased additive manufacturing workflow for medical modelling purposes. Many studies challenge traditional 3D-printing workflows and have documented a need for data-driven material modelling (Bader et al., 2016, 2018; Brunton et al., 2015; Doubrovski et al., 2015; Stute et al., 2018). Data-driven bitmap-based additive manufacturing - also referred to in the literature as "voxel printing” (Bader et al., 2018), "bitmap printing” (Doubrovski et al., 2015; Hosny et al., 2018), or "error-diffusion halftoning” (Brunton et al., 2015) - can translate information from various datasets into heterogeneous physical entities by converting on-screen pixels into voxels (Kolb, 2017) or 3Dpixels (Hiller \& Lipson, 2009). Each voxel is a homogenous and isotropic material (Sossou et al., 2019).

\section{How does bitmap-based 3D-Printing work?}

A bitmap-based 3D-printing workflow allows image files to be sent directly to some multi-material 3D-printers, such as the Stratasys $\mathrm{J} 750^{\mathrm{TM}}$ 3D-Printer for the production of physical forms. The Stratasys J750 TM 3D-Printer can 3D-print one image on top of another, producing 14-micron resolution voxel matrices. When images are deposited sequentially on top of each other, the 3D-printer can construct a tangible 3D model.

Bitmap-based 3D-printing works by generating a set of layers (multiple isosurfaces) in a bitmap file format which is the same resolution as the native 3D-printer $\mathrm{z}$ resolution (Bader et al., 2018; Brunton et al., 2015). Each image sent to the 3D-printer contains a pixel matrix inspired by error-diffusion algorithms from 2D-printing (Brunton et al., 2015). Due to the limited amount of 3D-printer primary colours, additively manufactured shades and gradients can only be produced through 2D-printing Halftoning principles (Bader et al., 2016, 2018;
Brunton et al., 2015; Doubrovski et al., 2015; Stute et al., 2018). So, just like a printed photograph which is comprised of many pixels, a 3D-print made up of many voxels can be observed as a homogenous area with blended materials even though each pixel is assigned with a primary 3DPrinting material such as VeroCyan-V ${ }^{\mathrm{TM}}$, VeroMagenta$\mathrm{V}^{\mathrm{TM}}$, VeroYellow-V ${ }^{\mathrm{TM}}$, VeroClear ${ }^{\mathrm{TM}}$, VeroBlackPlus ${ }^{\mathrm{TM}}$ and VeroPureWhite ${ }^{\mathrm{TM}}$ (Kolb, 2017). Each pixel within the matrix defines the material and placement specification of a $3 \mathrm{D}$-printed voxel allowing for the generation heterogeneous material composites (Bader et al., 2016, 2018). Heterogeneous compositions mean that bitmap-based 3D-prints can be composed of multiple materials that are fused together, differing in their chemical and physical properties (Doubrovski et al., 2015, p. 4). The variations in materials, including the creation of gradients in colour and density, allow for exploration opportunities, especially in the fields of biomimicry and medical modelling. A bitmap-based 3D-printing workflow has potential to completely bypass traditional meshbased segmentation methods.

There have been a limited amount of studies that explore the capabilities of bitmap-based 3D-printing techniques for purposes of medical modelling (Bader et al., 2018; Hosny et al., 2018). Huotilainen et al. (2014) suggest that there should be research conducted in the area of medical modelling, looking at the most accurate and feasible way of converting voxel-based DICOM data into 3D-printed medical models.

Overall, studies acknowledge that the strength of bitmapbased 3D-printing lies its accuracy, limitless manufacturing possibilities and the production of complex material combi- 
nations at a microscale. In saying this, voxel-based 3D-printing also comes with colour and material reproduction challenges based on the need to control the material of every voxel to create accurate material combinations without appropriate software tools available (Brunton et al., 2015; Stute et al., 2018).

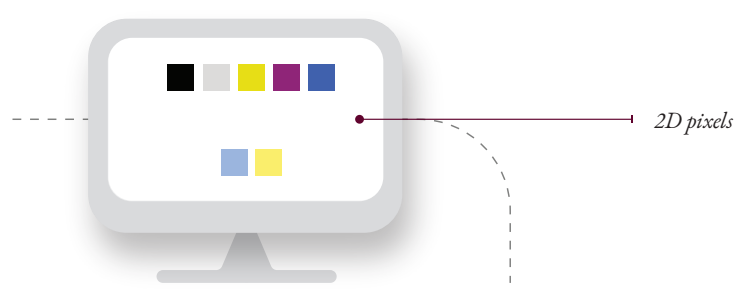

image/s (dataset) sent to 3D-printer
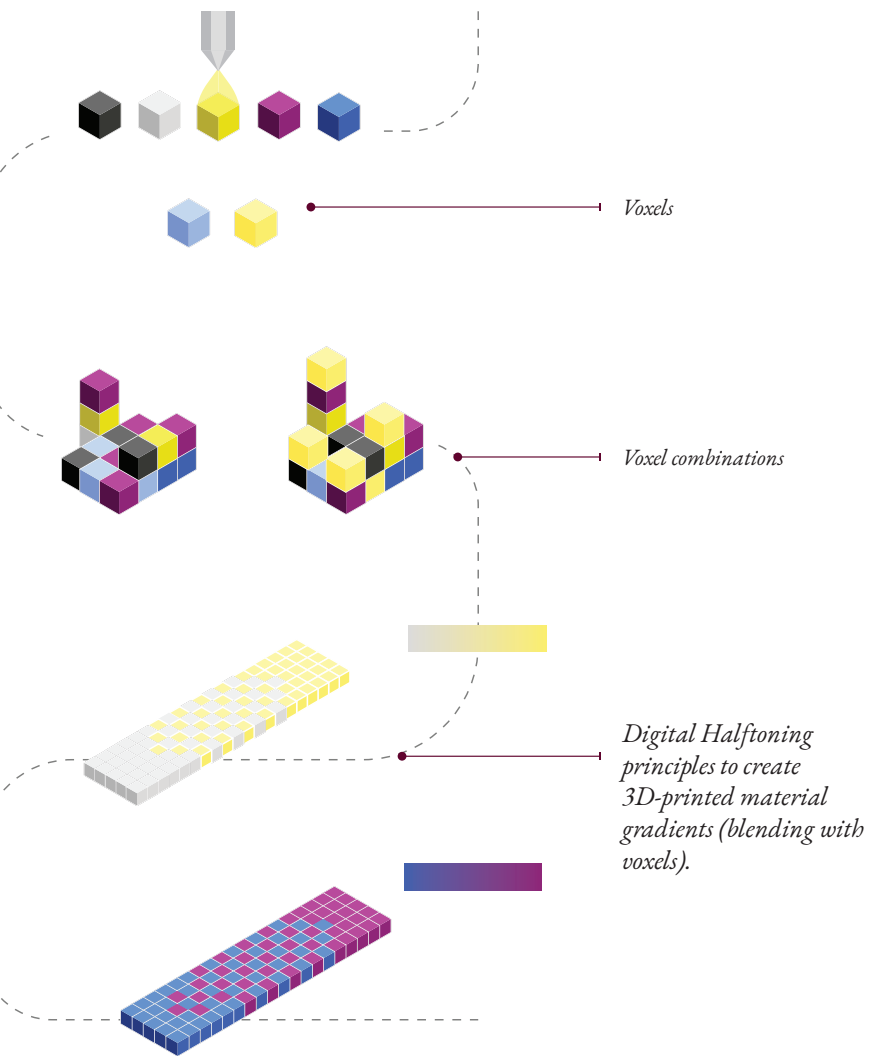

Figure 31. Converting on-screen pixels into 14-micron multi-material droplets (voxels). 


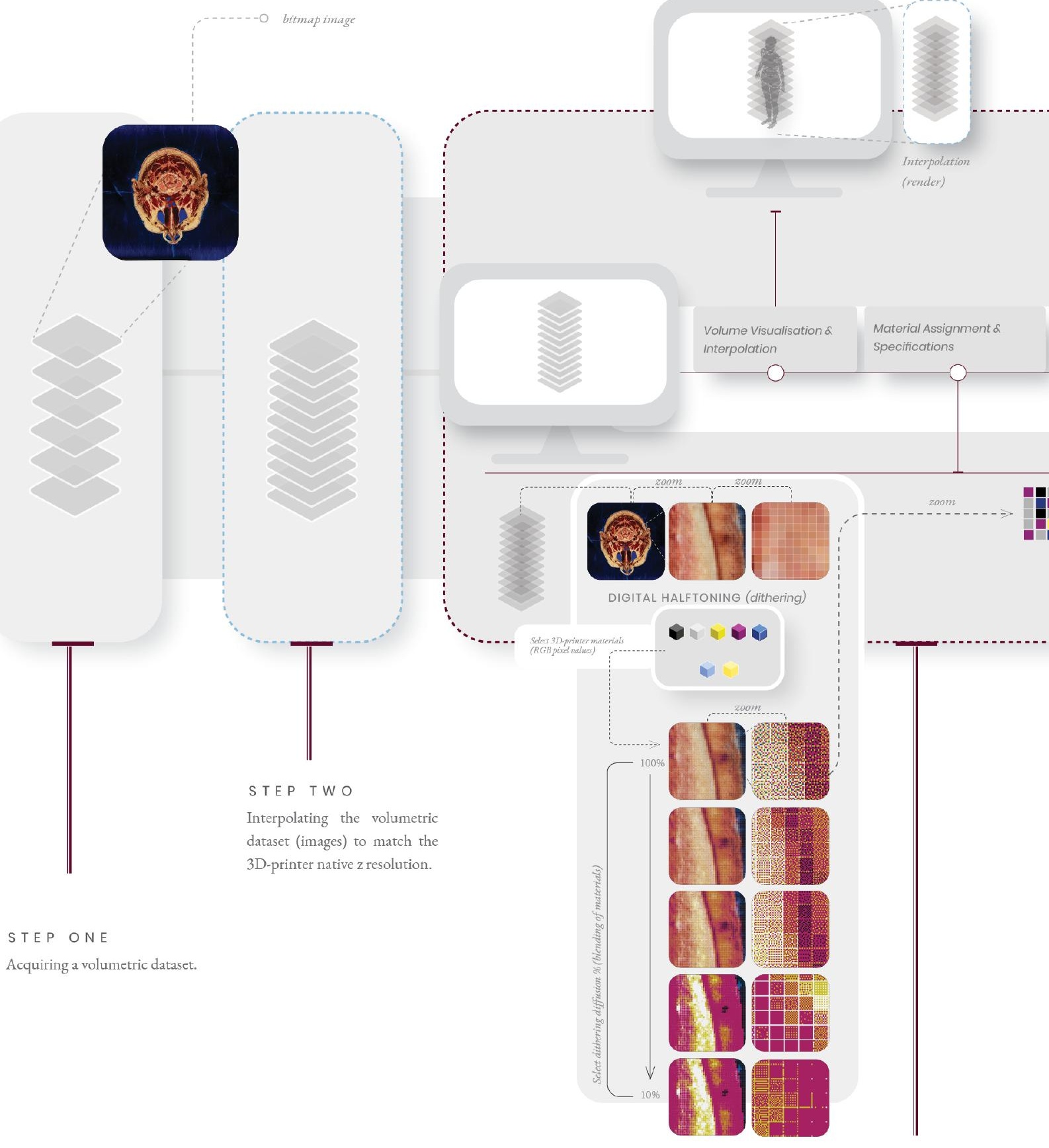

STEP THREE

Processing the images to create a bitmapbased 3D-printing file (defining 3D-print parameters and materials). This step may replace traditional segmentation processes. 


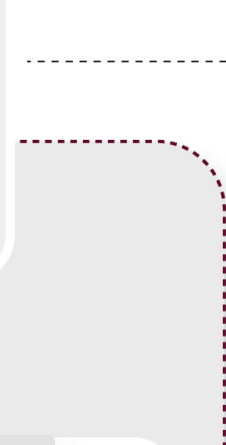

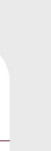
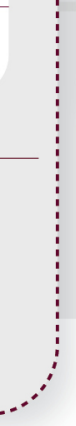

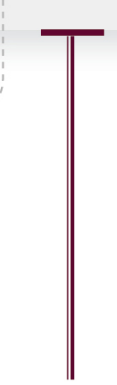

STEP FOUR

Processing the bitmap image stack through the GrabCAD Voxel Print Utility.

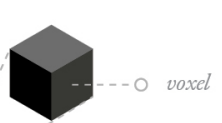

D-printed model

(1) GRABCAD
PRINT
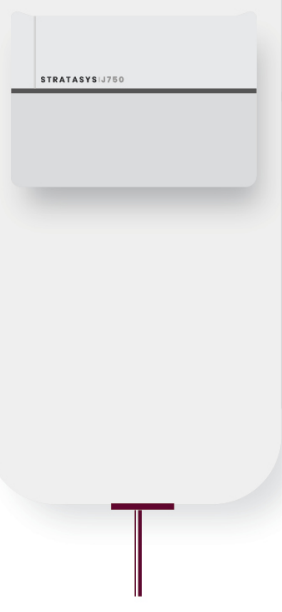

STEP FIVE

3D-printing the final model by sending GCVF file to a 3D-printer with bitmapbased (voxel) 3D-printing capabilities.
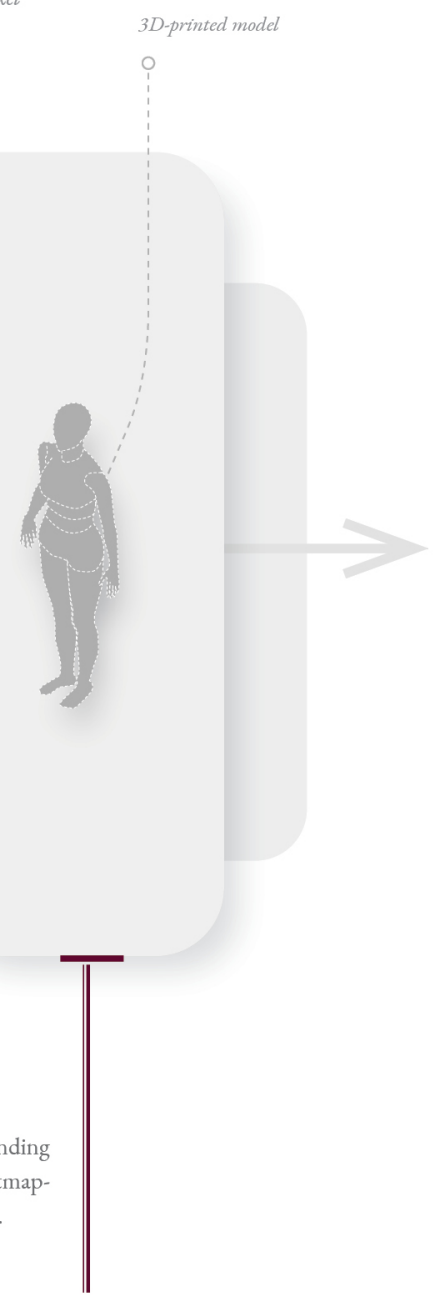

STEP SIX

Multi-property 3D-printed model with gradients in colour, density and translucency. 
THE GAP IN CURRENT KNOWLEDGE, THE PROPOSED RESEARCH \&

ITS ANTICIPATED VALUE

A plethora of literature suggests that 3D-printing technologies are advancing at a fast pace and provide ample benefits in the areas of medical education, giving informed consent, surgical simulation and pre-operative planning - serving as a possible valuable tool for clinicians, families and communities.

However, the cost efficiency and the degree of realism of current medical modelling workflows have an immense impact on the overall quality of a medical model and the ability to produce "fit for purpose" models efficiently. Limiting factors of current medical models include the lack of soft tissue realism, lowfidelity detail due to traditional segmentation techniques and the processing time involved in traditional mesh-based segmentation methods (Huotilainen et al., 2014; Mavili et al., 2007; Yoo et al., 2016).

\section{A Bitmap-based 3D-printing Workflow}

Some authors hypothesise that surgical training using high-fidelity medical models will eventually be an "essential component" in surgical training programmes and a "prerequisite requirement for performing the procedure on the patients" (Yoo et al., 2016, p. 10). Therefore, there is value in exploring a more sustainable, streamlined medical modelling workflow that can create patientspecific medical models to a higher degree of anatomical realism.

Novel bitmap-based 3D-printing workflows may offer interesting opportunities for medical modelling at a high-fidelity, with potential to mitigate some of the disadvantages of current medical modelling workflows using traditional mesh-based segmentation techniques. Current 3D-printed medical models are predominantly created to replicate mono-material bony body structures (Huotilainen et al., 2014, p. e260). However, material properties created through bitmap-based 3D-printing have potential to emulate bioinspired material qualities inherent in human anatomies such as both bone density, soft tissue and skin elasticity (which is almost impossible to mimic using any other 3D-printing technique) (Doubrovski et al., 2015; Oxman, 2017). 


\section{A Streamlined Workflow}

A streamlined medical modelling workflow has the potential to save time, money and produce highly accurate models with graduated densities, colour and flexion. An automated bitmap-based 3D-printing medical modelling workflow may produce a higher amount of patient-specific and high-resolution medical models at a lower cost by bypassing the need for current mesh-based segmentation methods and expensive CAD software. Therefore, there is immense value in establishing and exploring a bitmap-based medical modelling workflow and novel 3D-Printing technologies.

Parallels drawn between bitmap-based 3D-printing and bioimaging methods means that the conversion of bioimaging datasets to bitmap-based 3D-prints could be a promising direction for future research.

This research will build off knowledge from previous studies that suggest "generated stacks of binary bitmap files can be considered analogous to files produced in volumetric scanning technologies" (Doubrovski et al., 2015, p. 9).Both bitmap-based 3D-printing and biomedical imaging datasets are comprised of cross-sectional image stacks that create volumetric 3D forms. A CT scan can generate a voxel representation of the human body if axial slices are interpolated from one image to the next, forming volumetric pixels, also referred to as voxels (Bibb et al., 2015). Volumetric datasets composed of image stacks obtained from biomedical imaging can be used as direct 3D-printer inputs. A streamlined bitmap-based 3D-printing workflow may bypass the conversion steps of traditional segmentation, resulting in the preservation of anatomical detail and a completely automated manufacturing process - without the need for mesh-based file preparation. 


\section{Gap in Knowledge}

Studies have demonstrated that biomedical imaging data can be utilised for the fabrication of medical models with gradated colour using a bitmap-based 3D-printing workflow (Bader et al., 2018; Doubrovski et al., 2015; Hosny et al., 2018; Kolb, 2017). However, bitmap-based 3D-printing is still novel, and its potential for medical applications has not yet been explored in depth, leaving considerable gaps in knowledge about the capabilities of bitmap-based 3D-printing for medical modelling.

Whether or not anatomically accurate and patient-specific colour or biomechanics can be mimicked through a bitmapbased 3D-printing medical modelling workflow is relatively unknown. Current bitmap-based 3D-printing research has produced 3D-printed models that successfully graduate material colour and opacities (Vero ${ }^{\mathrm{TM}}$ ) but limited exploration has been conducted into the gradation of 3D-printed material densities or 'stiffness' (Agilus30 ${ }^{\mathrm{TM}}$ ) and the reproduction accurate anatomical colours (Bader et al., 2018; Hosny et al., 2018).

Bitmap-based 3D-printing has the potential to produce bespoke pathological models which vary in both chemical and physical properties (Doubrovski et al., 2015). Research should be conducted into streamlining the mesh-based traditional segmentation workflow by exploring the possibilities of bitmap-based 3D-printing for high-fidelity medical modelling. Further research could properly quantify the value of a bitmap-based medical workflow to the healthcare sector.

\section{The Proposed Research}

This research portfolio will creatively explore how bitmapbased 3D-printing can produce medical models from biomedical imaging datasets with graduated stiffness that mimic anatomical densities as well as anatomically accurate colours. Manufacturing on medical models at a 14-micron voxel resolution may provide another way of viewing and understanding the human anatomy, adding to existing knowledge about human physiology.

This research will introduce a bitmap-based additive manufacturing file preparation workflow that has potential to convert biomedical imaging data into multi-property and full colour $3 \mathrm{D}$-printed synthetic anatomy in minutes. The hope is that this research will act as an introduction to bitmap-based 3D-printing techniques for medical applications and serve as a foundation for future studies that quantify the value of bitmap-based 3D-printing for medical applications. This research will bring to light the advantages and disadvantages of bitmap-based 3Dprinting workflows for different medical applications. 


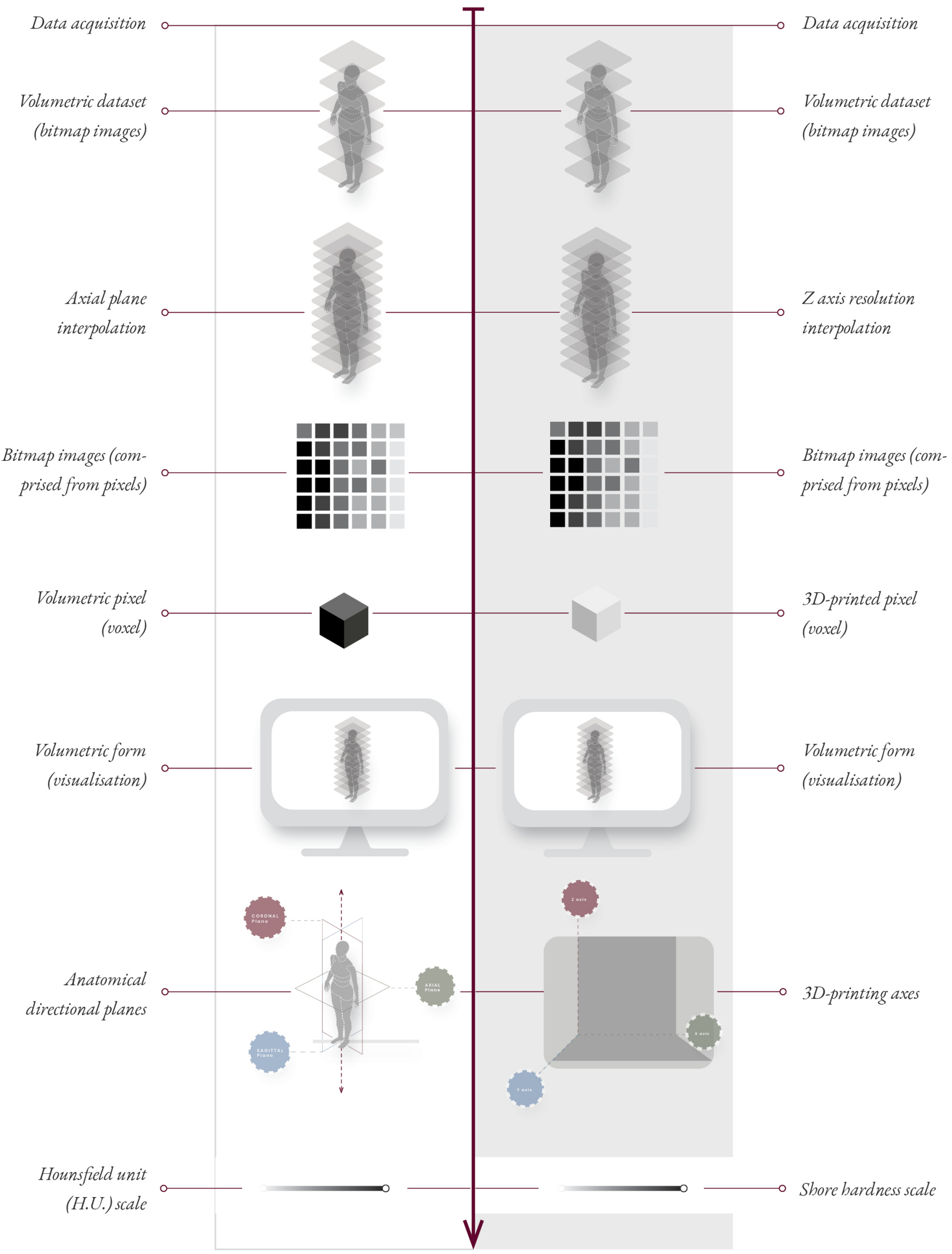




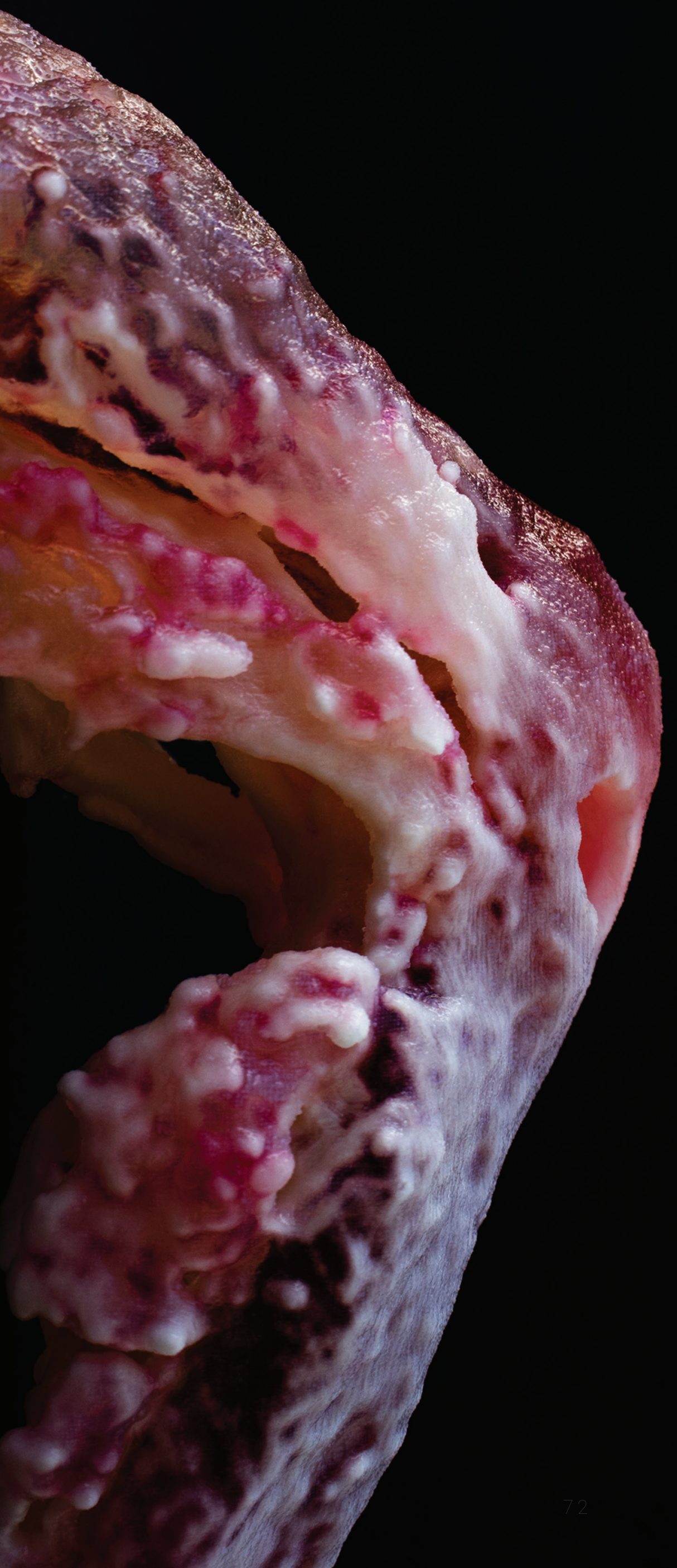




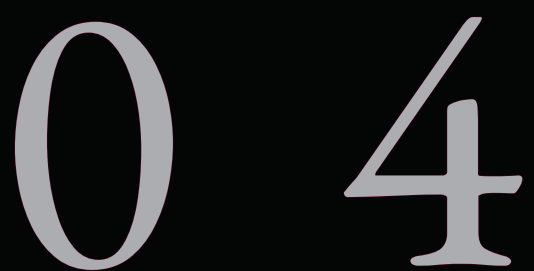

PRACTICALACTION RESEARCH

Left:

Figure 34. Bitmap-based 3D-printed neonatal hip. 
PRACTICALACTION RESEARCH KEY

MATERIAL COLOURS (RGB)

VeroPureWhite ${ }^{\mathrm{TM}}$

VeroCyan-VTM

VeroMagenta-VTM

VeroYellow-VTM

VeroBlackPlus $^{\mathrm{TM}}$

VeroClear $^{\mathrm{TM}}$

Agilus30 $30^{\mathrm{TM}}$

SUP706 $6^{\mathrm{TM}}$ 


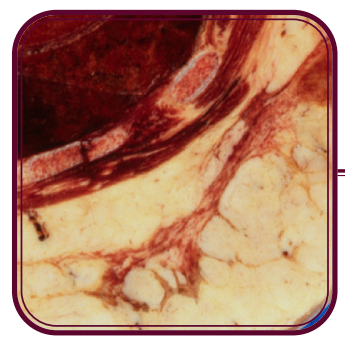

Original bitmap image

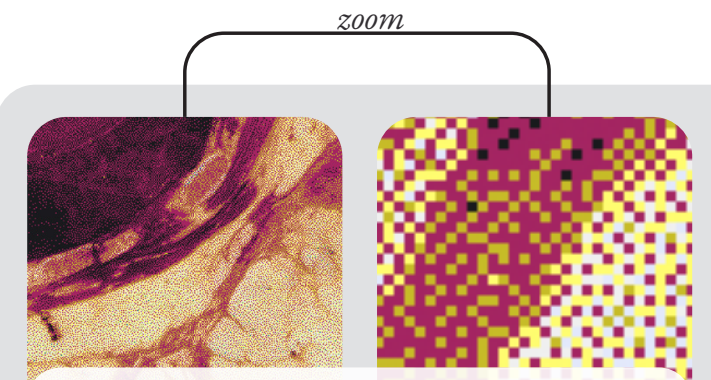

100\% diffusion
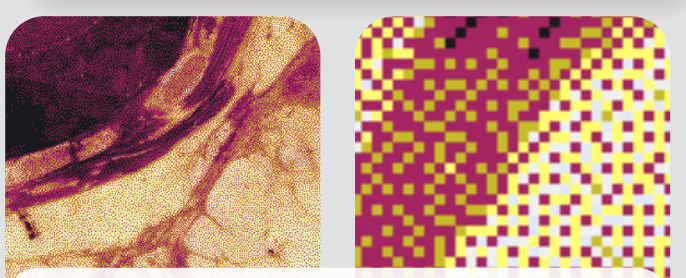

90\% diffusion

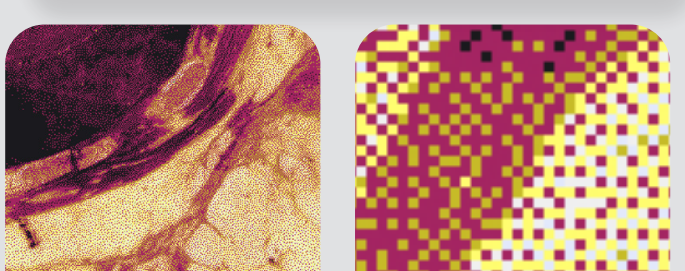

$80 \%$ diffusion
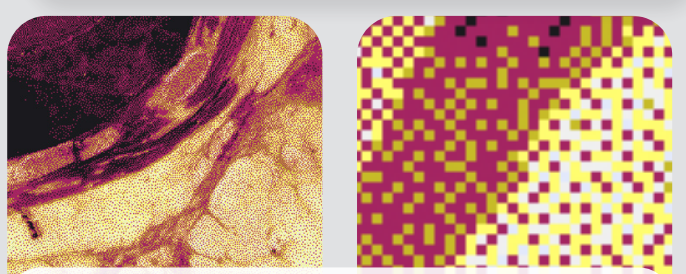

70\% diffusion
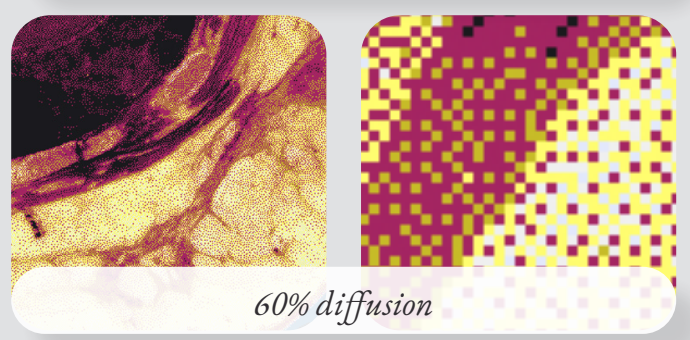
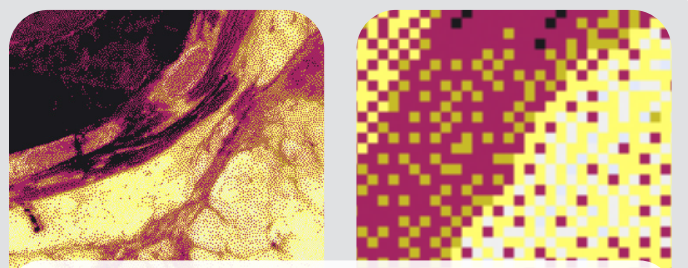

50\% diffusion

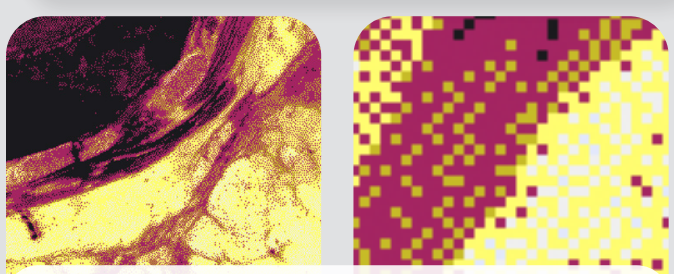

$40 \%$ diffusion

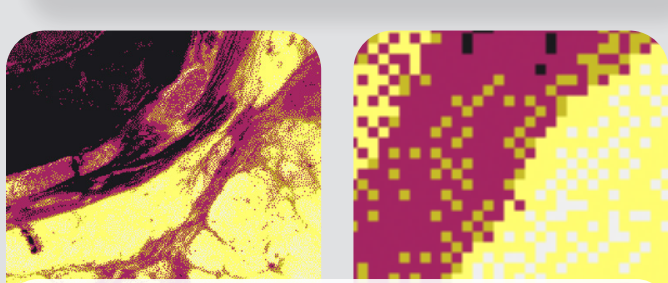

$30 \%$ diffusion

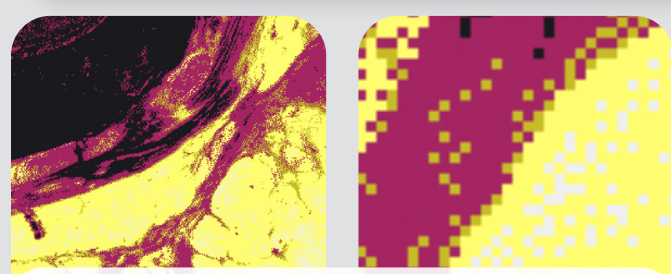

$20 \%$ diffusion

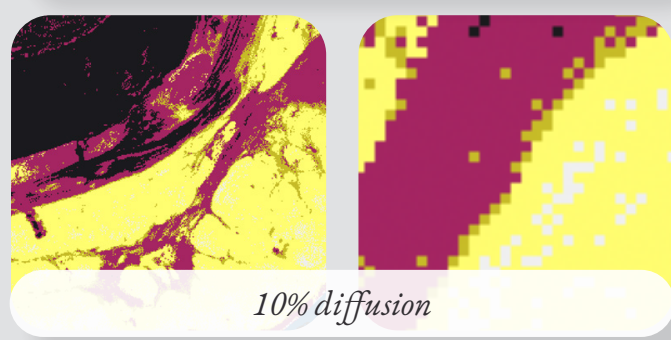

Figure 35. Practical action research cycle key (material colours and digital halftoning). 


\subsection{A S E S T U D Y 1}

3D-Printing a Full-Colour Voxel Human using the Visible Female Dataset (Visible Human Project ${ }^{\circledR}$ ) and a Bitmap-based Additive Manufacturing Workflow.

Left:

Figure 36. Digitally halftoned image of the Visible Female dataset from the Visible Human Project ${ }^{\circledR}$. 
Case Study 1 focused on developing a streamlined bitmap-based additive manufacturing workflow to produce an anatomically accurate, high-fidelity, and full colour model of the Visible Female ${ }^{\circledR}$. The 3D-printed model of the Visible Female ${ }^{\circledR}$ was produced by using serially sectioned cryosection images of a female cadaver produced by researchers working on the Visible Human Project ${ }^{\circledR}$ (VHP). The original cryosectional images at $0.33 \mathrm{~mm} \mathrm{XY}$ resolution generated cross-sectional images of anatomy, at the Stratasys J750 ${ }^{\mathrm{TM}}$ 3D-Printer native z (axial) resolution, meant that three-dimensional forms could be additively manufactured from bitmap images.

Initial parallel prototyping in Case Study 1 was conducted in another School of Design Innovation course at the Victoria University of Wellington, titled CCDN444. The final design workflow was developed based on the information gathered from the CCDN444 project. The developed workflow presented in this research produced a high-fidelity bitmap-based 3D-print of the Visible Female®.

The Visible Female®'s cause of death was heart disease, induced by morbid obesity (Hamzelou, 2015). Subsequently, the Visible Female®'s body was donated by her husband to the VHP (Hamzelou, 2015). Male and female datasets from the VHP were recorded through high-resolution photographic images and biomedical imaging including CT and MRI by the University of Colorado Health Sciences Centre, along with the National Centre for Atmospheric Research. Serial sectioning, photographing and segmenting this data has led to 3D-reconstructions of the Visible Human ${ }^{\circledR}$ - which has been employed in medical education and utilised in clinical practice (M. Chung \& Park, 2007).

To traditionally convert serially sectioned slices into a file suitable for 3D-printing, certain anatomical features must be segmented and converted into mono-material boundary meshes. Current studies have not taken advantage of high-resolution multi-material 3D-printers that allow for control over material droplets or 'voxels' to produce highly-realistic models of Visible Human Project ${ }^{\circledR}$ anatomy (M. Chung \& Park, 2007; Kirana P. Kumara, 2012). 


\subsection{2}

\section{Criteria for case study 1}

The focus of Case Study 1 was to produce a bitmap-based 3D-printing workflow by creating a series of design experiments utilising the full-colour Visible Female ${ }^{\circledR}$ dataset, with a focus on replicating accurate anatomical colour. Focussing on gradients in colour (rather than gradients in density) using a bitmapbased 3D-printing working was a good starting point for investigations. Experimentation with scale was conducted to test the consistency of interpolation functions as well as how scale affected 3D-print fidelity.

C RITERIA

\section{Explore and develop a bitmap-based 3D-printing workflow.}

A bitmap-based 3D-printing workflow should be developed to 3D-print models of human anatomy with anatomically accurate colour. The workflow should be streamlined and work to create extremely detailed 3D-prints using a small amount of processing steps and programmes.

\section{Show the human body at a high-fidelity and with realistic colour.}

The bitmap-based 3D-printing workflow should produce 3D-prints constructed using the Visible Female ${ }^{\circledR}$ dataset. Using parallel prototyping, the preferred workflow will be chosen based on the quality of the resulting $3 \mathrm{D}$ prints. These 3D-prints should preserve the most amount of original data possible.

\section{Use the most effective bitmap-based 3D-printing workflow to create} a final, high-fidelity model using the Visible Female ${ }^{\circledR}$ dataset.

The level of realism in the Final Design should be unprecedented to provoke thought about the future of 3D-printing in medicine and the contextual applications of the developed bitmap-based 3D-printing workflow. The final 3D-print should be evaluated by a process of self-reflection and discussion with expert opinions. 


\section{File preparation}

DATA ACQUISITION

A dataset is required to begin bitmap-based 3D-printing. High-resolution imaging data, provided by the VHP, was used to produce full-colour and highresolution 3D-prints from a set of images. The VHP data was selected to test this novel bitmap-based workflow because of the open-source, diverse and high-fidelity nature of the imaging. 5,189 cross-sectional images of the Visible Female were downloaded and used for Case Study 1.
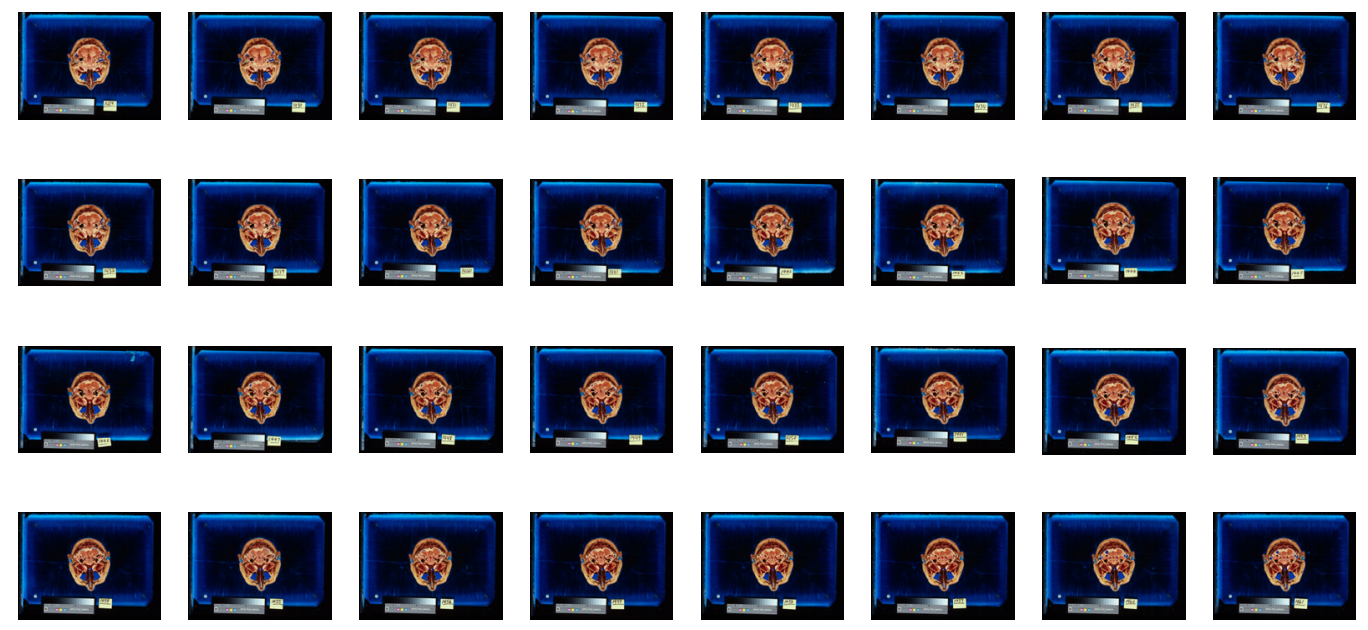

Figure 37. Cross-sectional images of the Visible Female®.

DIGITAL WORKFLOW

The Visible Female ${ }^{\circledR}$ dataset was volumetrically reconstructed to create a virtual model. The virtual model was scaled down during parallel and final design prototyping for cost saving purposes and an interpolation function was used to fill in missing slices to match the native 3D-printer $\mathrm{z}$ (axial) resolution. The prototyping process followed the workflow outlined in Figure 38. 


\section{FOLDER 1}

Download volumetric data in a folder

(VHP cross-sectional images)

\section{STAGE ONE: Volumetrically}

Reconstructing Medical Data

Enter volume reconstruction software.

Volumetrically reconstruct model.

Scale model (select scale).

Interpolate images to create a virtual model with smooth surfaces (also known as a voxel model).

Export Images as PNG (bitmap) image files at the native 3D-printer $z$ resolution. In the case of high-mix 3D-printer settings, export cross-sectional images slices at $0.027 \mathrm{~mm}$ intervals. For high-resolution 3D-printing, export images at $0.014 \mathrm{~mm}$ intervals.

\section{FOLDER 2}

Save the images in a folder on the computer.

Decide on the area of anatomy to 3D-print.

\section{FOLDER 3}

Drag images that include the area

on anatomy (eg. brain) into a

separate folder to process for automated batch processing.

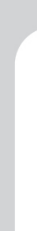

STAGE TWO: Batch Processing

(automated workflow)

Enter batch processing software.

Open the actions tab and create a new action.

- Name Action.

Open an image from FOLDER 3 (select the

image with the largest surface area)

Crop image to include the boundary box (this can be a general boundary box or the 'Subject' or 'Outline' of the subject matter).

- Delete blue background (make sure that everything is deleted apart from the colour aspect of the 3D-Print).

- If applicable, you can scale the image in this step.

- Image $\longrightarrow$ Image size $\longrightarrow$ change the image size to 3D-printer $\mathrm{XY}$ resolution.

- Assign the 3D-printer materials by a process called Digital Halftoning (dithering).

- If you want a clear bounding box.

0

- If you do not want a clear bounding box.

\section{FOLDER 4}

- Save the image as a PNG file (Save as $\longrightarrow$ Create a new folder for the digitally halftoned images and save in this folder).

- Close.

Select Don't Save.

Automate the Batch Process to Digitally Halftone all images.

File $\longrightarrow$ Automate $\longrightarrow$ Batch Process.

Select Action.

Select FOLDER 3

- Automate. 
Removing the blue background:

Try the following:

Select $\longrightarrow$ Subject

Select $\longrightarrow$ Focus

Select $\longrightarrow$ Colour range $\longrightarrow$ Blue

\section{- Enter the RGB values for primary}

$3 D$-printer colours utilised.

Select dithering and diffusion options.

Create new layer.

Drag to be Layer 1.

With the paint bucket tool, paint the layer with the VeroClear ${ }^{T M}$ colour value.

\section{Create new layer.}

- Drag to be Layer 1.

- With the paint bucket tool paint the layer with the Support Material (SUP706 TM) colour.
STAGE THREE: Rename Files for

\section{Software Compatibility}

Open file naming software.

Select all images in FOLDER 4

Batch Rename.

PrintName_1 (for one digit numbers)

PrintName_10 (for two digit numbers)

PrintName_100 (for three digit numbers)
STAGE FOUR: Prepare File for Printing

\section{on GrabCAD Print Voxel Print Utility}

Enter GrabCAD Print and prepare files for 3D-Printing.

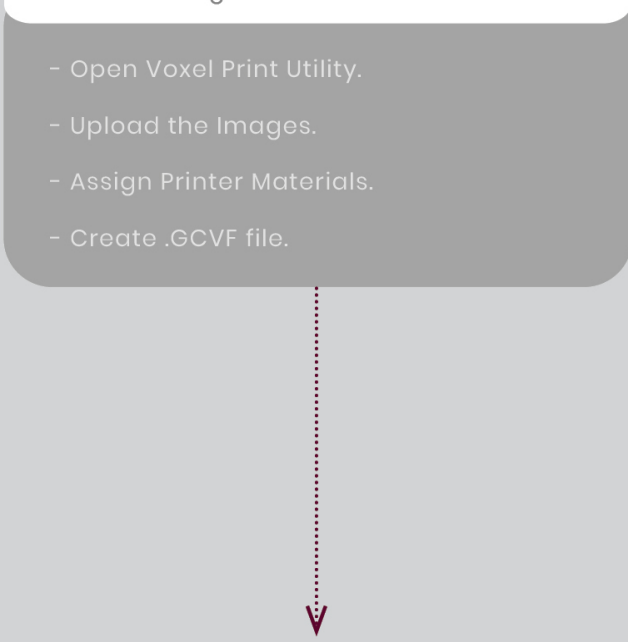

STAGE FIVE: 3D-Print 

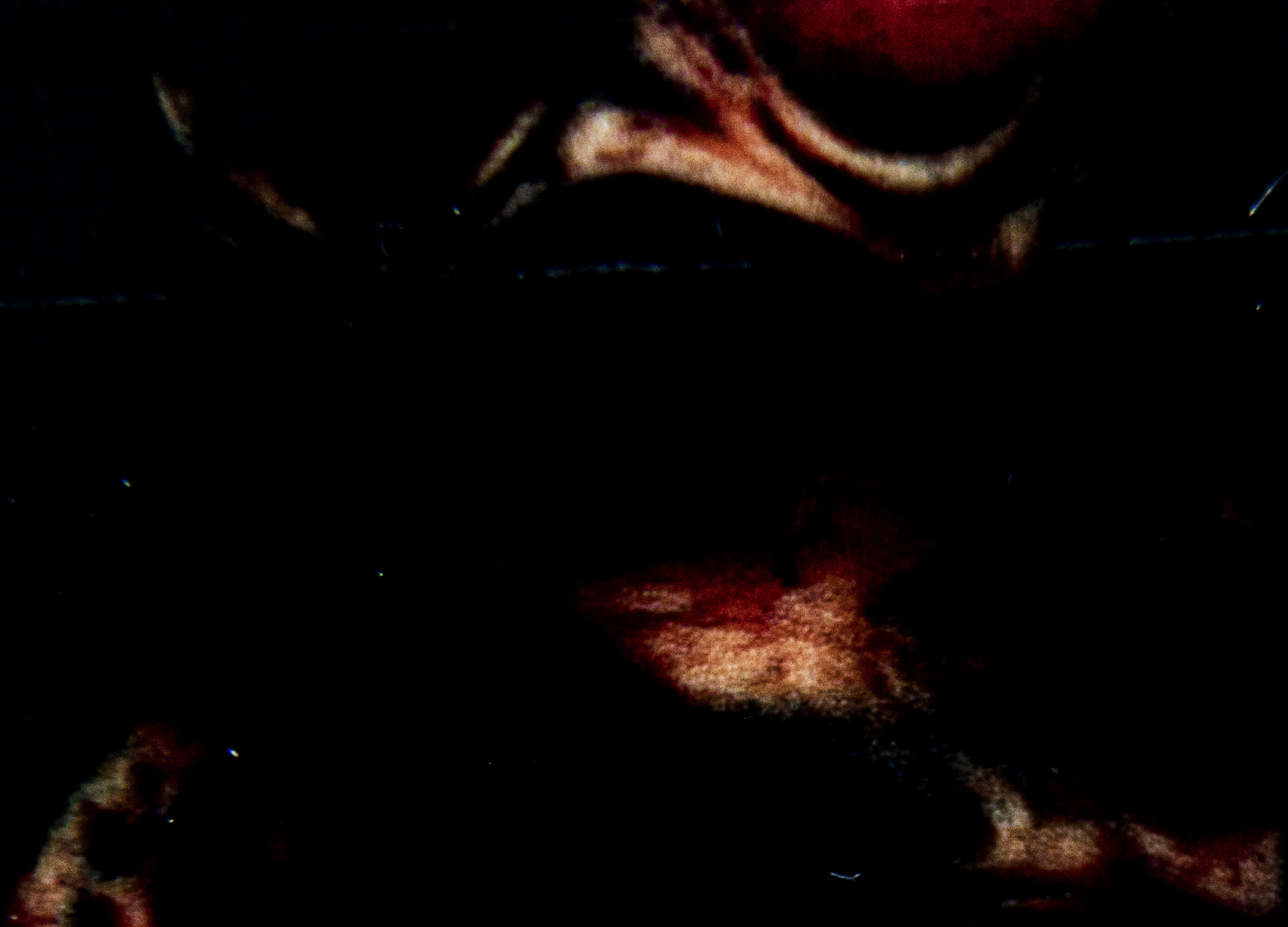

$y^{2}=$

3

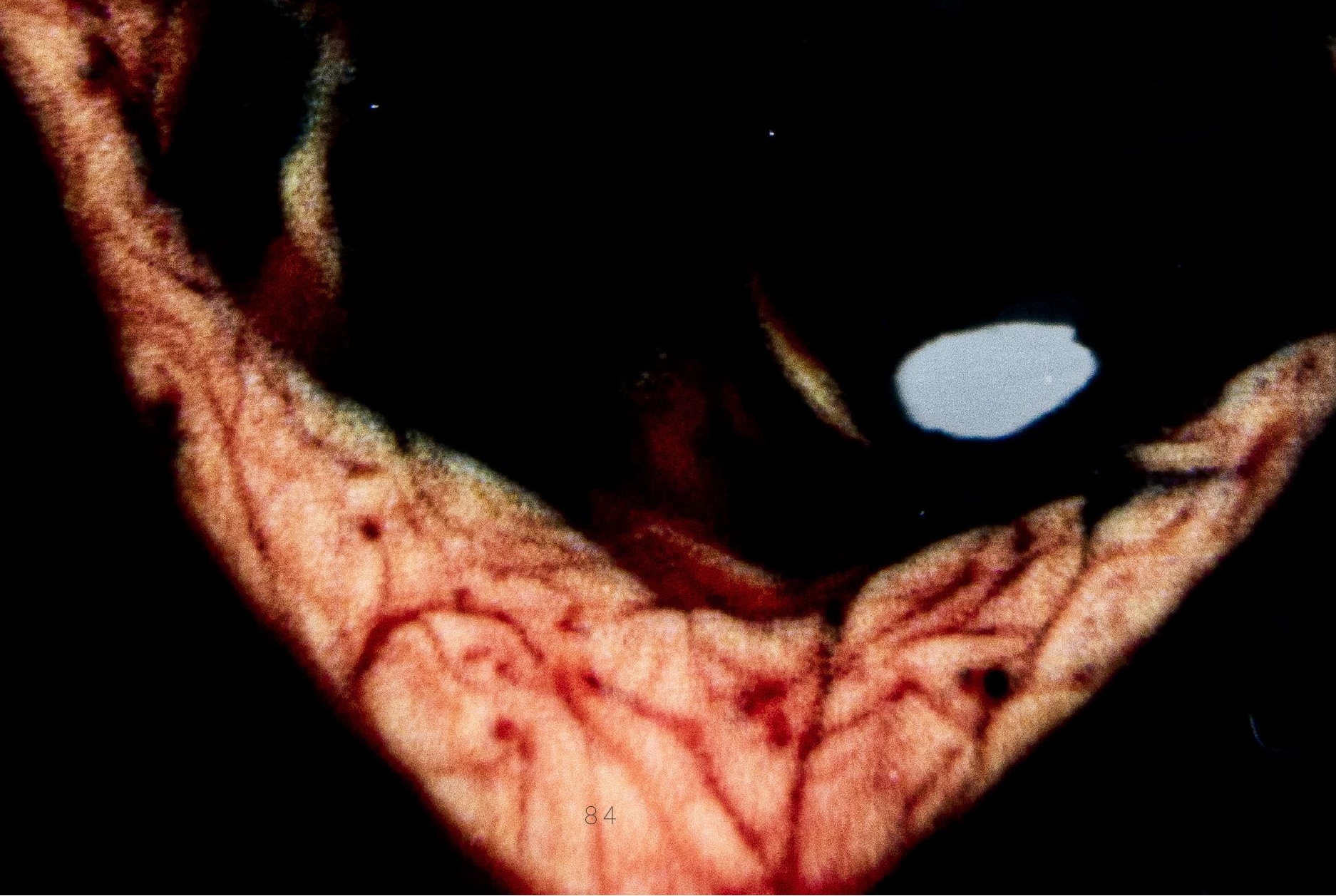


Design Experimentation

Note: All objects that are 3D-printed on the Stratasys J750 ${ }^{\mathrm{TM}}$ 3D-Printer are coated in support material (SUP706 ${ }^{\mathrm{TM}}$ ). SUP706 ${ }^{\mathrm{TM}}$ coats and fills in any layers so that the 3D-printed model can build without failure. This material can be removed and the model post-processed. Typically, for Vero ${ }^{\mathrm{TM}} 3 \mathrm{D}$-prints (hard), it is common to sand and polish the 3D-prints to create a clear finish. The 3Dprints are sanded starting with coarse (100 grit) working up to fine (1500 grit) wet-and-dry sandpaper and then are usually polished with a metal or plastic polish (available at most home hardware stores).

Left:

Figure 39. A bitmap-based 3D-printed section of the Visible Female® heart. 

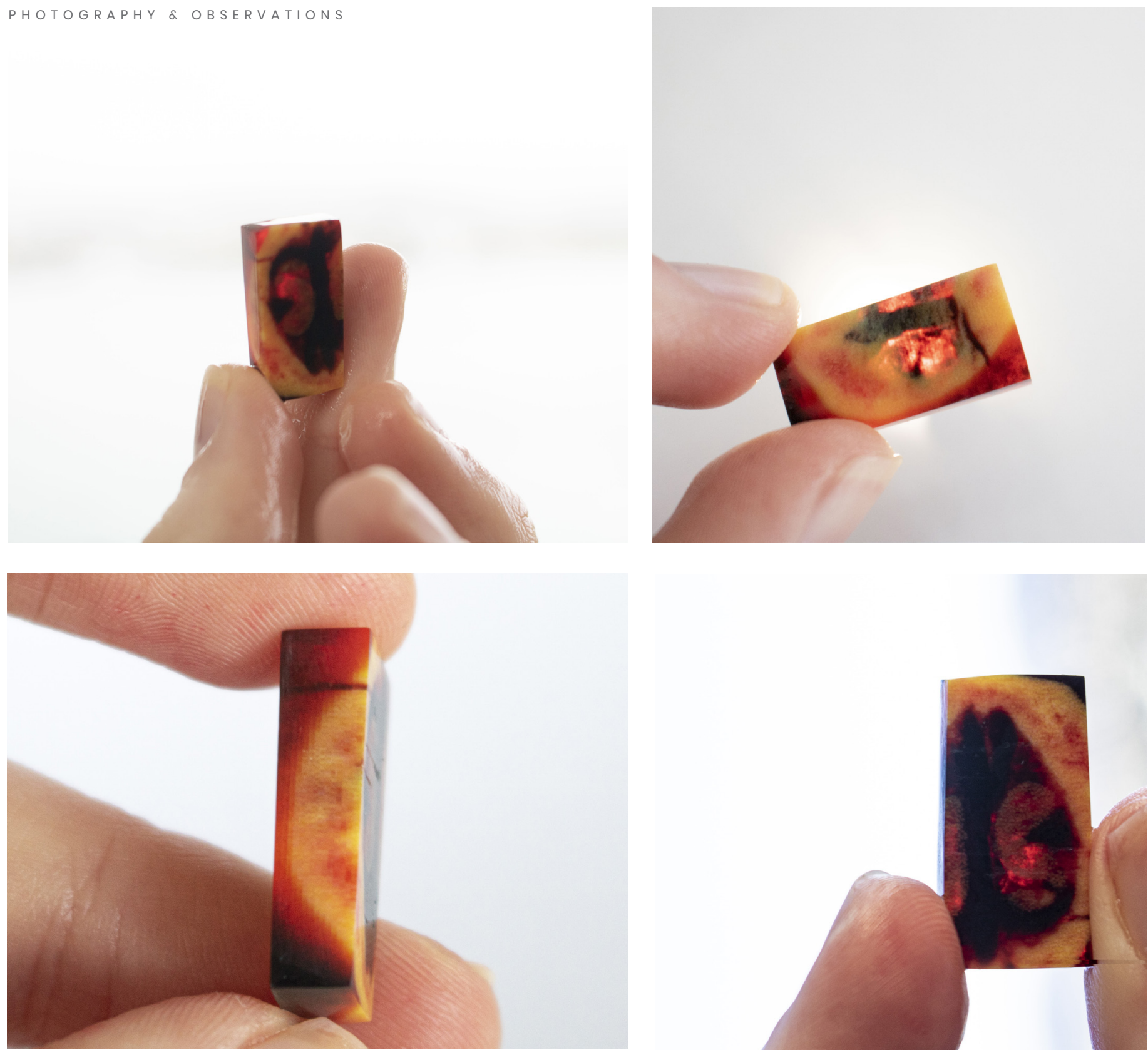

Figure 40. Images of 3D-print \#1 (Visible Female ${ }^{\circledR}$ skull and brain).

To 3D-print a section of the skull and brain to see the transition between two diverse areas of anatomy.
Discovery

The resulting 3D-print drew interest because, when held against a light-source, a detailed structure within the brain is visible. 

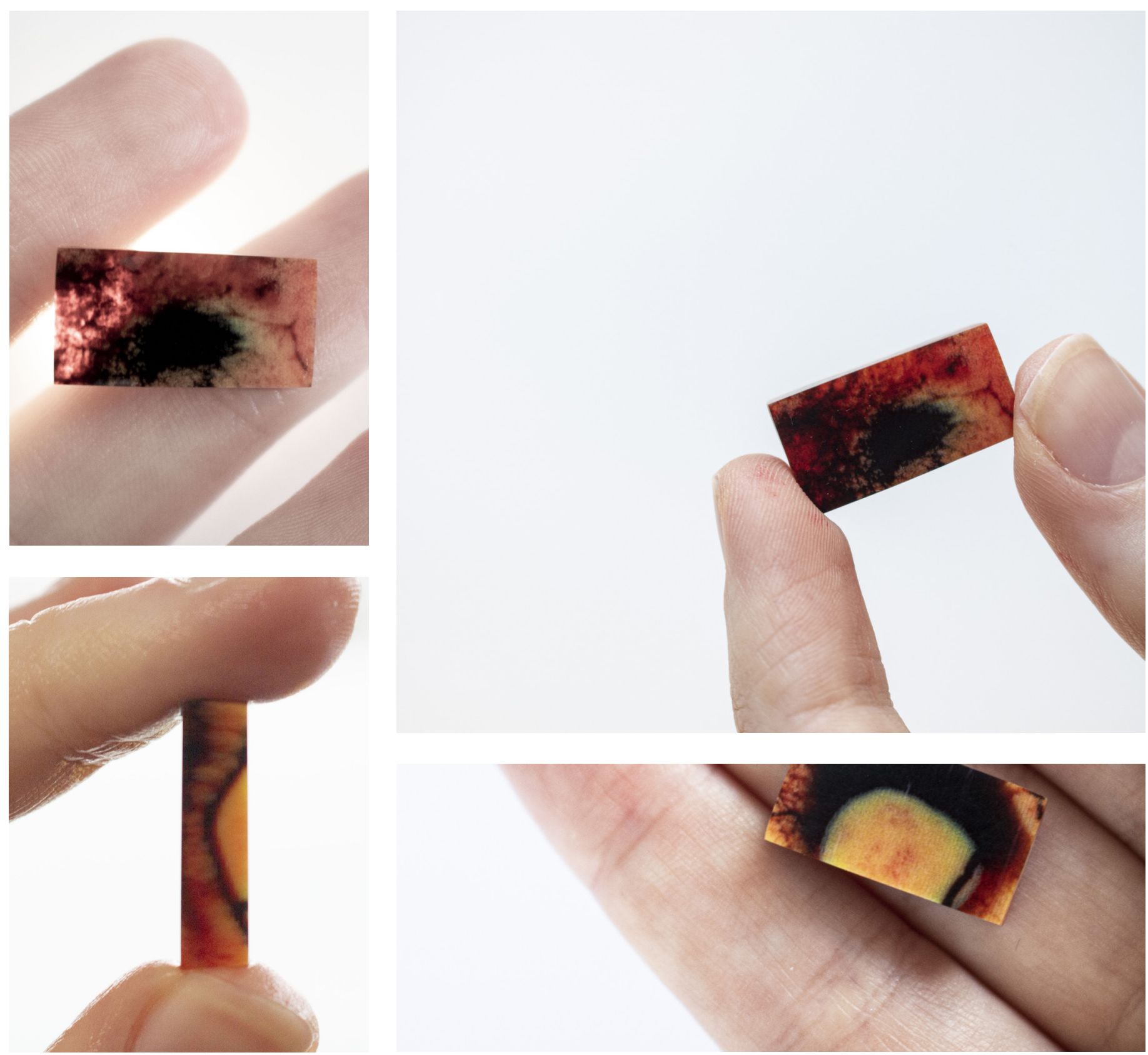

Figure 41. Images of 3D-print \#2 (Visible Female ${ }^{\circledR}$ scalp and skull).

To 3D-print the top of the head, including the skin and the top of the scalp.
The resulting 3D-print was interesting, especially the skin area. The appearance of the 3D-print looked like some form of bruising and the blood vessels are visible. The area between the skull and skin is textured (potentially skin or hair follicles). 

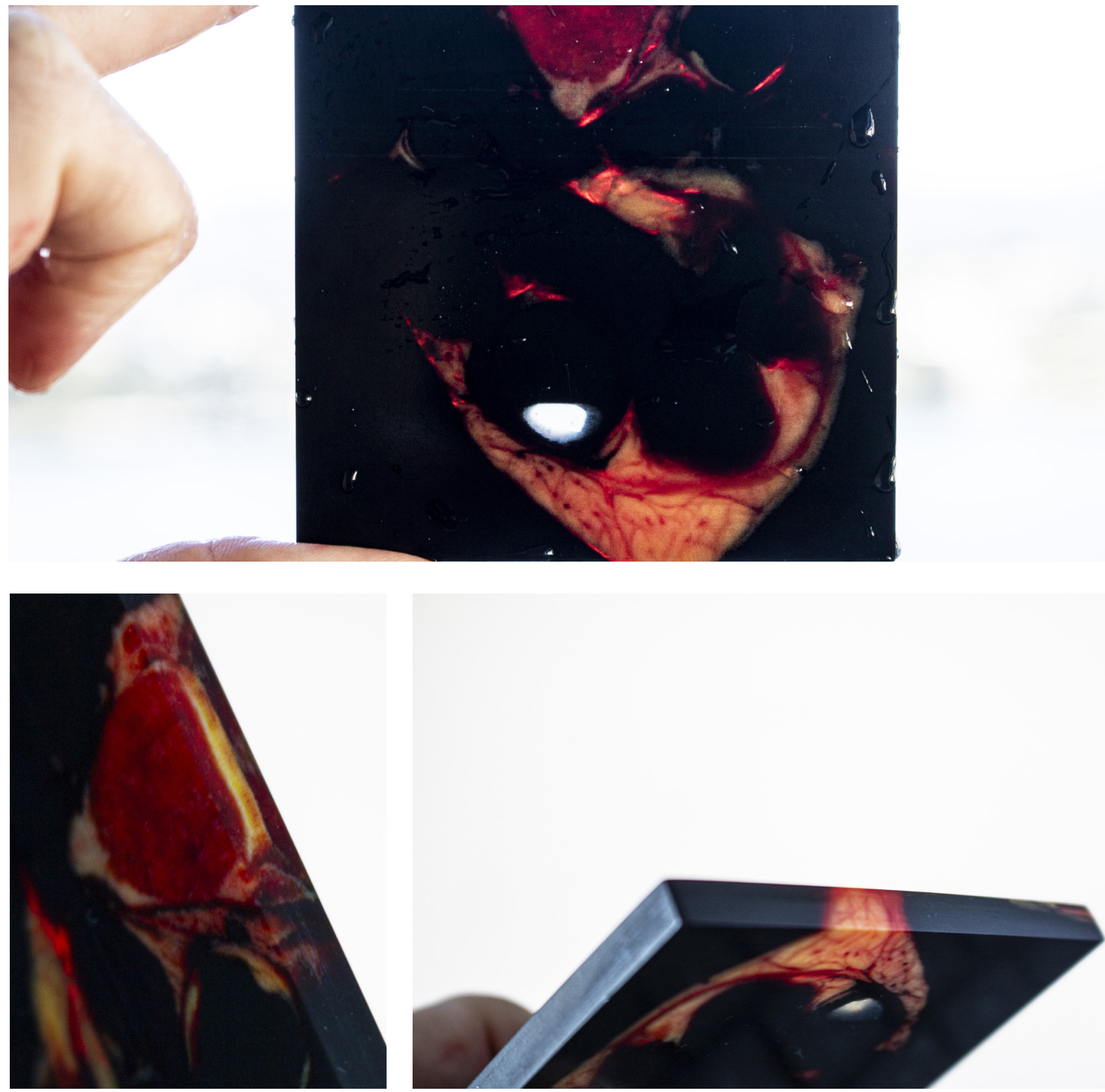

Figure 42. Images of 3D-print \#3 (Visible Female ${ }^{\circledR}$ heart).

\# 3 / Heart Cross-section

A i m Scale 1:2

To 3D-print a complete slice through the heart in order to see the full details \& visibility of structures within the heart/lungs.
Structures within the fat - "channels" - for blood to run through appeared vividly. With this said, other areas (such as the lungs surrounding the heart) appeared black with no observable detail. 

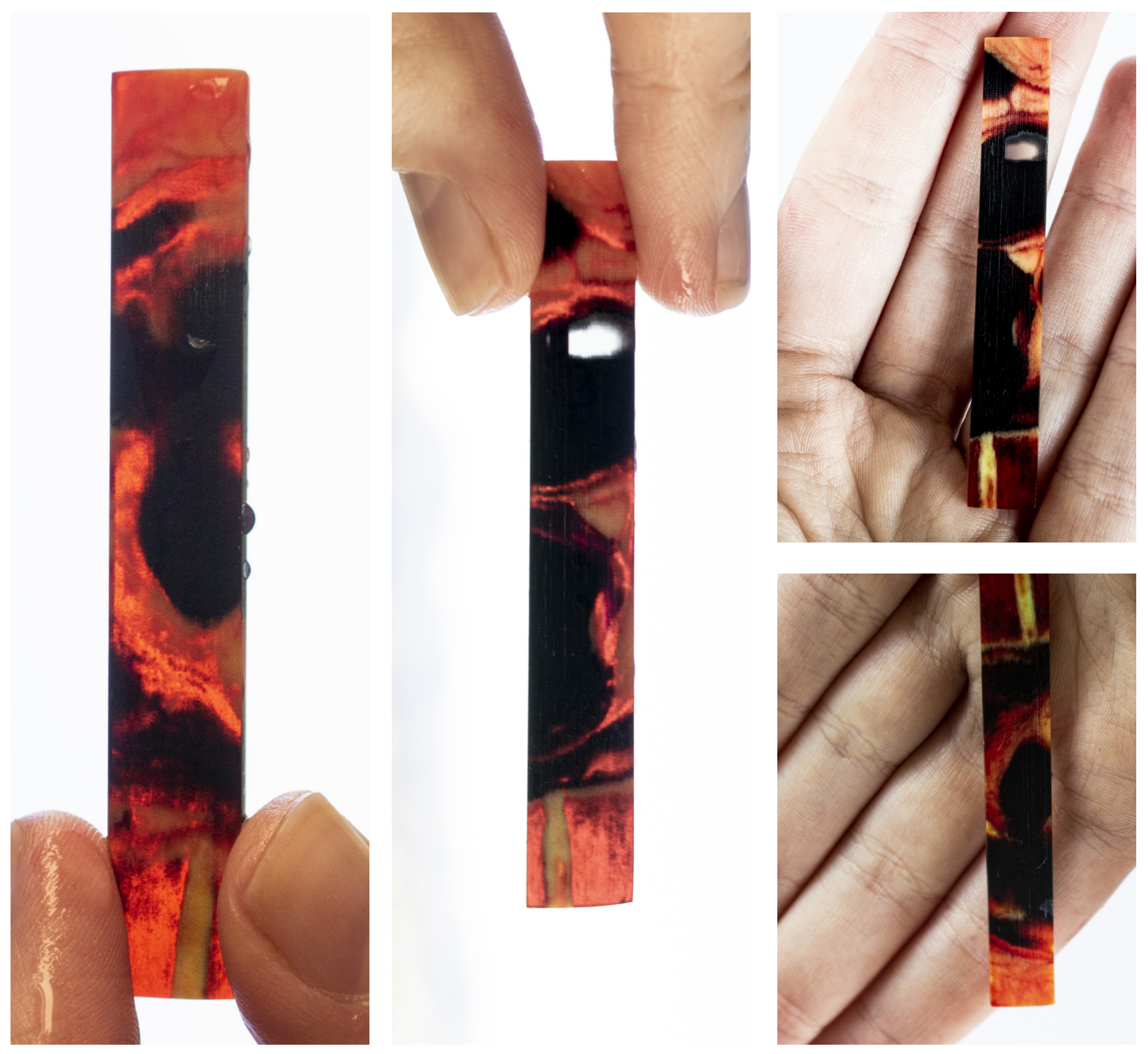

Figure 43. Images of 3D-print \#4 (Visible Female ${ }^{\circledR}$ heart).

\# 4 / Heart Cross-sections (thin slices)

To 3D-print slices through different areas of the heart to understand 1:1 details.
These 3D-prints were interesting between they showed a 1:1 representation of heart structures, The detail captured was of highfidelity, but also dark which meant some parts of the 3D-prints were completely blacked out. 

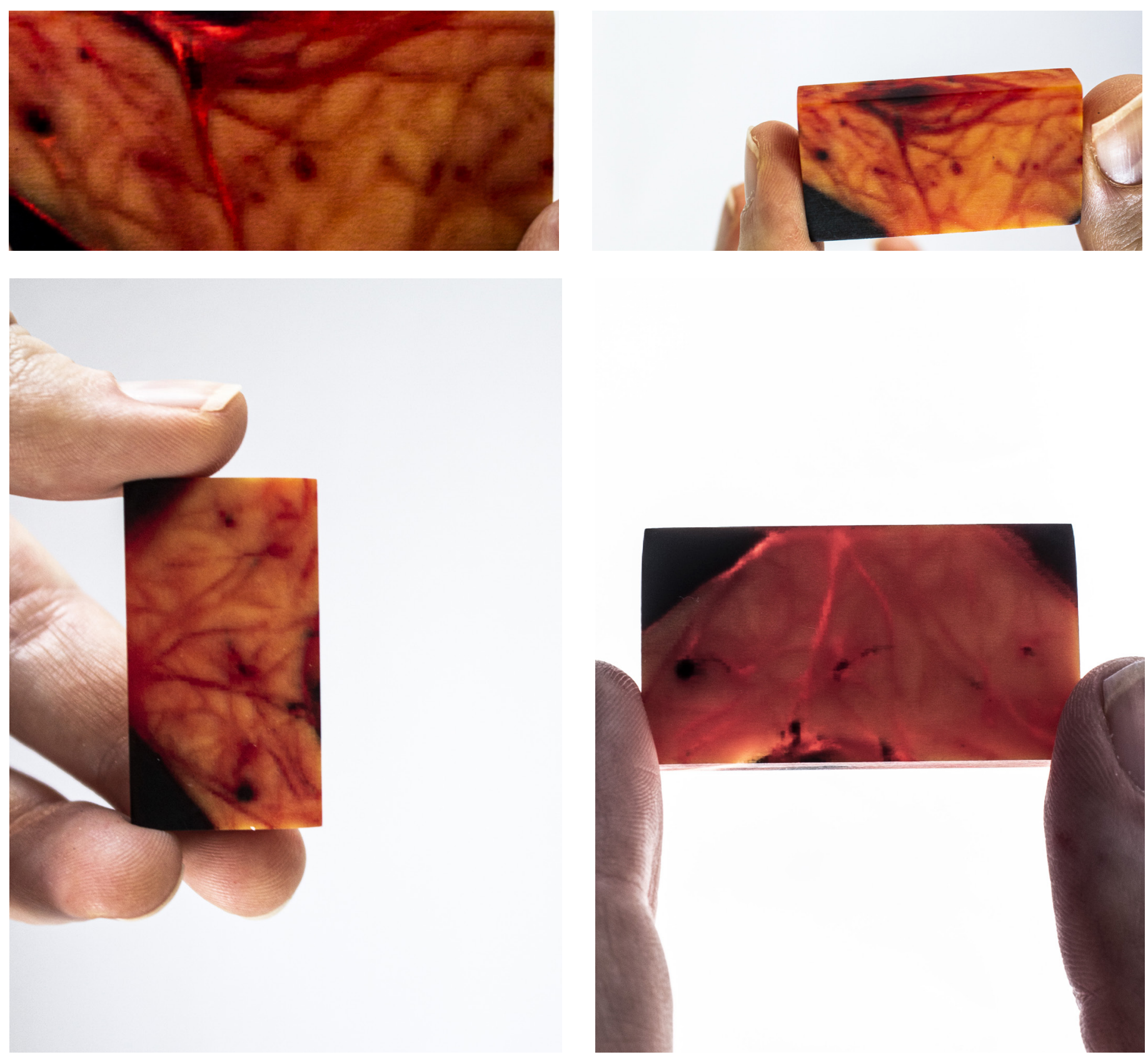

Figure 44. Images of 3D-print \#5 (Visible Female ${ }^{\circledR}$ fat).

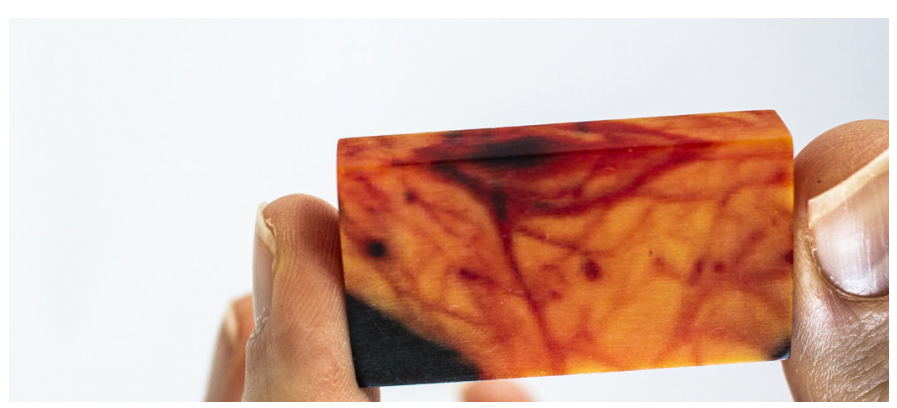

A i m

To 3D-print an area of 1:1 fat surrounding the heart. Since fat was executed well in 3Dprint \#3, this 3D-print aimed to look at the details further.

Scale 1:1

\# $5 / \mathrm{Heart} F a t$

T Bran Because of the increase in scale, more VeroMa-
genta-V $V^{\mathrm{TM}}$ is spread through VeroPureWhite
which means that the fat appears more translucent and less white and opaque. 

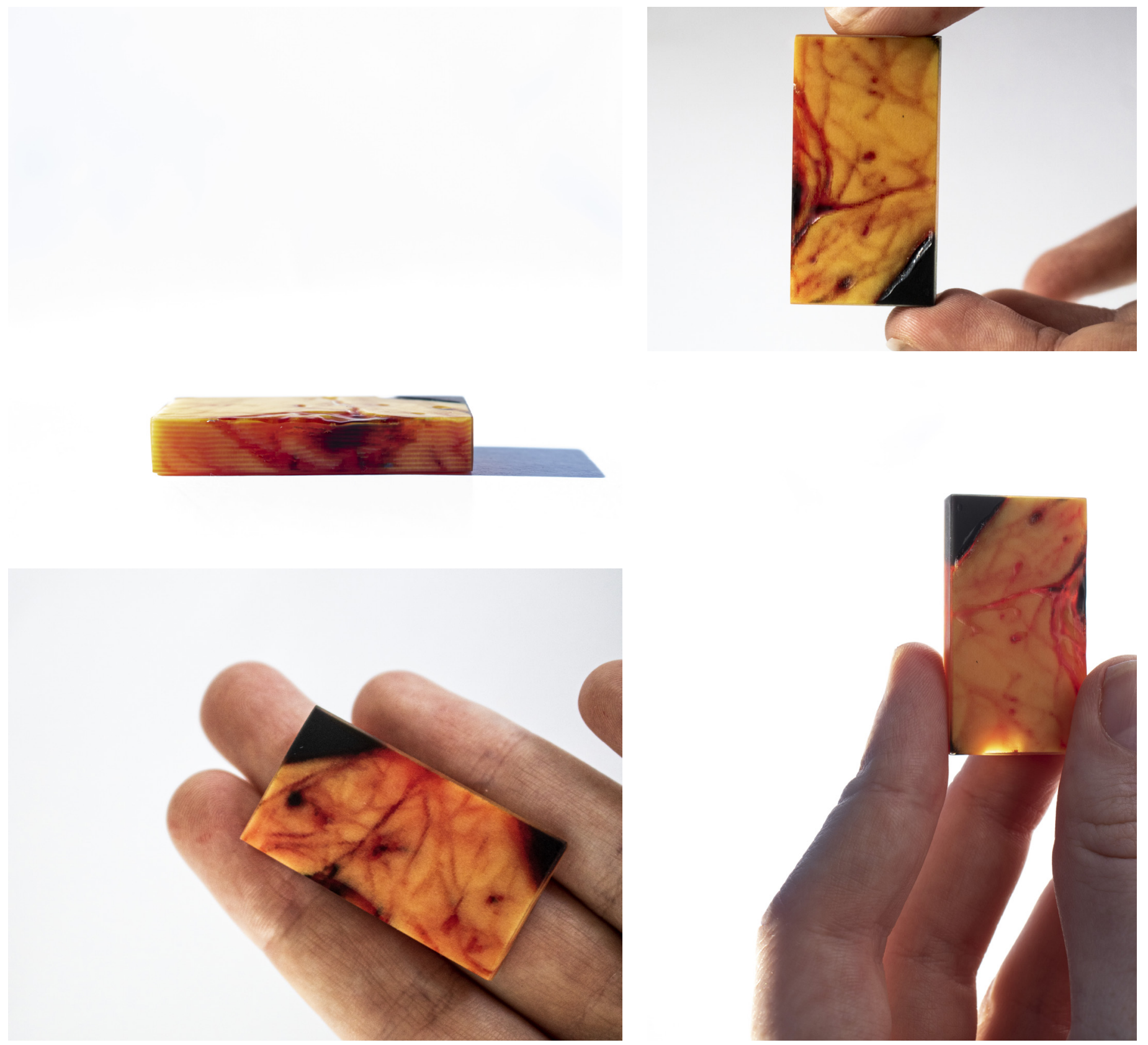

Figure 45. Images of 3D-print \#6 (Visible Female ${ }^{\circledR}$ fat with embedded support material).

\#6/ HeartFat (with embedded Sup706 тм)

A i $m$

To 3D-print the same file as 3D-print \#5, but replacing the red or "blood" in every other layer with support structure, SUP706 ${ }^{\mathrm{TM}}$. The aim of the was to see if the resulting $3 \mathrm{D}$-print produced micro "channels" that "blood" could be injected into in the post-processing stages.
The resulting 3D-print was not the expected result but created an interesting texture on one side of the model. Instead of creating micro "channels", the 3D-printed structure collapsed (producing texture). 


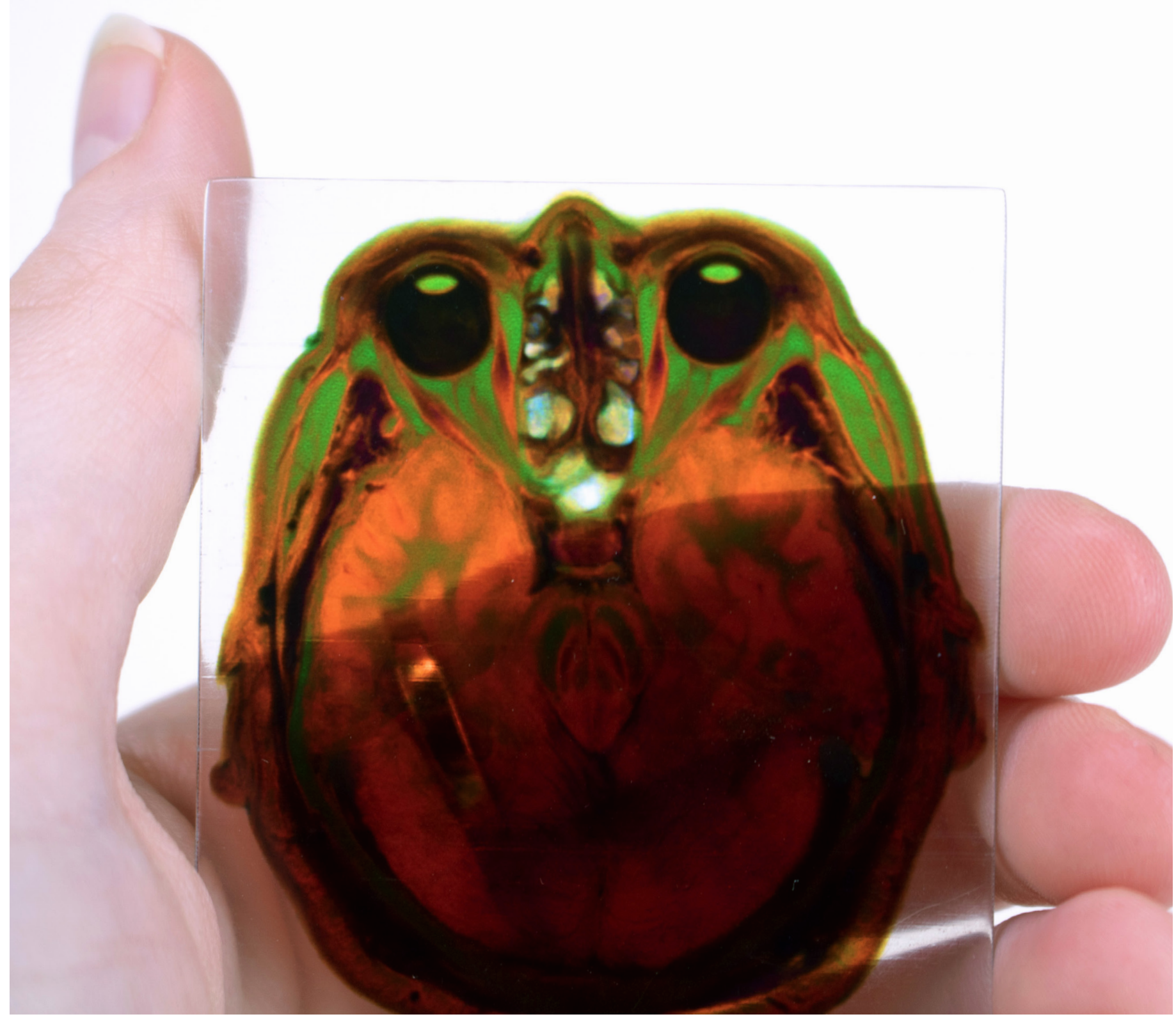

Figure 46. Image of 3D-print \#7 (Visible Female ${ }^{\circledR}$ brain slice).

To take a small slice through the brain (including areas such as the sinuses) to see and understand the appearance of the brain. This 3D-print utilized the full material range, including VeroClear ${ }^{\mathrm{TM}}$ within the brain structure.
The inclusion of VeroClear ${ }^{\mathrm{TM}}$ within the brain structure made the 3D-print dark. When the print is held up to a light-source, the VeroClear ${ }^{\mathrm{TM}}$ caused some parts of the model to appear bright green. 


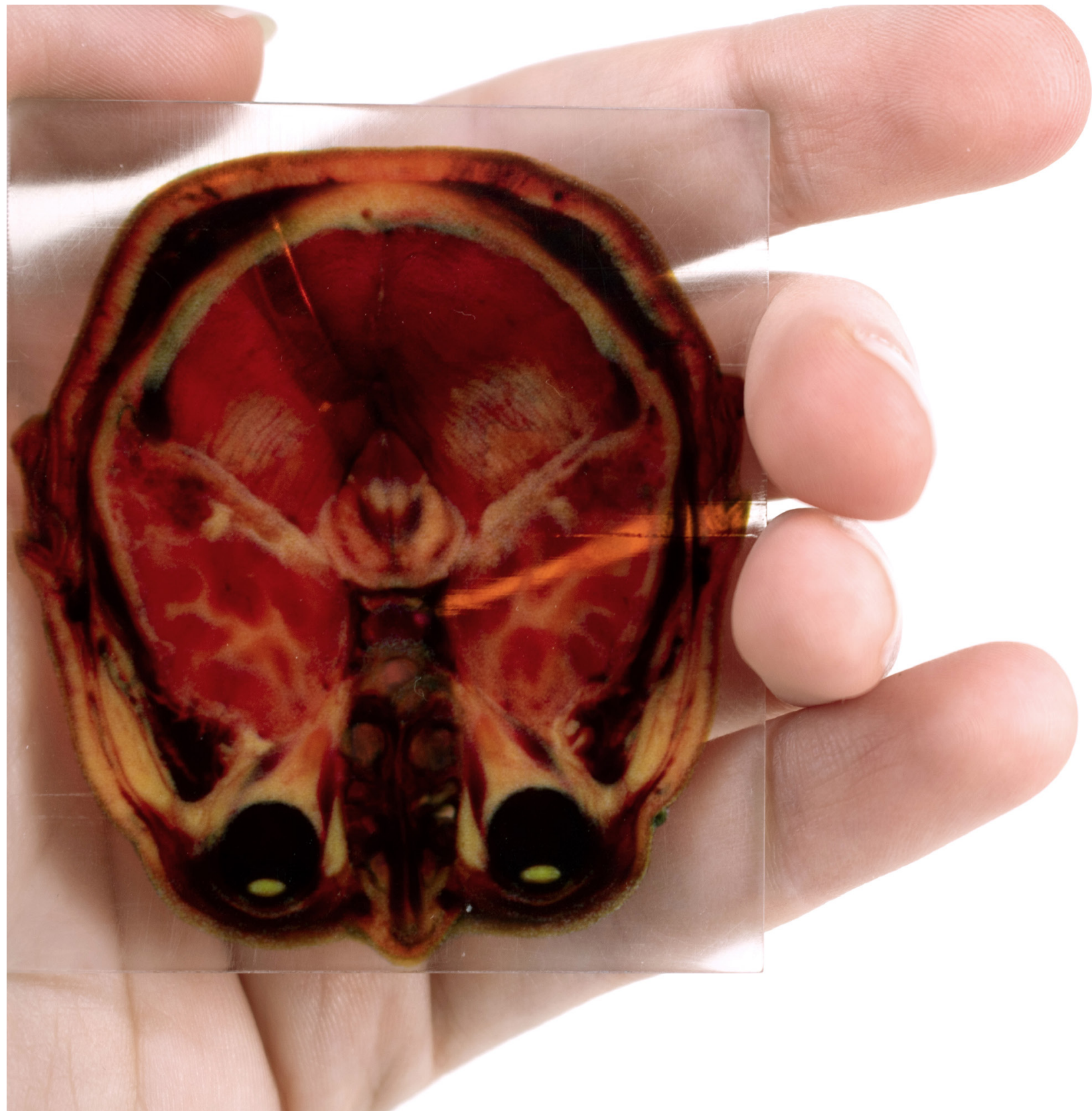

Figure 47. Image of 3D-print \#8 (Visible Female ${ }^{\circledR}$ brain slice without clear material).

\# 8 / Brain Slice (without Veroclear ${ }^{\mathrm{TM}}$ )

A i m

To take a small slice through the brain (including areas such as the sinuses) to see and understand the appearance of the brain. The file was the same as 3D-print \#7. This 3D-print utilised the full material range including VeroClear ${ }^{\mathrm{TM}}$. However, VeroClear ${ }^{\mathrm{TM}}$ was only used for the bounding box and was not used within the brain structure.
Avoiding the use of VeroClear ${ }^{\mathrm{TM}}$ within the brain structure resulted in a 3D-print with colour that was more representative of cadaveric anatomy, mimicking anatomical colour much more realistically. 

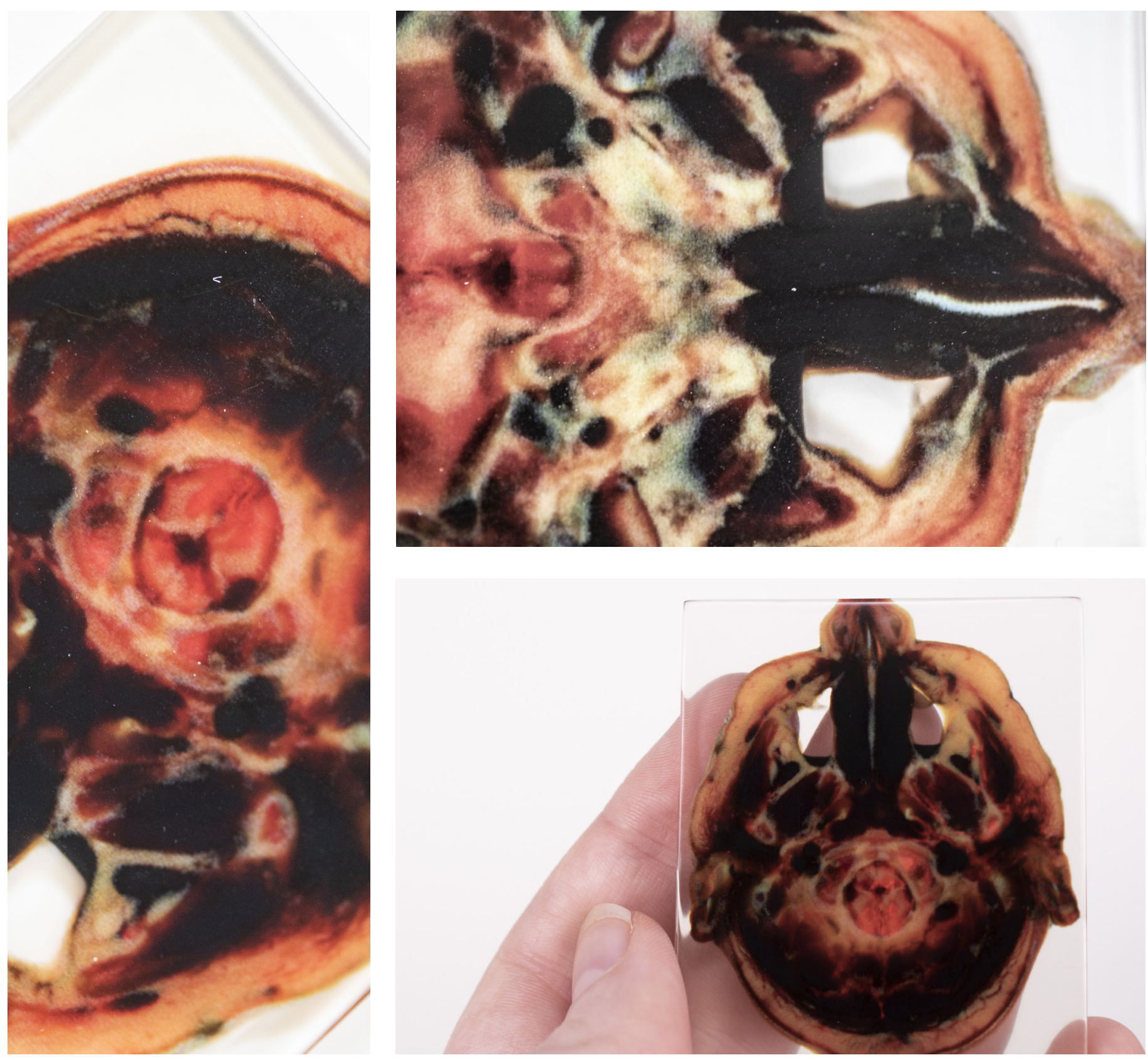

Figure 48. Images of 3D-print \#9 (Visible Female ${ }^{\circledR}$ brain slice).

To take the same file as 3D-print \#8, but add more layers to create a thicker 3D-print with more detail and depth.
The resulting 3D-print was detail, clear, \& realistic - showing elements of anatomy such as the nose and ears. 


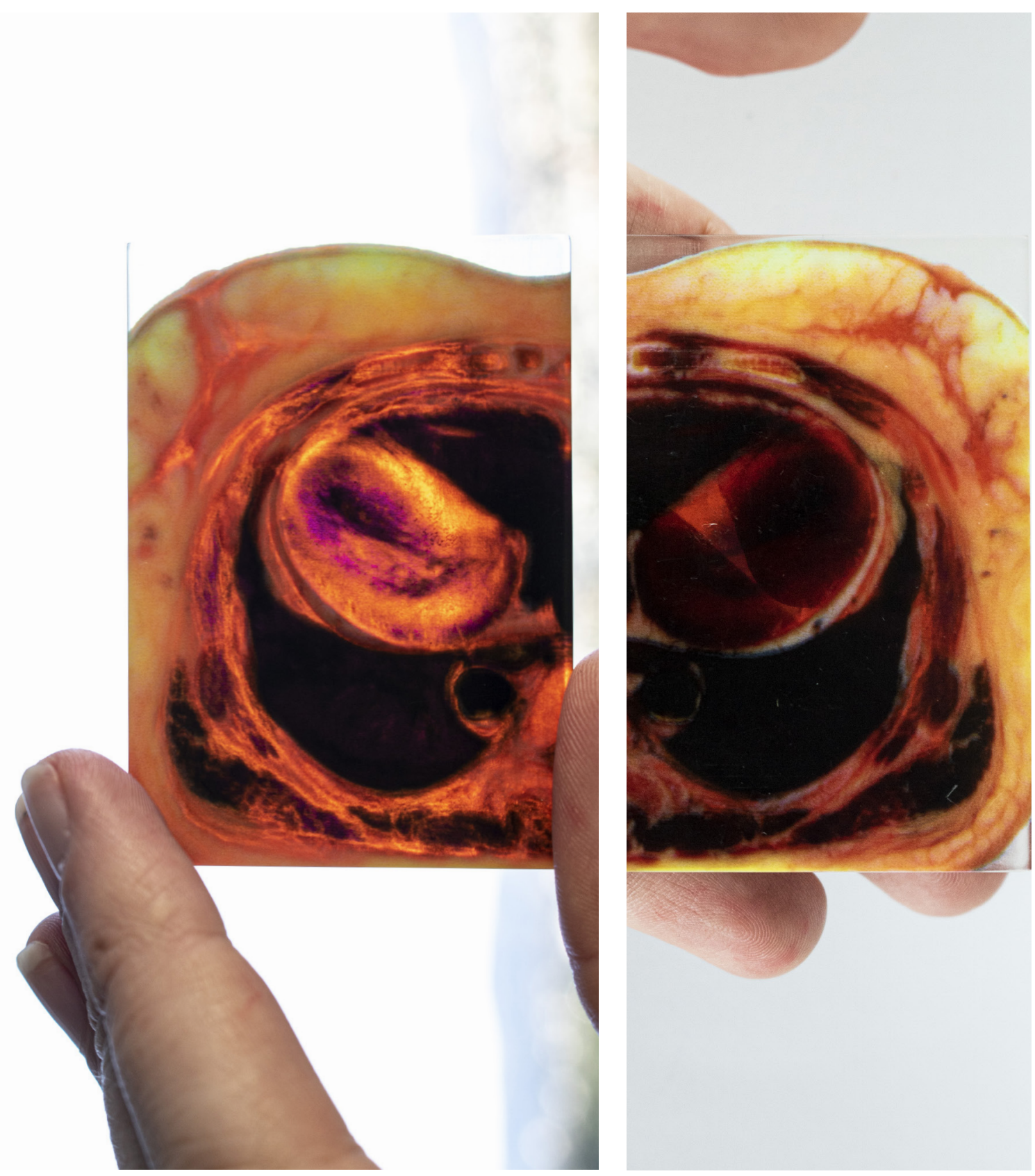

Figure 49. Images of 3D-print \#10 (Visible Female ${ }^{\circledR}$ breast).

To 3D-print a slice through the breast (including the heart, ribs and breast tissue) to see the intricacies in the breast such as fat, lobules and ducts.
Details such as fat, ribs (including the internal structure), structures within the breast and the nipple were all captured within the resulting 3D-print. 

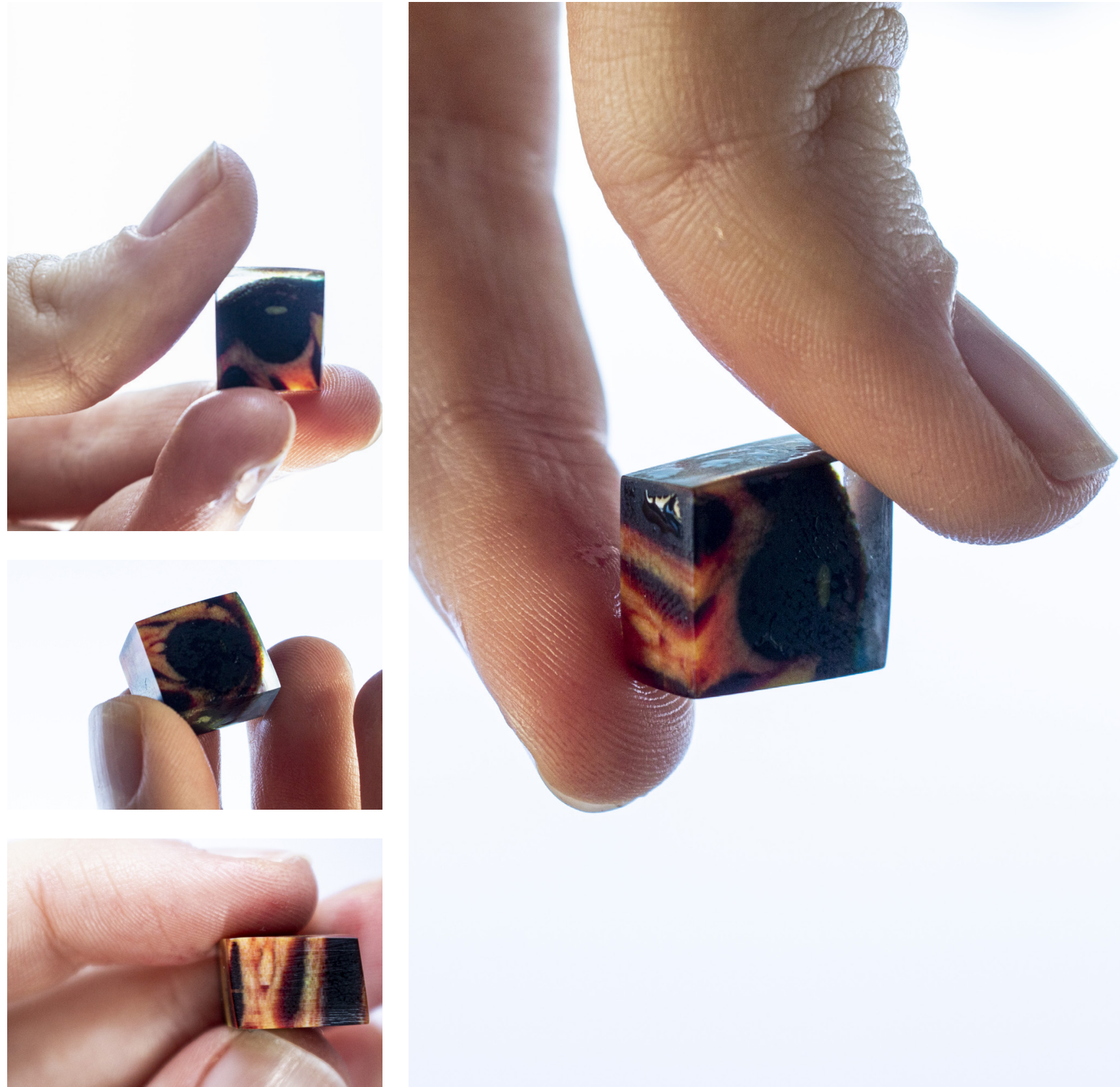

Figure 50. Images of 3D-print \#11 (Visible Female ${ }^{\circledR}$ eyeball).

To take a small section of the eyeball to see if the colours and translucency of the eyeball could be replicated.
Discovery

The eyeball appeared dark and black, but the muscle structures surrounding the eyeball were highly detailed and realistic. The scale was meant to be $1: 1$, but for some reason appeared much smaller, which meant that there was a glitch in the filepreparation method. 

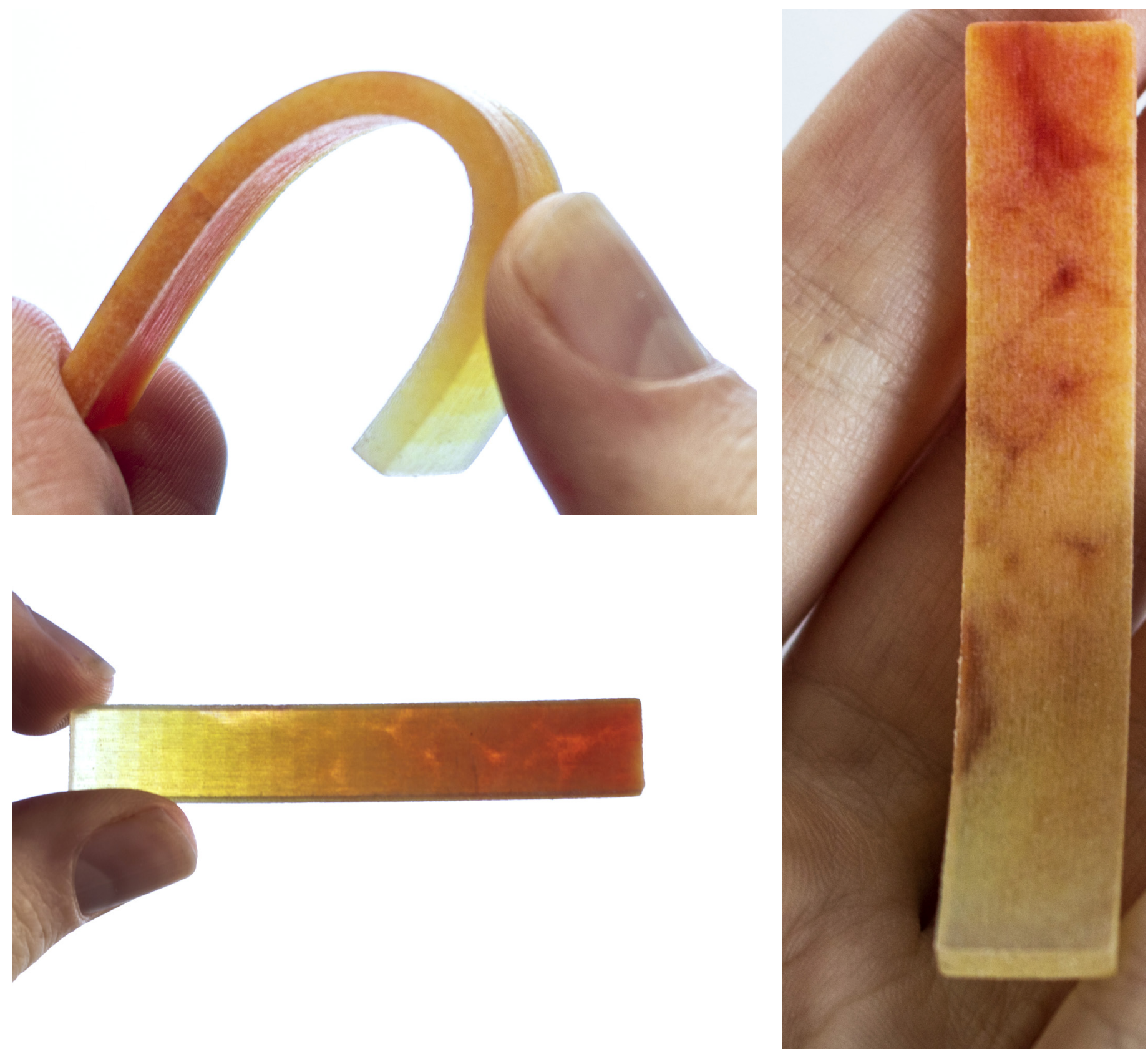

Figure 51. Images of 3D-print \#12 (Visible Female ${ }^{\circledR}$ bendy fat).

\#12/ Bendy Fat

A i m

To 3D-print a slice of fat with Agilus $30^{\mathrm{TM}}$ dispersed through the 3D-print to create some form of movement whilst preserving anatomical colour - laying the foundation for Case Study 2.
Scale 1:1

Dispersing Agilus30 $0^{\mathrm{TM}}$ through the fat meant that the 3D-print moved (had dynamics). Although some anatomical colour was preserved, a lot was lost and the $3 \mathrm{D}$ print did not visually mimic anatomy in the same way as the Vero ${ }^{\text {TM }} 3 \mathrm{D}$-prints. 
The parallel prototyping in Case Study 1 mimicked anatomical colour realistically. Therefore, moving forward, the decision was made to 3D-print the half of Visible Female ${ }^{\circledR}$ from head to toe at a 1:12 scale. The 1:12 scale was decided upon because it was a way to show the capabilities of the Stratasys J750 ${ }^{\mathrm{TM}} 3 \mathrm{D}-$ Printer without sacrificing logistics such as easy transportation and sustainable use of materials.

Figure 52. Image of full Visible Female ${ }^{\circledR}$ bitmap-based 3D-print (final model).

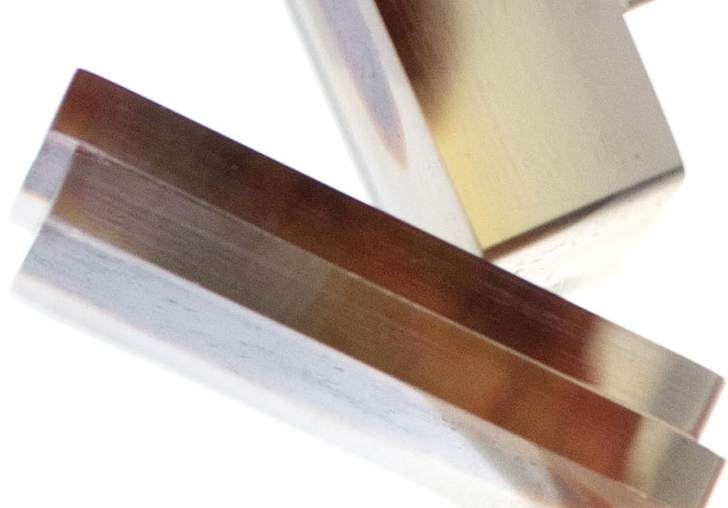


Fina I Design 

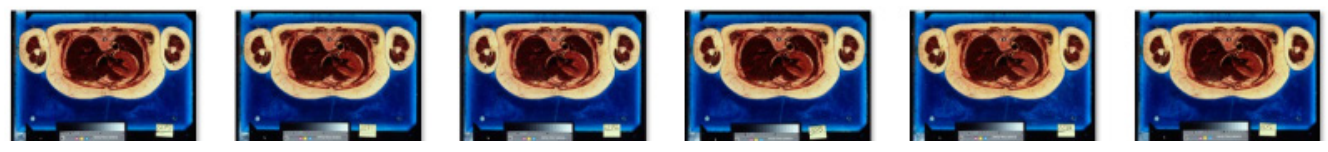

Figure 53. Visible Female ${ }^{\circledR}$ in blue embedding media.
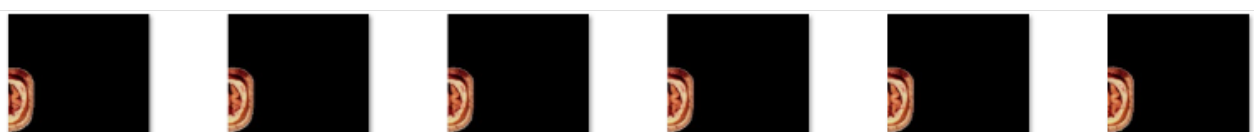

Figure 54. Visible Female ${ }^{\circledR}$ sections with blue embedding media removed.
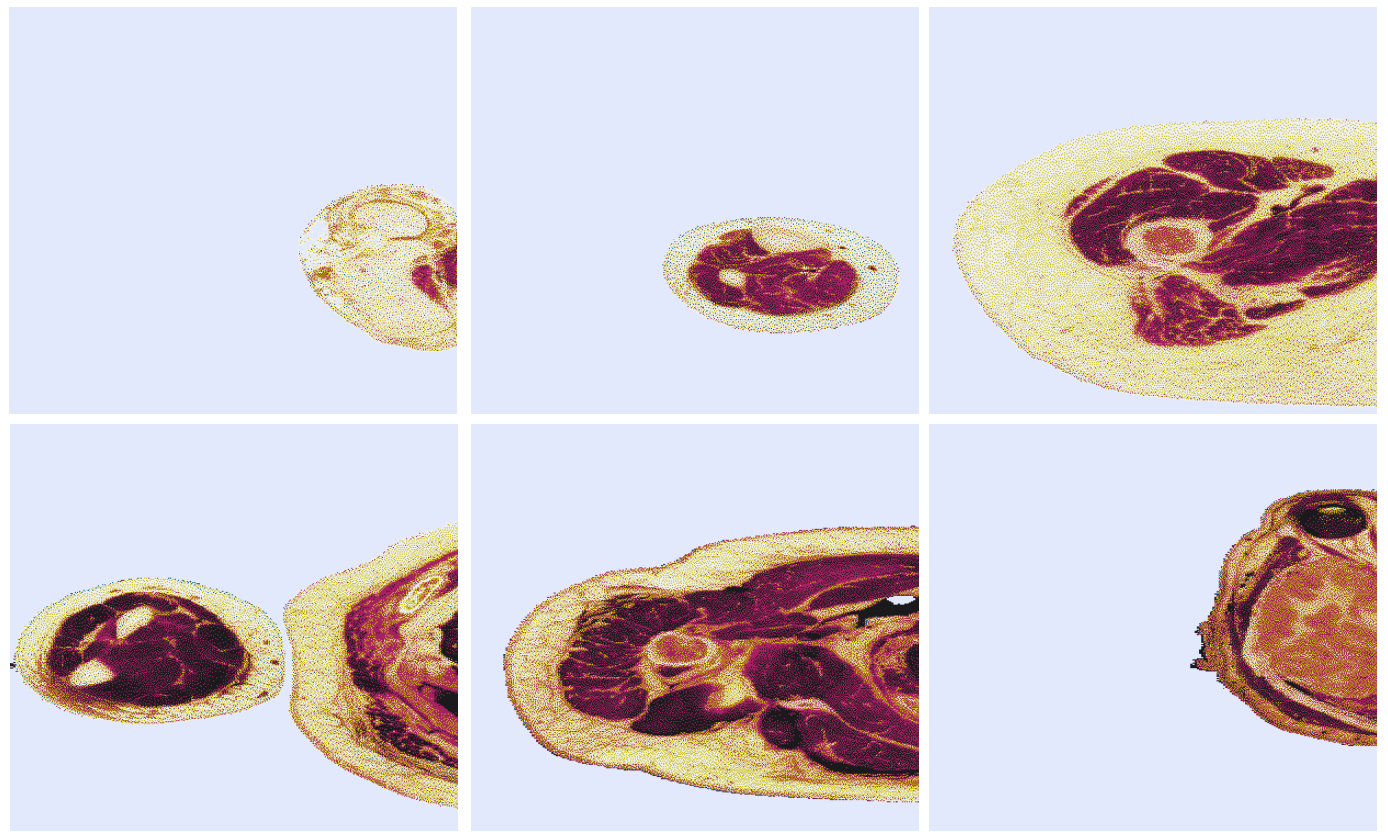

Figure 55. Digitally halftoned Visible Female ${ }^{\circledR}$ bitmap images.

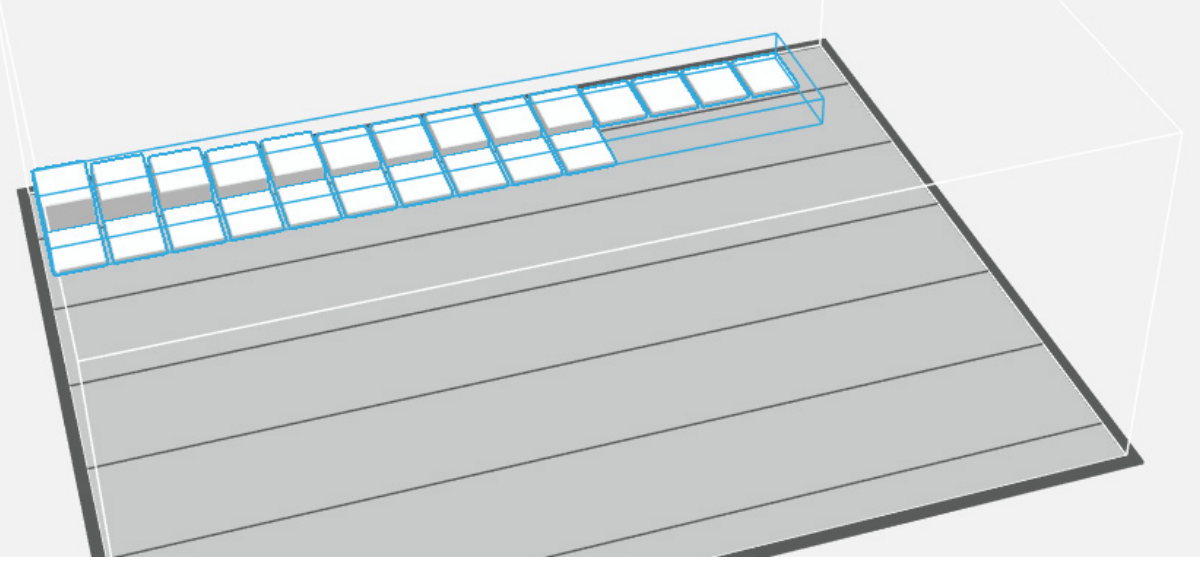

Figure 56. GrabCAD Print tray with the full 1:12 Visible Female ${ }^{\circledR}$ files. 
Using the same workflow presented in the Design Exploration research phase, the Visible Female ${ }^{\circledR}$ bitmap images were prepared for 3D-printing. Originally, the female cadaver was embedded and frozen in an embedding media made of a 3\% gelatine solution and blue dye (Westwood, 2002, p. 231). Therefore, a colour threshold was used to eliminate the blue embedding solution (Figure 53). The images were cropped down the centreline of the body and a boundary box was placed around the Visible Female (the black box seen in Figure 54).

The masked datasets were scaled into the Stratasys J750 ${ }^{\mathrm{TM}}$ 3D-Printer's native $\mathrm{X}$ and $\mathrm{Y}$ resolutions and material dithering algorithms (digital halftoning) were used to create pixel combinations using the primary 3D-printer materials of VeroPureWhite ${ }^{\mathrm{TM}}$, VeroCyan-V $\mathrm{V}^{\mathrm{TM}}$, VerowMagenta-VTM, VeroYellow-V $\mathrm{V}^{\mathrm{TM}}$ and VeroBlackPlus ${ }^{\mathrm{TM}}$. VeroClear ${ }^{\mathrm{TM}}$ was assigned to the bounding box.

Images were processed through GrabCAD Voxel Print Utility and were exported as a GrabCAD Voxel Files (.GCVF file). A total of 5,102 images were processed to complete the Visible Female ${ }^{\circledR}$ 3D-print. The Visible Female ${ }^{\circledR}$ was split into cross-sectional blocks with different thicknesses for the purpose of this study.

A total of 24 .GCVF files were processed and 3D-printed. The resulting 24 individual 3D-prints were stacked on top of each other to form the full 3D-printed Visible Female ${ }^{\circledR}$, varying in slice thickness (highlighting that bitmap-based printing can produce thin slices or thick blocks). Thick blocks were used to show more detailed areas of anatomy (such as the hand and chest regions) and thinner slices were used to show detailed areas (such as the thigh).

Each pixel in the bitmap images is representative of one voxel being placed on the print tray. 
PHOTOGRAPHY $\&$ OBSERVATIONS

\# 13 / 3 D-Printed Visible Female(Visible Human Project $\left.{ }^{\circ}\right)$

Figure 57-67. Images of the final model of the Visible Female ${ }^{\circledR}$.

The measurements of the $1: 12$ Visible Female ${ }^{\circledR}$ model are $X=28.55 \mathrm{~mm}, \mathrm{Y}=35.65 \mathrm{~mm}, \mathrm{Z}=137.75 \mathrm{~mm}$.

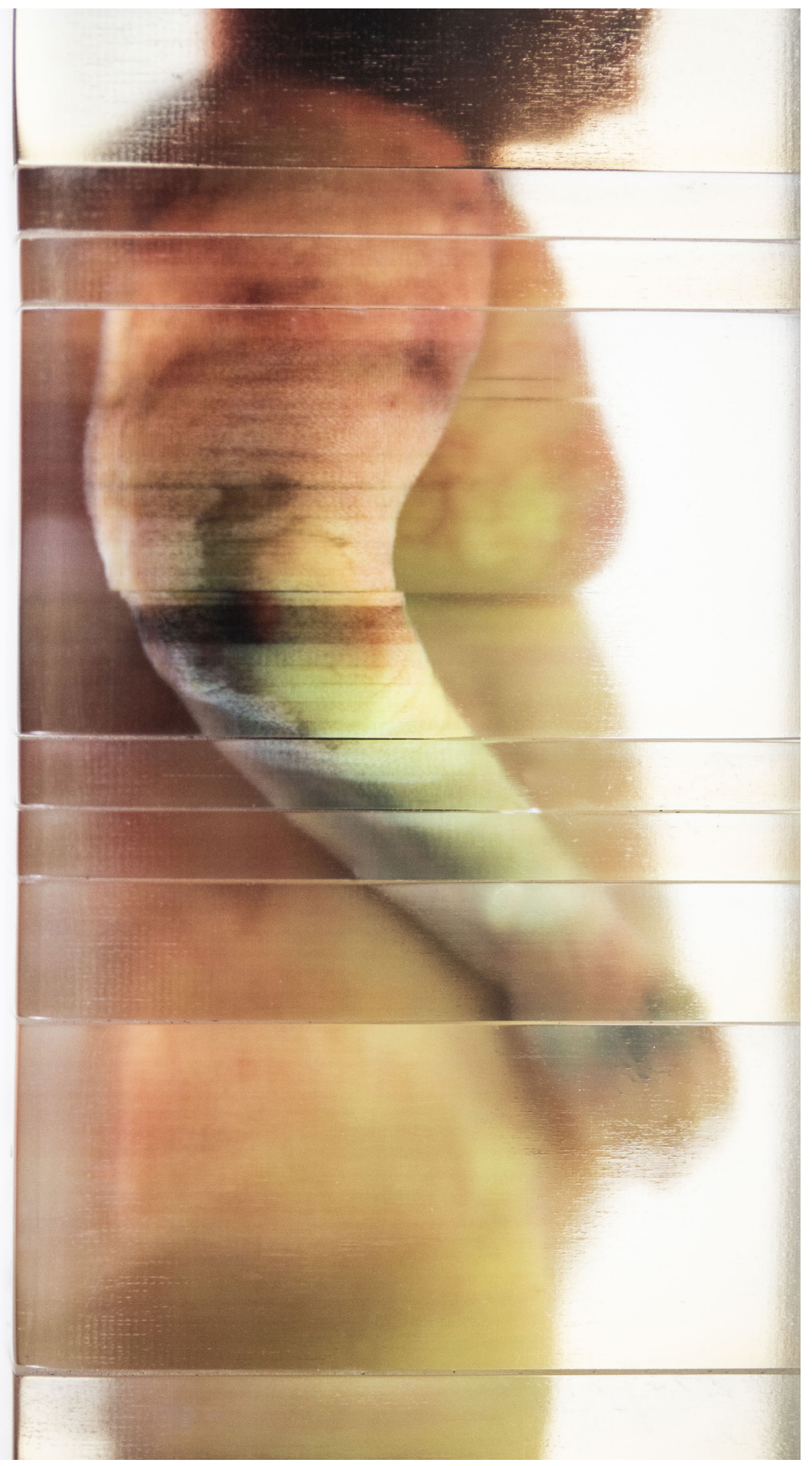




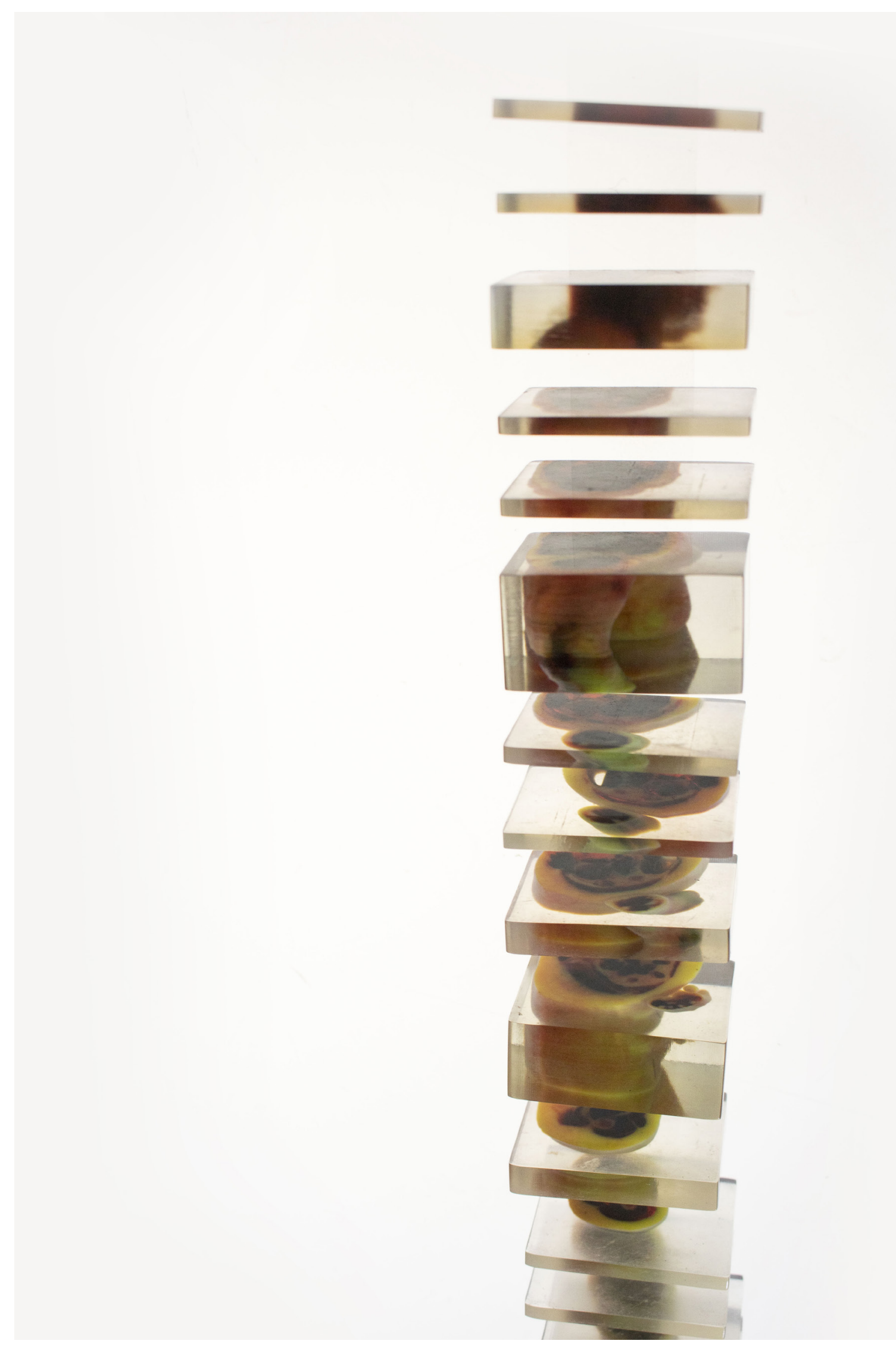




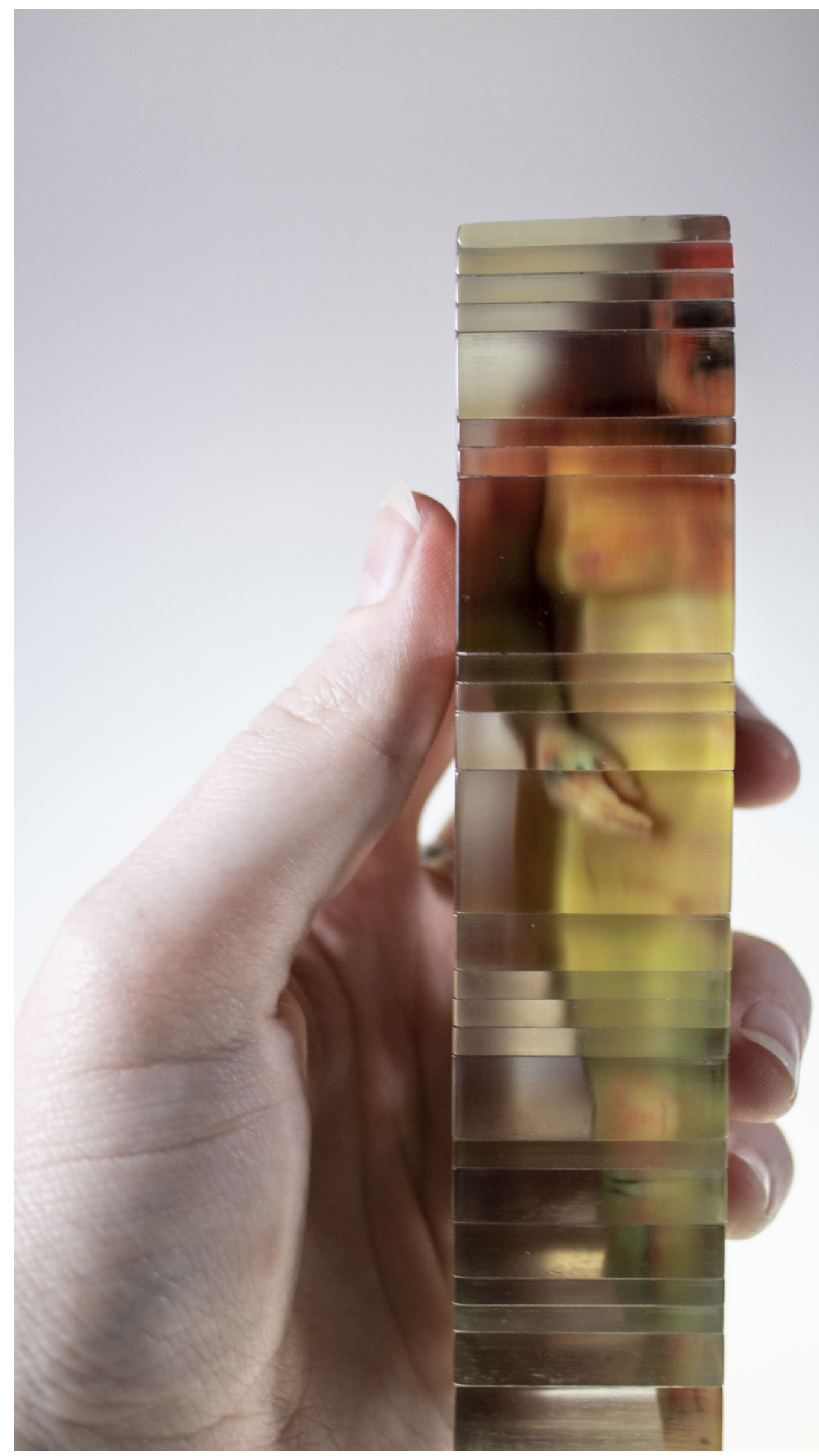




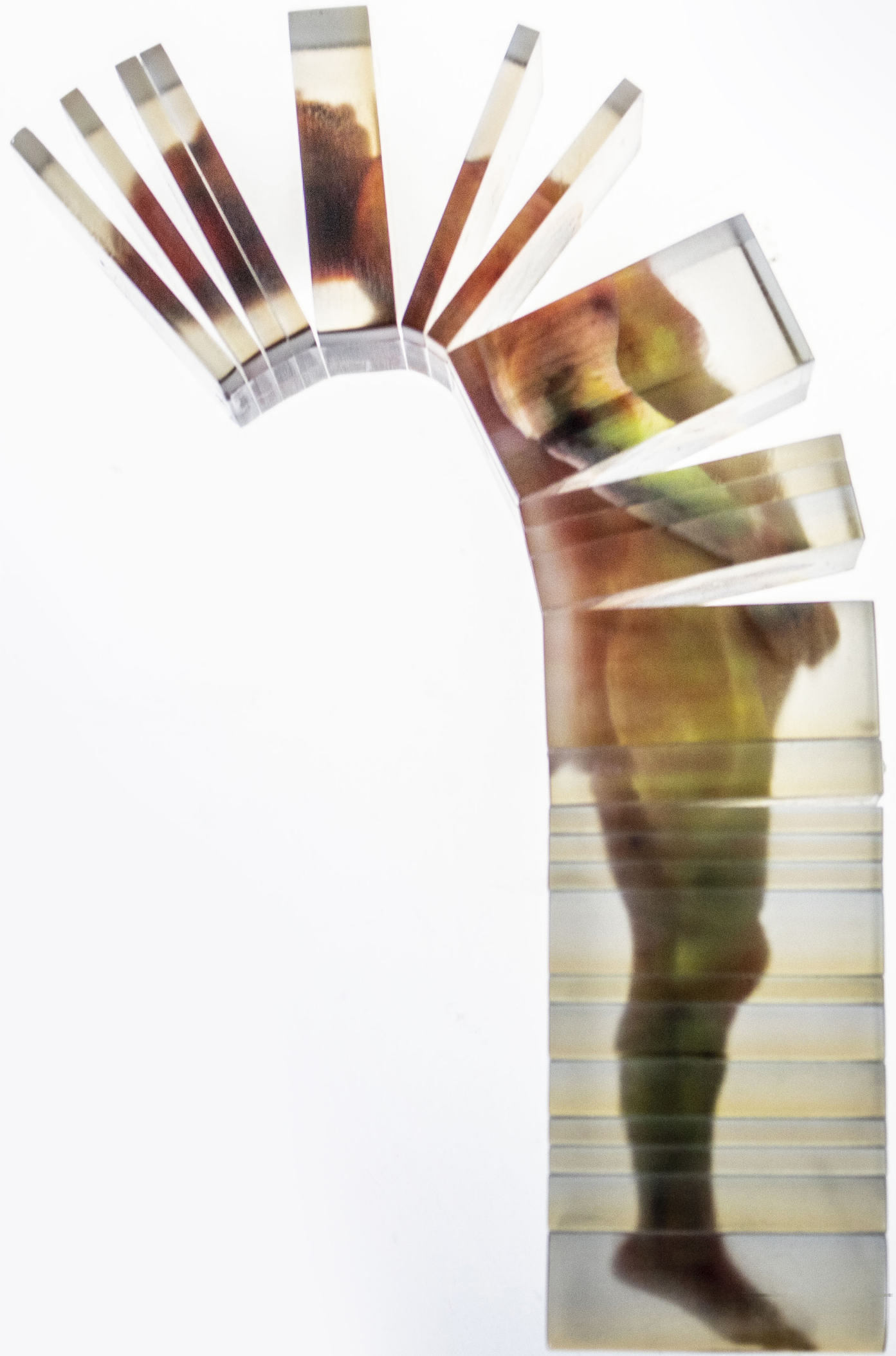




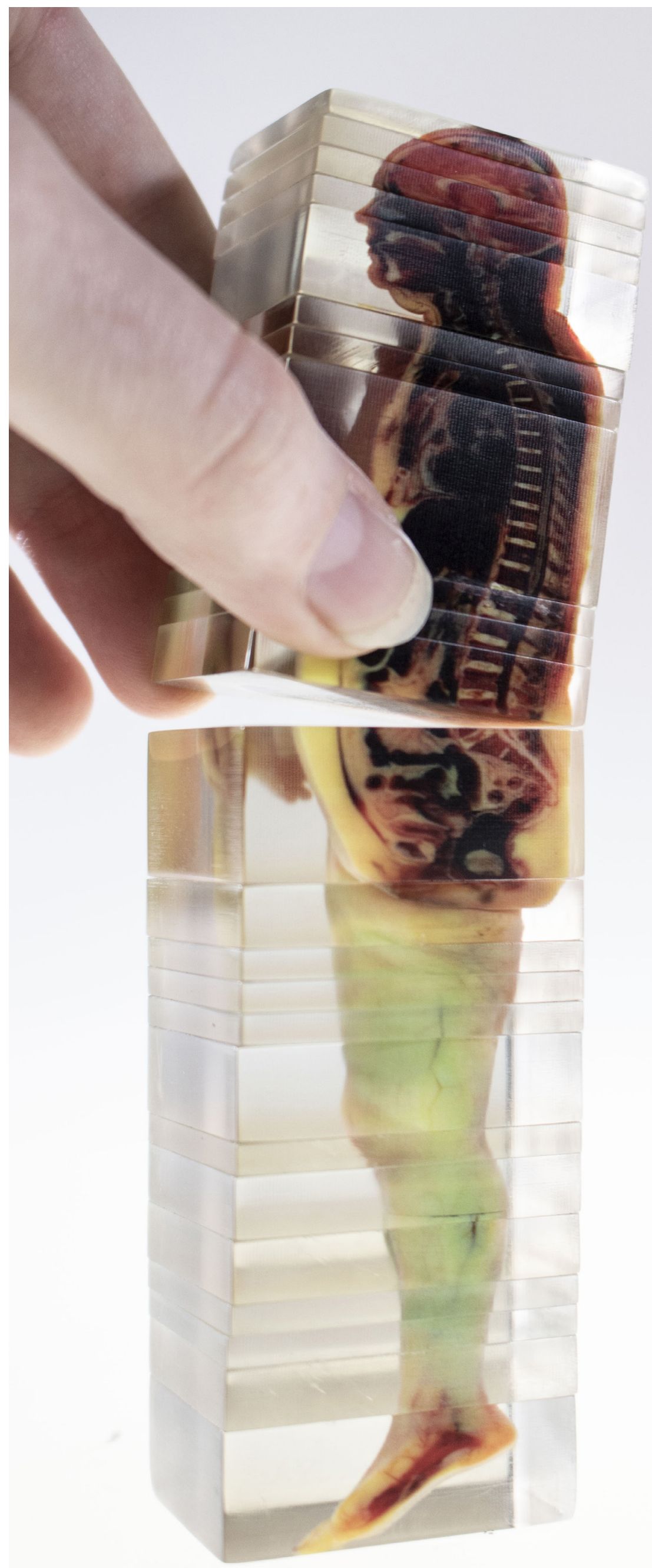




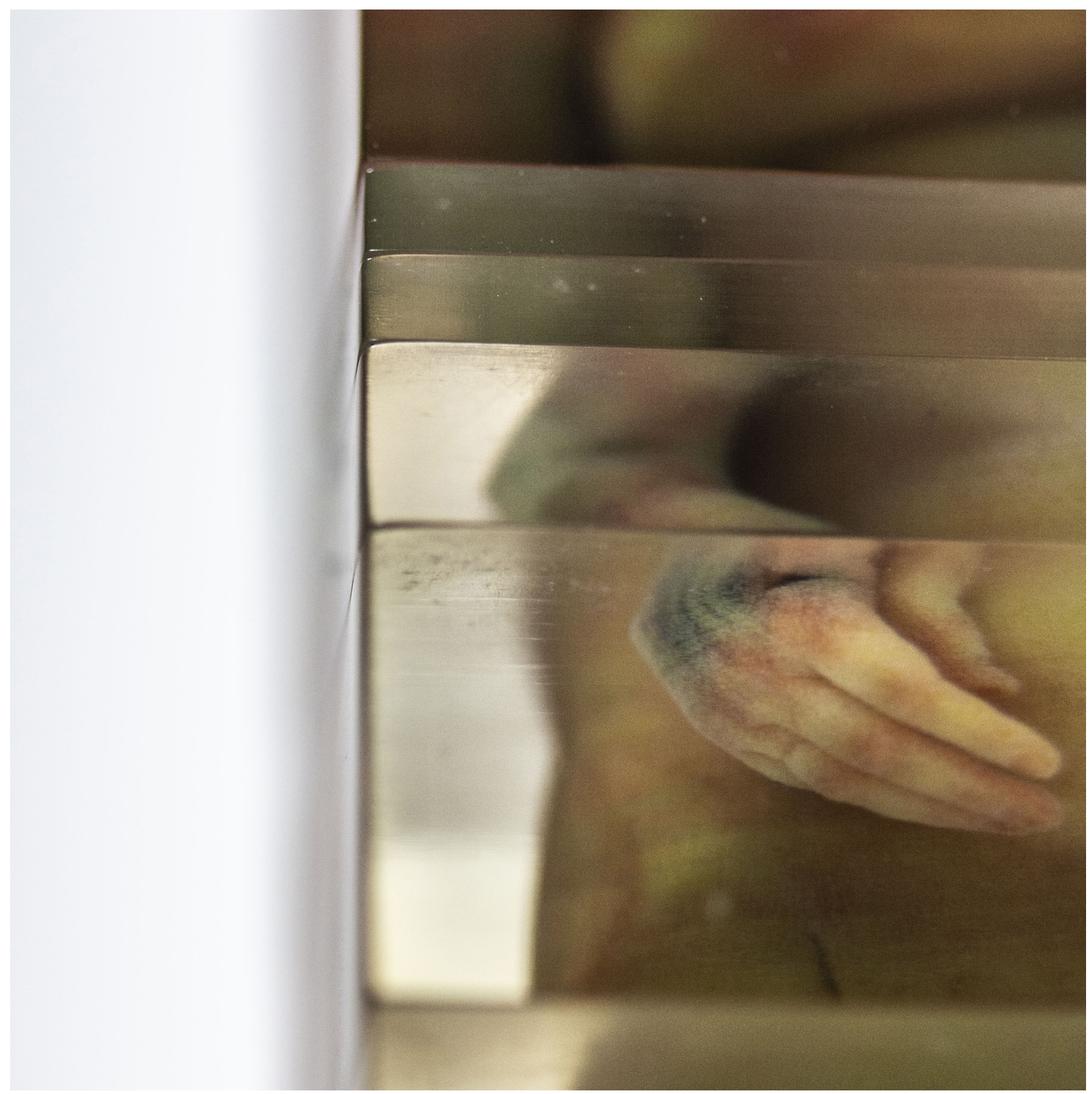



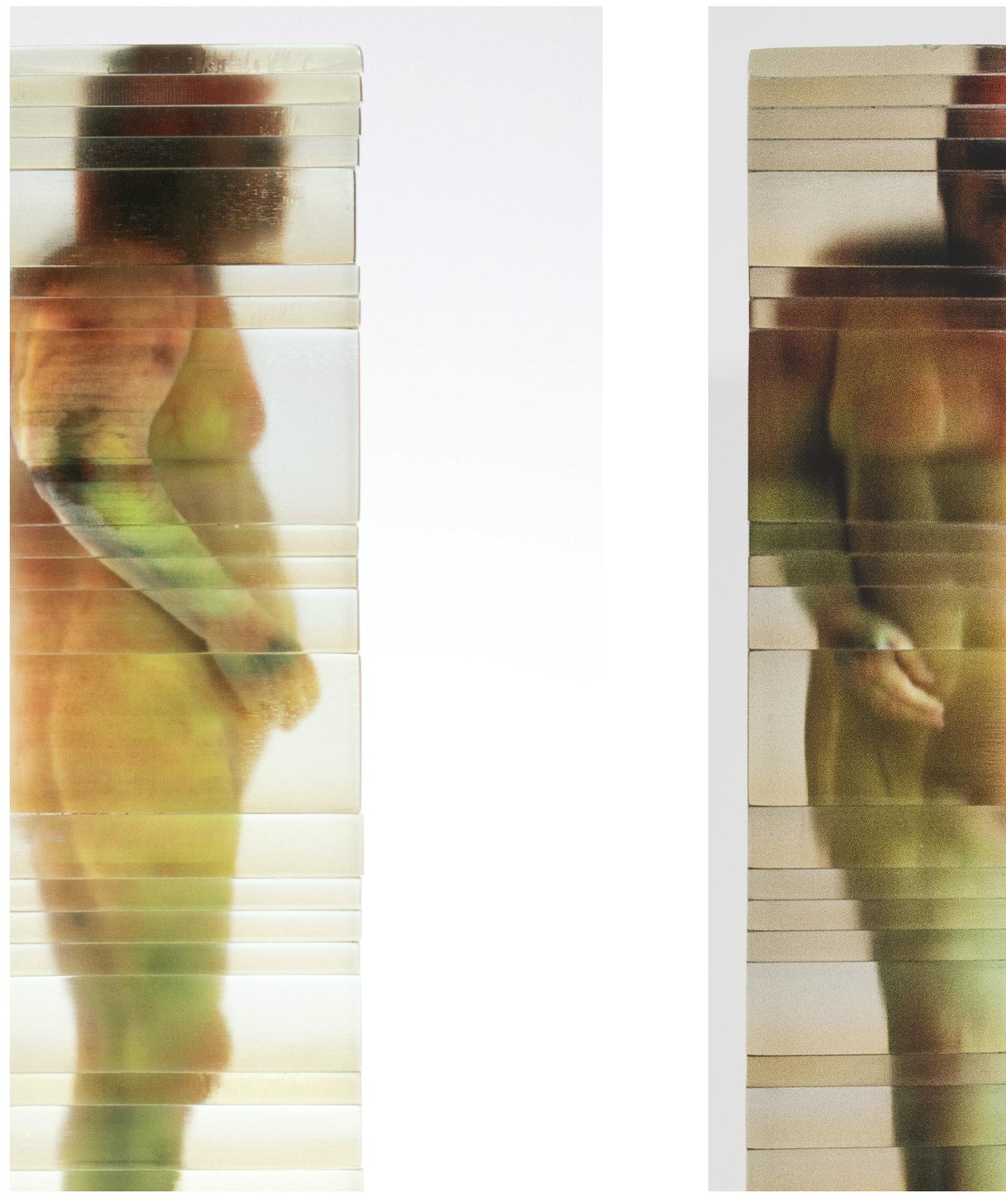

Figure 63. 

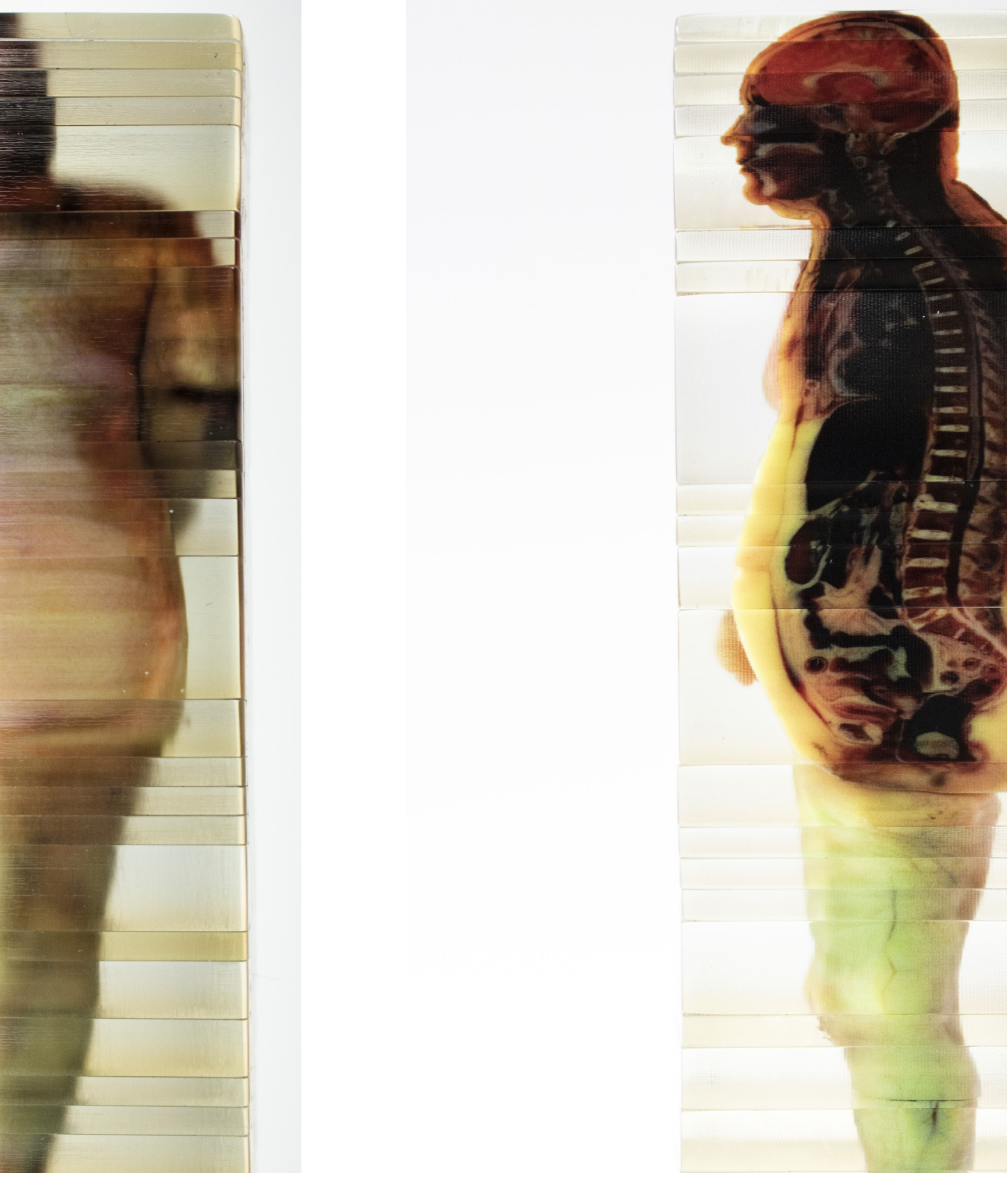

Figure 64.

Figure 65. 


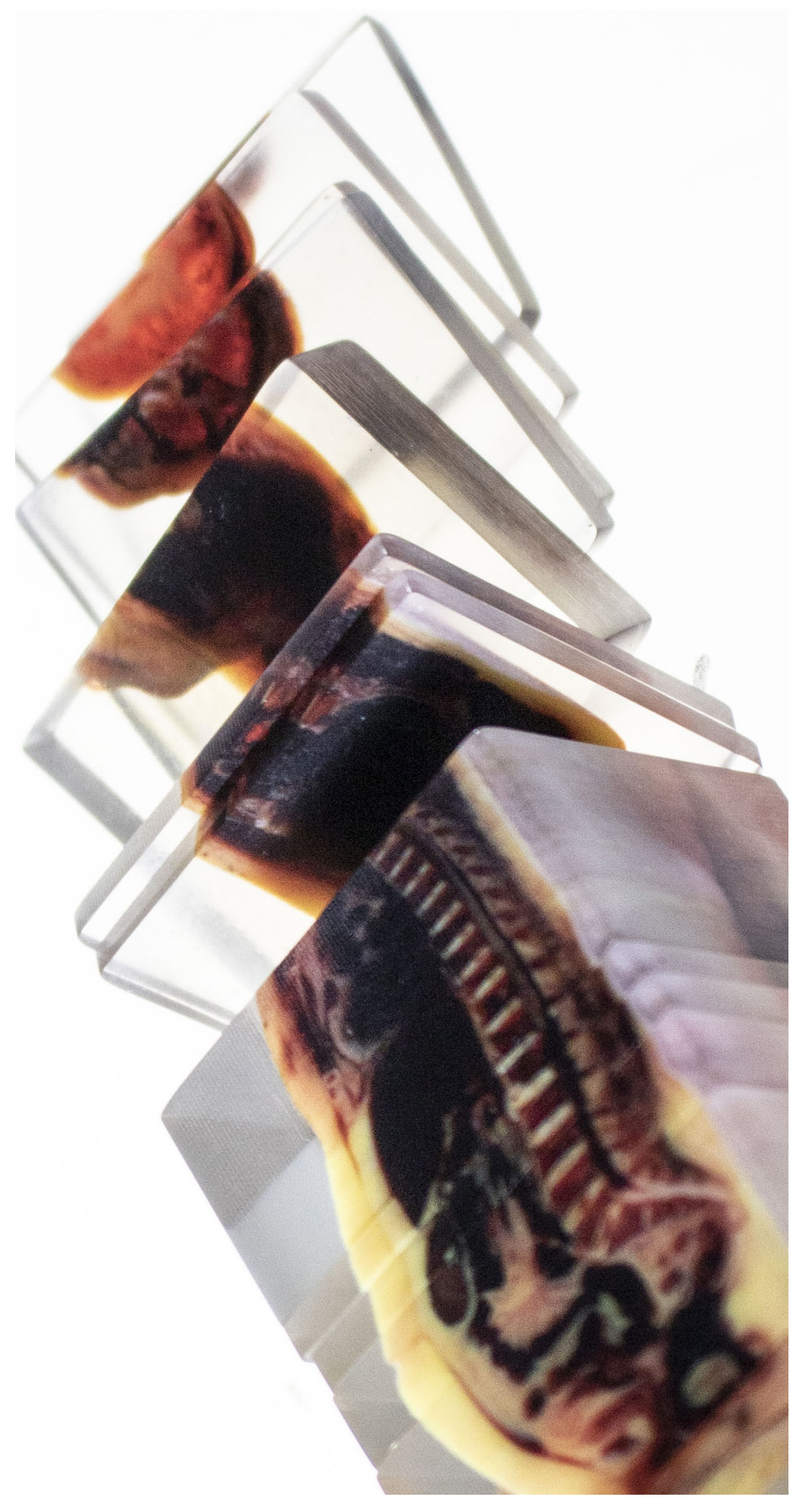




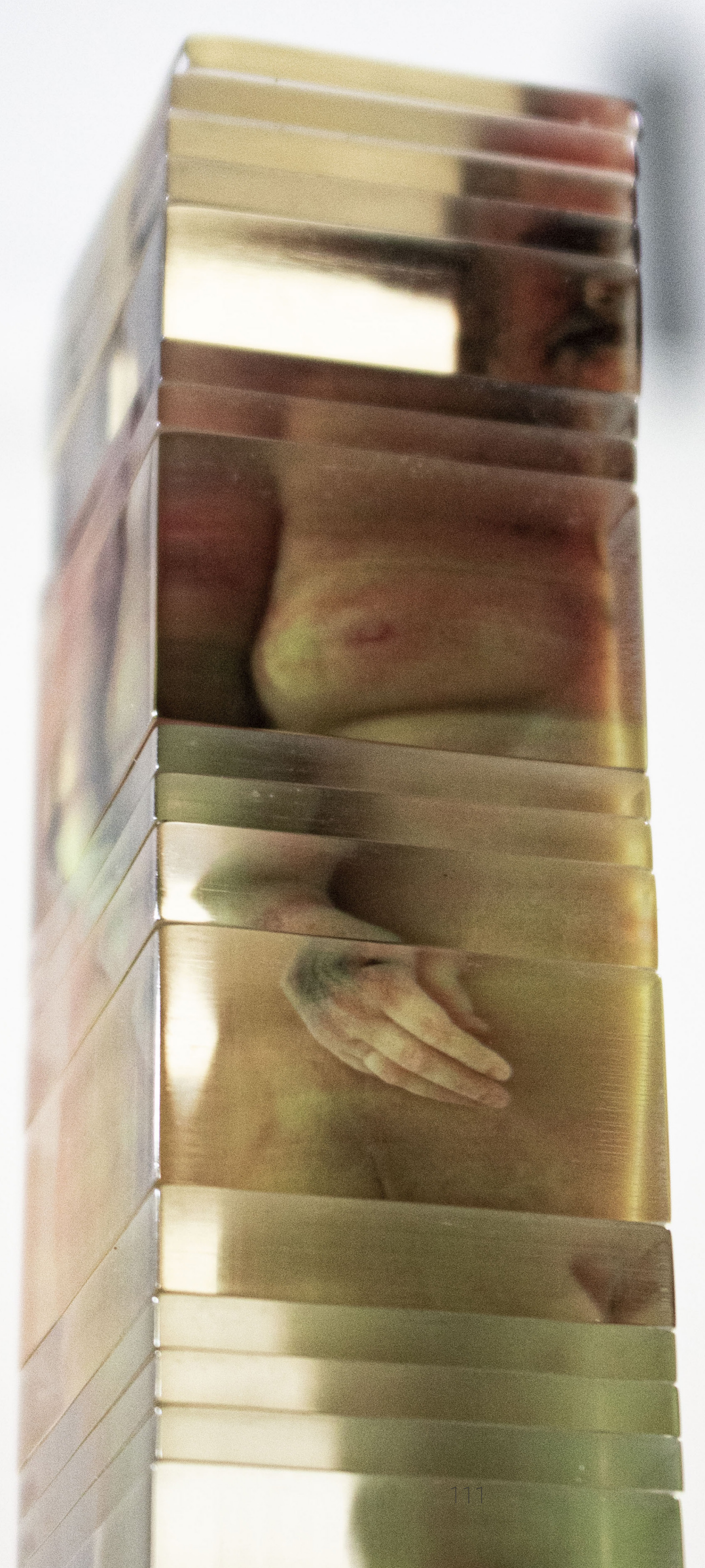

Figure 67. 


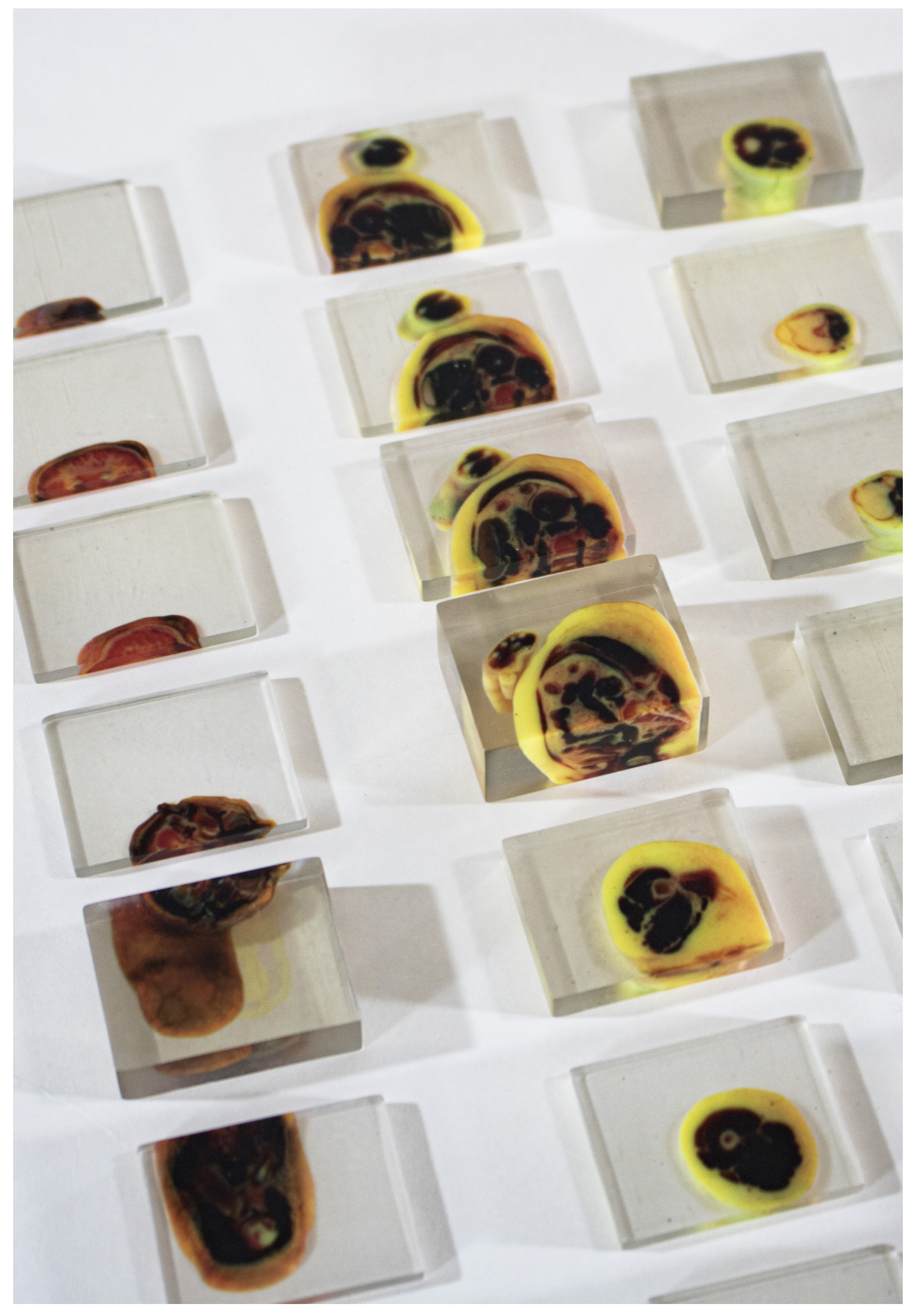




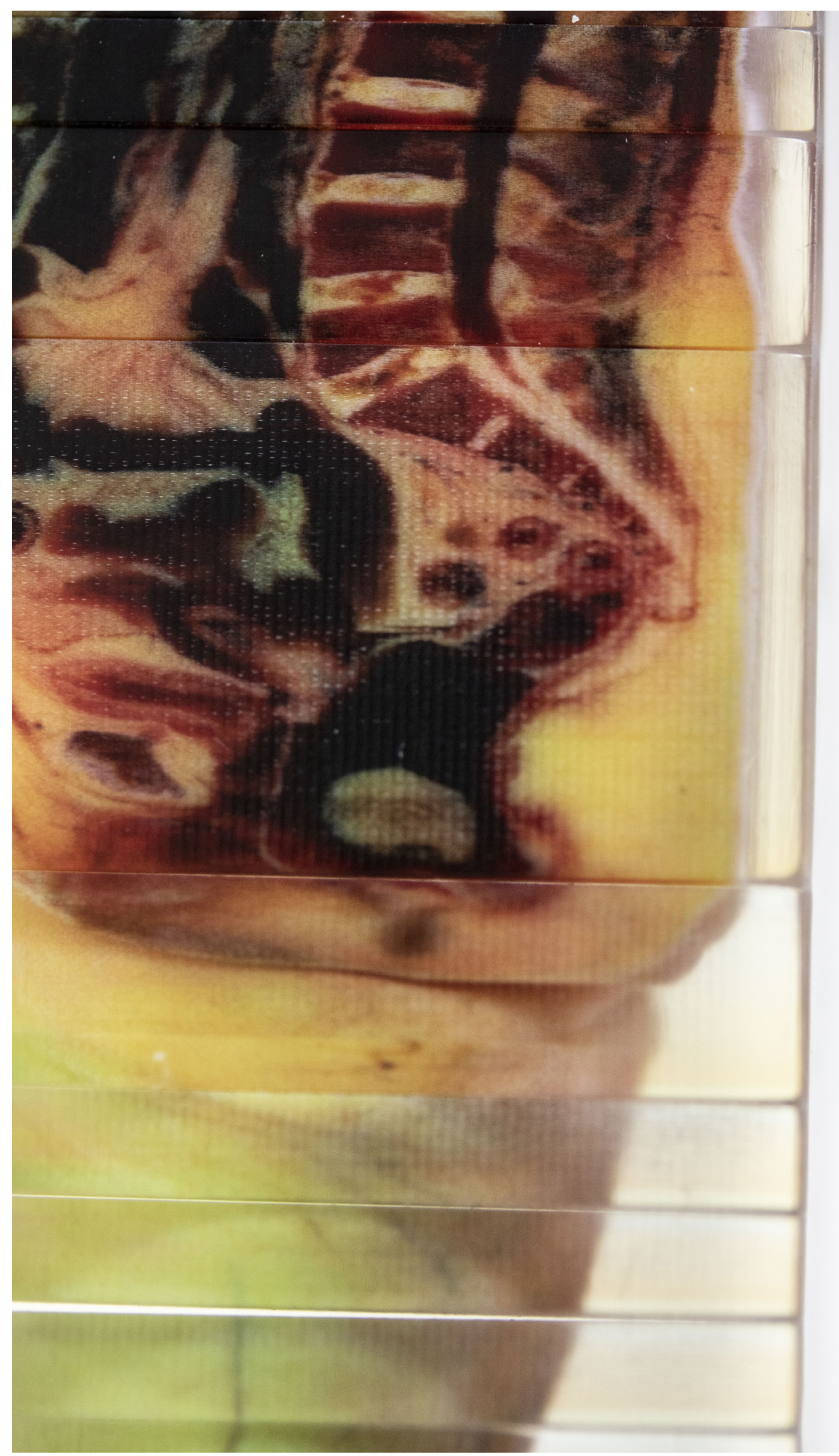




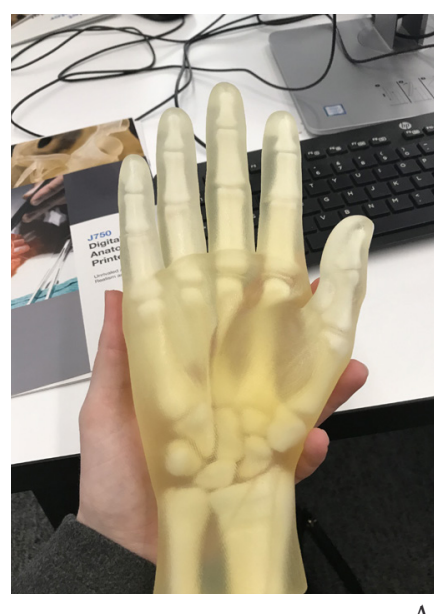

A

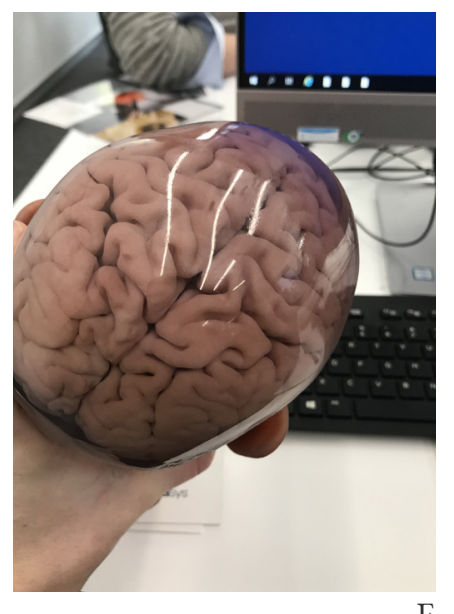

E

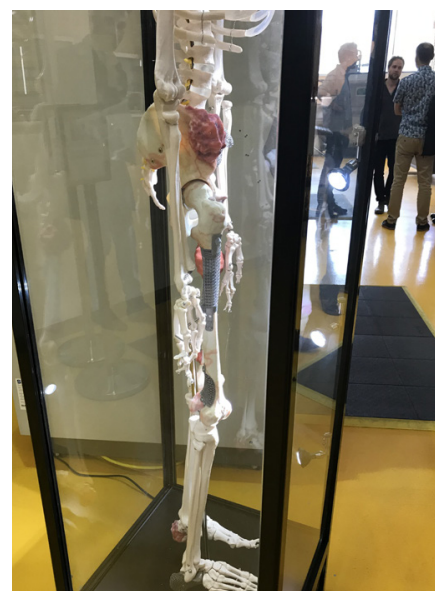

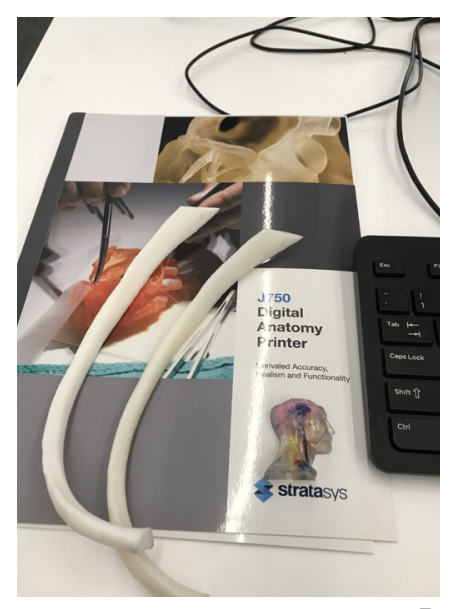

B
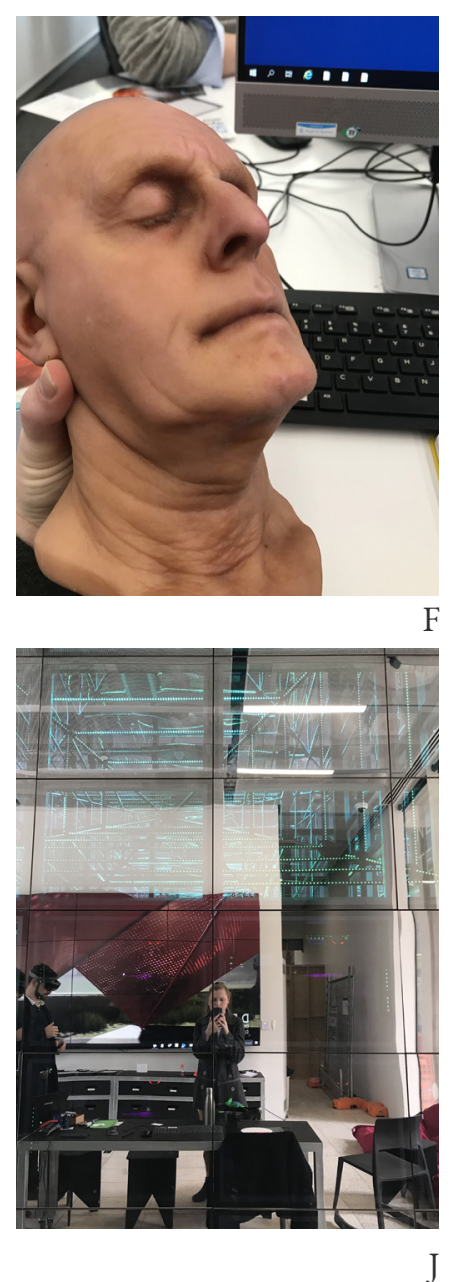

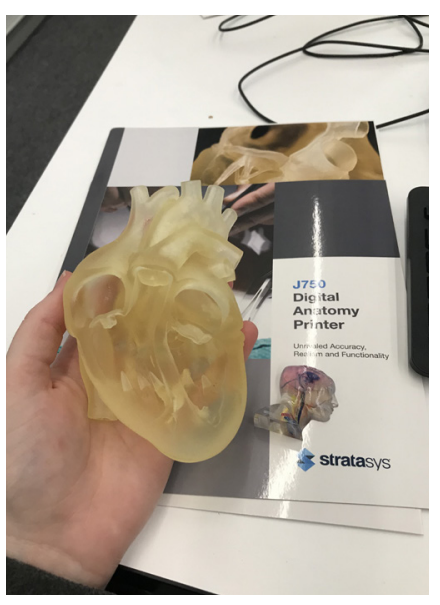

C

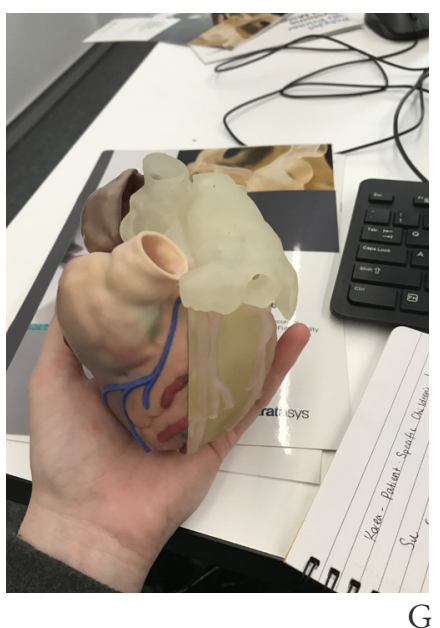

G

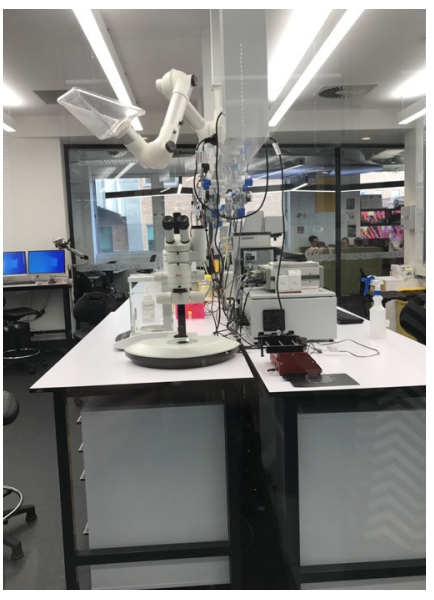

K

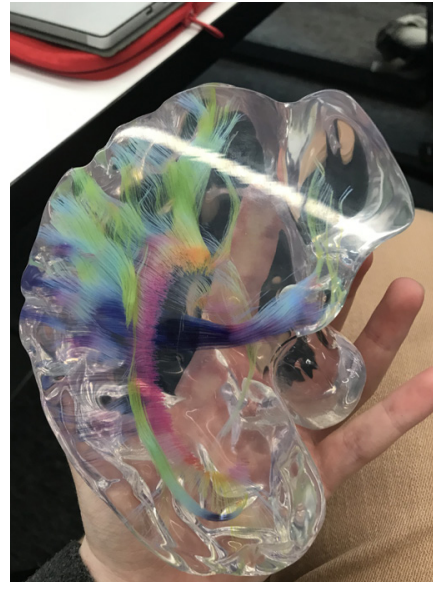

D

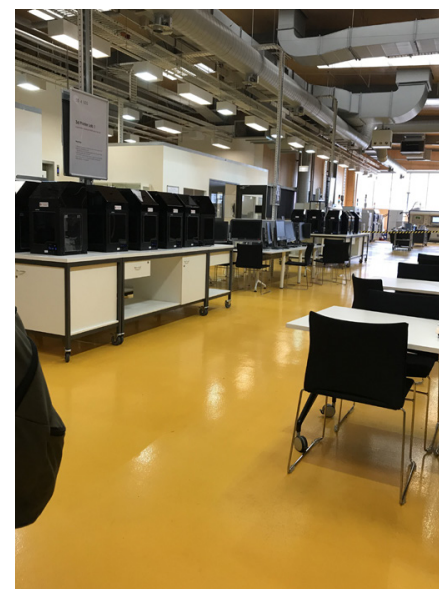

$\mathrm{H}$

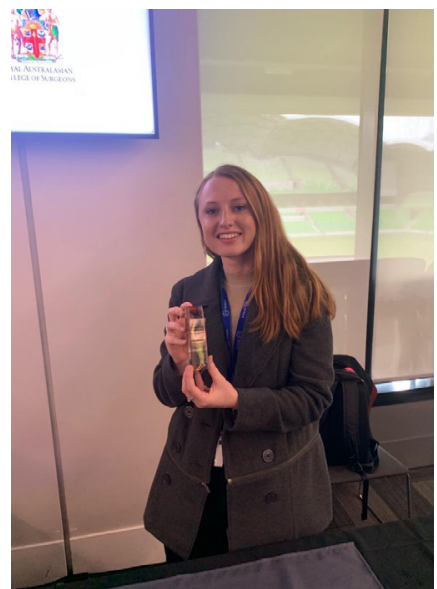

L

Figure 70. Photography taken on the trip to Melbourne for the 3D Technologies in Medicine conference. (A-C) Stratasys J750 ${ }^{\mathrm{TM}}$ Digital Anatomy ${ }^{\mathrm{TM}}$ 3D-printed models. (D-G) Stratasys J750 ${ }^{\mathrm{TM}}$ 3D-printed models shown at the Stratasys presentation. (H-I) Centre for Additive Manufacturing Lab Tour at RMIT including the 3D-printer studio and a 3D-printed skeleton. (J) New Experimental Technology (NExT) Lab Tour at the Melbourne School of Design. (K) Bio-printing lab. (L) A photo of me presenting (taken by the Stratasys marketing team). 


\section{1 .6}

\section{Evaluation and Reflection}

To evaluate this area of research, Case Study 1 was presented at 3D Technologies in Medicine (3DMED $)$ conference in Melbourne, Australia, on 14-16 of November, 2019. Presenters were experts in their respective fields, including highly regarded surgeons, clinicians, designers, VR specialists, medical modellers (including Fusetec), 3D-printing specialists (including Stratasys), biomedical engineers and radiographers from around the world (see Appendix A). The opportunity of presenting Case Study 1, along with attending workshops and receiving feedback from highly esteemed professionals helped to shape the next stage of the research. Visiting design and manufacturing facilities - like the New Experimental Technology (NExT) Lab Tour at the Melbourne School of Design, Centre for Additive Manufacturing Lab Tour at RMIT, SBS Digital Learning Hub, and BioFab3D Tour at St Vincent's Hospital Melbourne - was invaluable to the progress of this research.

Case Study 1 was also presented to specialists ${ }^{2}$ in New Zealand.

MOVING FORWARD

Case Study 1 helped to develop a bitmap-based 3D-printing workflow that can accurately convert serially sectioned images of cryosectioned cadaver into a fullcolour and highly realistic 3D-print of the Visible Female from the VHP.

Full-colour bitmap images were used as 3D-printer inputs instead of traditional mesh-based stereolithographic (STL) file formats to create a 3D-printed model of the Visible Female. The control over every 3D-printed voxel meant that a full colour model of the Visible Female could be created with unprecedented preservation of accurate anatomical colour and detail.

Moving forward, the project will focus on using the developed bitmap-printing workflow to produce models from live patient-specific data with gradated densities. This process is outlined in Case Study 2.

13DMED is Australia's largest 3D technologies in Medicine conference.

${ }^{2}$ including the team at the Auckland Bioengineering Institute (ABI) and the Neurology team at the Wellington Hospital. 


\section{CASE STUDY 1}

REFLECTION AFTER

\section{ATTENDING}

\section{$3 D-M E D$}

\section{THINGS TO CONSIDER}

Case Study 1 helped to develop a bitmap-based 3D-printing workflow that can accurately convert serially sectioned images of cryosectioned cadaver into a full-colour and highly realistic 3D-print of the Visible Female from the Visible Human Project ${ }^{\circledR}$.

Based on the feedback received from the 3DMED conference, it was clear that this method of 3D-printing is relatively new and unprecedented. Most people were unaware of the technique and were interested in its application and potential. The team at Stratasys were impressed by the use of their Voxel-Print Utility.

University anatomy teachers were interested in the application of this model for teaching purposes.

Case Study 1 highlighted potential applications of bitmap-based 3D-printing workflows using biomedical imaging data. This project expands on the VHP's original goal of expanding knowledge into new territory through technologies that offer the potential of generating new biomedical knowledge (The National Library of Medicines Visible Human Project, n.d.).

Anatomically accurate colour was achieved because of the discovery that the VeroClear ${ }^{\mathrm{TM}}$ can severely change the colour of the 3D-print, making the print darker and green.

I talked to the team at Materialise Mimics, Stratasys, 3Dsystems, and Auckland Hospital's 3D-Print Lab.
Many suggestions were given for the future direction of this project. Case Study 1 drew interest from surgeons, clinicians and biomedical engineers because this workflow could potentially incorporate patient-specific biomedical data (such as CT and MRI datasets) as well as integration of both soft (Agilus30 $30^{\mathrm{TM}}$ ) and rigid (Vero ${ }^{\mathrm{TM}}$ ) materials in bitmap-based file formats. There was interest in creating gradients in material densities as many 3D-printed medical models are used for device testing, surgical simulation and skills acquisition and biomechanical research purposes and therefore realistic anatomical movement would be useful.

Some experts implied that replicating anatomical density through bitmap-based 3D-printing could be an extremely useful tool, especially if the models could be created by using live patient-specific data.

Stratasys released the Stratasys J750 ${ }^{\mathrm{TM}}$ Digital Anatomy ${ }^{\mathrm{TM}}$ 3D-Printer at the 3DMED conference with new anatomical materials. Combining a bitmap-based 3D-printing workflow with the new Stratasys anatomical materials could produce anatomically accurate $3 \mathrm{D}$-prints at a 14 -micron resolution. 


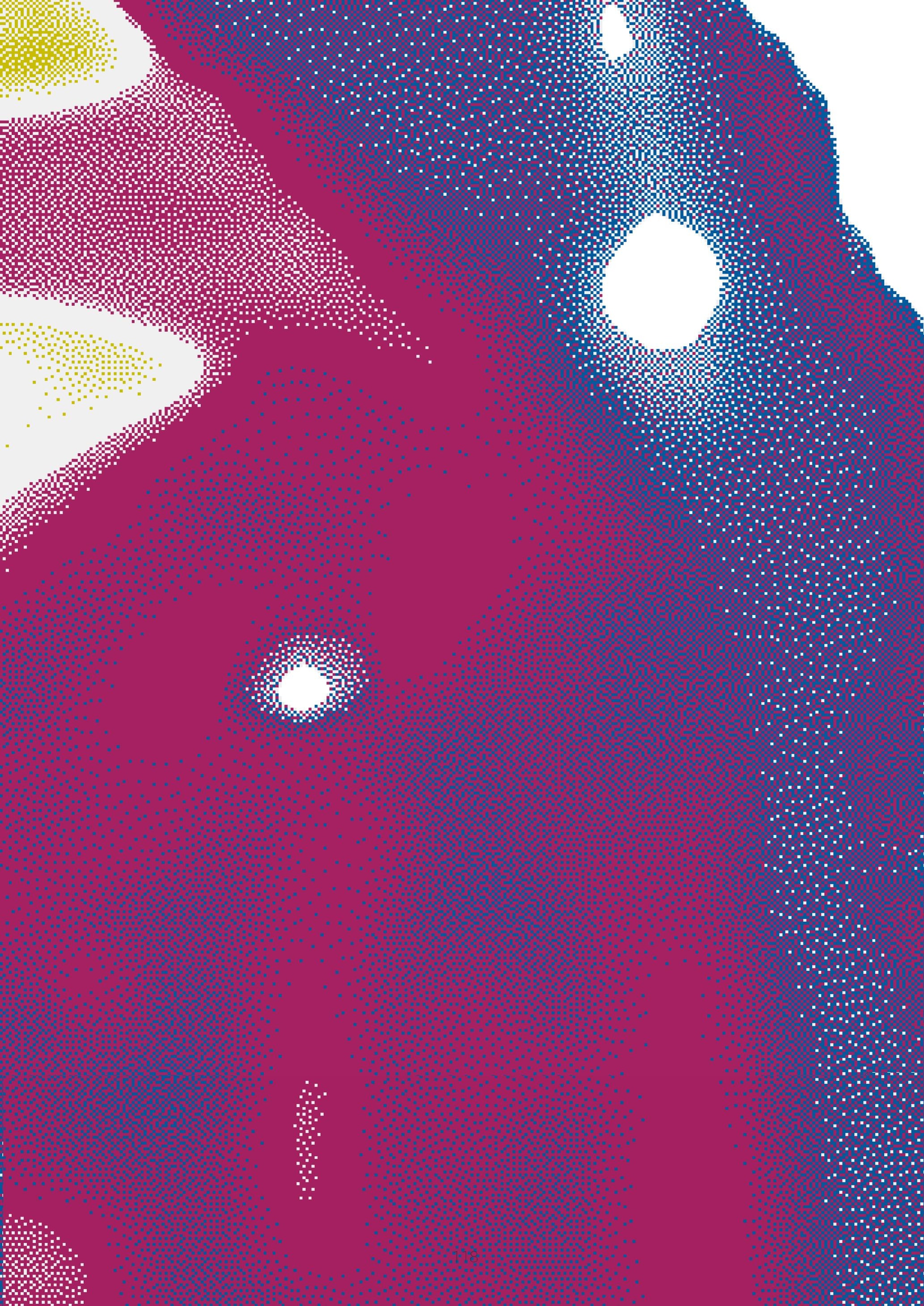


PRACTICAL ACTION RESEARCH

\subsection{A S E S T U D 2}

Replicating the Dynamic Movement of Human Anatomy using CT Imaging Data and a Bitmap-based 3D-Printing Workflow.

Left:

Figure 72. Digitally halftoned image of bitmap-based 3D-printed neonate. 


\section{overview}

Case Study 2 focused on replicating the biomechanical movements of human anatomy by using patient-specific CT data and a streamlined bitmap-based 3Dprinting workflow. The research focus shifted from replicating anatomical colour to mimicking anatomical density and biomechanics in 3D-printed models based on feedback and reflection regarding Case Study 1. This meant that the bitmapbased 3D-printing workflow developed in Case Study 1 was altered to suit the new set of criteria developed for Case Study 2.

Models of neonatal anatomy have potential value, especially in the areas of surgical models for trainee education, open surgery and planning (Stramiello et al., 2020). Adequate training can help mitigate risks associated with complex paediatric intervention, improving survival and life quality, especially in perinatal acute care (Meurs et al., 2003). Due to the gap in knowledge around neonatal modelling, biomechanics and the limited amount on neonatal simulators available on the market, Case Study 2 focussed on mimicking realistic movement in neonatal anatomy. Currently, paediatric and neonatal simulators "are of low physical fidelity therefore cannot provide qualitative insight into the procedure" (Thielen et al., 2019, p. 1).

Paediatric anatomy is intricate and data loss during the segmentation process can be detrimental to the quality of the 3D-print. Bitmap-based 3D-printing showed promise for neonatal modelling and paediatric high-fidelity dynamic modelling.

Anatomical density is captured through CT imaging rather than serially sectioned cadaver (such as the VHP). Turning greyscale images into volumetric models for 3D-printing using a bitmap-based workflow presents various challenges, such as the accurate replication of anatomical density and movement. However, one of the main advantages of using CT imaging data is that dynamic and tangible 3D-models can be produced from living patient data rather than from cadaveric material.

Cross-sectional CT images of anatomy can generate volumetric or a "voxel representations" of body parts by using an interpolation function that fills in missing pieces between CT layers. Interpolating, re-slicing and exporting CT image files at the Stratasys $\mathrm{J} 750^{\mathrm{TM}}$ 3D-Printer native $\mathrm{z}$ resolution meant that 3D-printed models of anatomy were successfully produced from CT imaging data using a bitmapbased 3D-printing workflow. 


\section{2 .2}

\section{Criteriafor casestudy 2}

The overall focus of Case Study 2 was to outline the most successful workflow for converting CT imaging data into dynamic bitmap-based 3D-printed models that mimic human anatomical movement.

C RITERIA

\section{Select an area of neonatal anatomy that will serve as a starting point} for design exploration.

The chosen area of anatomy should contain a range of gradients (soft and hard areas) to test the material properties of the Stratasys J750 ${ }^{\mathrm{TM}} 3 \mathrm{D}$-Printer and a bitmap-based 3D-printing workflow

2. Explore and develop a bitmap-based 3D-printing workflow using a patient-specific biomedical imaging dataset.

A bitmap-based 3D-printing workflow should be developed to 3D-print sections of neonatal anatomy with anatomically accurate density. The workflow should be streamlined and work to create extremely detailed prints with integrated dynamics using a limited amount of processing steps and programmes.

Material ratios should be noted and addressed throughout the design process to develop a high-fidelity final bitmap-based 3D-print of the selected area of anatomy.

\section{Use the most effective bitmap-based 3D-printing workflow to create a high-fidelity 1:1 dynamic medical model of an area of anatomy using a patient-specific biomedical imaging dataset. \\ By blending Vero ${ }^{\mathrm{TM}}$ (hard) and Agilus30 ${ }^{\mathrm{TM}}$ (soft) using a bitmap-based 3D- printing workflow, the level of realism in the Final Design should be unprec- edented to provoke thought about the future of 3D-printing in medicine. The final 3D-print should be evaluated by a process of self-reflection and discus- sions with expert opinions.}


The dataset used in Case Study 2 was a high-resolution C'T scan of a neonate. The area of anatomy chosen for initial experimentation was the foot. This is because a neonatal foot includes varying densities, from soft fat to hard bone. Biomechanical movement of the foot can be early indicators of conditions such as cerebral palsy and equinovarus (clubfoot) which develop in neonates (Aurell et al., 2002; Heineman et al., 2011). Therefore, the foot was a logical area for design exploration, especially for a designer without formal medical training.

Testing different bitmap-based 3D-printing workflows for the purposes of mimicking anatomical density from CT imaging data occurred through Parallel Prototyping. Once an appropriate workflow was established, further Parallel Prototyping and Focussed Prototyping occurred.
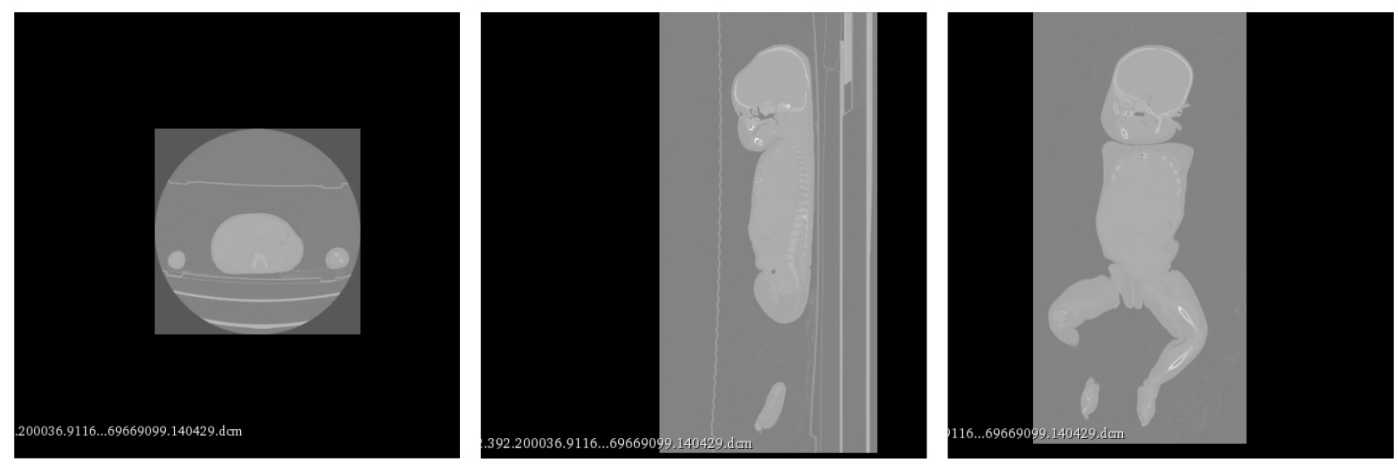

Figure 73. Bitmap baby CT scan (three planes).

DIGITAL WORKFLOW

The dataset was downloaded and volumetrically reconstructed to create a virtual model and an interpolation function was used to fill in missing slices to match the native $3 \mathrm{D}$-printer $z$ resolution. The prototyping process followed the workflow outlined in Figure 74. A total of 483 images were processed to complete each foot swatch. 
FOLDER 1

(Columetric data in a folder (CT dataset).

STAGE ONE: Volumetrically

Reconstructing Medical Data

a. Enter volume reconstruction

b. Use the Gradient Anisotropic

Diffusion Module to smooth surfaces.

c. Centre Volume.

d. Rotate volume and Camera 1 to correct orientation

e. Volumetrically reconstruct model using a Volume Rendering Module (Interpolation)

f. Assign materials and determine threshold.

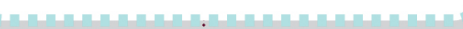

g. Crop specific area of anatomy.

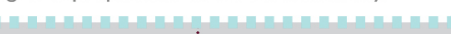

h. Export Images as PNG (bitmap) image files at the native printer $z$ resolution ( 14 micron or 27 micron).

\section{FOLDER 2}

i. Save the images in a folder on the computer.
STAGE TWO: Batch Processing (automated workflow)

a. Enter batch processing software.

b. Open the actions tab and create a new action.

Name Action

- Open an image from FOLDER 2 (select the image with the largest surface area).

- Crop image to include the boundary box (this can be a general boundary box or the subject).

Make sure there is no background.

- If applicable, you can scale the image in this step.

- Image $\longrightarrow$ Image size $\longrightarrow$ Change the image size to $3 \mathrm{D}$-printer $\mathrm{XY}$ resolution.

- If applicable, you can scale the image in this step. Make sure that the size of the anatomy $(\mathrm{mm})$ is accurate. If not, this needs to be adjusted.

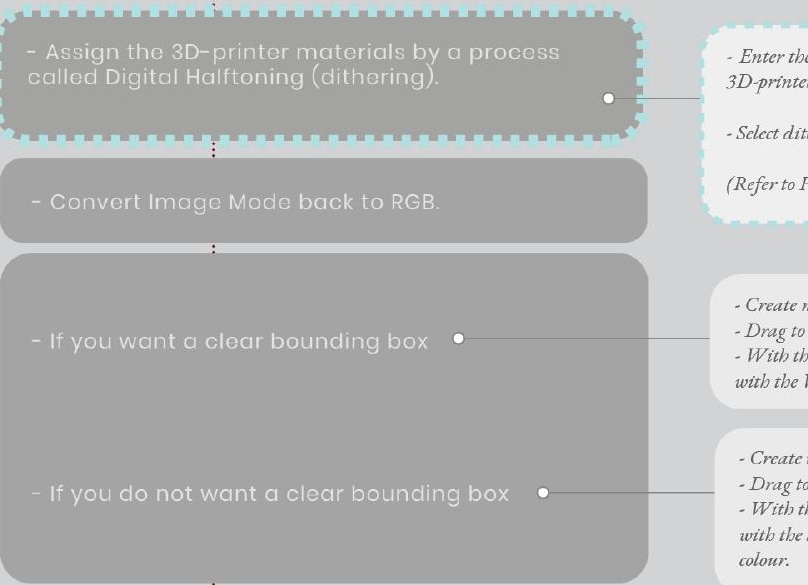

FOLDER 3

FOLVE the inage as a DNG file (Save as $\rightarrow$ )

Create a new folder for the digitally halftoned images and save in this folder).

- Close

Select Don't Save.

c. Automate the Batch Process to Digitally Halftone all images.

File $\longrightarrow$ Automate $\longrightarrow$ Batch Process.

- Select Action. 


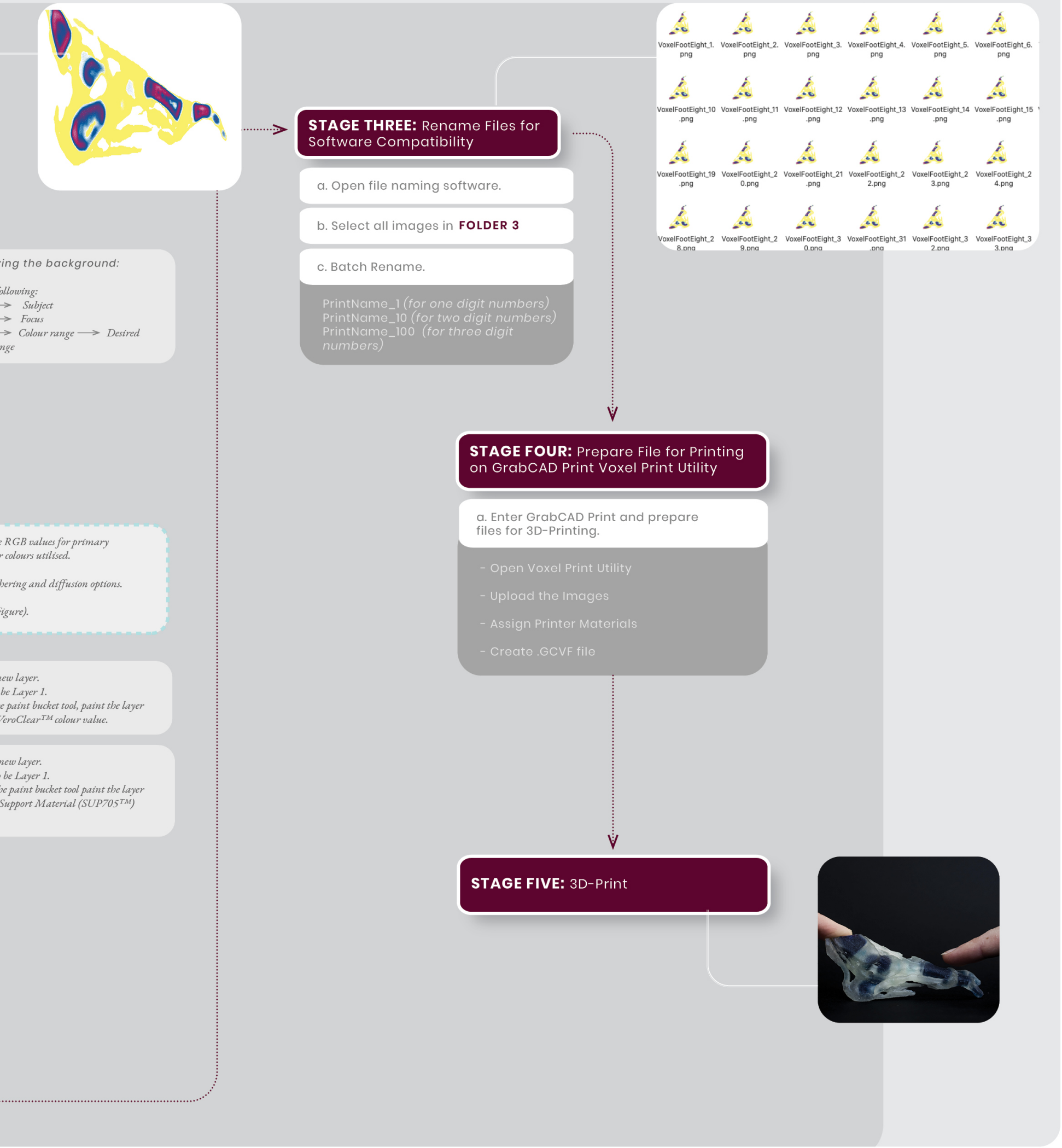




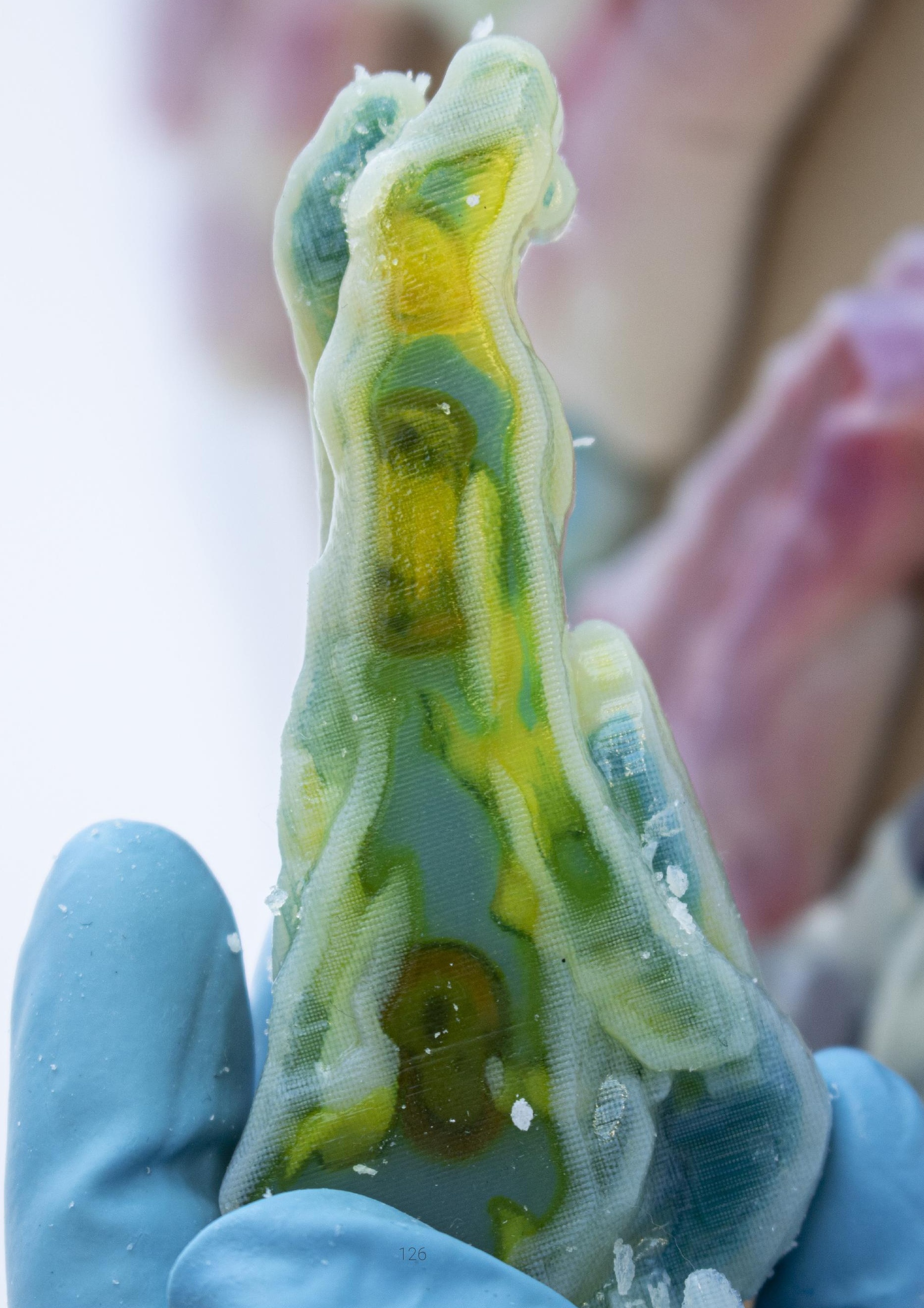


4.2. 4

Design Experimentation

Note: Vero ${ }^{\mathrm{TM}}$ material colours used throughout this process are interchangeable. Colour was used to identify each print from one another since they are all the same geometry and can easily get mixed up.

Left:

Figure 75. Neonatal foot swatch covered in support material before cleaning. 
Volumetric Reconstruction (Stage One of Digital Workflow)
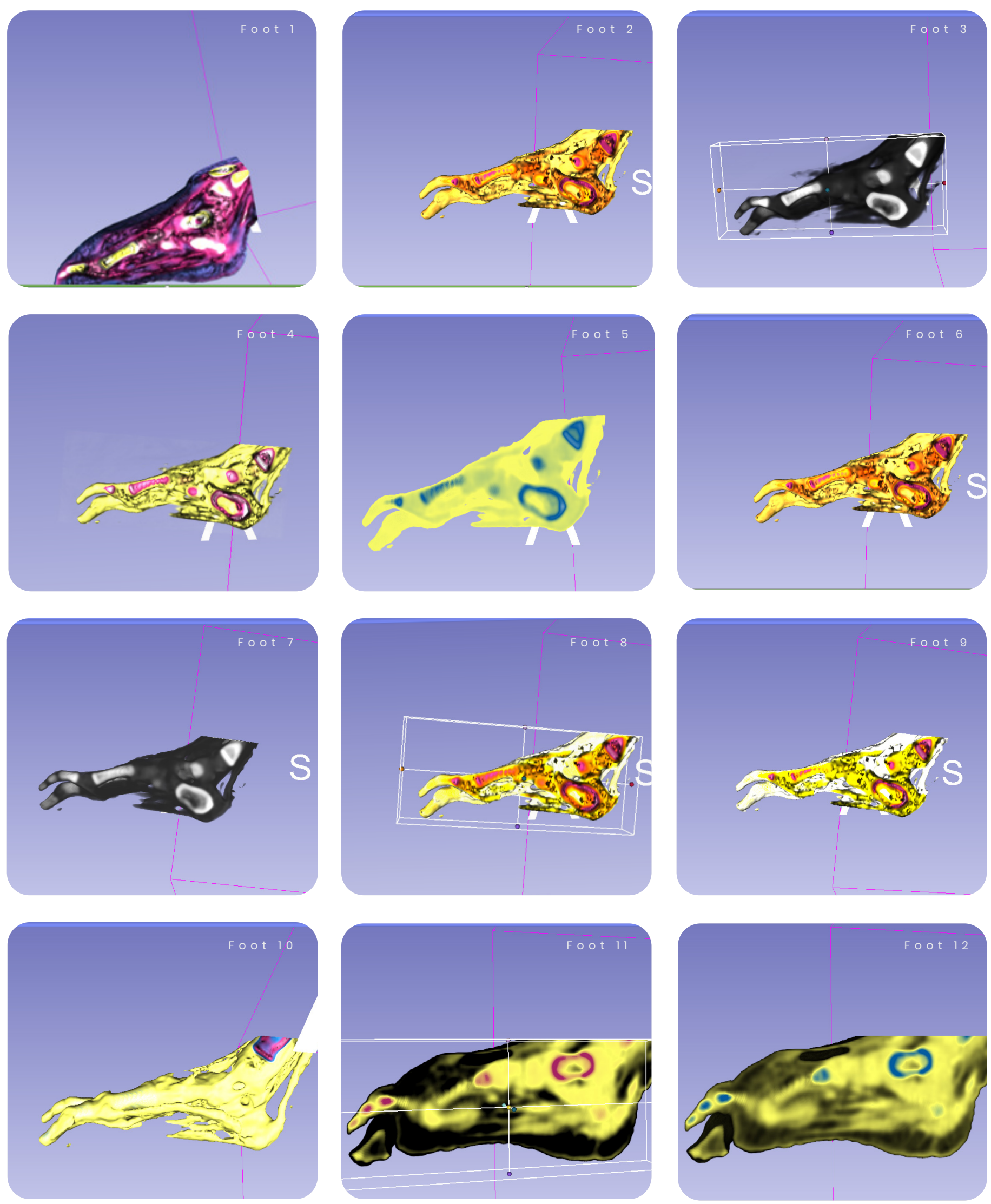

Figure 76. Volumetric reconstructions of neonatal foot (cross-section) with integrated 3D-print settings. 
Digital Halftoning (Variable step in Stage Two, part b of Digital Workflow)
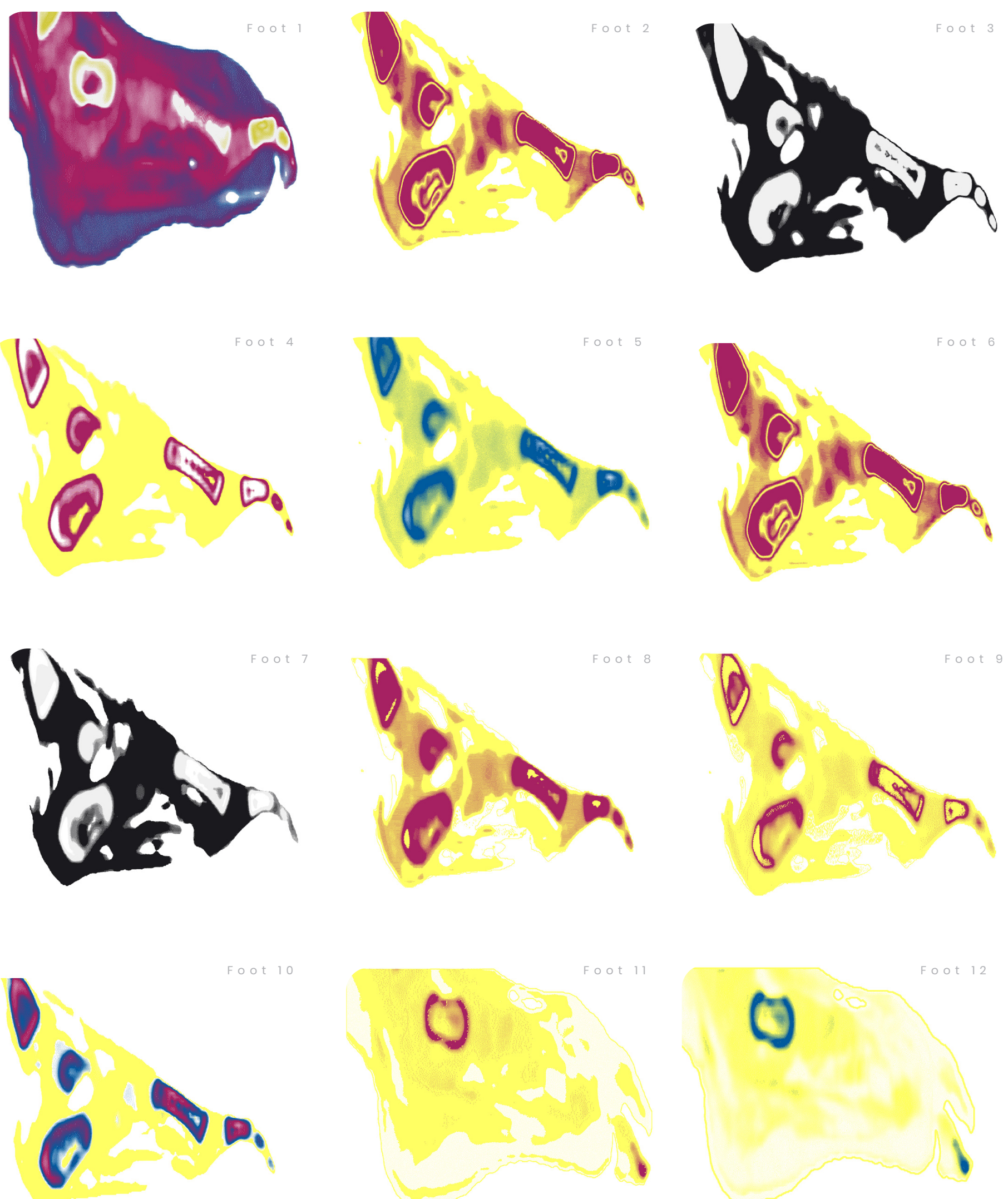

Figure 77. Cross-sectional 14-micron image slices of digitally halftoned neonatal feet. 


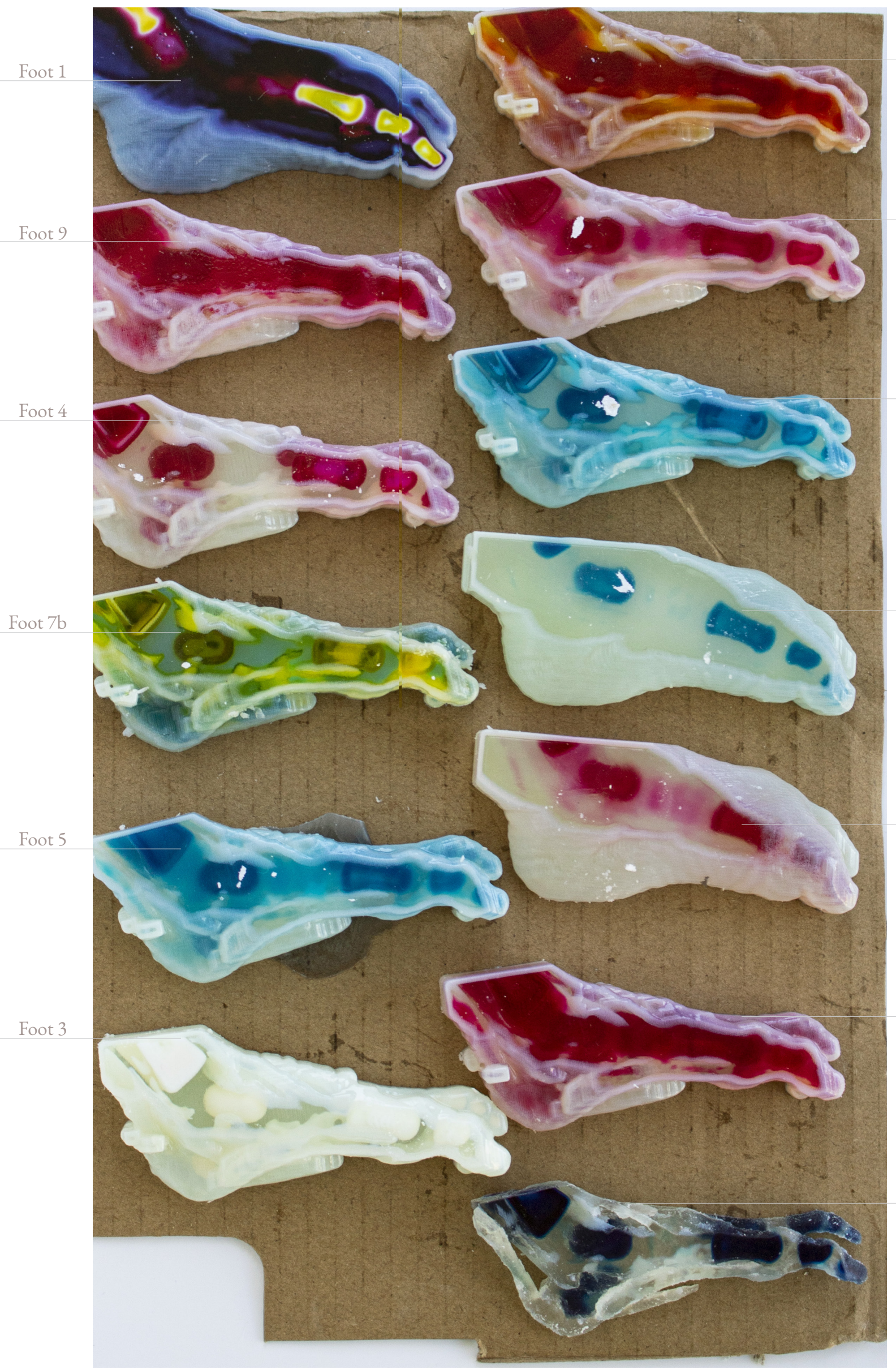

Foot 2

Foot 8

Foot $7 \mathrm{a}$

Foot 11

Foot 12

Foot 6

Foot 10

Figure 78. Cross-sections of bitmap-based 3D-printed neonatal feet prototype models (two toes) straight off the 3D-pritner bed and coated in SUP706 ${ }^{\mathrm{TM}}$. 


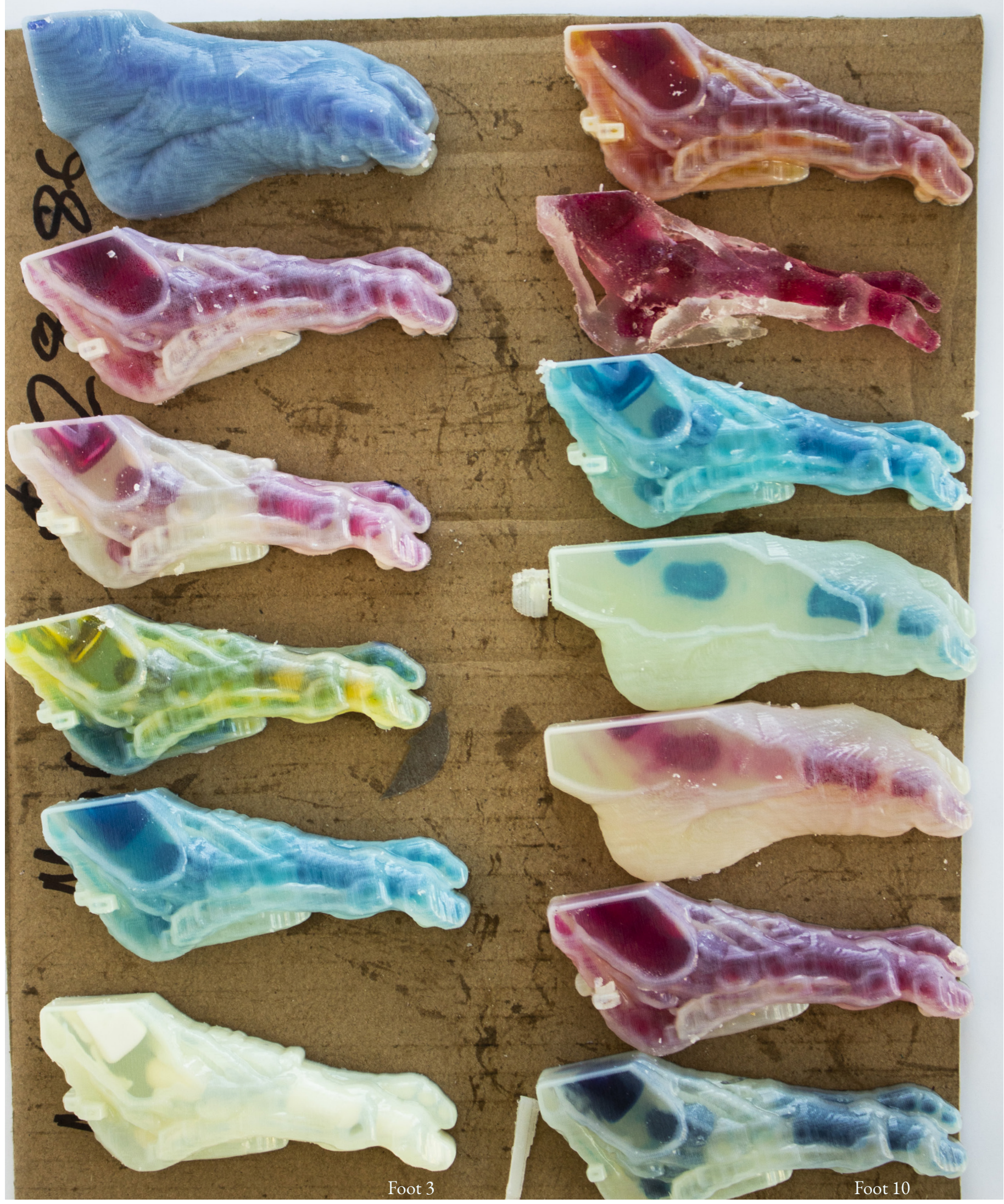

Figure 79. Bitmap-based 3D-printed neonatal feet prototype models (two toes) straight off the 3D-pritner bed and coated in SUP706 ${ }^{\mathrm{TM}}$. 

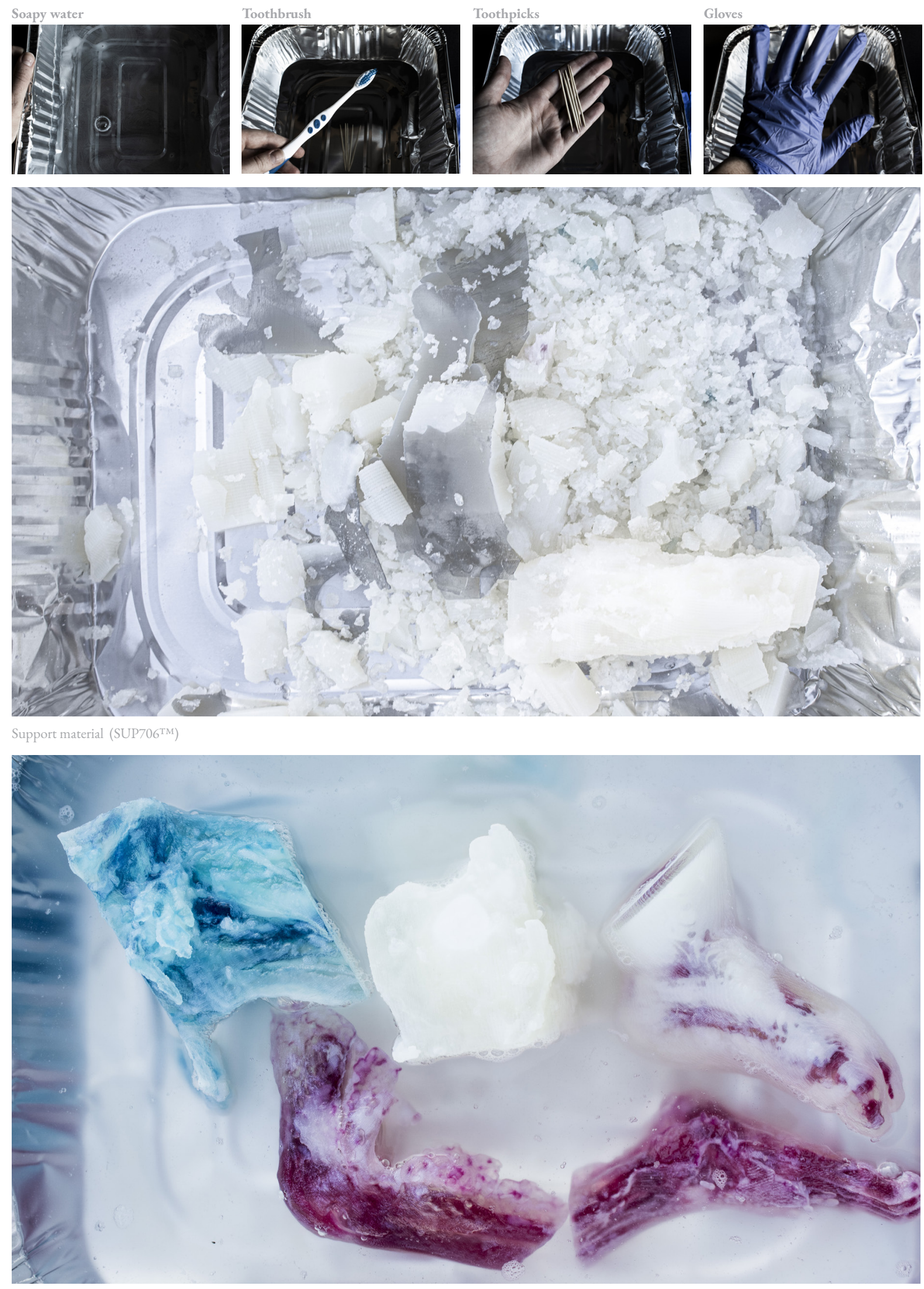

Figure 80. Cleaning 3D-prints. 
The SUP706 ${ }^{\mathrm{TM}}$ cleaning process can be challenging due to some intricacies in 3D-print structure, especially in anatomically complex models. The post-processing workflow used in Case Study 2 is outlined below.

1) The 3D-prints were soaked in warm water with a small amount of dishwashing liquid to gently remove large clumps of SUP70 $6^{\mathrm{TM}}$.

2) Once the large pieces of support material were removed, using a toothpick and toothbrush, the 3D-prints were brushed softly and any SUP706 ${ }^{\mathrm{TM}}$ was scraped away on a dry surface to remove SUP706 ${ }^{\mathrm{TM}}$ film left on the 3D-print. The object was dipped into a container full of warm water and dishwashing liquid periodically to remove any loose SUP706 ${ }^{\mathrm{TM}}$ fragments.

3) The 3D-prints were then placed in a chemical cleaning bath available at the School of Design Innovation. The chemical cleaning bath dissolves support material in delicate areas of the print that cannot be removed by hand. If the objects are left in the chemical cleaning bath for too long, they can warp in shape, so it is important to consider this (approximately 1 hour is a good amount of time). 

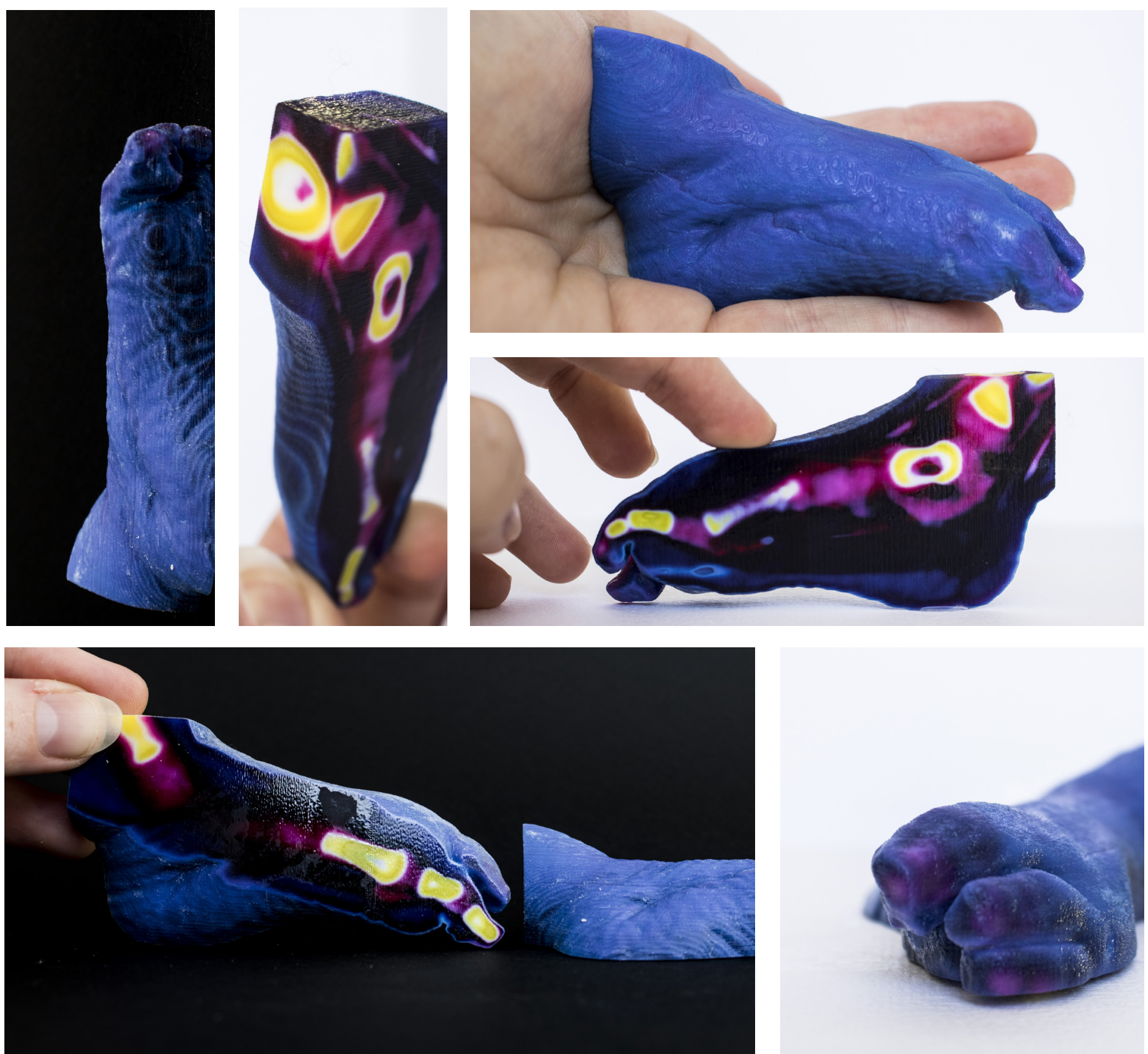

\section{A i m}

To 3D-print with purely Vero ${ }^{\mathrm{TM}}$ materials (similar technique to Case Study 1) to understand which materials blend using bitmap-based 3D-printing from CT datasets (due to its translucency Agilus30 ${ }^{\mathrm{TM}}$ makes it hard to see if materials are blending). The full neonatal foot was processed.
Discovery

Materials blended well with a high level of detail. The addition of VeroPureWhite ${ }^{\mathrm{TM}}$ made the 3D-print opaque and therefore some areas are not translucent, and details are hidden because of this. 

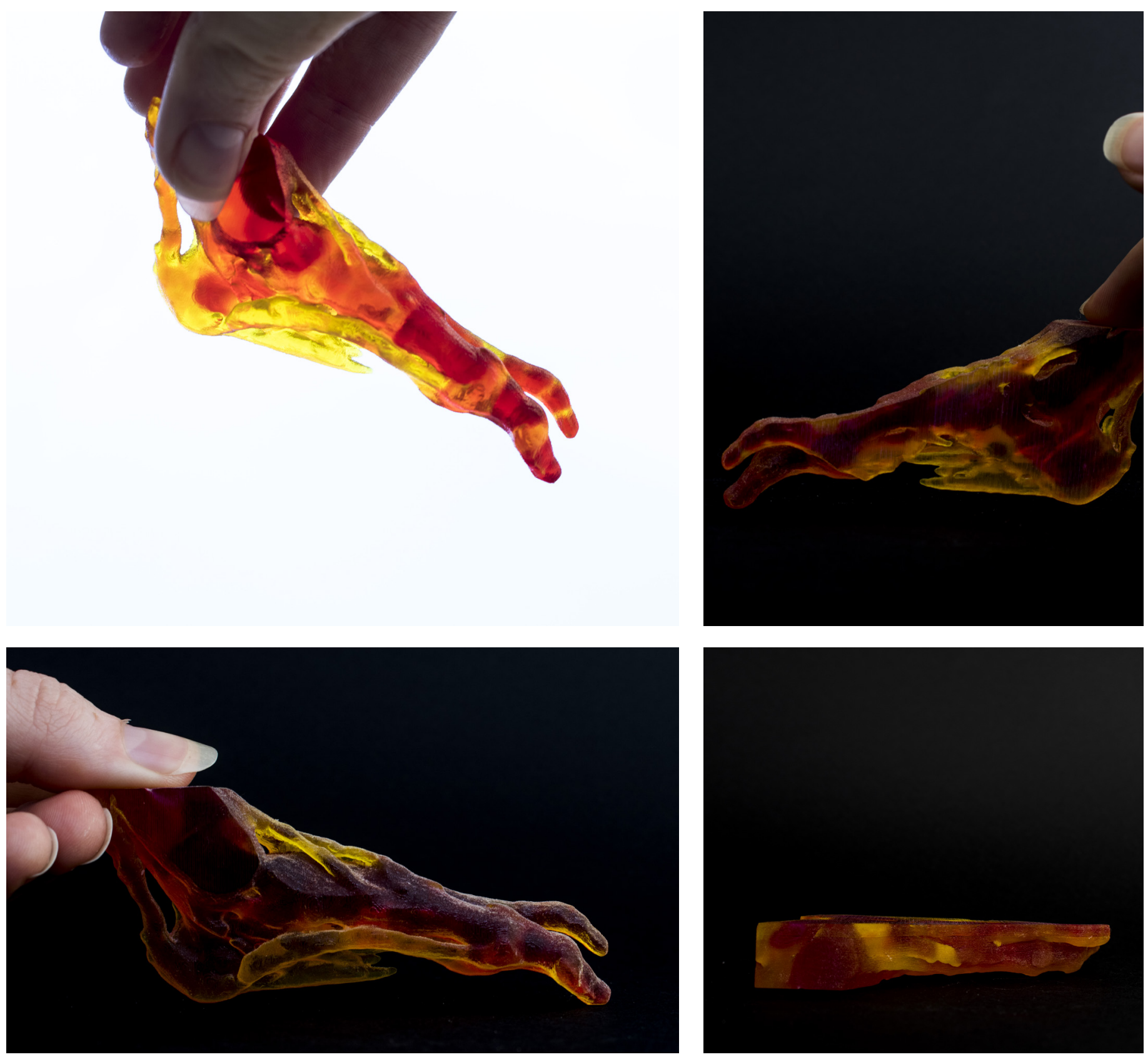

Figure 82. 3D-print\#15.

A i $m$

Same aim as 3D-print \#14 but only the soft tissues were processed (not including fat and skin).
Discovery

Removing the addition of VeroPureWhite $^{\mathrm{TM}}$ meant that the 3D-print was translucent and the blend between the two materials (VeroMagenta-V $V^{\mathrm{TM}}$ and VeroYellow- $\mathrm{V}^{\mathrm{TM}}$ ) is evident. 

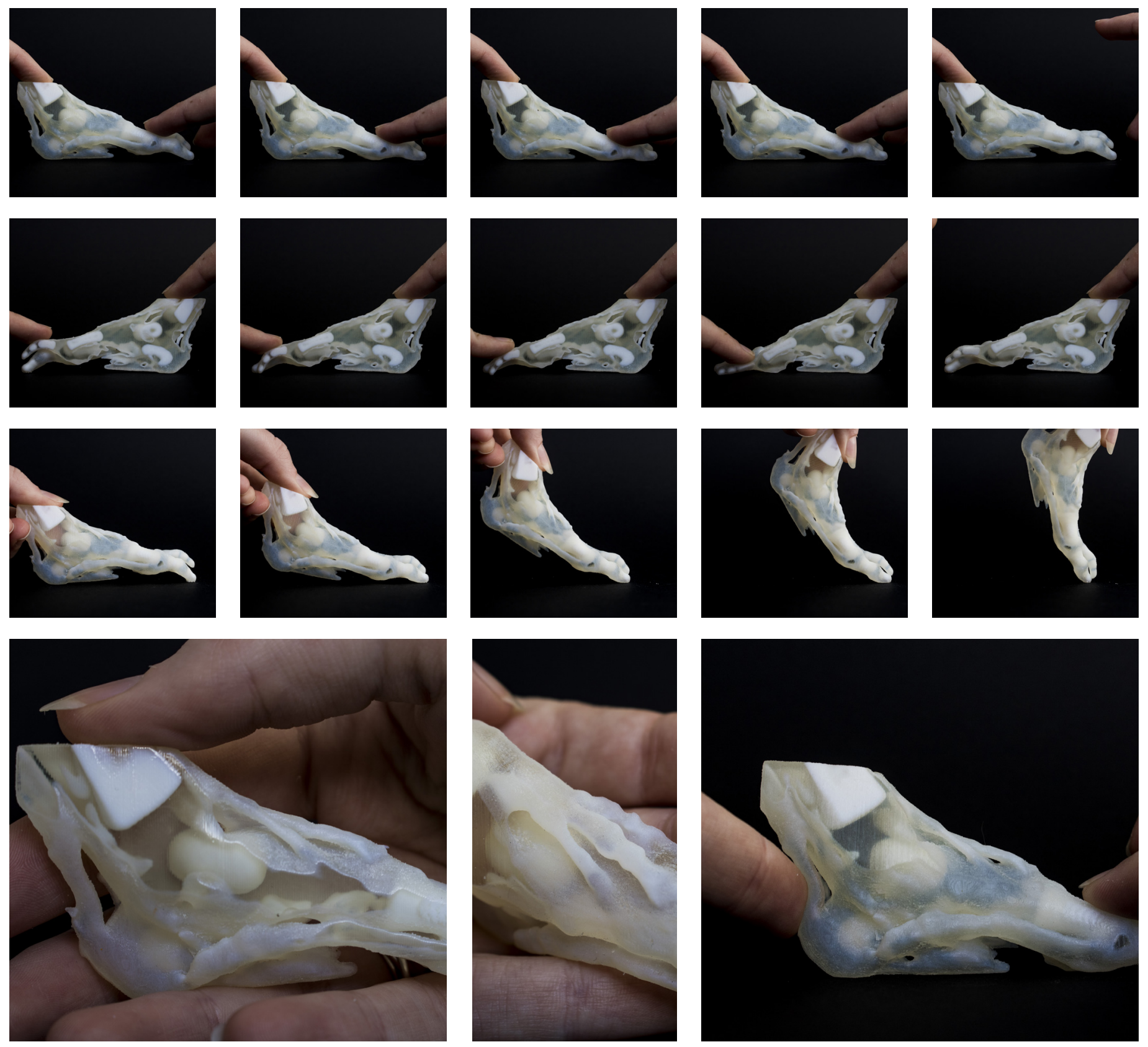

Figure 83. 3D-print \#16.

To translate a CT scan dataset directly into a dynamic 3D-print. Interpolated greyscale CT images were digitally halftoned using black and white. Black was replaced with Agilus $30^{\mathrm{TM}}$ using image thresholding techniques in an image editing, batch processing software.

This 3D-print worked well. The materials blended, 3D-print detail was preserved, and it moved well whilst maintaining structural integrity. 

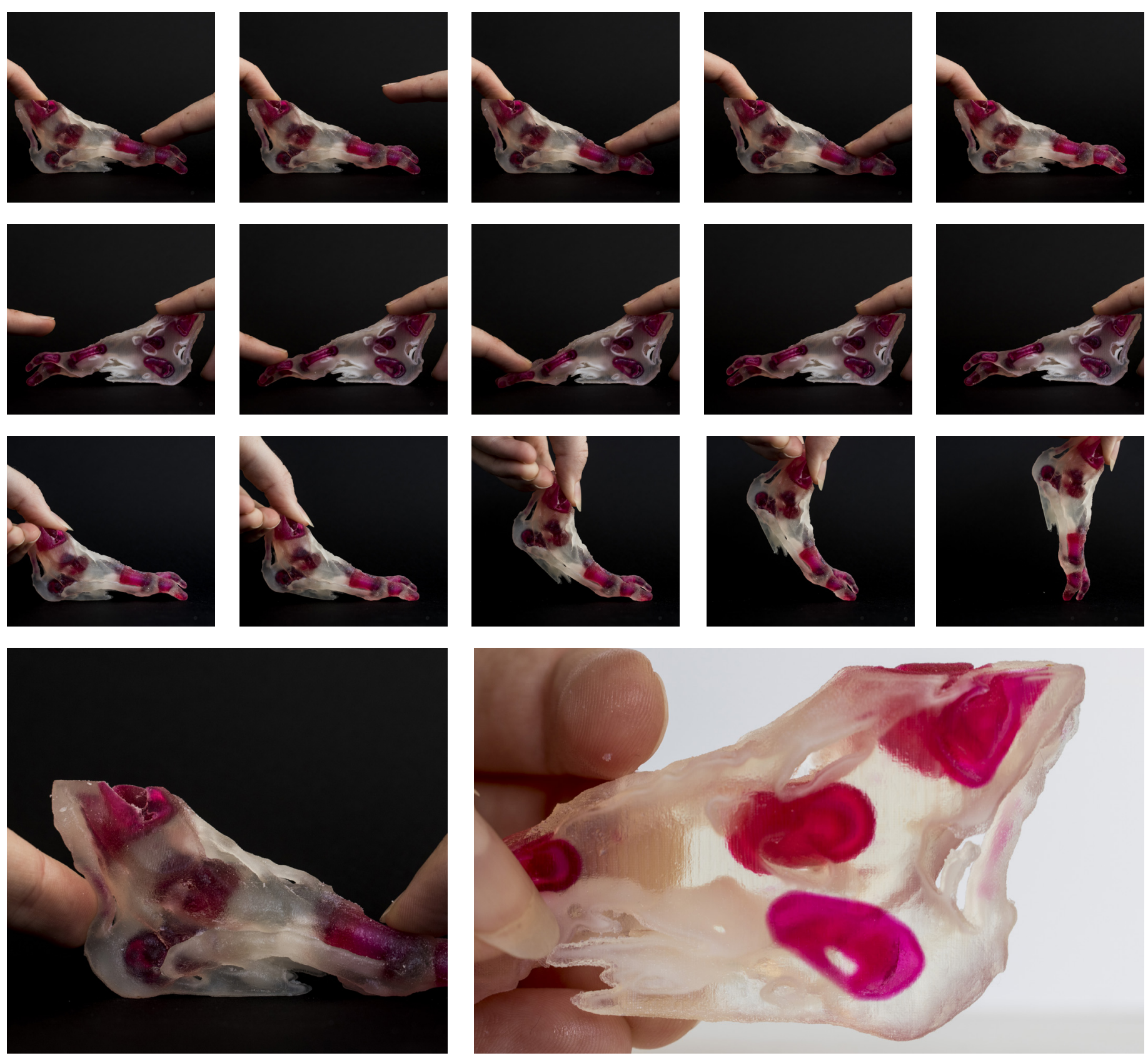

Materials

Figure 84. 3D-print \#17.

Diffusion $40 \%$

To replace greyscale values of a CT dataset with Stratasys J750 ${ }^{\mathrm{TM}}$ 3D-Printer material RGB values in a volumetric reconstruction software. The densest (highest H.U. value) was assigned with VeroMagenta-VTM and the softest area (lowest H.U. value) was as- signed with Agilus $30^{\mathrm{TM}}$. Areas such as inside the bone were assigned with SUP706 ${ }^{\mathrm{TM}}$ to create more realistic bone density.
This 3D-print worked well. It moved realistically and maintained structural integrity. However, the materials did not blend much. The SUP706 ${ }^{\mathrm{TM}}$ created interesting structures within the bone. 

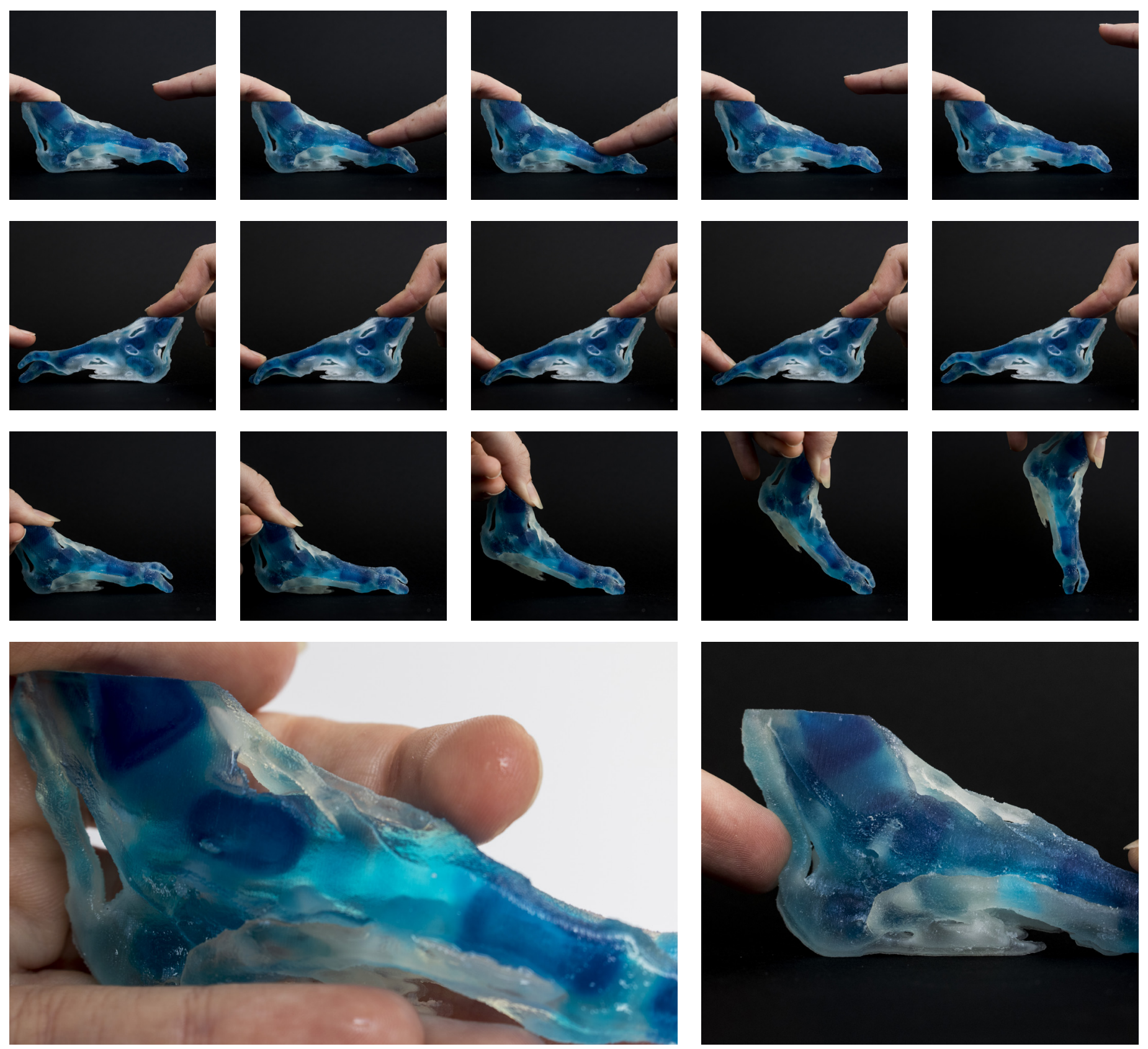

Figure 86. 3D-print \#18 .

To replace greyscale values of a CT dataset with Stratasys J750 ${ }^{\mathrm{TM}}$ 3D-Printer material RGB values in a volumetric reconstruction software. The densest (highest H.U.) value was assigned with VeroCyan-VTM and the softest area (lowest H.U.) was assigned with Agilus30 ${ }^{\mathrm{TM}}$.
The $70 \%$ diffusion of materials meant that materials blended well (created gradients), but a high percentage of VeroCyan-VTM within the Agilus30 $30^{\mathrm{TM}}$ 3D-print stiffened the made the 3D-print more rigid. 

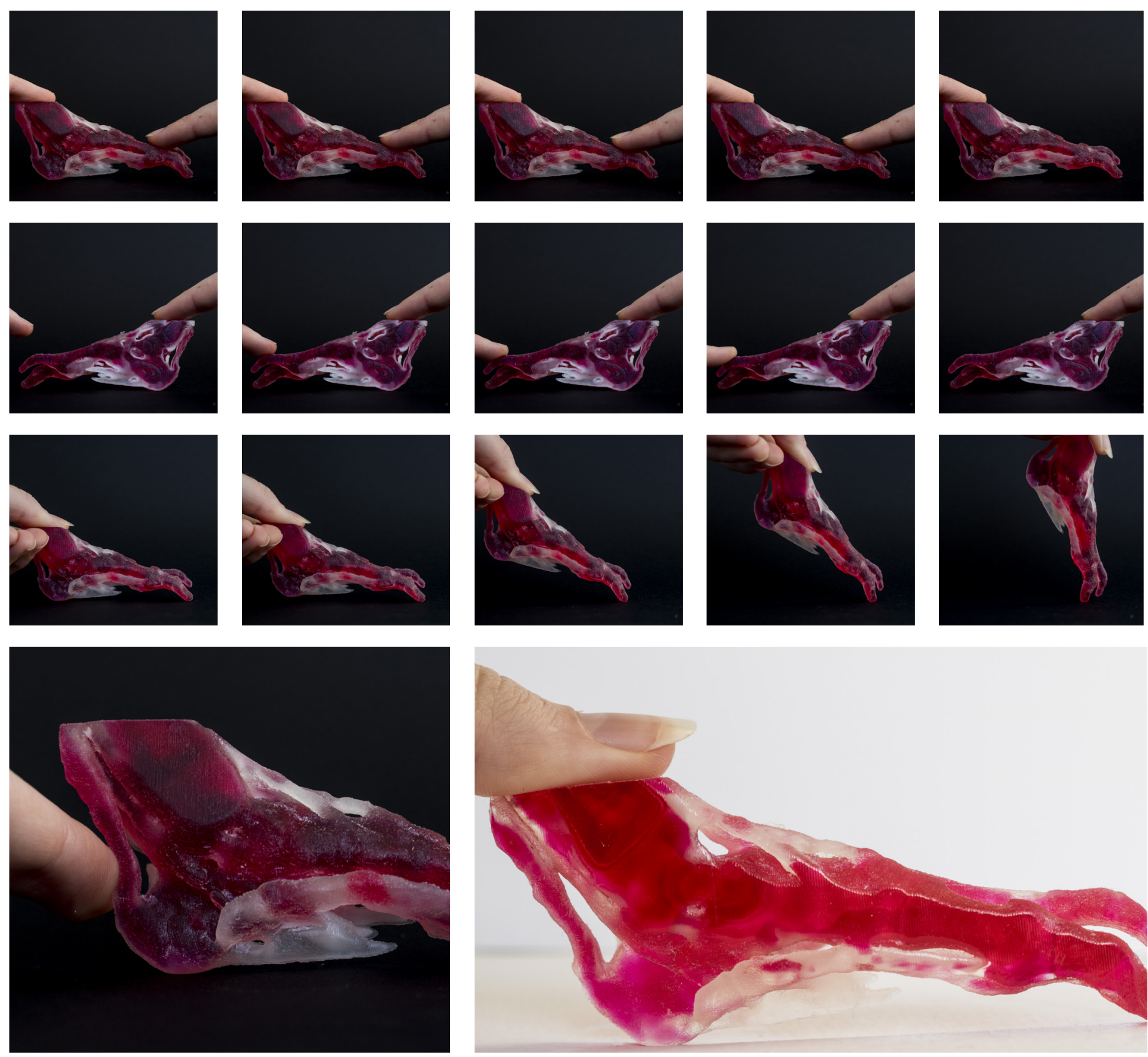

Figure 87. 3D-print \#19.

To replace greyscale values of a CT dataset with Stratasys J750 ${ }^{\mathrm{TM}}$ 3D-Printer material RGB values in a volumetric reconstruction software. The densest (highest H.U.) value was assigned with VeroMagenta-VTM and the softest area (lowest H.U.) was assigned with Agilus30 $30^{\mathrm{TM}}$. The middle H.U. value was assignment with the middle RGB value that lies between the VeroMagenta-VTM RGB value and the Agilus30 ${ }^{\mathrm{TM}}$ RGB value to create a smooth blend between materials.
The combination of VeroMagenta-V $V^{\mathrm{TM}}$ and Agilus $30^{\mathrm{TM}}$ produced the 'orange' middle H.U. value. The dispersion of VeroMagen$\mathrm{ta}-\mathrm{V}^{\mathrm{TM}}$ within the $3 \mathrm{D}$-print made it rigid. 

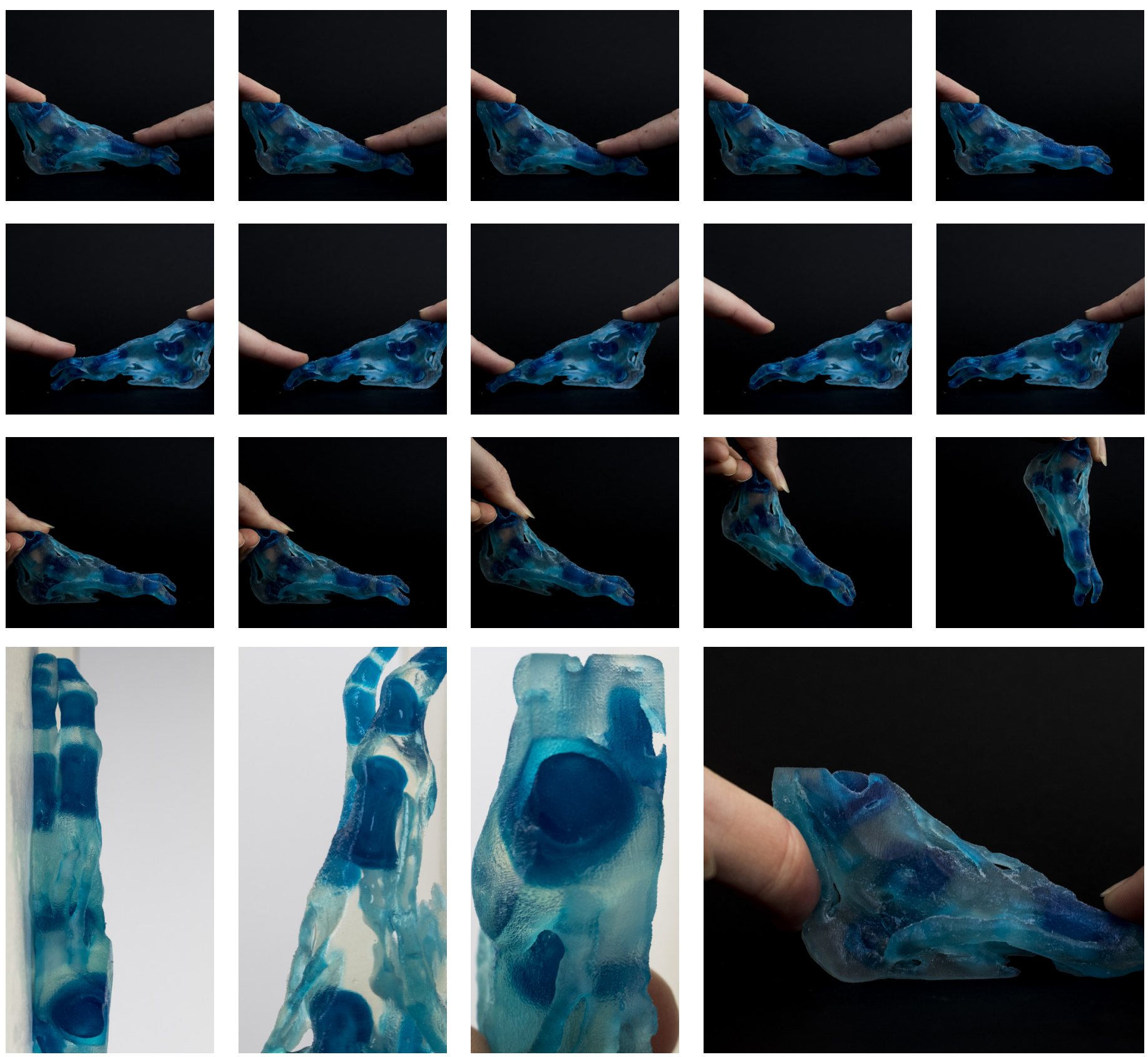

Figure 87. 3D-print \#20.

To translate greyscale CT scan data directly into a dynamic 3D-print without the need to assign materials with an Agilus30 $30^{\mathrm{TM}}$ specific RGB colour. The CT dataset was resliced at 14-micron layer resolution and greyscale files were digitally halftoned in an image processing software.
When adding these files to GradCAD the colour black is picked up as VeroBlackPlus $^{\mathrm{TM}}$.

Even though the image stack was digitally halftoned with black and white pixels, the GrabCAD 3D-Print software picked up multiple greyscale values (based on black and white colour ratios). The lightest greyscale value was assigned with VeroCyan- $-V^{\text {TM }}$ and the rest were assigned with Agilus $30^{\mathrm{TM}}$. 

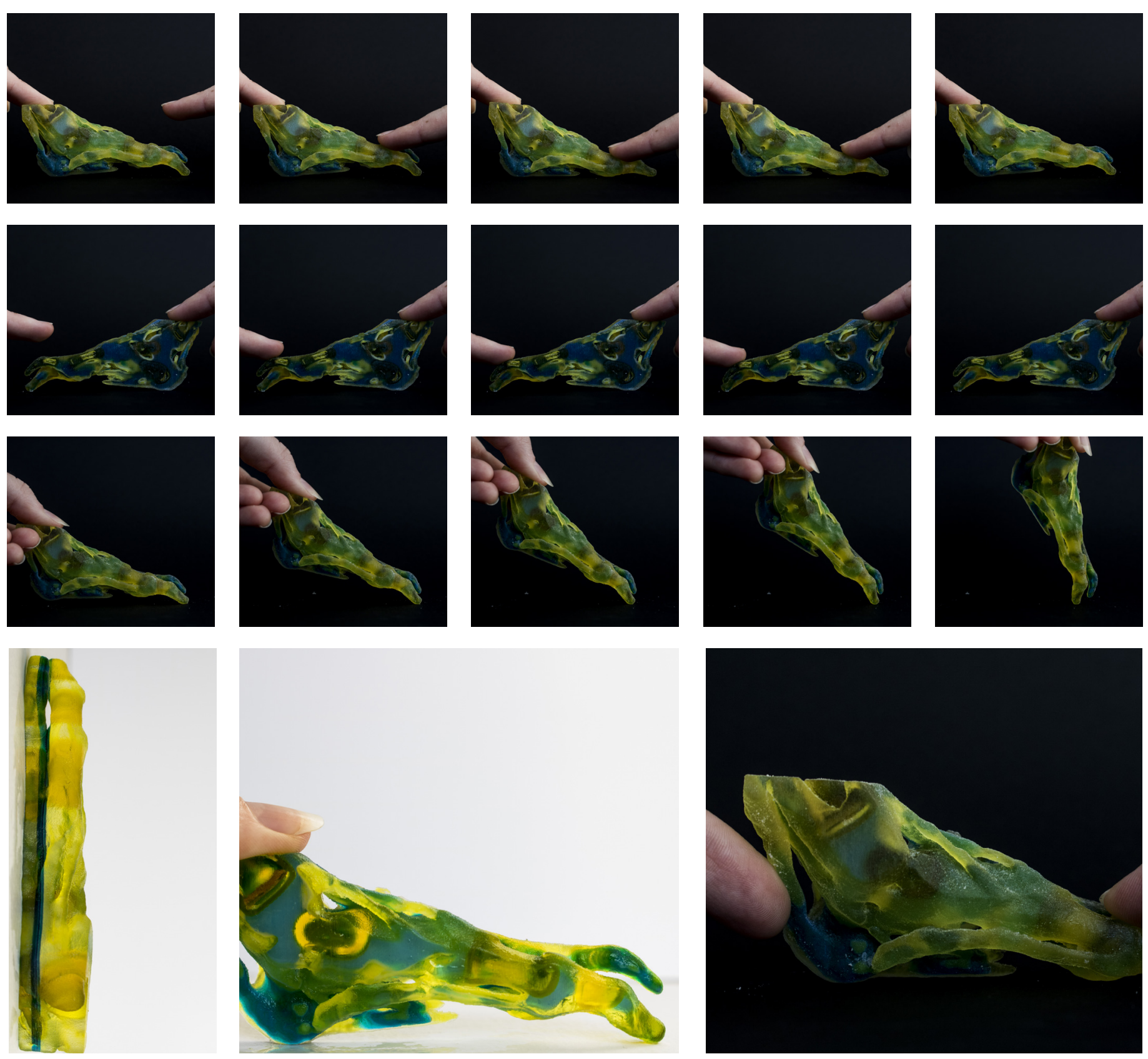

Figure 88. 3D-print \#21.

Same aim as 3D-print \#20.

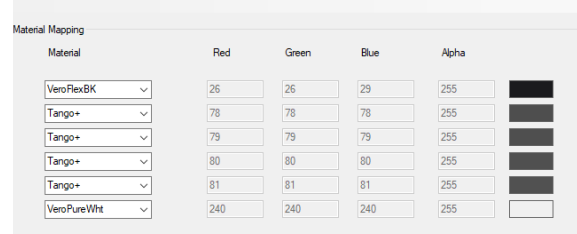

Because of the interesting glitch in Grab$\mathrm{CAD}$, the lightest greyscale value was assigned with Agilus $30^{\mathrm{TM}}$, the middle greyscale value was assigned with VeroYellow- $V^{\mathrm{TM}}$ and the darkest greyscale value was assigned with VeroCyan-VTM .
The 3D-print was particularly interesting because it contained three materials even though the image stacks were dithered with only two pixel colours. However, two Vero $^{\text {TM }}$ colours created a visually complex but rigid 3D-print. 

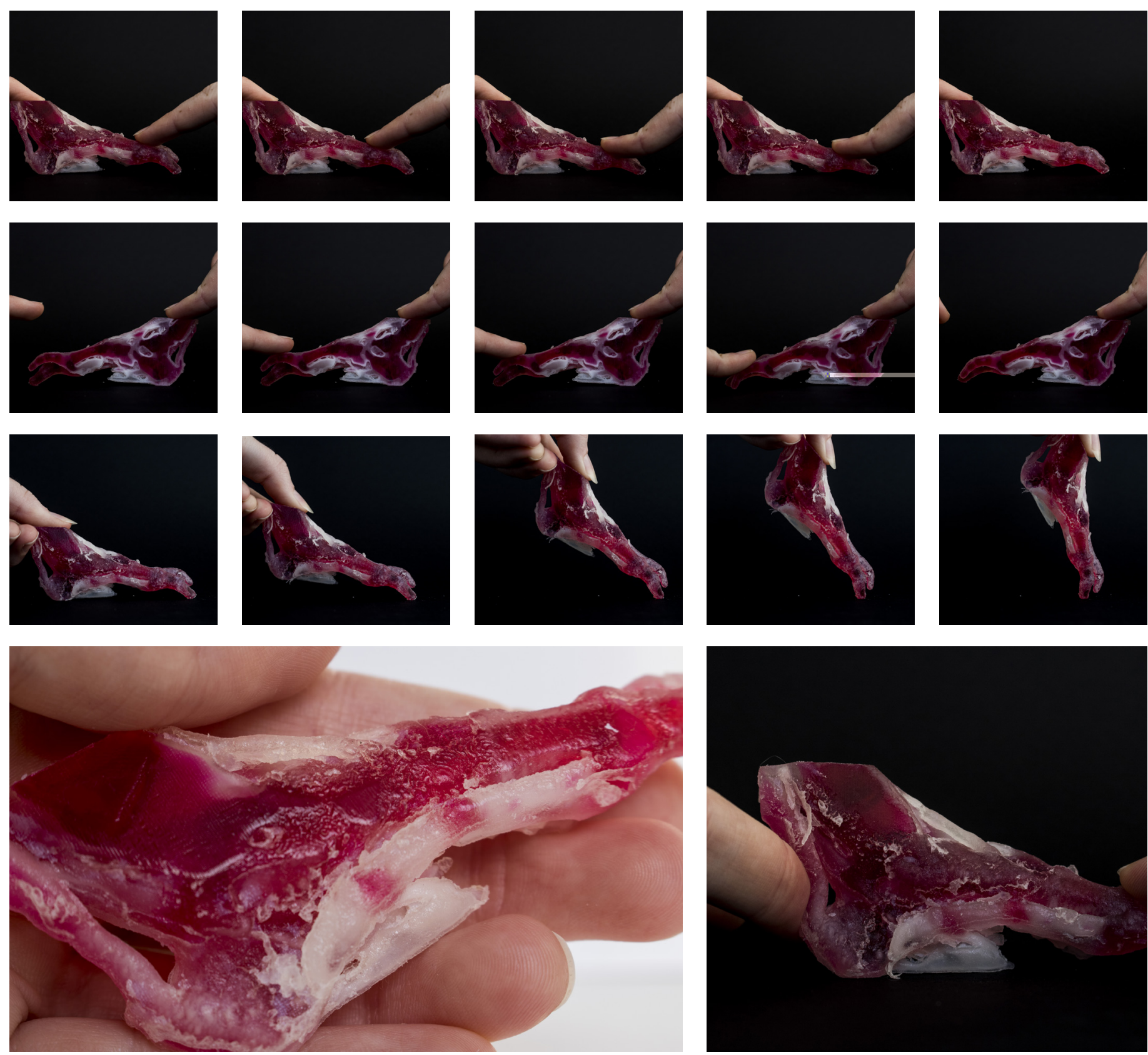

Figure 89. 3D-print \#22 .

To replace greyscale values of a CT dataset with Stratasys J750 TM 3D-Printer material RGB values in a volumetric reconstruction software. The densest (highest H.U.) value was assigned with VeroMagenta-V $V^{\text {TM }}$, the middle HU value was assigned with Agi- lus $30^{\mathrm{TM}}$ and the softest area (lowest H.U.) was assigned with SUP706 ${ }^{\text {TM }}$ to try and create a "softer" (more lifelike) model. An Agilus30 $30^{\text {TM } ~ " s k i n " ~ m u s t ~ b e ~ a d d e d ~ t o ~ t h e s e ~}$ files during the post-processing stage to encapsulate SUP706 ${ }^{\mathrm{TM}}$ within the 3D-print.
The integration of SUP706 ${ }^{\mathrm{TM}}$ made areas of the 3D-print fall apart, creating a surface texture. The areas of the 3D-print with Agilus $30^{\mathrm{TM}}$ remains structural integrity. The 90\% diffusion mean that VeroMagenta-VTM was dispersed throughout the 3D-print, hardening the 3D-print. 

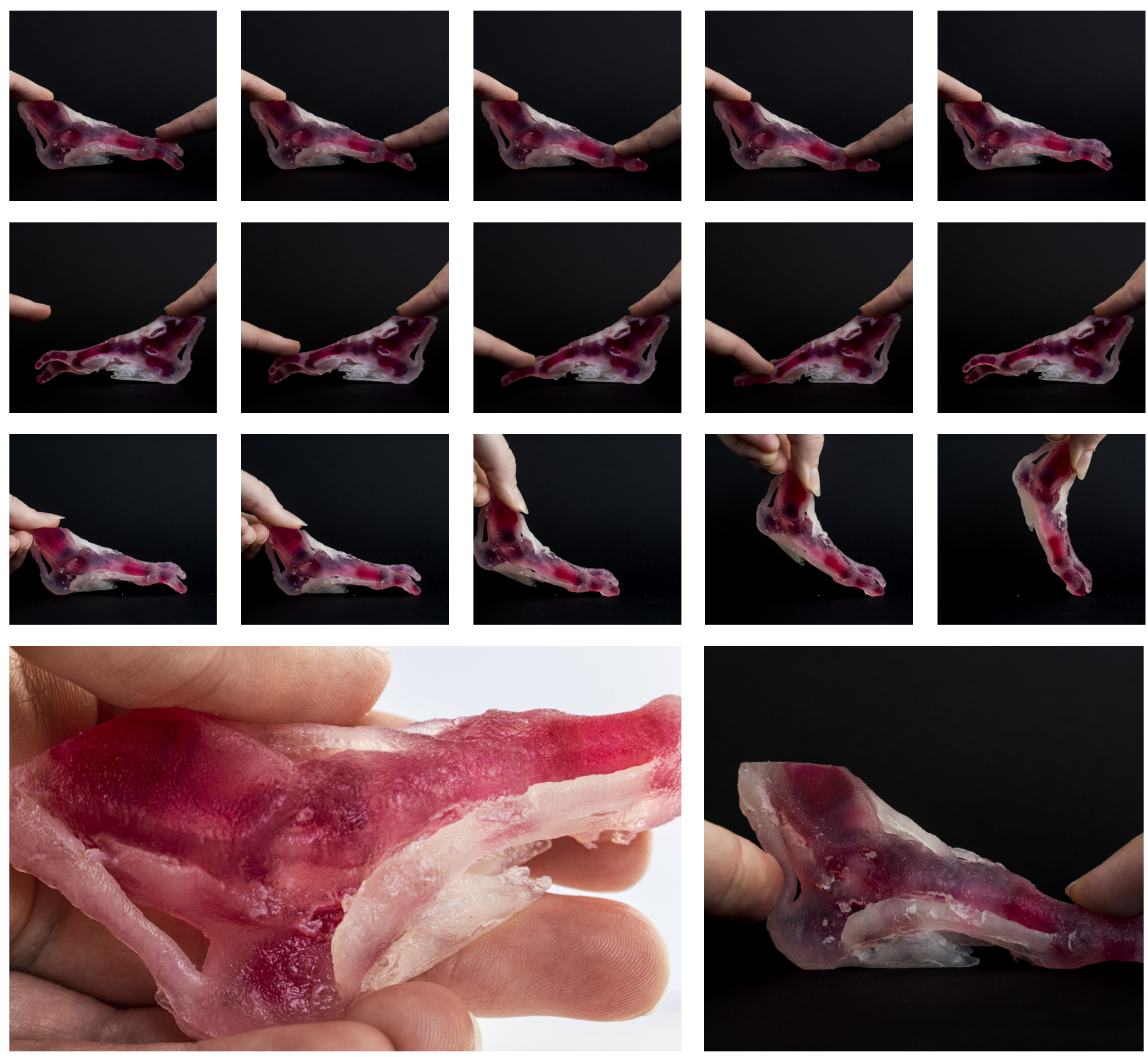

Figure 90. 3D-print \#23.

Materials $\square$ Diffusion 60\%

\#23/Foot 9

This 3D-print is similar to 3D-print \#22. However, the threshold of SUP706 ${ }^{\mathrm{TM}}$ material in the $3 \mathrm{D}$-print was adjusted to include more SUP706 ${ }^{\mathrm{TM}}$ and less Agilus30'TM. A "skin" still must be added to these files during the post-processing stage.
The aim was for the SUP706 ${ }^{\mathrm{TM}}$ to act as a "squishy" fat-like material. 

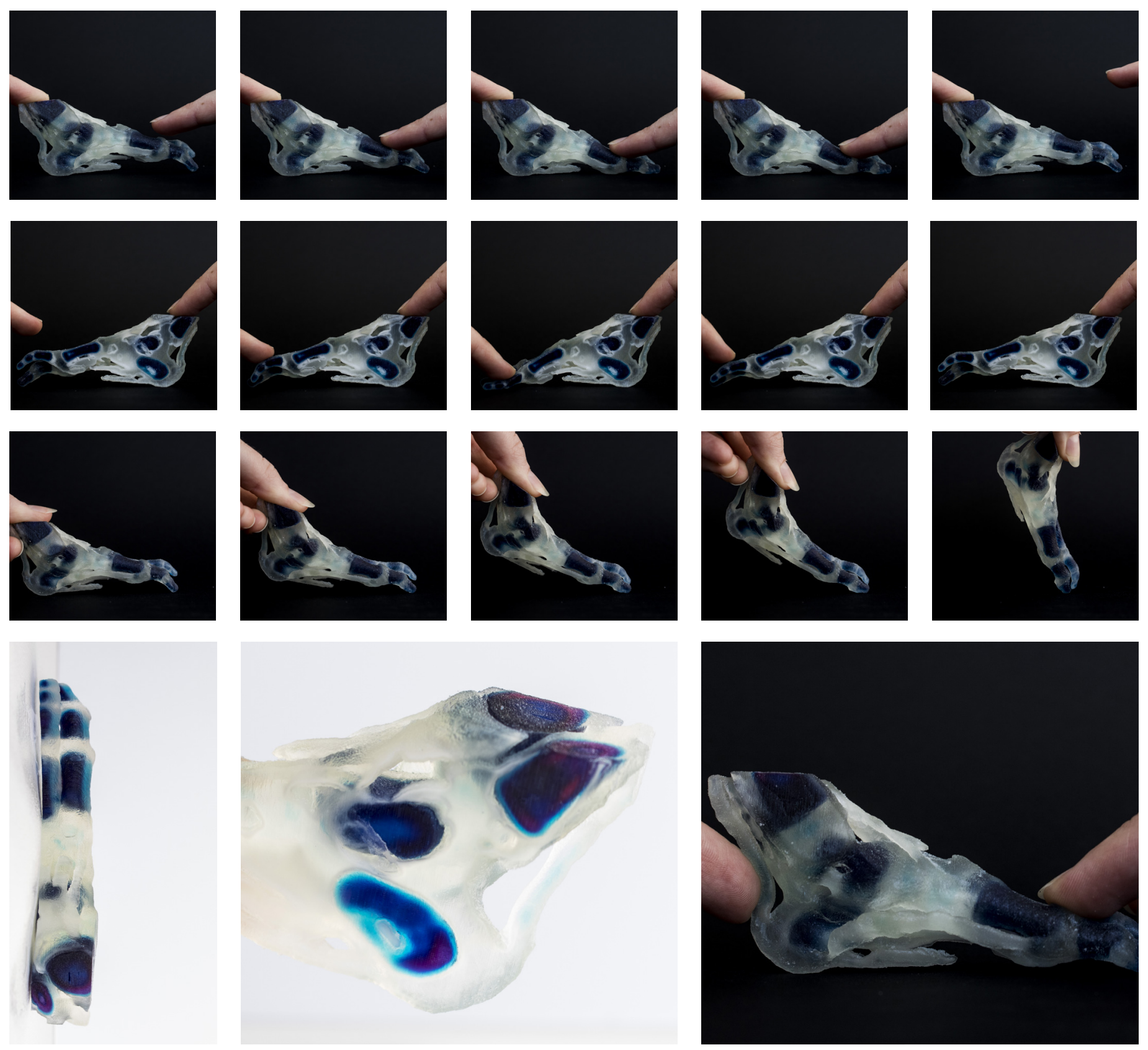

Figure 91. 3D-print \#24.

$\square$ Diffusion $30 \%$

\# 24/Foot 10

To assign two Vero ${ }^{\mathrm{TM}}$ materials (VeroMagenta- $V^{\text {TM }}$ and VeroCyan- $V^{\text {TM}}$ ) to the most dense areas of the CT scan. Then, the soft tissue was assigned with a yellow colour that was slightly lighter than the Agilus30 ${ }^{\mathrm{TM}} \mathrm{RGB}$ value. When these files were post-processed, using digital halftoning algorithms that included the RGB values of Agilus $30^{\mathrm{TM}}$ and SUP706 ${ }^{\mathrm{TM}}$, the two materials blended together. The aim was to create $0.027 \mathrm{~mm}$ pockets of air within the model to produce a lighter, more realistic 3D-print.
SUP706 ${ }^{\mathrm{TM}}$ was dispersed evenly throughout the file mixing with Agilus30 $30^{\mathrm{TM}}$ evenly. This meant the 3D-print was really flexible (feeling more flexible than Agilus30 ${ }^{\mathrm{TM}}$ ) but still maintained structural integrity. Blending two Vero ${ }^{\mathrm{TM}}$ materials resulted in interesting colour gradients within the "bone". 

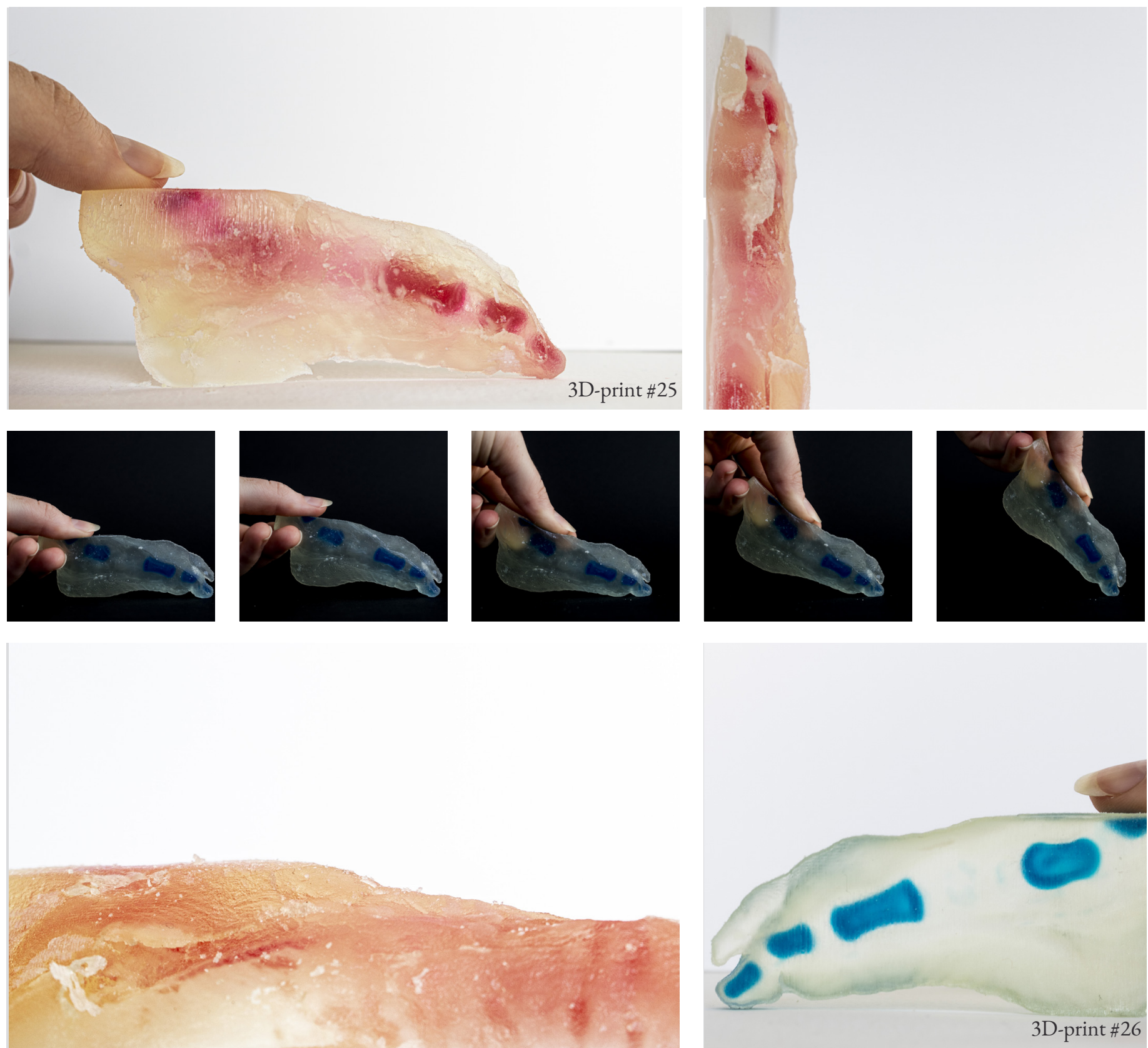

Materials

Figure 92. 3D-prints \#25 and \#26. \#25

\#26

Diffusion 100\%

\#25\&\#26/Foot 11 and Foot 12

\#25 : To produce an area of the foot including the fat and skin. Fat was assigned with SUP706 ${ }^{\mathrm{TM}}$ material. A black value was used as the SUP706 ${ }^{\mathrm{TM}}$ value so that differentiation between black and yellow was more clear. A "skin” stroke was added in post pro- cessing stages to encapsulate the SUP706 ${ }^{\mathrm{TM}}$ within the 3D-print.

\#26: Same aim as 3D-print \#25. However, $3 \mathrm{D}$-print \#26 had more Agilus30 $30^{\mathrm{TM}}$ material dispersed throughout the file.
The skin 3D-printed but was too thin to maintain structural integrity which meant that during the cleaning process, the $3 \mathrm{D}$ print fell apart. However, due to the inclusion of more Agilus30 $30^{\mathrm{TM}}$, 3D-print \#26 maintained more structural integrity than 3D-print \#25. 


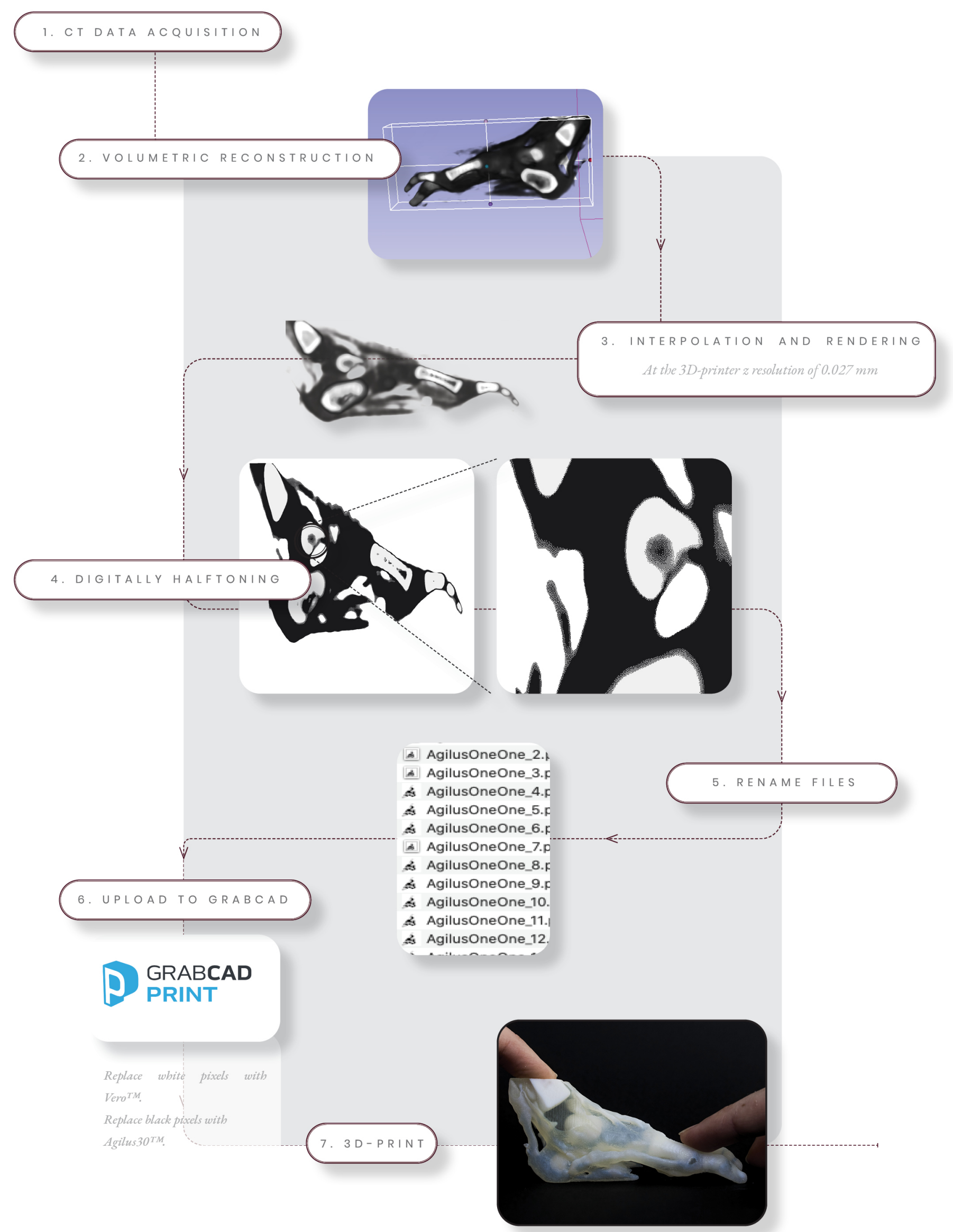

Figure 93. The most successful bitmap-based medical modelling workflow using CT data. 
The most successful 3D-prints were those that rendered out the greyscale images at the $3 \mathrm{D}$-printer native $\mathrm{z}$ resolution and were assigned materials during the digital halftoning and batch processing stage of the process (such as $3 \mathrm{D}$ print \#16). The material combinations that were the most effective were using Agilus $30^{\mathrm{TM}}$ in combination with Vero ${ }^{\mathrm{TM}}$ materials. Generally, including SUP706 ${ }^{\mathrm{TM}}$ within a $3 \mathrm{D}$-print has the potential to compromise the structural integrity of the model.

After determining the most successful bitmap-based 3D-printing workflow to produce dynamic anatomical models, a decision was made to 3D-print a 1:1 scale neonatal leg, starting with the ankle, knee, and hip joints. Including a variety of joints presented new challenges and helped to highlight the advantages and disadvantages of bitmap-based 3D-printing for medical modelling purposes.

The focussed and final prototyping during Case Study 2 was informed by discoveries made in initial design experiments and followed the same digital workflow. 

4.1 .5

Design Refinement

Left:

Figure 94. Bitmap-based 3D-printed neonatal feet. (A) Skin and fat included. (B) No skin and fat. 


\section{Rendering in the Correct Scale}

\section{Obstacle}

Exporting image stacks using medical imaging software can cause scaling errors. Determining the correct scale occurred by selecting a small area of anatomy, exporting this area of anatomy as cross-sectional bitmap image files, and evaluating which workflow resulted in a correct 1:1 medical model. The area of anatomy chosen for testing was a thin cross-section of the leg. The correct test should have a thickness $18.36 \mathrm{~mm}$, the same measurement as the 1:1 scale volumetric visualisation.

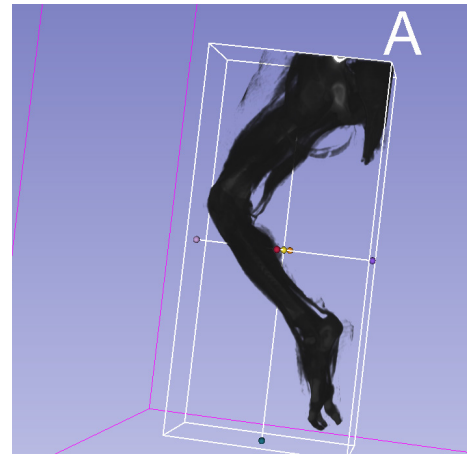

Figure 95. Virtual reconstruction of neonatal leg.

Table 3. Testing File Exportation settings

TEST

X RESOLUTION

Y RESOLUTION

LAYER THICKNESS

S C A LE

POST-PROCESSING

CORRECT SCALE
300 DPI

300 DPI

.014

$.5 x$

increase image size

by $400 \%$

None

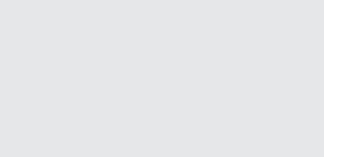

655 images

$17.6 \mathrm{~mm}$
TES T 2

TES T 3

$150 \mathrm{DPI}$

$150 \mathrm{DPI}$

.027

$1 x$

increase image size

by $200 \%$

change to 300 DPI

680 images

$18.36 \mathrm{~mm}$ 


\section{D-Printing Foot, Knee and Hip Joints}

Once the correct scale was established, the joint files (foot, knee and hip) were exported and 3D-printed. If joint files are exported separately, there is a risk of the files not being consistent. The 3D-printed leg was exported and then cropped after exportation during the digital halftoning automated batch processing. A total of 2,282 images were processed to complete a bitmap-based 3D-print of a neonatal leg (without skin and fat).

To create the 1:1 full scale test 3D-prints of a neonatal limb joints, the bitmap images were scaled into the 3D-printer's native $\mathrm{X}$ and $\mathrm{Y}$ resolutions and digital halftoning algorithms were used to create pixel combinations using the primary 3D-printer materials of VeroMagenta-V $\mathrm{V}^{\mathrm{TM}}$ and Agilus $30^{\mathrm{TM}}$ with a $70 \%$ pixel diffusion ratio. Each pixel holds material specification information for the 3Dprinter and the pixel combinations (ratios) and the overall aim was to reflect the greyscale attenuation values of the CT scan data in a physical model. The ratio of soft (Agilus $30^{\mathrm{TM}}$ ) material to hard $\left(\right.$ Vero $\left.^{\mathrm{TM}}\right)$ material determines the greyscale value.

Images were imported into the GrabCAD Voxel Print Utility and were exported as a GrabCAD Voxel Files (.GCVF file). A total of 2,282 images were processed to complete the full leg (without fat and skin), 1,494 images completed the foot, 2,019 images completed the knee and 2,232 images completed the hip.

Approximately 185 images completed the cross-sectional tests of each joint.

1,677 images completed the neonatal foot with bone, soft tissue, fat and skin.

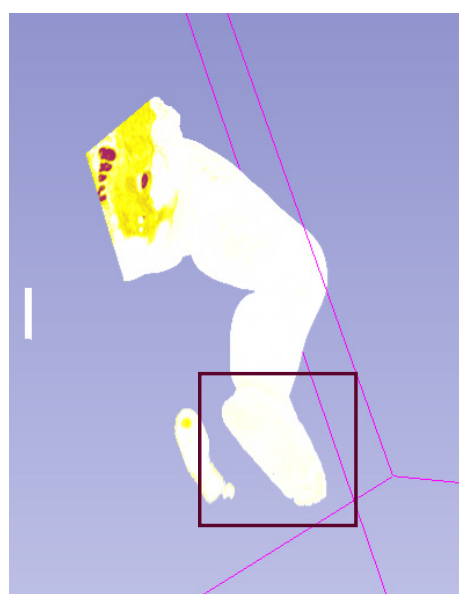

Figure 96. Virtual reconstruction of neonatal leg (with skin and fat).

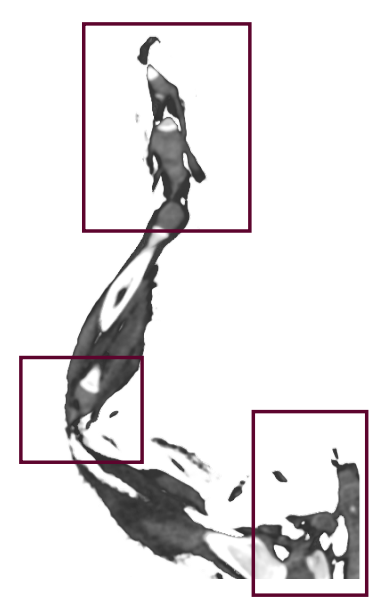

Figure 97. Cropped regions for joint 3D-prints. 


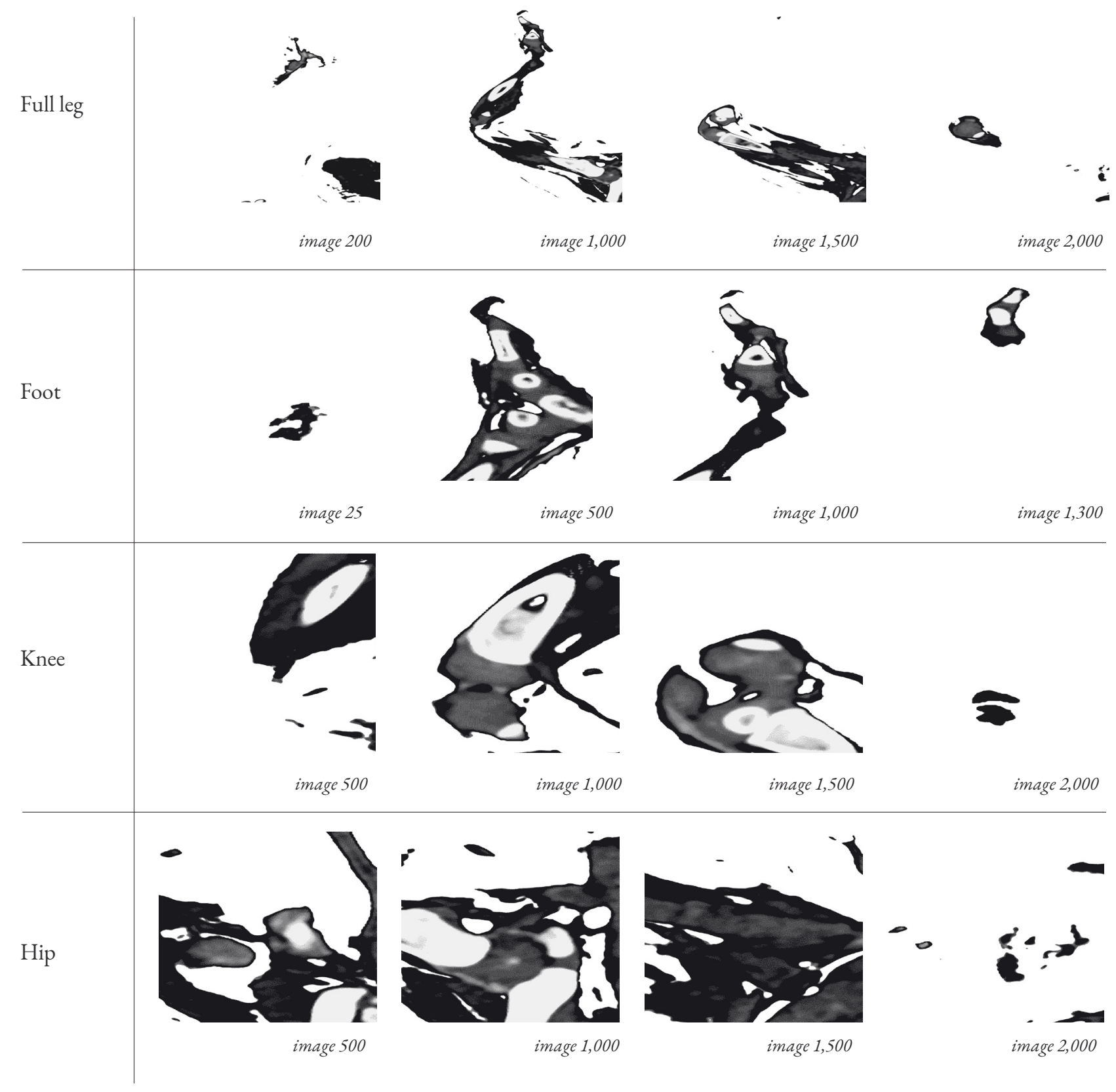



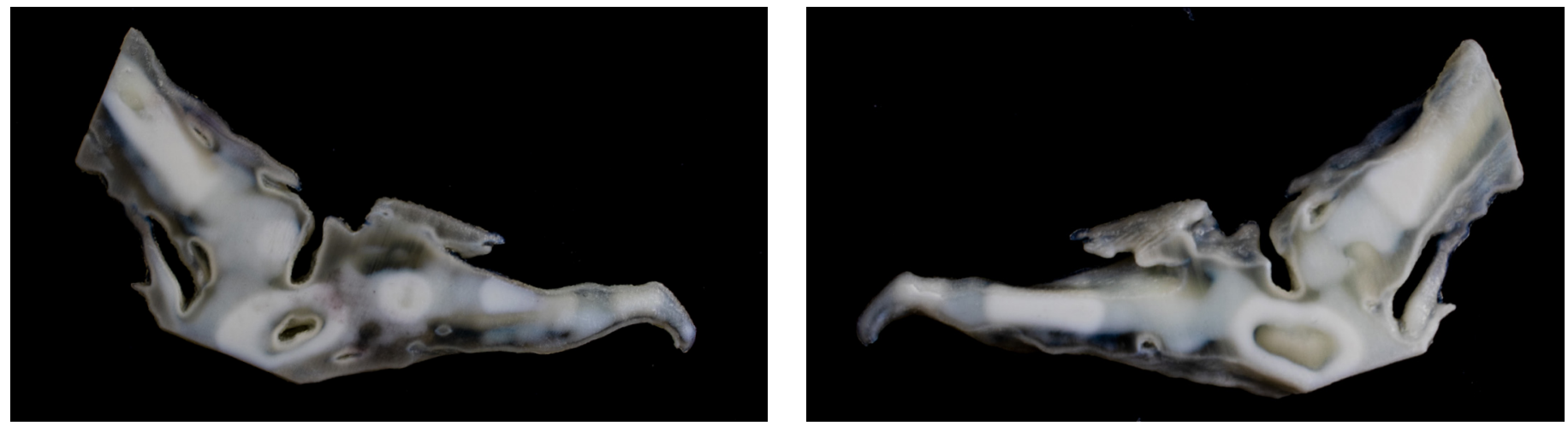

\# 27 / Foot (cross-section)
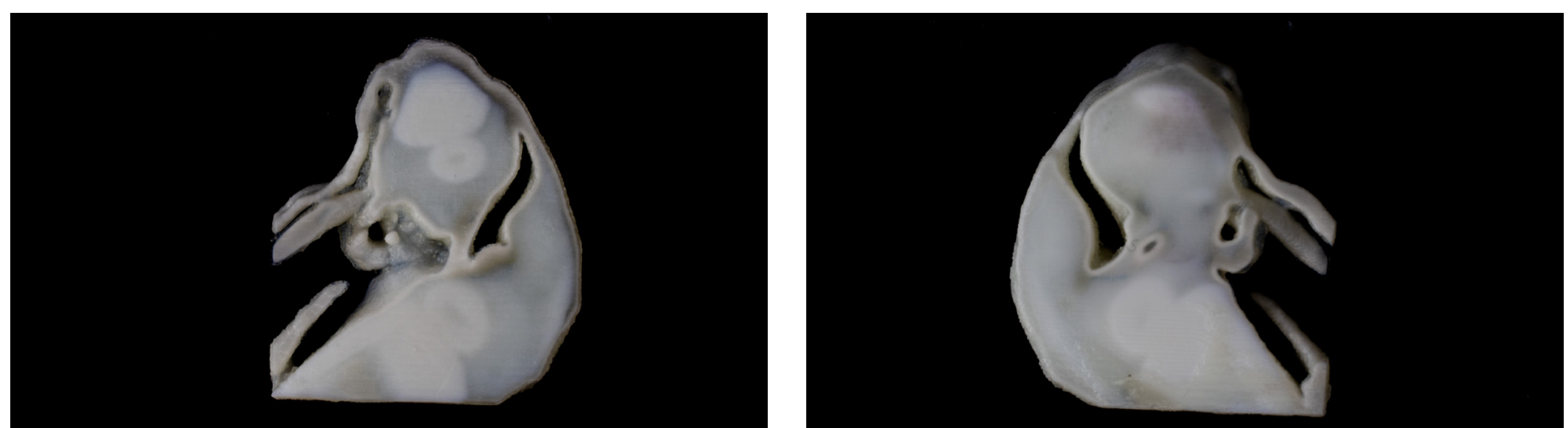

\# 28 / Kne e (cross-section)
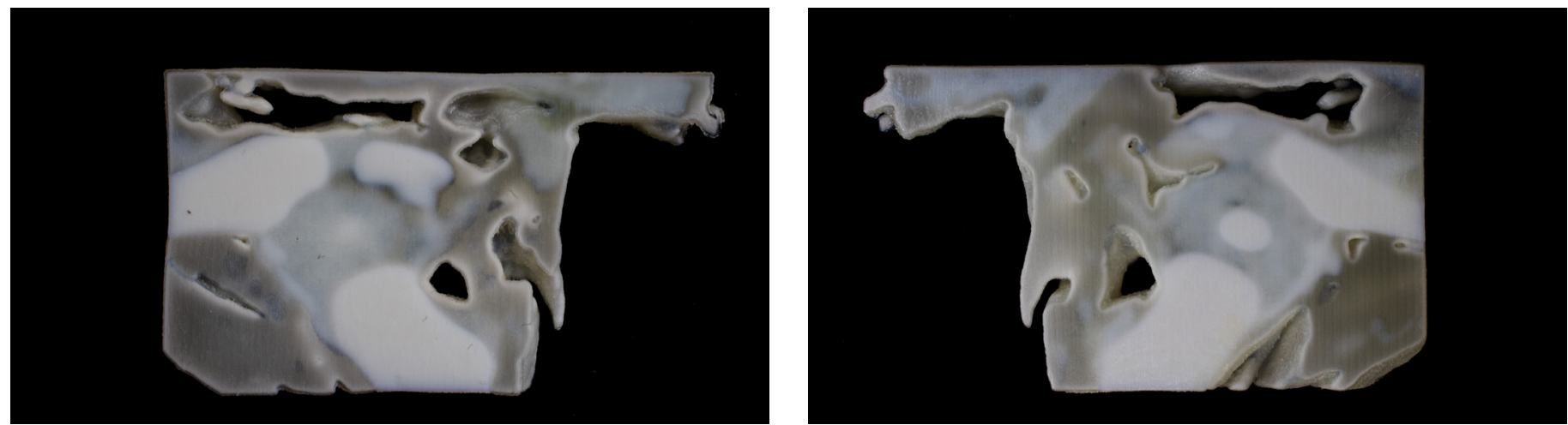

\# 29 / Hip (cross-section)

Figure 99. 3D-prints \#27, \#28 and \#29 (cross-sections of joints).

To 3D-print cross-sections of the joints to test the exportation settings and 3D-print quality before 3D-printing 1:1 scale joints.
The cross-sectional 3D-prints were successful. The material gradated well, they were the correct scale and they bent and moved as hoped. 


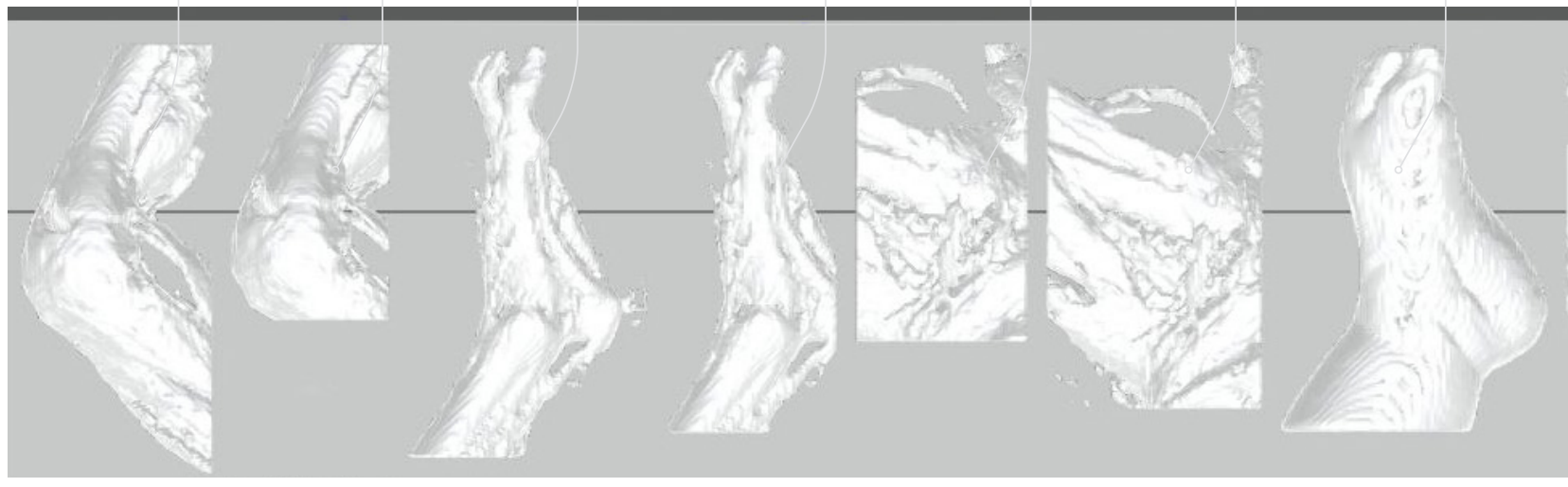

Figure 100. GrabCAD Print tray (neonatal joints).

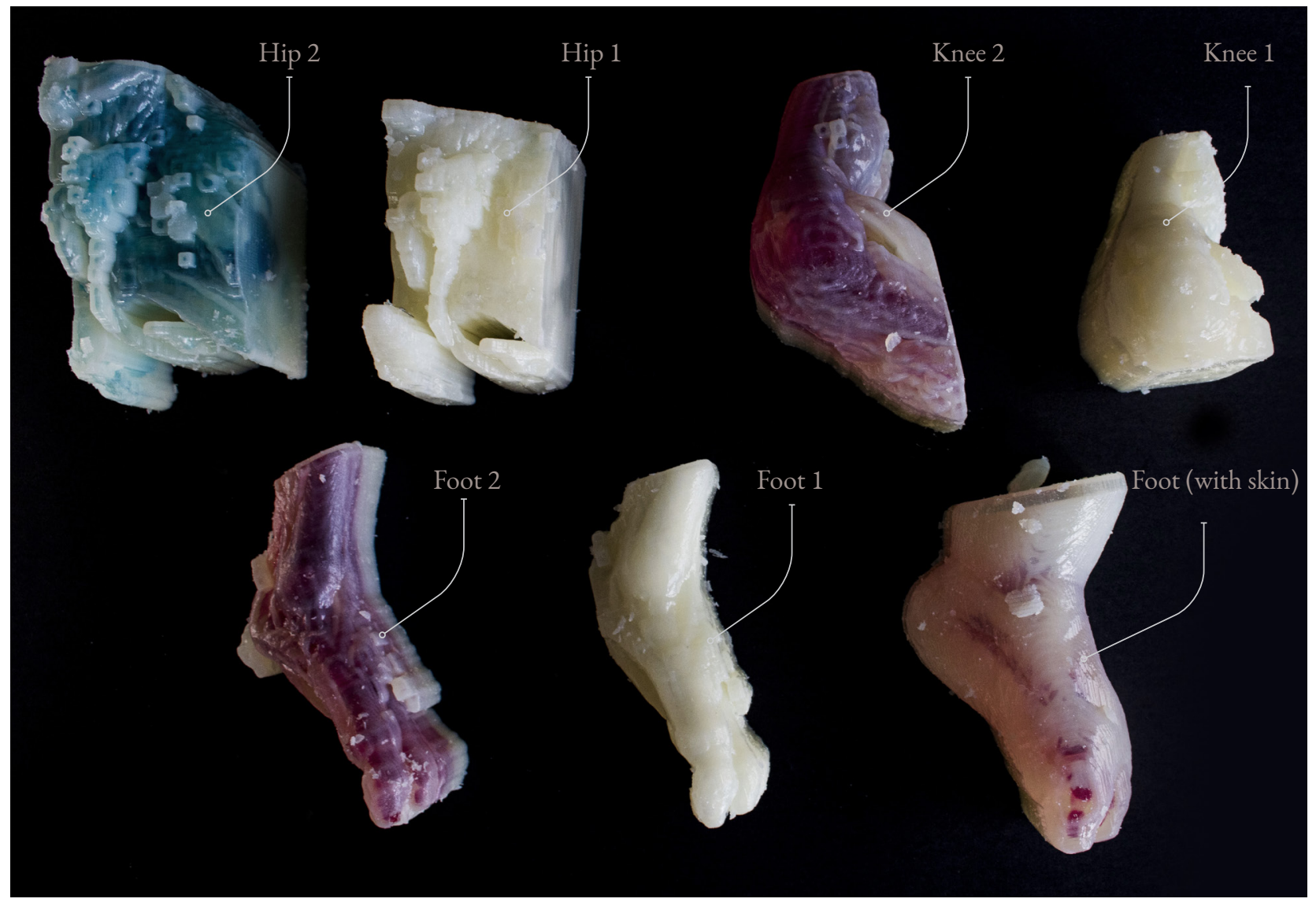

Figure 101. 3D-printed joints coated in SUP706 ${ }^{\mathrm{TM}}$. 


\section{D-printing Full 1:1 Scale Models of a Neonatal Foot, Knee and Hip}

Two versions of the full leg were produced from the exported interpolated data. One version of the leg was dithered at $70 \%$ diffusion (like the cross-sectional 3D-prints presented in Figure 99.) and the other at 30\% diffusion (based on results from the cross-sectional 3D-prints).

For differentiation purposes:

The 3D-printed joints (foot, knee, and hip) that were dithered at 70\% diffusion used the materials VeroPureWhite ${ }^{\mathrm{TM}}$ and Agilus $30^{\mathrm{TM}}$.

The 3D-printed joints (foot, knee, and hip) that were dithered at 30\% diffusion used the materials VeroMagenta-V $V^{\mathrm{TM}}$, VeroCyan-V $V^{\mathrm{TM}}$ and Agilus30 $30^{\mathrm{TM}}$. 

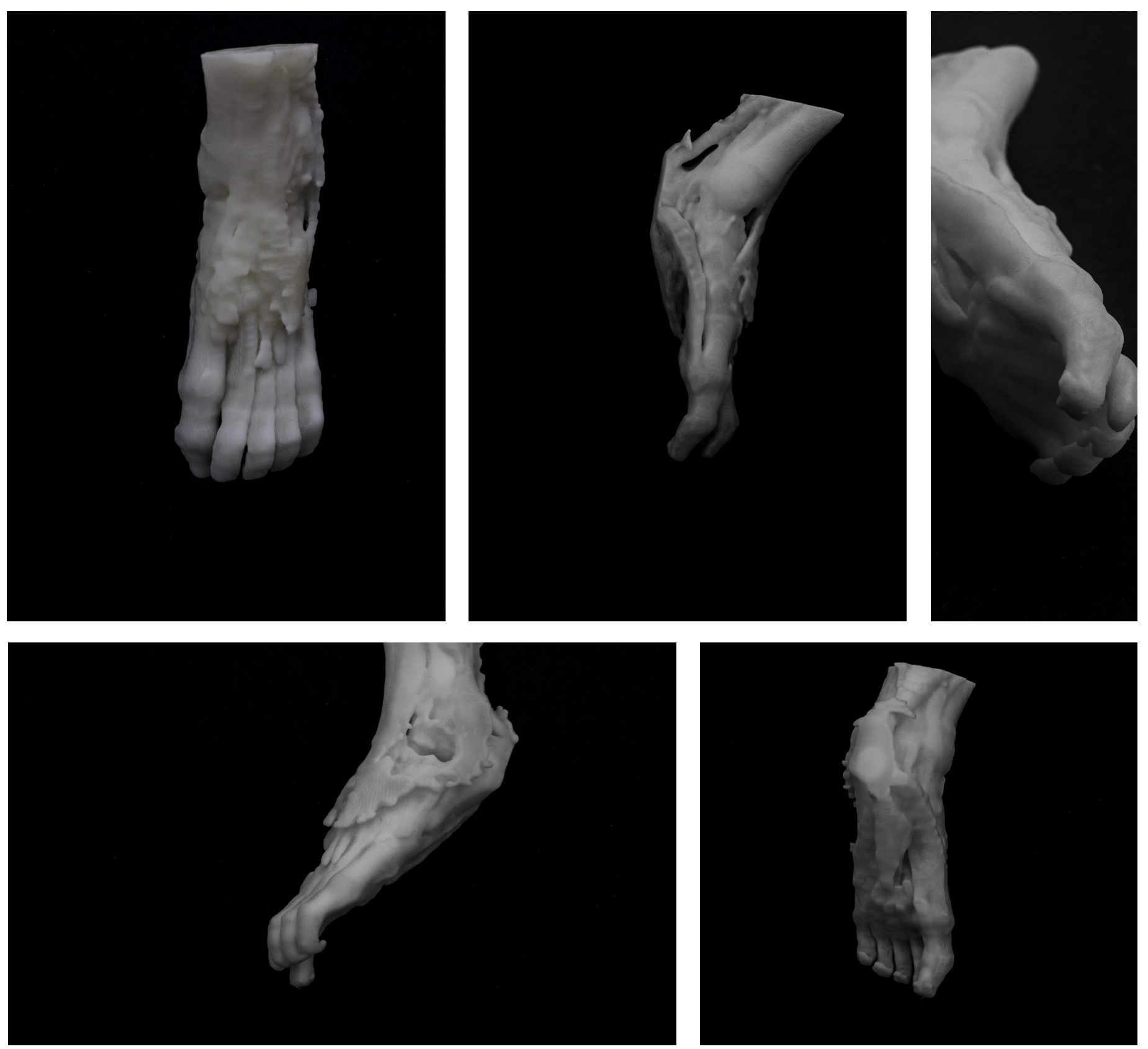

Materials

Figure 102. 3D-print \#30.

A i m

To use a bitmap-based 3D-printing workflow to 3D-print a full 1:1 scale neonatal foot using $70 \%$ diffusion.
Discovery

The 3D-printed foot preserved a significant amount of anatomical detailed and photographed well. However, the 3D-print remained quite rigid. 

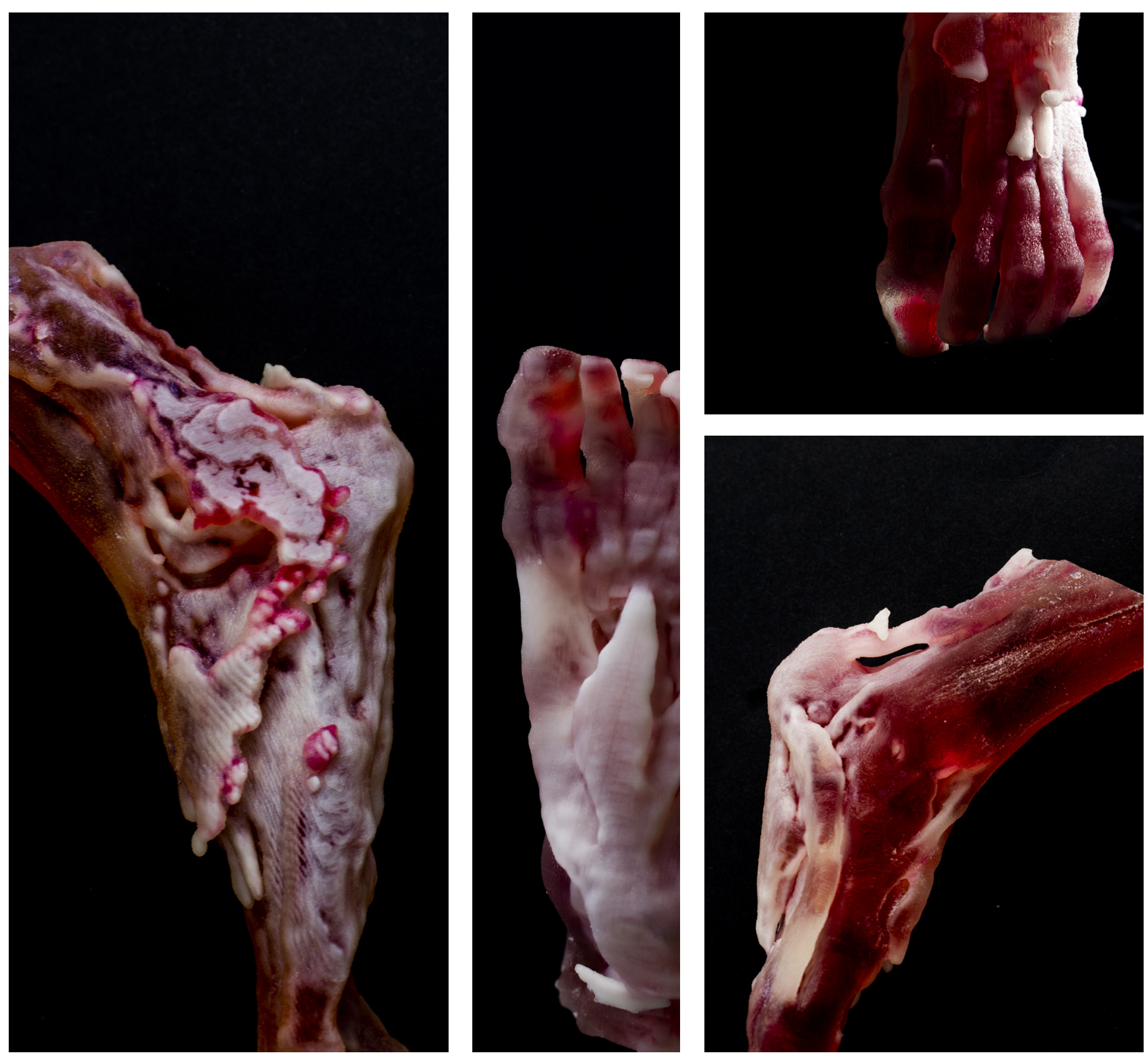

Figure 103. 3D-print \#31. Diffusion 30\%

To use a bitmap-based 3D-printing workflow to 3D-print a full 1:1 scale neonatal foot using 30\% diffusion and coloured material.
The 3D-printed foot preserved anatomical detail and photographed well. The 3D-print was substantially less rigid than 3D-print \#40 and the visual gradients created using VeroMagenta-VTM meant that more anatomical details were captured visually. 

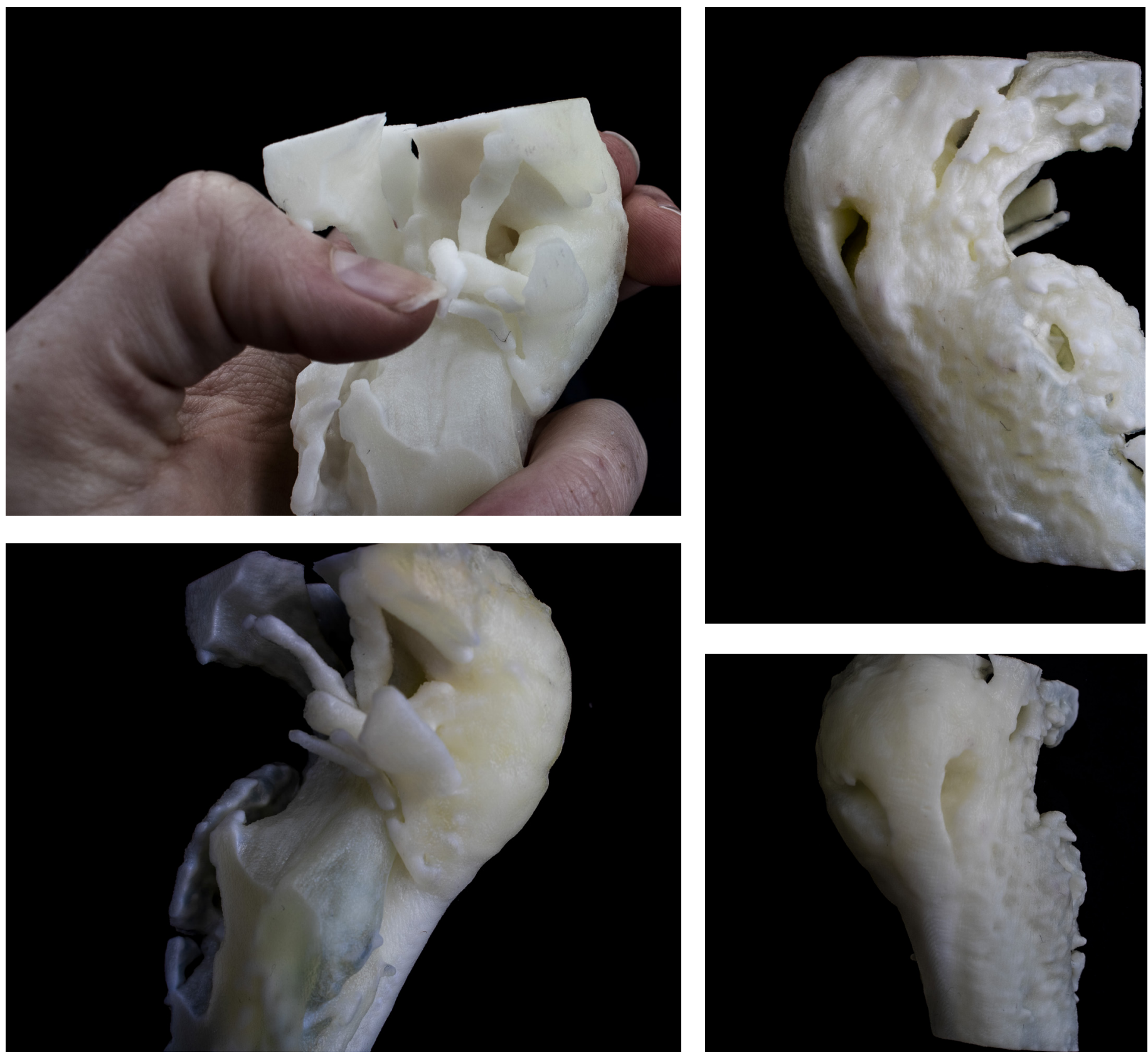

A i m

To use a bitmap-based 3D-printing workflow to 3D-print a full 1:1 scale neonatal knee using $70 \%$ diffusion.
The 3D-printed knee preserved a significant amount of anatomical detail and photographed well. The joint remained rigid but the ligaments had dynamic movement, exposing some interesting, small anatomical details. 

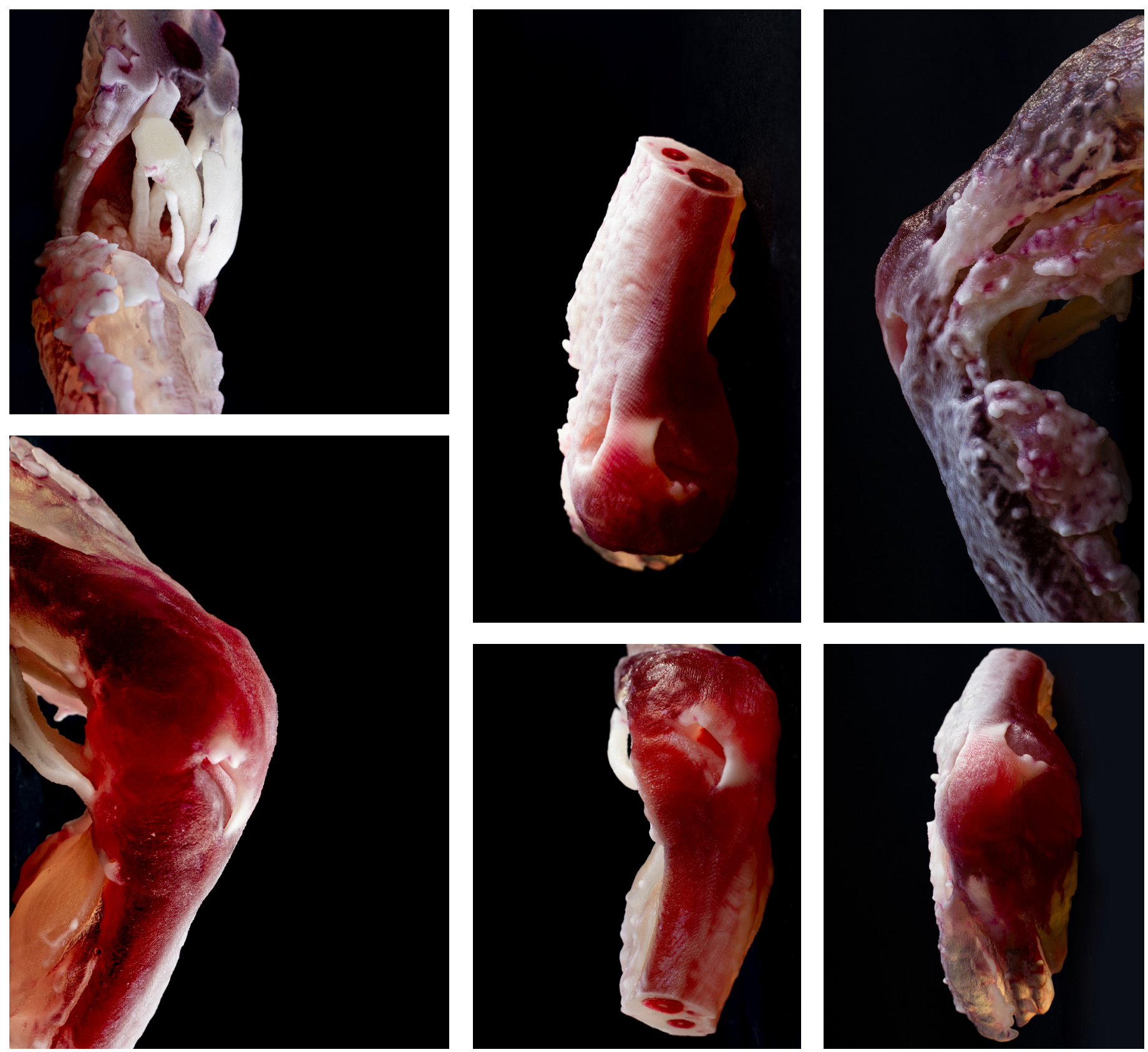

Figure 105. 3D-print \#33

Diffusion 30\%

\# $331 \mathrm{knee}$

To use a bitmap-based 3D-printing workflow to 3D-print a full 1:1 scale neonatal knee using 30\% diffusion and coloured material.
The 3D-printed knee preserved anatomical anatomical details were captured visually. detailed and photographed well. The 3D- The photographs captured the texture on print was substantially less rigid than $3 \mathrm{D}$ - the soft tissue and appeared lifelike in phoprint \#42 and the visual gradients created tography. using VeroMagenta-V ${ }^{\mathrm{TM}}$ meant that more 

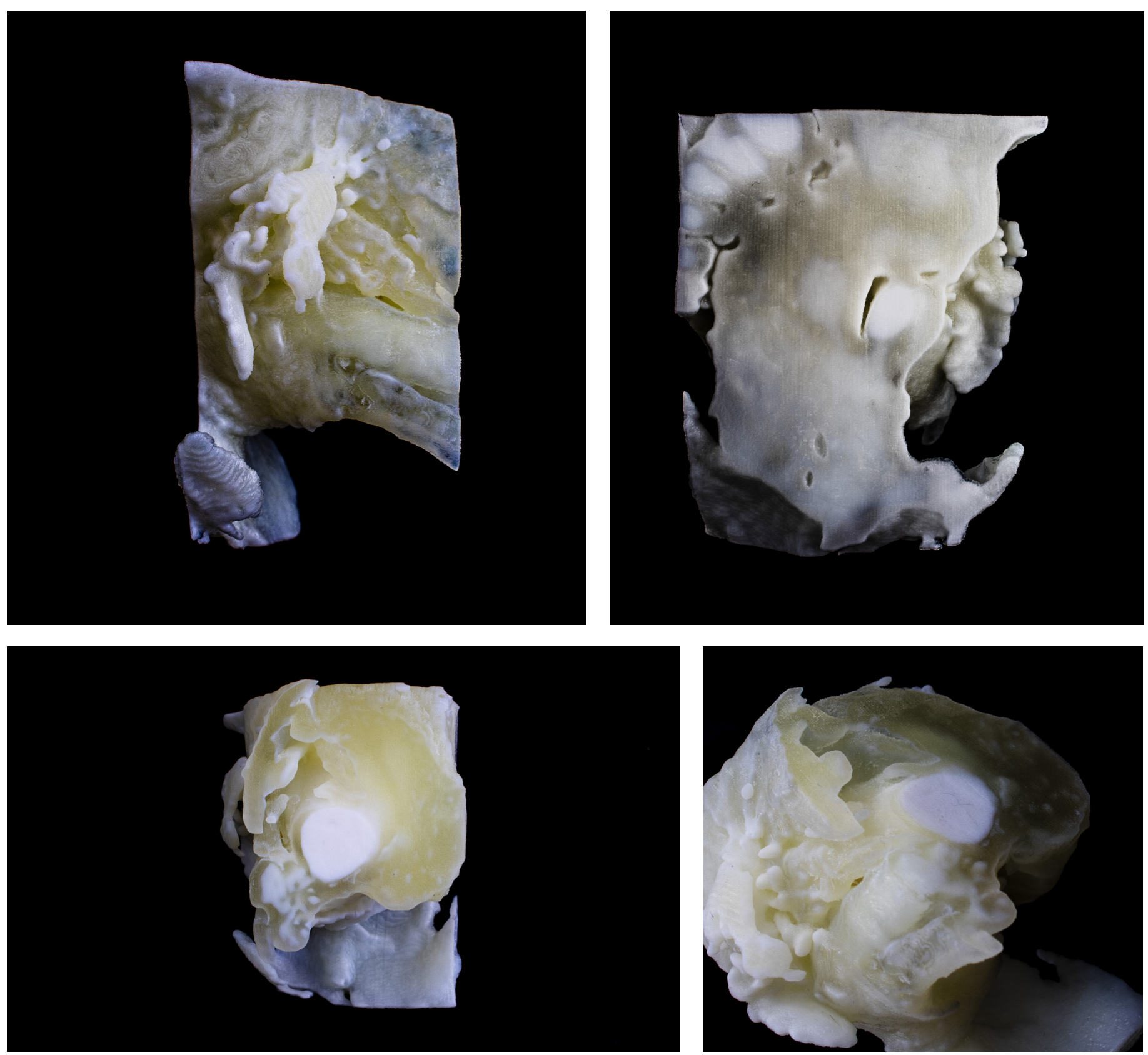

Figure 106. 3D-print \#34.

To use a bitmap-based 3D-printing workflow to 3D-print a full 1:1 scale neonatal hip using $70 \%$ diffusion.
The 3D-printed hip preserved a significant amount of anatomical detail and photographed well. The joint remained rigid, but the soft tissue surrounding the bone structure was soft. An interesting feature of this 3D-print was the spine (bone). 

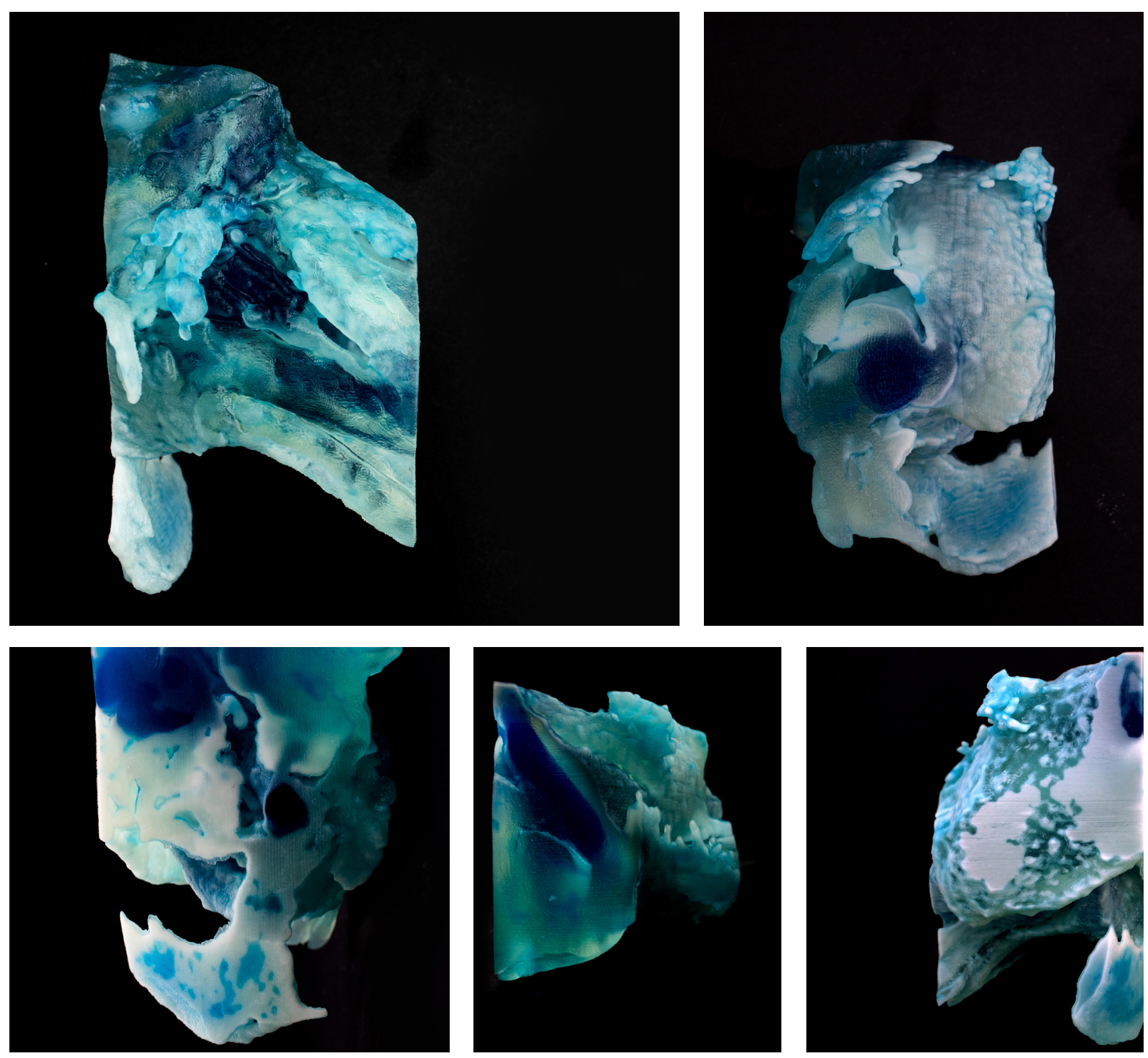

Figure 107. 3D-print \#35.

Diffusion 30\%

\# $35 / \mathrm{Hip}_{2}$

To use a bitmap-based 3D-printing workflow to 3D-print a full 1:1 scale neonatal hip using 30\% diffusion and coloured material.
The 3D-printed hip preserved anatomical cal details were captured visually. The visual detail and photographed well. The 3D-print qualities created through blending VeroCywas substantially less rigid than 3D-print an- $\mathrm{V}^{\mathrm{TM}}$ were interesting (not lifelike, but \#4 and the visual gradients created using visually interesting, almost like a candy or VeroCyan-V ${ }^{\mathrm{TM}}$ meant that more anatomi- ocean shell). 

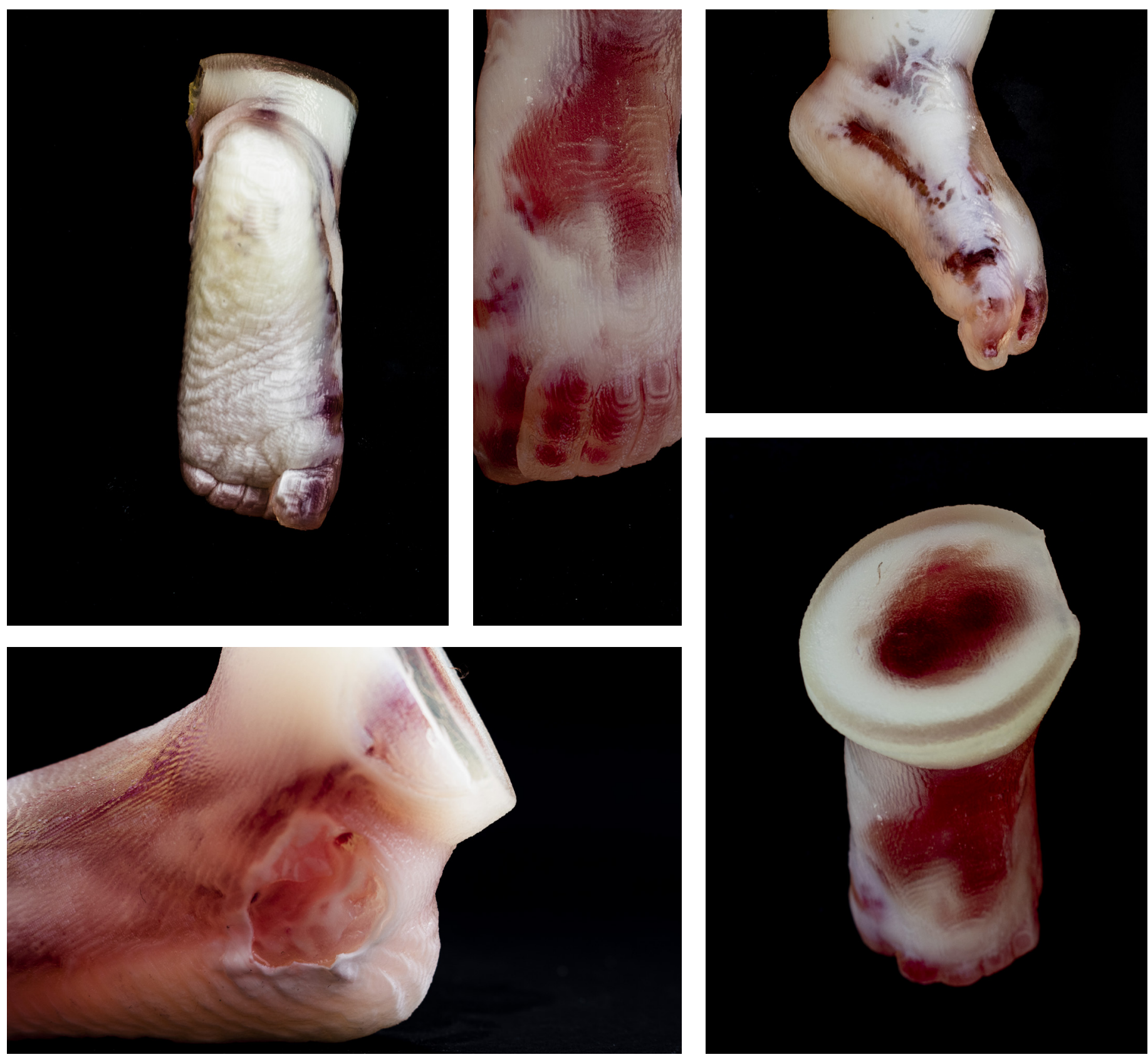

A i m

To test an area of anatomy with fat and skin, although this was unsuccessful in initial design experimentation. It was important to test this with a whole area of anatomy rather than with just a cross-section.
The 3D-print was successful. Applying a thicker layer of "skin" encased the SUP706 ${ }^{\mathrm{TM}}$ successfully, creating a realistic medical model that can be cut open, exposing the fat, ligaments, and bone. 

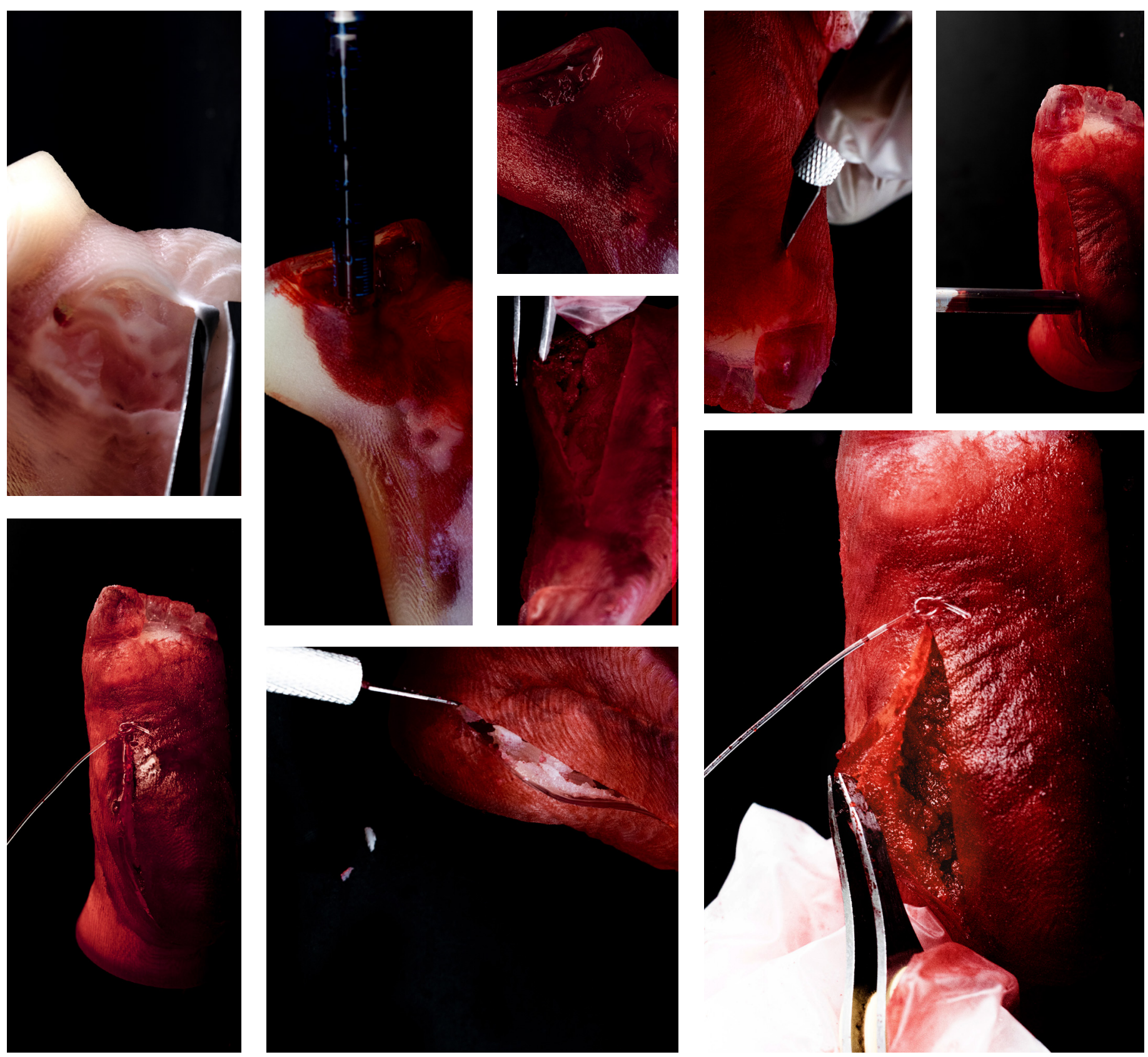

Figure 109. Application images of 3D-print \#36.

To show how models such as 3D-print \#36 can be contextualised and used in a medical setting. 


\section{2 .6}

\section{Fi n a I e s i g n}

Note: To create the 1:1 full scale 3D-print of a neonatal limb, the images were scaled into the 3D-printer's native $\mathrm{X}$ and $\mathrm{Y}$ resolutions and digital halftoning algorithms were used to create pixel combinations using the primary 3D-printer materials of VeroMagenta- $\mathrm{V}^{\mathrm{TM}}$ and Agilus $30^{\mathrm{TM}}$ with a $30 \%$ pixel diffusion ratio.

Left:

Figure 110. Digitally halftoned image of bitmap-based 3D-printed 1:1 scale neonatal leg (1/2,282 images processed for 3D-printing). 
\#37/ Neonatal Leg

Figure 111-120. Images of the final 1:1 model of the neonatal leg. The dimensions for the 1:1 neonatal leg were $\mathrm{X}=120.608 \mathrm{~mm}, \mathrm{Y}=$ $244.941 \mathrm{~mm}$, and $\mathrm{Z}=61.587 \mathrm{~mm}$.

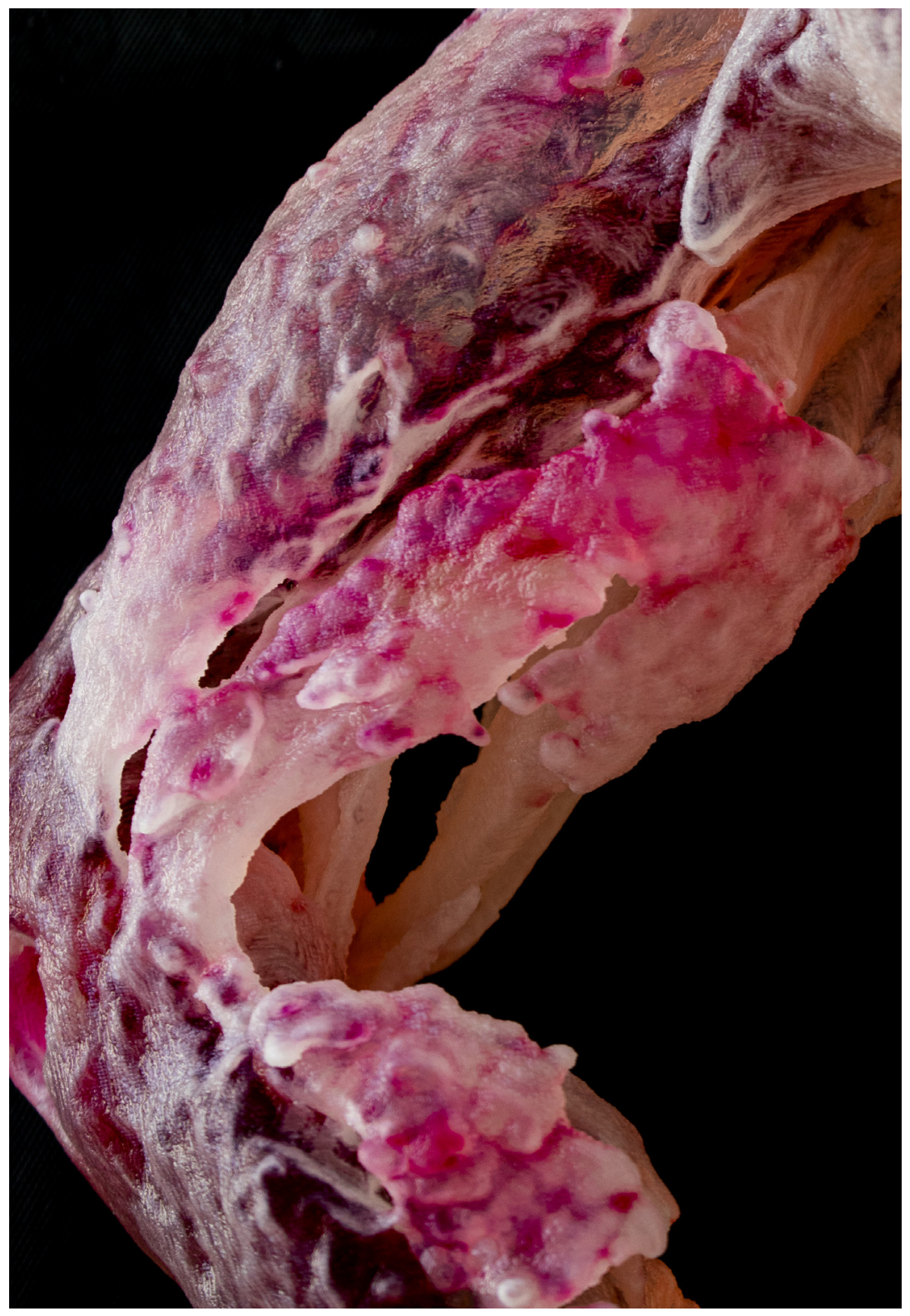

Note. Back of knee. 


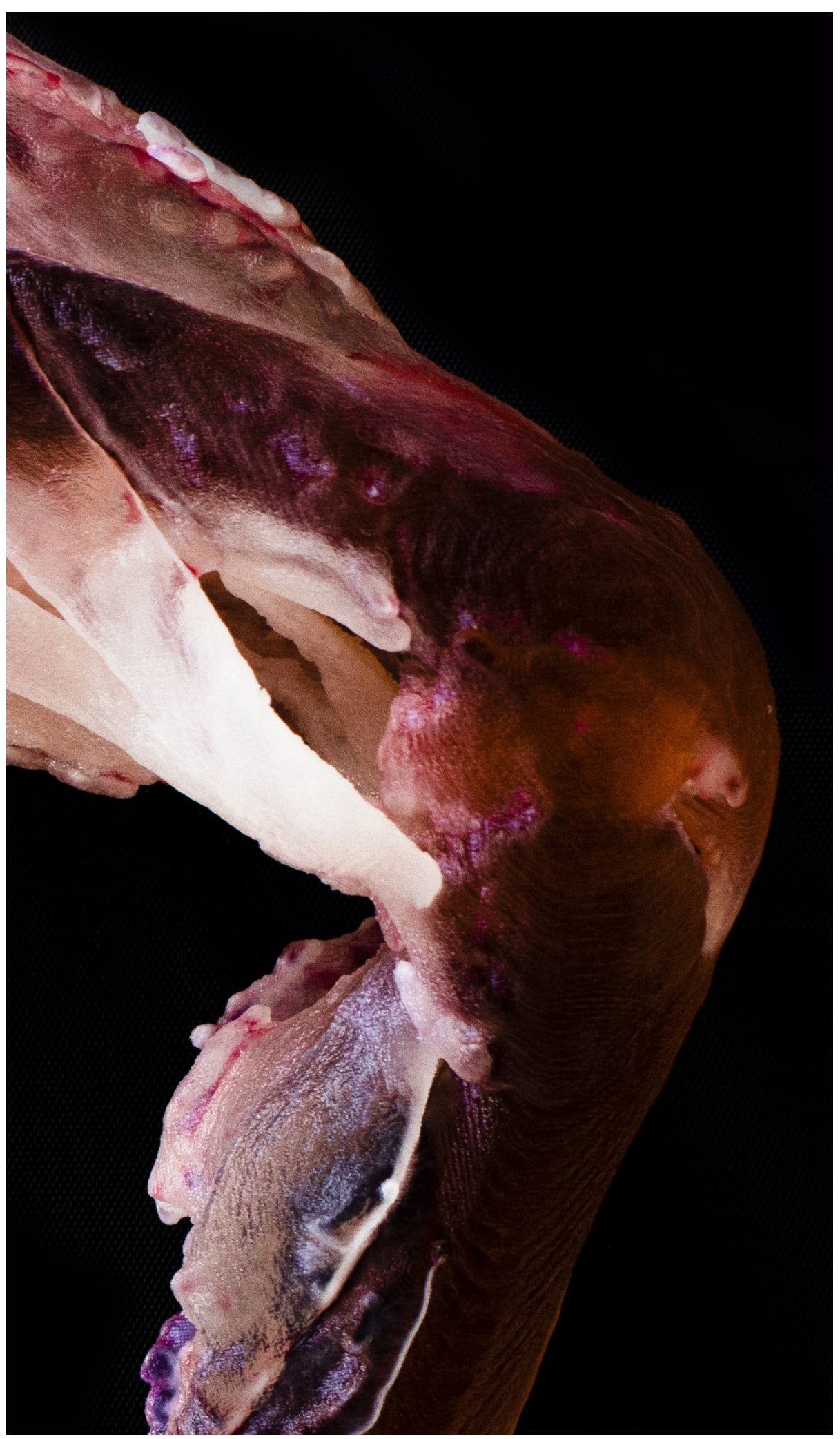

Note. Medial side of leg. 


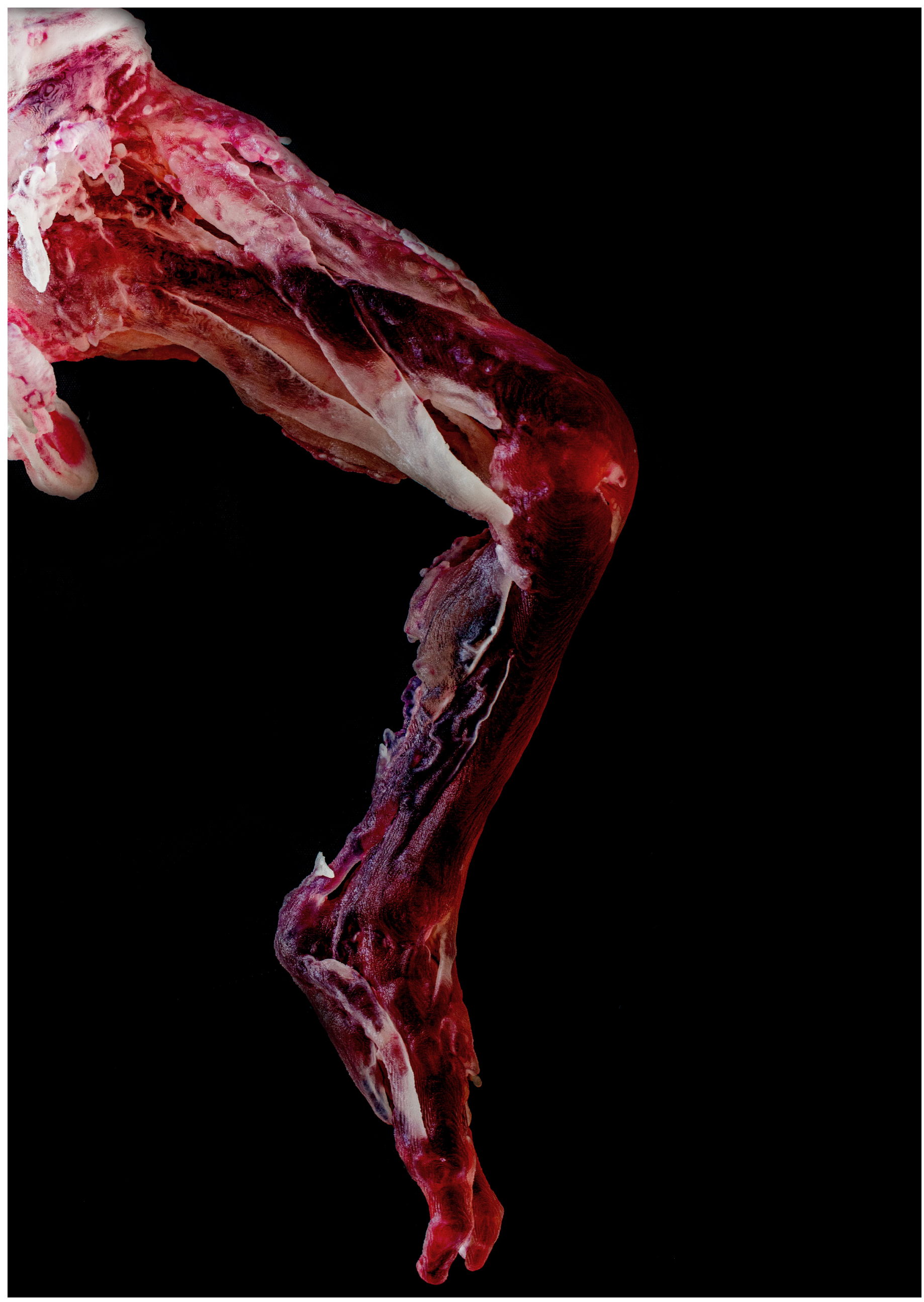




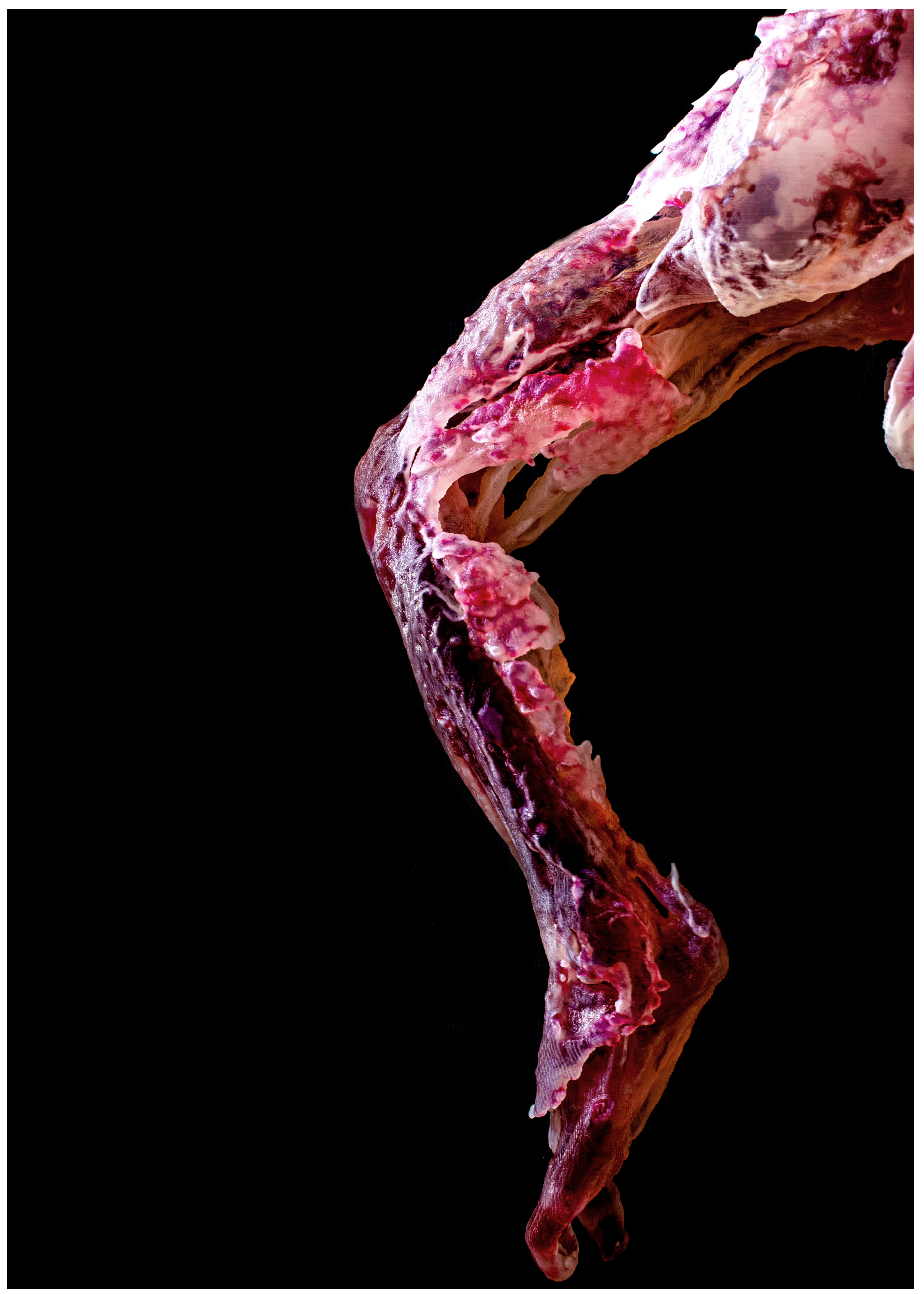




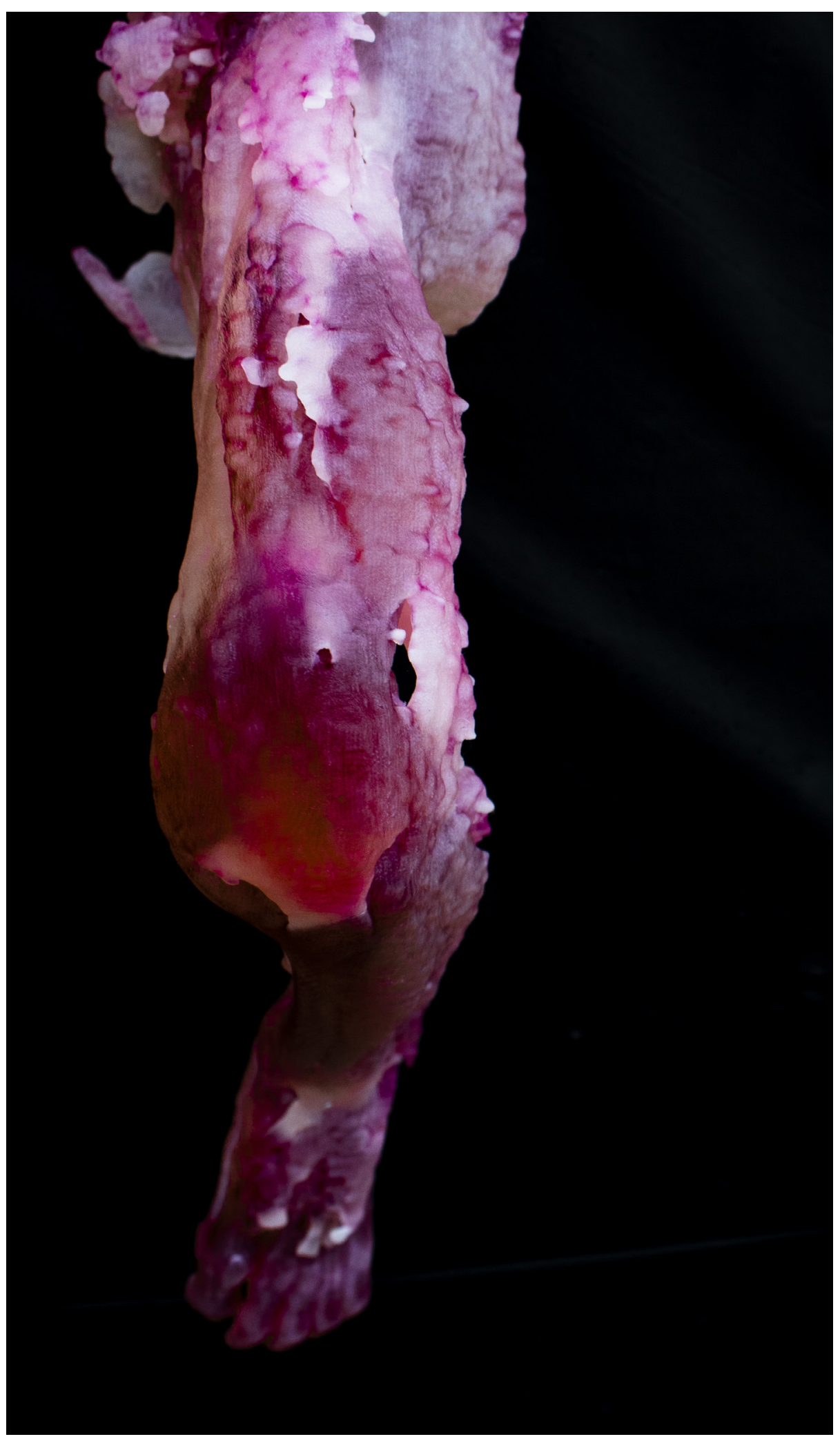

Note. Bird's eye view focussing on the knee. 


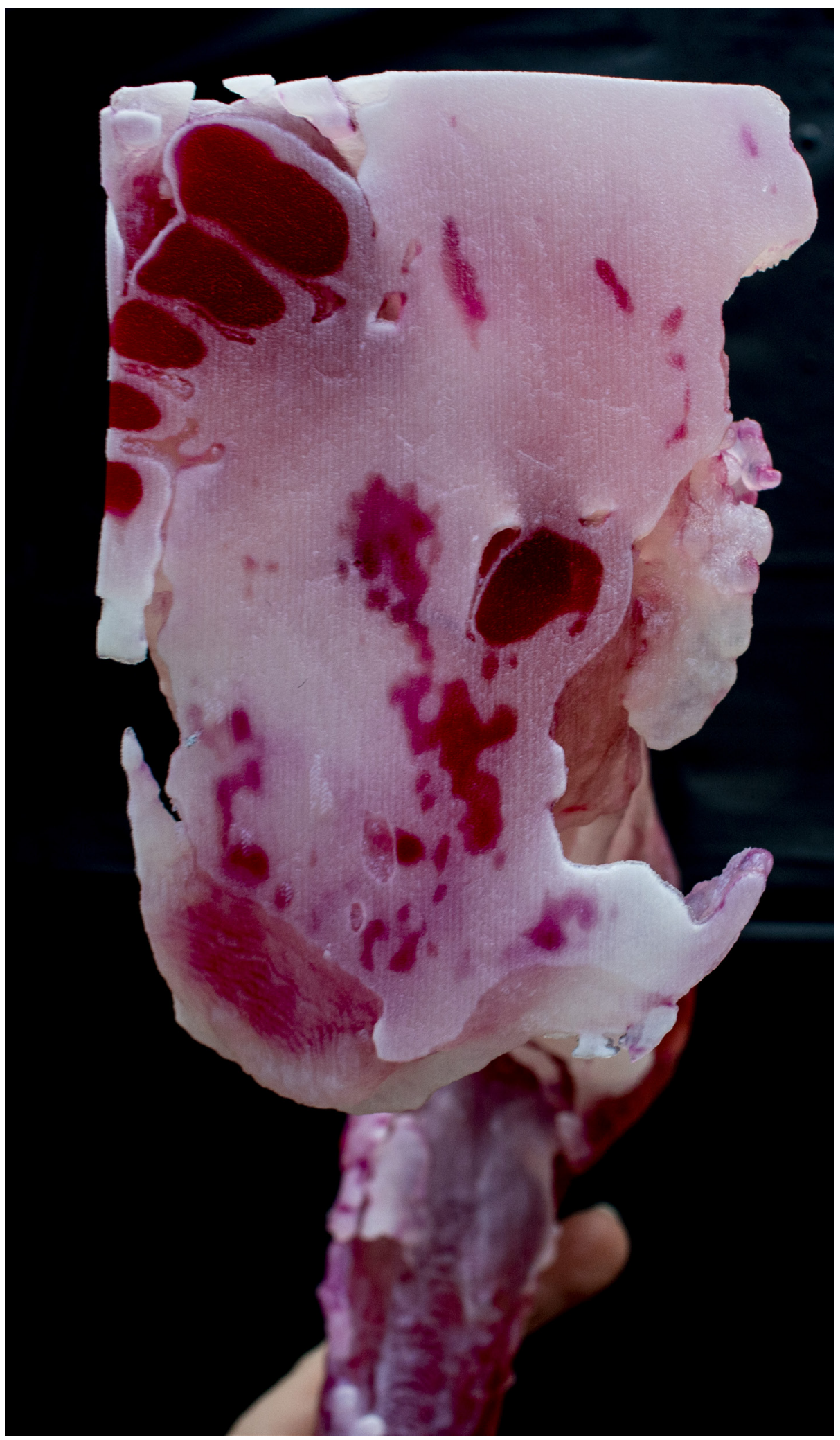

Note. Body midline (including a section of the spine) 


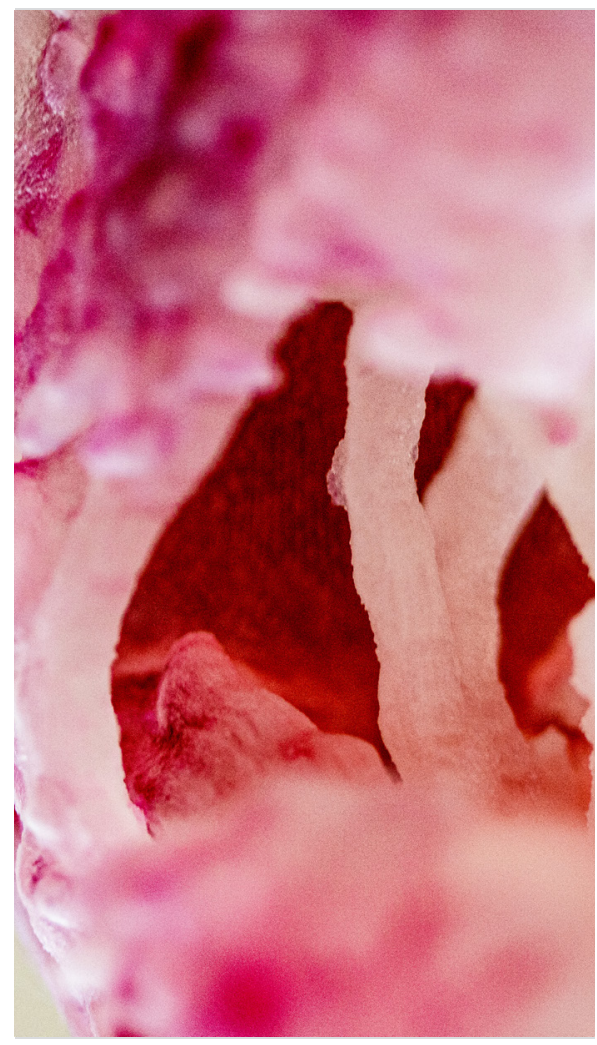

A

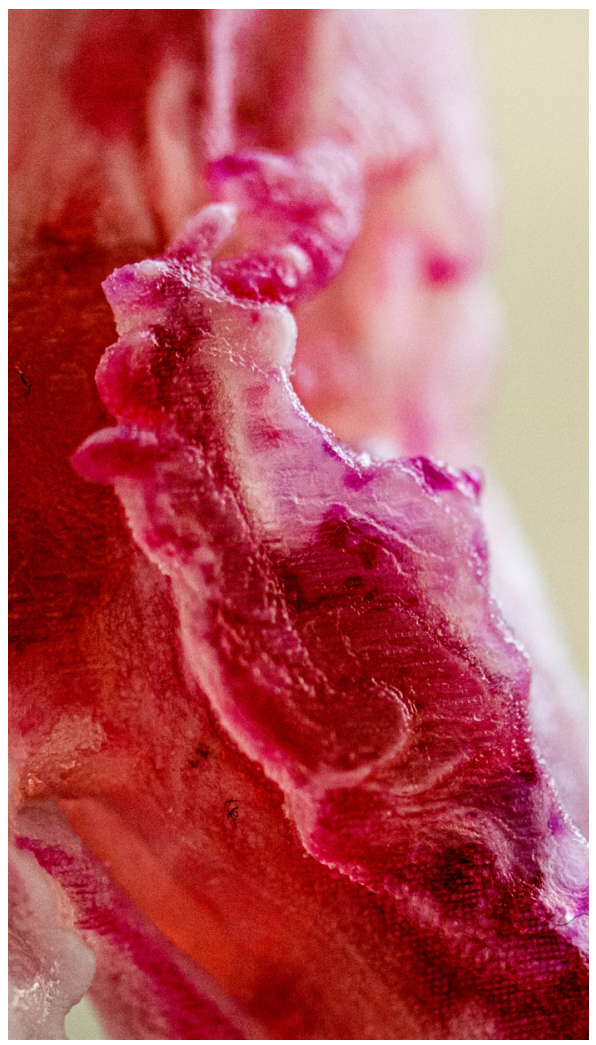

D
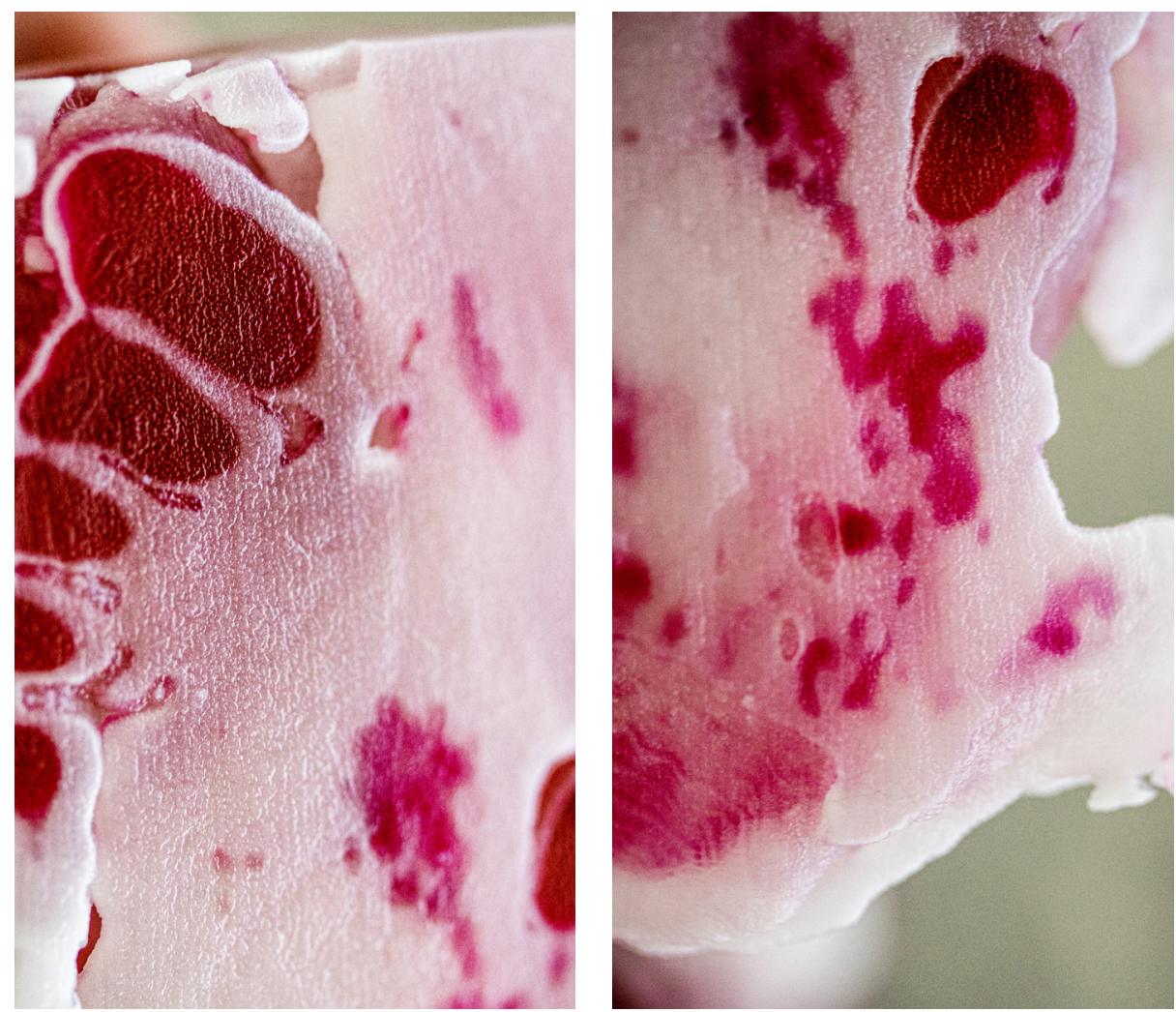

B

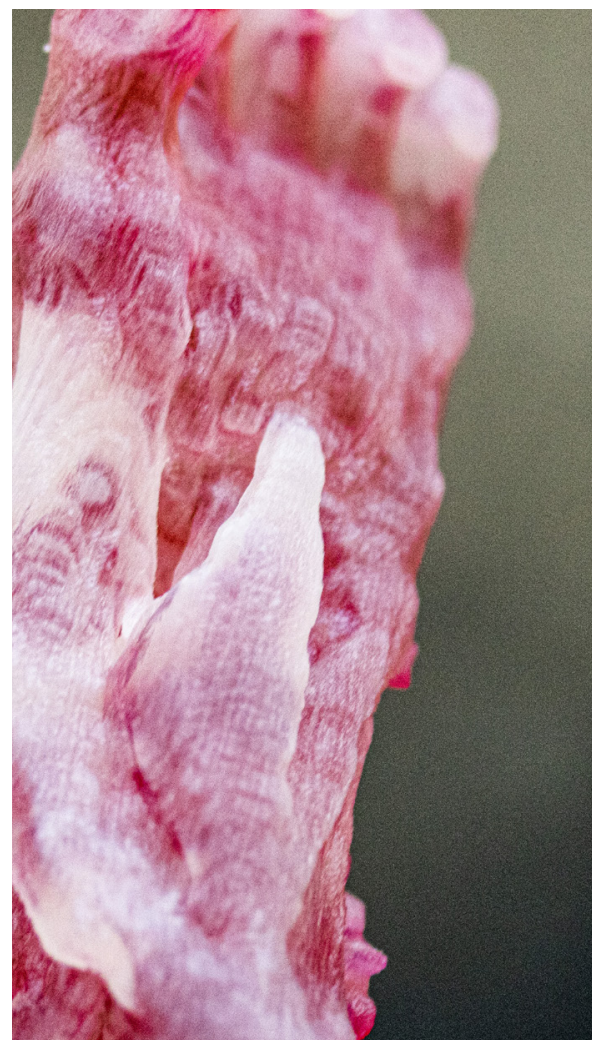

E
C

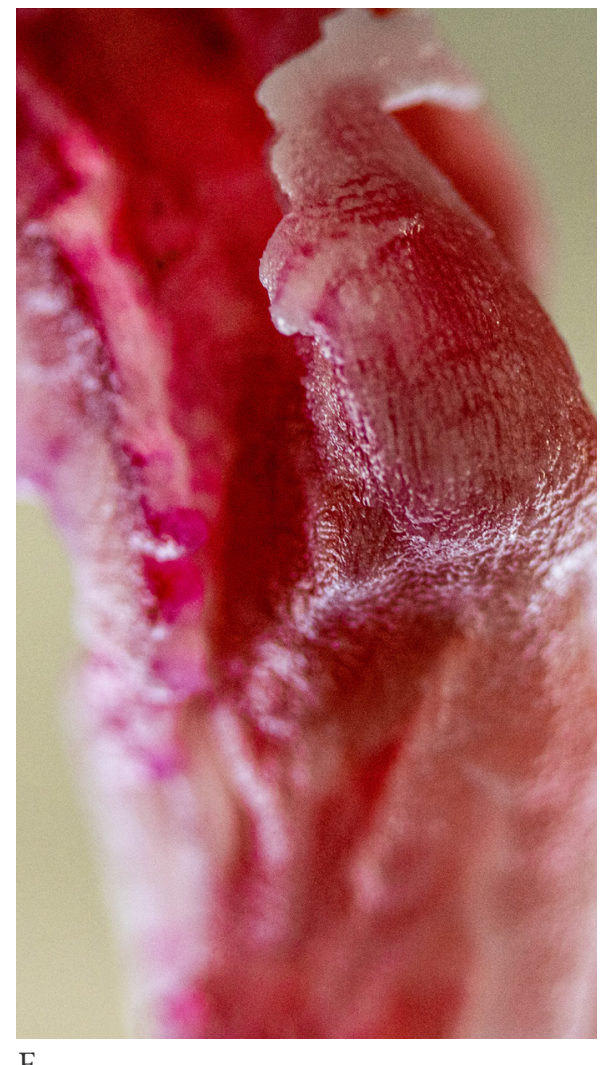

F

Figure 117. Close-up images of 1:1 bitmap-based 3D-printed neonatal leg. (A) Ligaments within the knee. (B) Close-up of the spine. (C) Texture and gradients down the body midline. (D) Close-up of side of the foot. (E) Bottom of foot. (F) Heel of foot. 


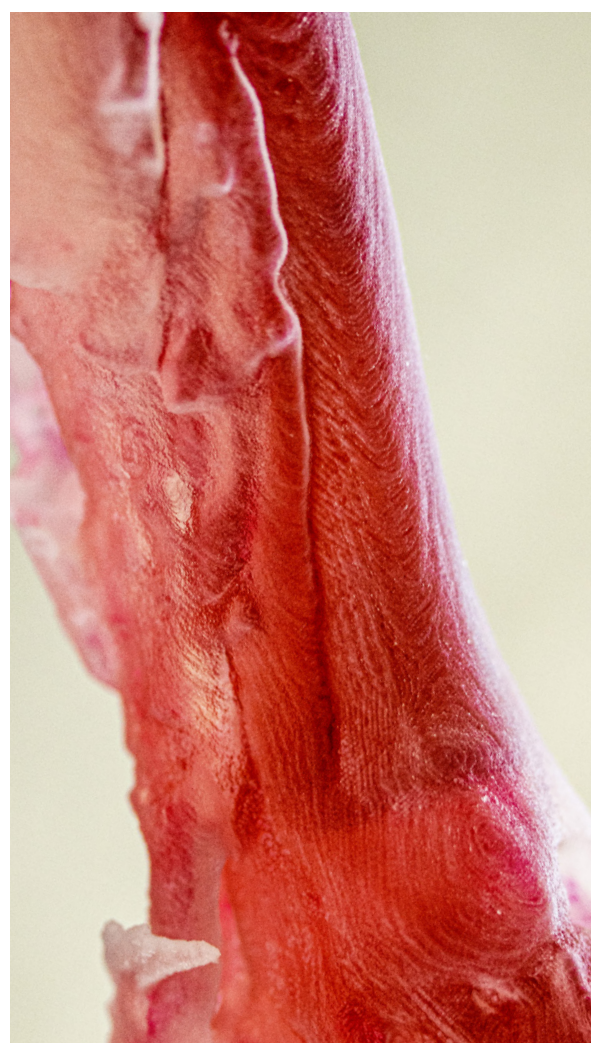

A

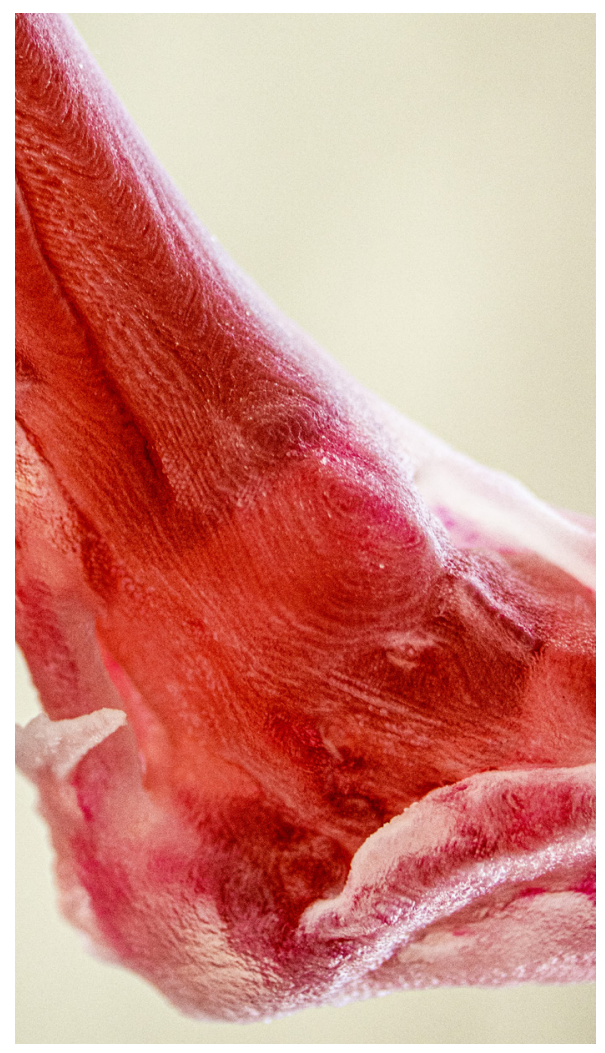

D

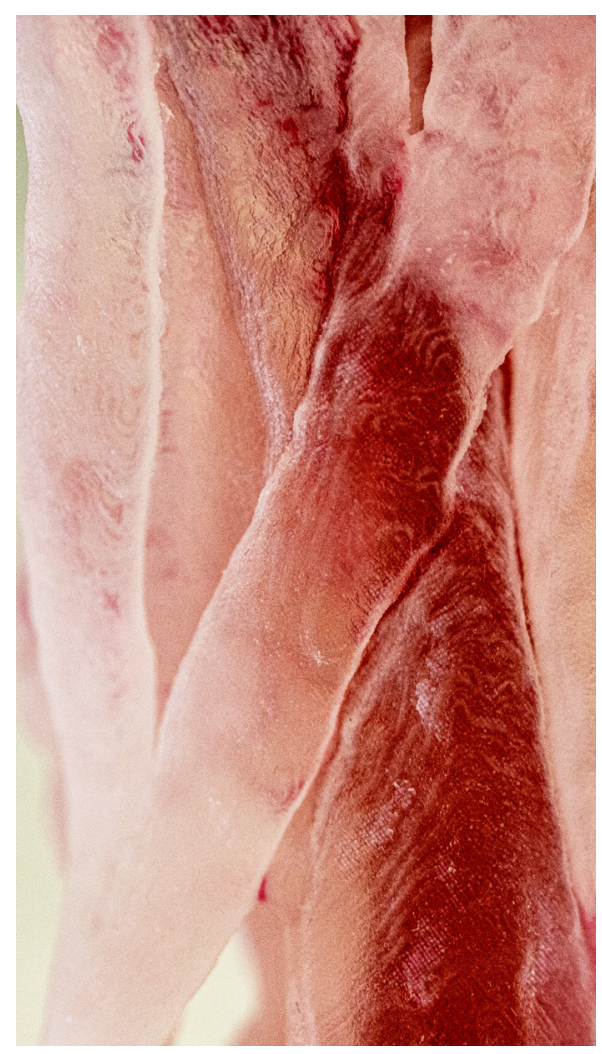

B

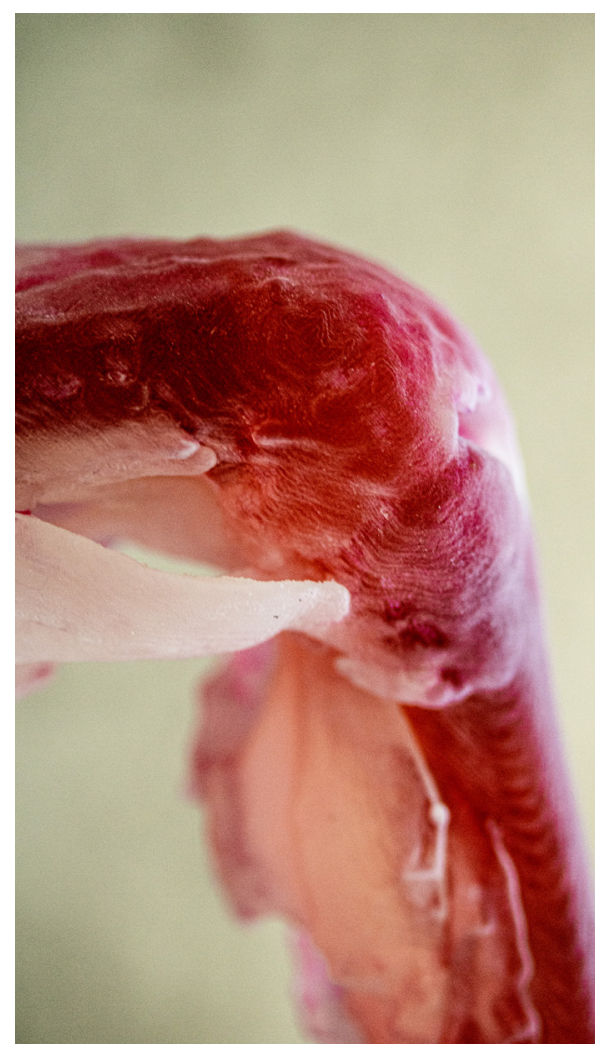

E

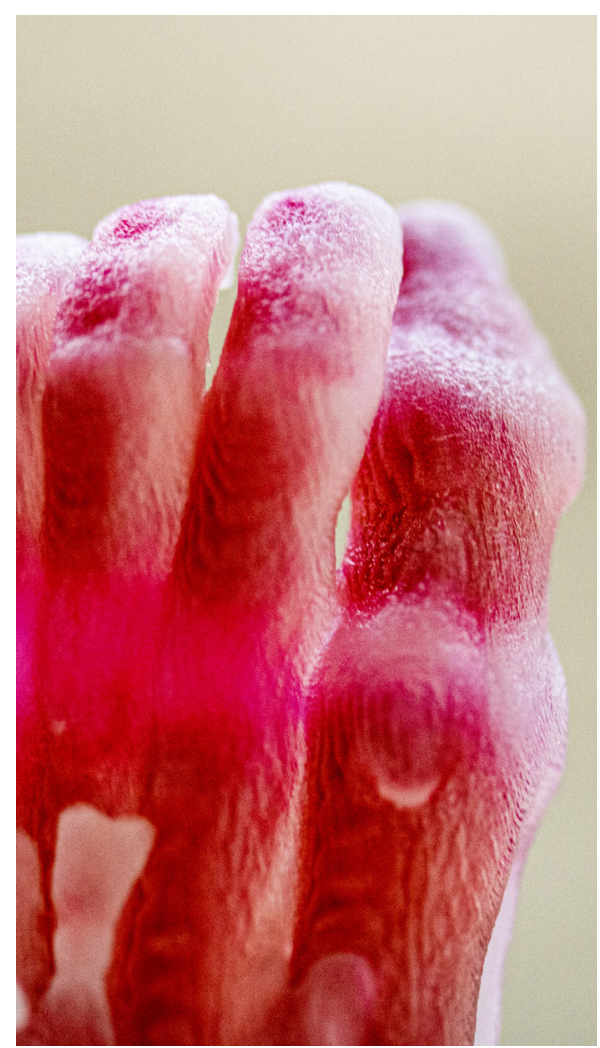

C

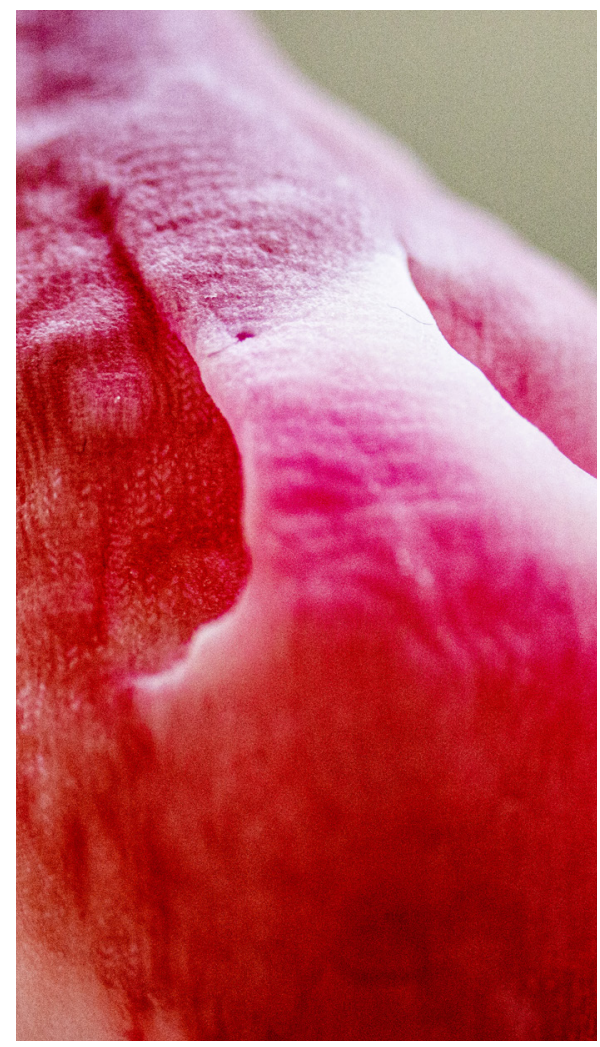

F

Figure 118. Close-up images of 1:1 bitmap-based 3D-printed neonatal leg. (A) Lateral side of ankle. (B) Close-up of crossing over muscles in the thigh. (C) Close-up of toes and the transition between Vero ${ }^{\mathrm{TM}}$ and Agilus30 $30^{\mathrm{TM}}$. (D) Lateral side of heel. (E) Medial side of knee. (F) Close-up of knee (patella). 

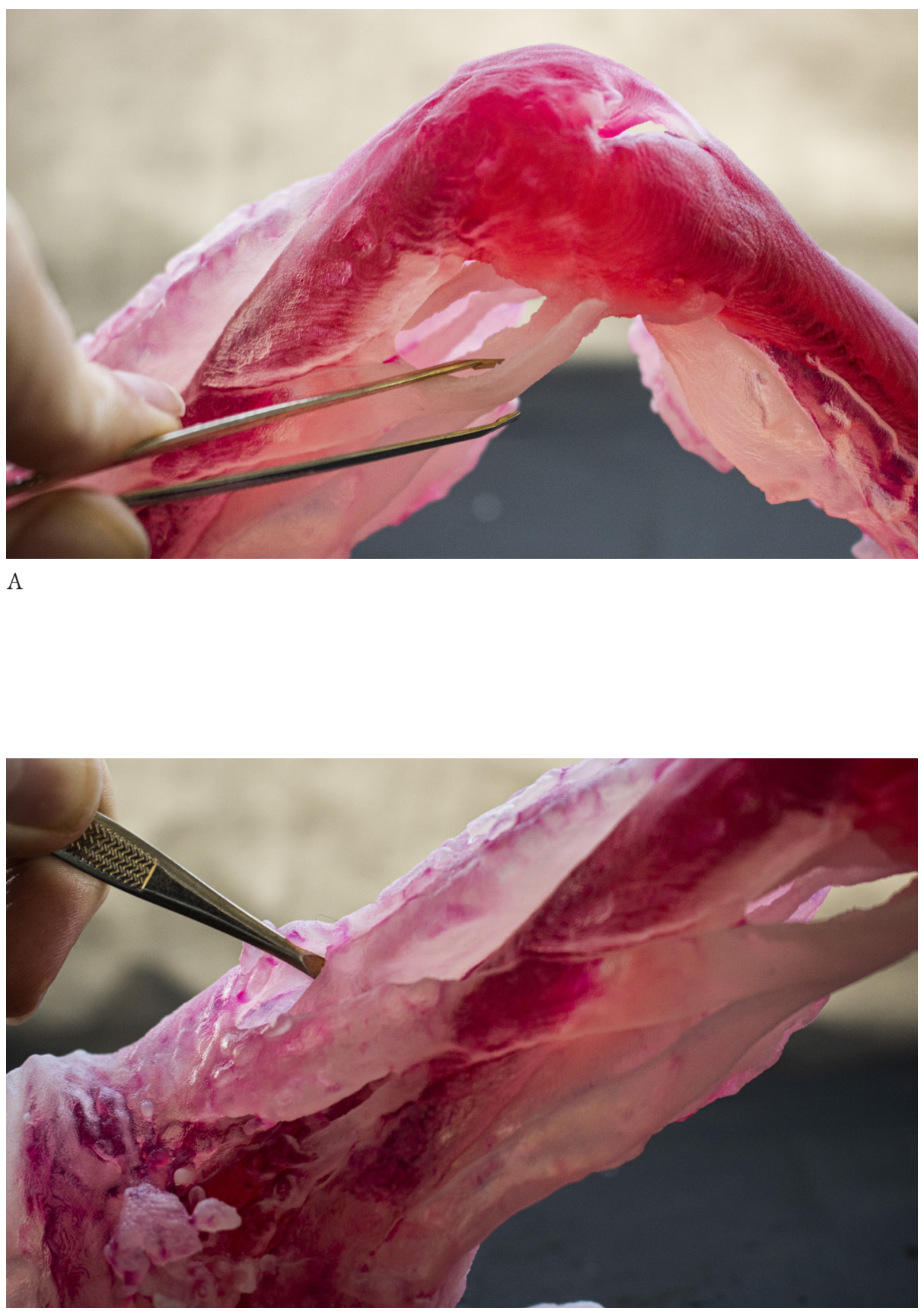

B

Figure 119. (A-D) Moving bitmap-based neonatal ligaments with tweezers. 


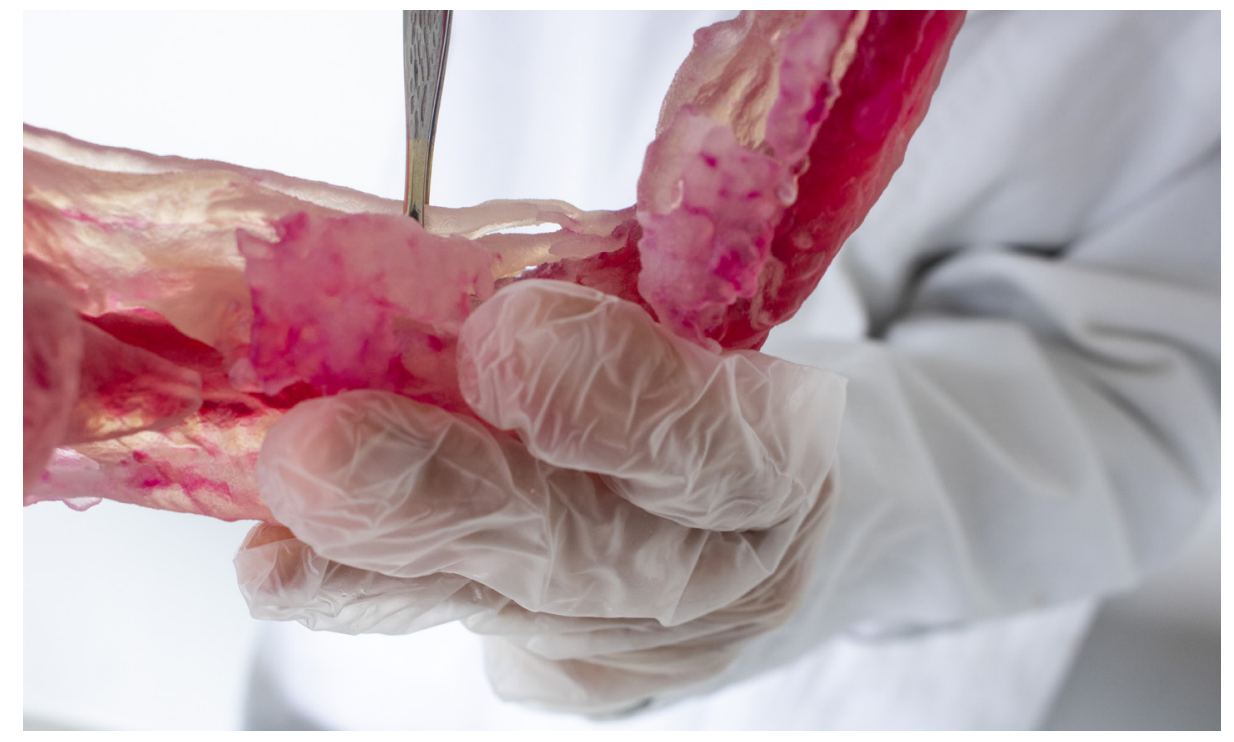

C

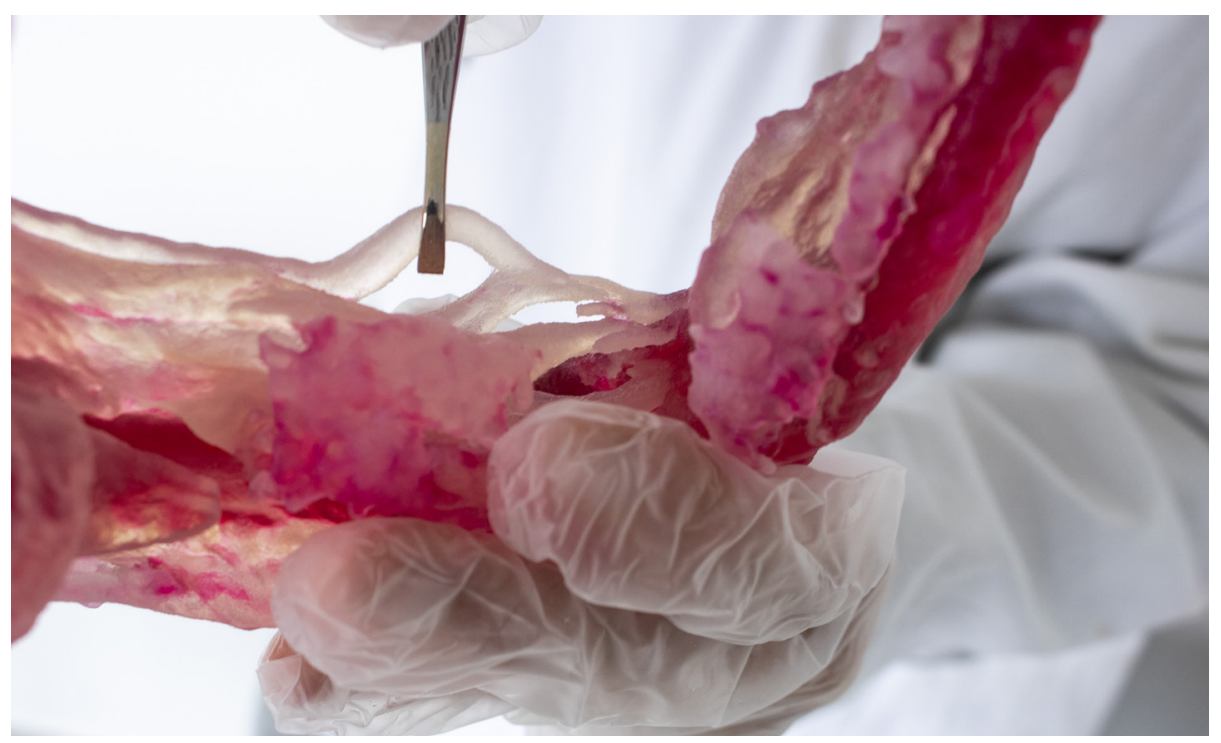

D 

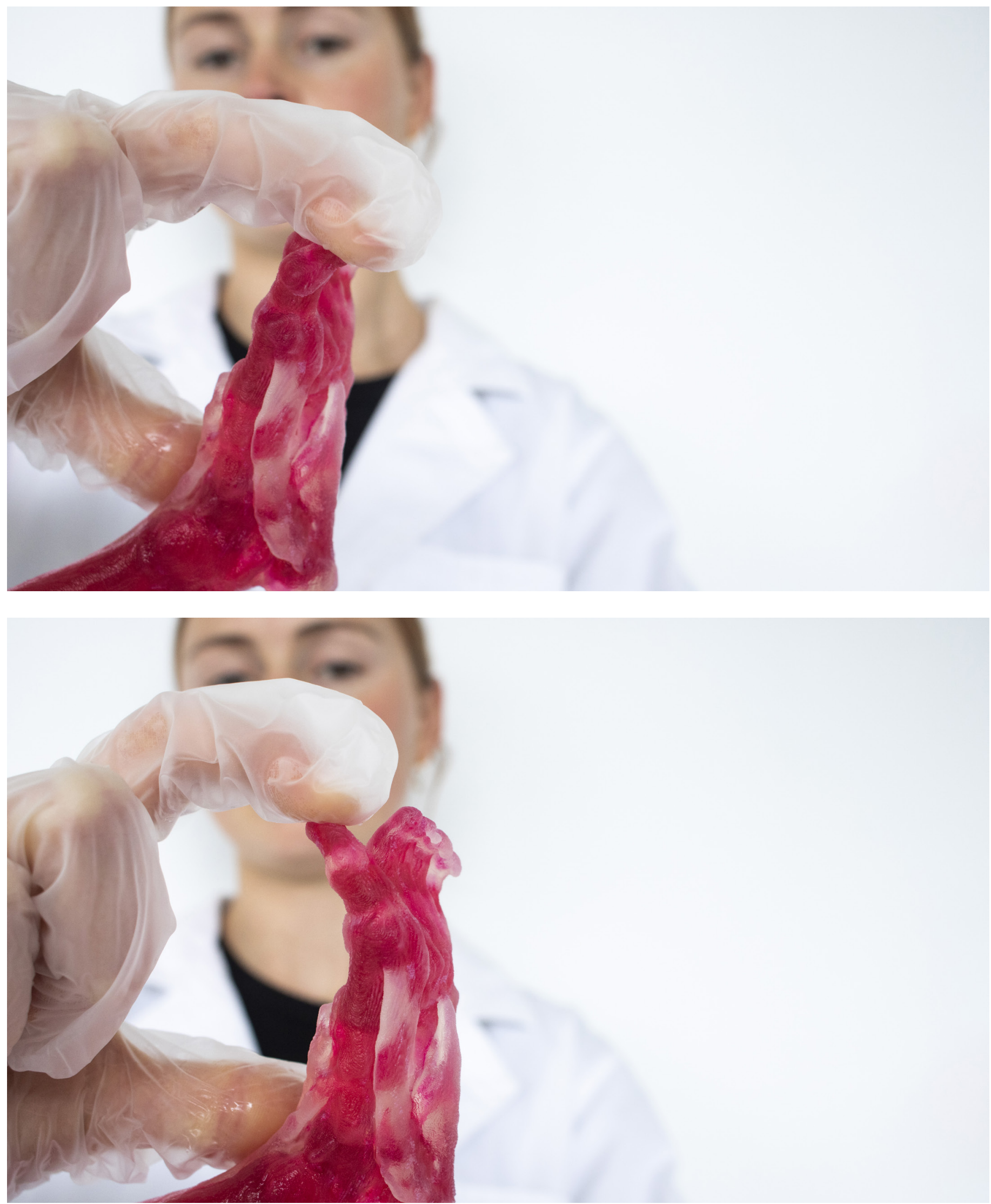

Figure 120. Articulating the bitmap-based neonatal toes demonstrating biomechanics. 

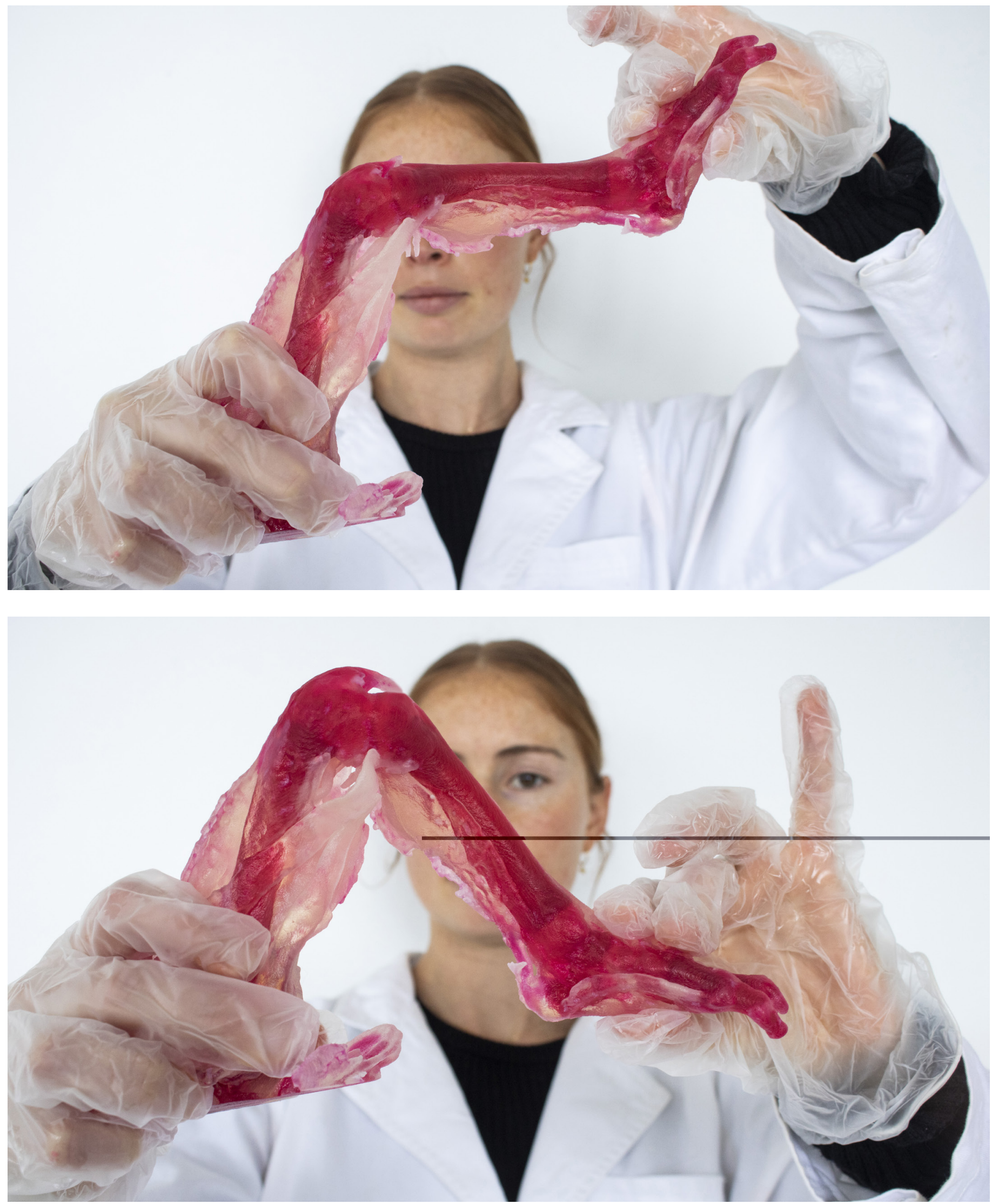

Figure 121. Articulating the bitmap-based neonatal knee joint demonstrating biomechanics. 


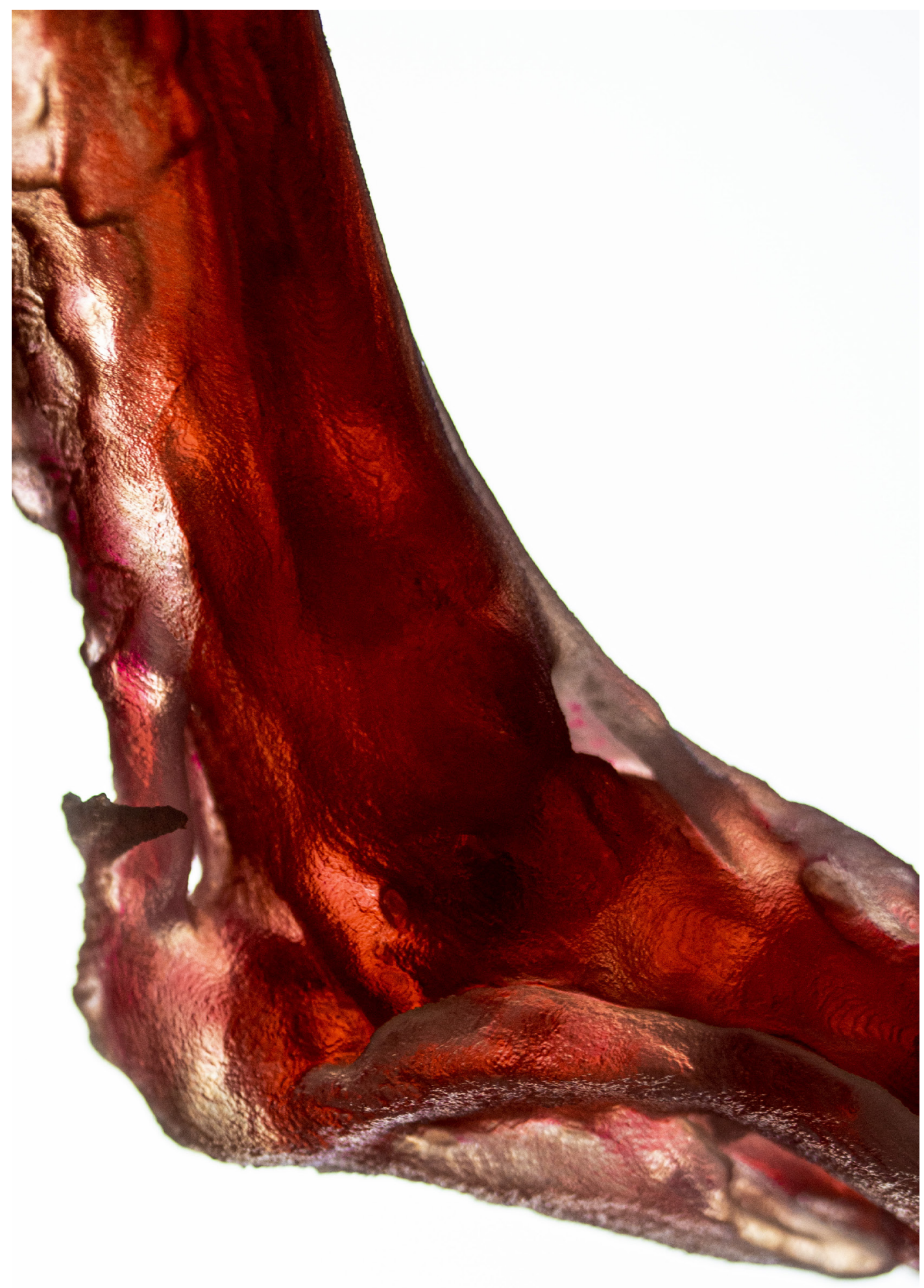

Figure 122. Close-up of bitmap-based neonatal leg (foot). 
4.2.7

Evaluation and Reflection

Case Study 2 helped to develop an effective bitmap-based 3D-printing workflow for the conversion of CT data into dynamic 3D-printed models. This workflow was able to translate the biomedical imaging data into 3D-printed models with gradients in density, ranging from very soft 3D-printed material (mimicking fat) to harder areas of anatomy such as bone. Evaluation of the bitmap-based 3D-Prints conducted in Case Study 2 was conducted through material property observation, tactile analysis, and photographic documentation. The 3D-prints were also presented via Video Call to an Expert Reviewer, Dr. Ali Mirijilili, who gave feedback virtually and in person at a MedTech Core meeting.

MOVING FORWARD

Based on the results of Case Study 2, an interesting avenue of exploration for future research would be to look into creating more realistic models with materials that are softer - mimicking the qualities of human anatomy to a higher degree of realism. 

conducted in this Case Study was done

through material property observation,

tactile analysis and photographic documentation. The 3D-Prints were also presented via Video Call to an Expert Reviewer, Dr. Ali Mirjalili, who gave feedback virtually.

Case Study 2 helped to develop an effective bitmap-based 3D-Printing workflow for the conversion of Biomedical Imaging data into dynamic 3D-printed models. This workflow was able to translate the Biomedical Imaging data into 3D-printed models with gradients in density, ranging from very soft 3D-printed material (mimicking fat) to harder areas of anatomy such as bone.

Based on tactile and visual analysis of the 3D-prints and feedback from expert opinions, it is clear that this workflow is relatively unprecedented as the use of soft (Agilus30 $30^{\mathrm{TM}^{\mathrm{M}}}$ ) and hard (Vero ${ }^{\mathrm{TM}}$ ) materials blended within bitmap-based 3D-prints. The method is novel and limited studies exist using this technique.

Case Study 2 highlighted the potential of bitmap-based 3D-printing using varying densities for the purpose of biomechanical research, medical education, preoperative planning and simulation.

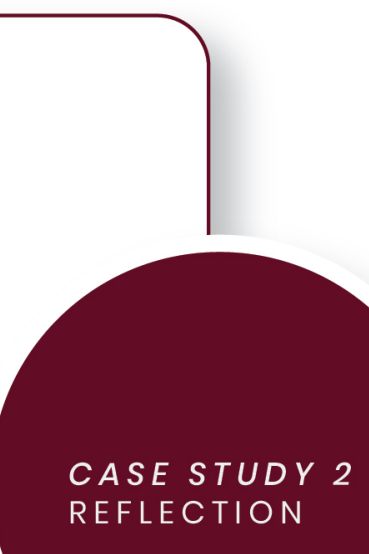

THINGS TO CONSIDER

It is important to consider that there is no research conducted that quantified whether Stratasys J750 $0^{\mathrm{TM}}$ 3D-printing materials are equivalent to anatomy. Evaluation of Case Study Two was qualitative and was based on whether a bitmap-based 3D-printing workflow was possible.

Although the softest material available to 3D-print with using the Stratasys J750 ${ }^{\mathrm{TM}}$ 3D-Printer is Agilus $30^{\mathrm{TM}}$, it is still not as soft as human tissue. The resulting 3D-prints moved in a similar way to human anatomy but still felt stiff in comparison. Also, Vero ${ }^{\mathrm{TM}}$, although hard like bone, maybe did not have the physical properties needed on bone. For example, for simulation purposes it is imperative that bone does not melt when drilled.

Dr. Ali Mirjalili commented that some very thorough traditional segmentation methods can produce highly accurate 3D-prints. Through a process of manual thresholding, you can achieve highly-detailed models even though the process can be quite time-consuming. Therefore, it will be important to compare:

a. Voxel-printing using a High-Quality CT scan

b. Voxel-printing using a Normal CT scan

c. STL segmentation mesh-based 3D-printing using a clinically segmented model.

Following 3D-printing these three different types of models, a comparison can be conducted between the three models and the advantages and disadvantages should be outlined. Suggestions can be made for where each method could be implemented depending on the use and quality of the model. 


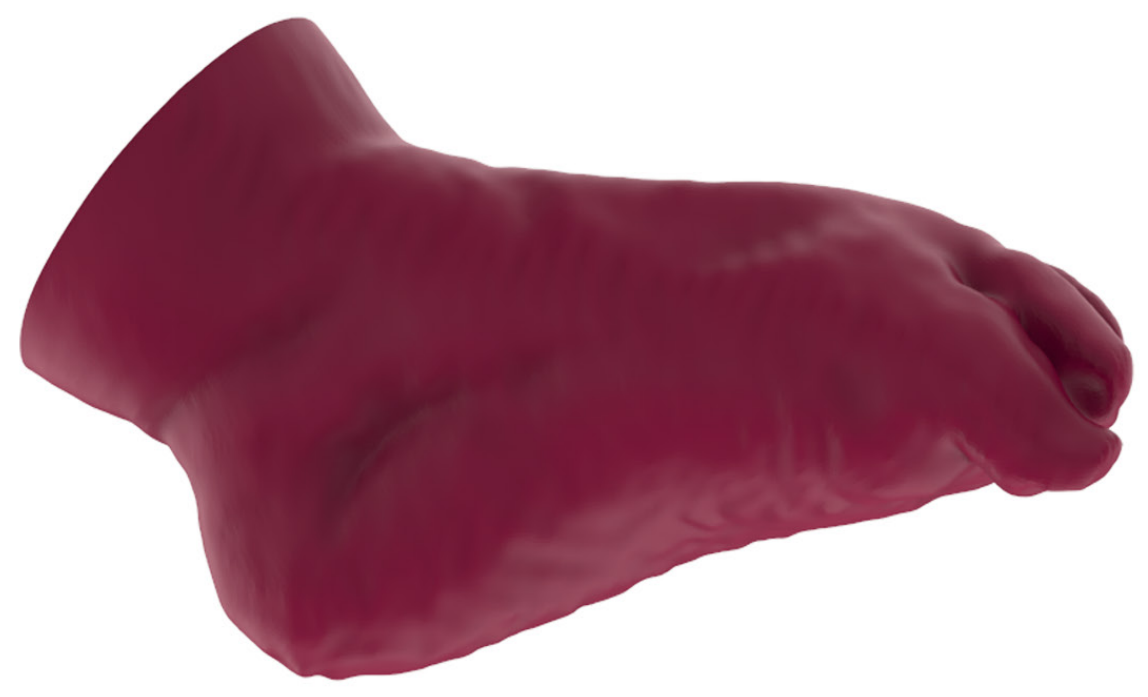

1
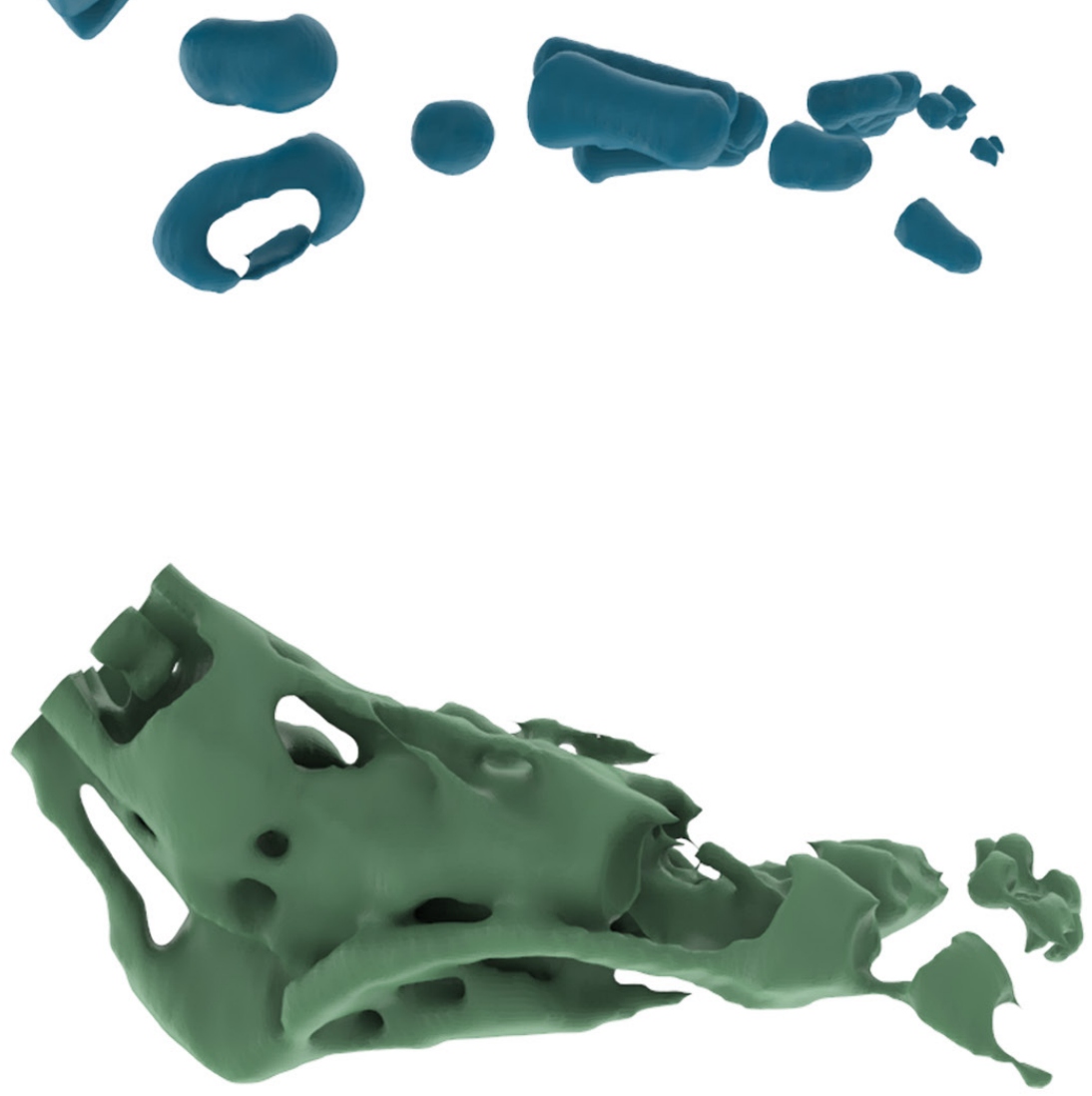
PRACTICAL ACTION RESEARCH

\subsection{A S E S T U Y 3}

Testing Novel Medical Modelling 3D-Printing Materials on the Stratasys J750 TM

Digital Anatomy ${ }^{\mathrm{TM}}$ 3D-Printer (DAP) released in November 2019.

Left.

Figure 124. Neonatal STL (mesh files) used to create the mesh-based neonatal feet on the Stratasys $\mathrm{J} 750^{\mathrm{TM}}$ Digital Anatomy ${ }^{\mathrm{TM}}$ 3D-Printer. 
4. 3.1

overview

Case Study 3 focussed on developing swatches and test 3D-prints of anatomy to test on the brand new Stratasys J750 ${ }^{\mathrm{TM}}$ Digital Anatomy ${ }^{\mathrm{TM}}$ 3D-Printer based in Shanghai, China. The aim of Case Study 3 was to 3D-print swatches of new (more developed) 3D-printing materials designed for medical modelling. In 2019, Stratasys released a new 3D-Printer and 3D-printing materials that aim to combat the rigidity of polyjet materials - a challenge that arose in Case Study 2. The key new base materials are TissueMatrix ${ }^{\mathrm{TM}}$ (the softest translucent material commercially available), BoneMatrix ${ }^{\mathrm{TM}}$ (a dense, yet flexible, material) and GelMatrix $^{\mathrm{TM}}$ (a support material that can be easily removed) (Stratasys J750 ${ }^{\mathrm{TM}}$ Digital Anatomy ${ }^{\text {TM }}$ 3D-Printer, 2019). A combination (mixing ratios) of the six materials available for 3D-printing at one time on the Stratasys $\mathrm{J} 750^{\mathrm{TM}}$ Digital Anatomy ${ }^{\mathrm{TM}}$ 3D-Printer can produce a range of 'Digital Anatomy' materials such as the 'Myocardium' or 'Long Bone'. These materials used in combination have the potential to provide an unprecedented level of realism in medical models (Severseike et al., 2019).

3D-printed models were produced using a traditional mesh-based 3D-printing workflow. Generating meshes on CAD software meant that three-dimensional forms could be additively manufactured from computed generated geometries instead of bitmap images. Material swatches were produced using Austodesk Fusion 360 and were assigned with the Digital Anatomy ${ }^{\mathrm{TM}}$ materials using GradCAD Print. The medical models of the neonatal foot were developed on medical imaging software using traditional thresholding, segmentation and computer processing methods. Currently, the available version of GrabCAD for the Stratasys $\mathrm{J}^{750^{\mathrm{TM}}}$ Digital Anatomy ${ }^{\mathrm{TM}}$ 3D-Printer does not have a GrabCAD Voxel Print Utility add-on.

Parallel prototyping was used to produce a large batch of 3D-prints overseas. 
4.3.2

Criteria for case study 3

Case Study 3 focussed on producing swatches of novel 3D-printing materials by creating a series of design experiments and swatches. The focus of Case Study 3 was on testing the materials - reflecting on how they moved and felt in comparison to the Stratasys J750 TM 3D-printing materials. CT imaging data in this Case Study 3 was the same as Case Study 2.

C RITERIA

1. Explore the Stratasys $\mathrm{J}^{750^{\mathrm{TM}}}$ Digital Anatomy $^{\mathrm{TM}}$ 3D-Printer materials. Using CAD software, a series of swatches should be developed to test every Digital Anatomy ${ }^{\mathrm{TM}}$ material available for 3D-printing on GrabCAD Print.

2. Using traditional mesh-based segmentation techniques, use the Stratasys $\mathrm{J}^{750^{\mathrm{TM}}}{ }^{\mathrm{Digital}}$ Anatomy ${ }^{\mathrm{TM}}$ 3D-Printer to create medical models that mimic anatomy to a high-fidelity.

Traditional segmentation techniques should develop sections of a paediatric foot using the same dataset as Case Study 2. The three anatomical focus areas are the bone, ligaments and soft tissues and fat. These segmentations should preserve the most amount of original data possible.

3. Use the information from both Case Studies 1, 2 and 3 outline the potential of bitmap-based 3D-printing (Voxel Print Utility) using the Stratasys $\mathrm{J}^{750^{\text {TM }}}{ }^{\text {Digital Anatomy }}{ }^{\text {TM }}$ 3D-printer materials.

By comparing the mesh-based model and bitmap-based model of the paediatric foot as well as materials using both machines will set up the groundwork for future studies using the Stratasys J750 ${ }^{\mathrm{TM}}$ Digital Anatomy ${ }^{\mathrm{TM}}$ 3D-Printer. Speculating how the Voxel Print Utility add-on could function on the Stratasys J750 ${ }^{\mathrm{TM}}$ Digital Anatomy ${ }^{\mathrm{TM}}$ 3D-Printer may provoke thought about the potential of bitmapbased medical modelling. 


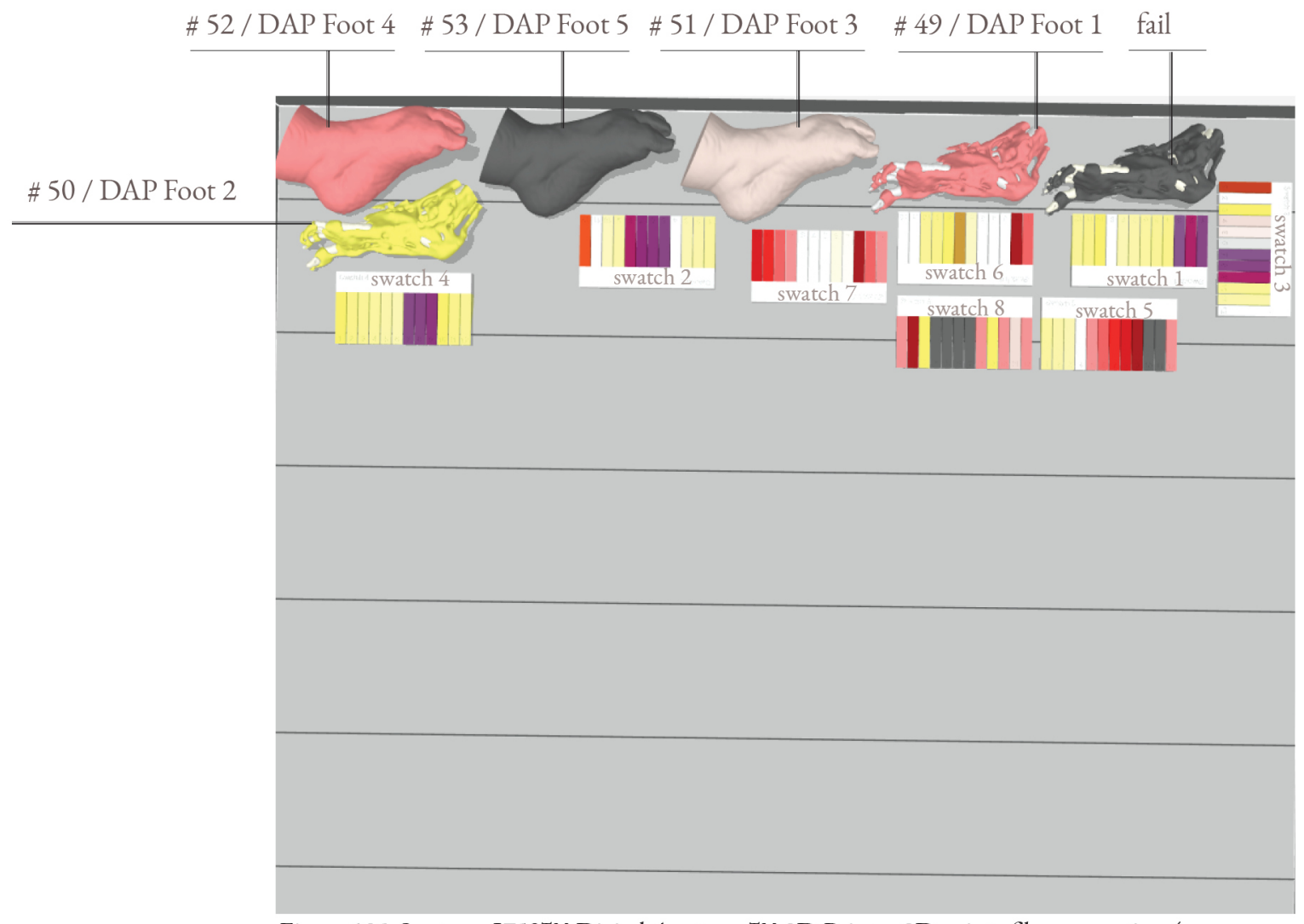

Figure 125. Stratasys J750 TM Digital Anatomy ${ }^{\mathrm{TM}}$ 3D-Printer 3D-prints file-preparation (appearance on GrabCAD Print software with assigned materials.

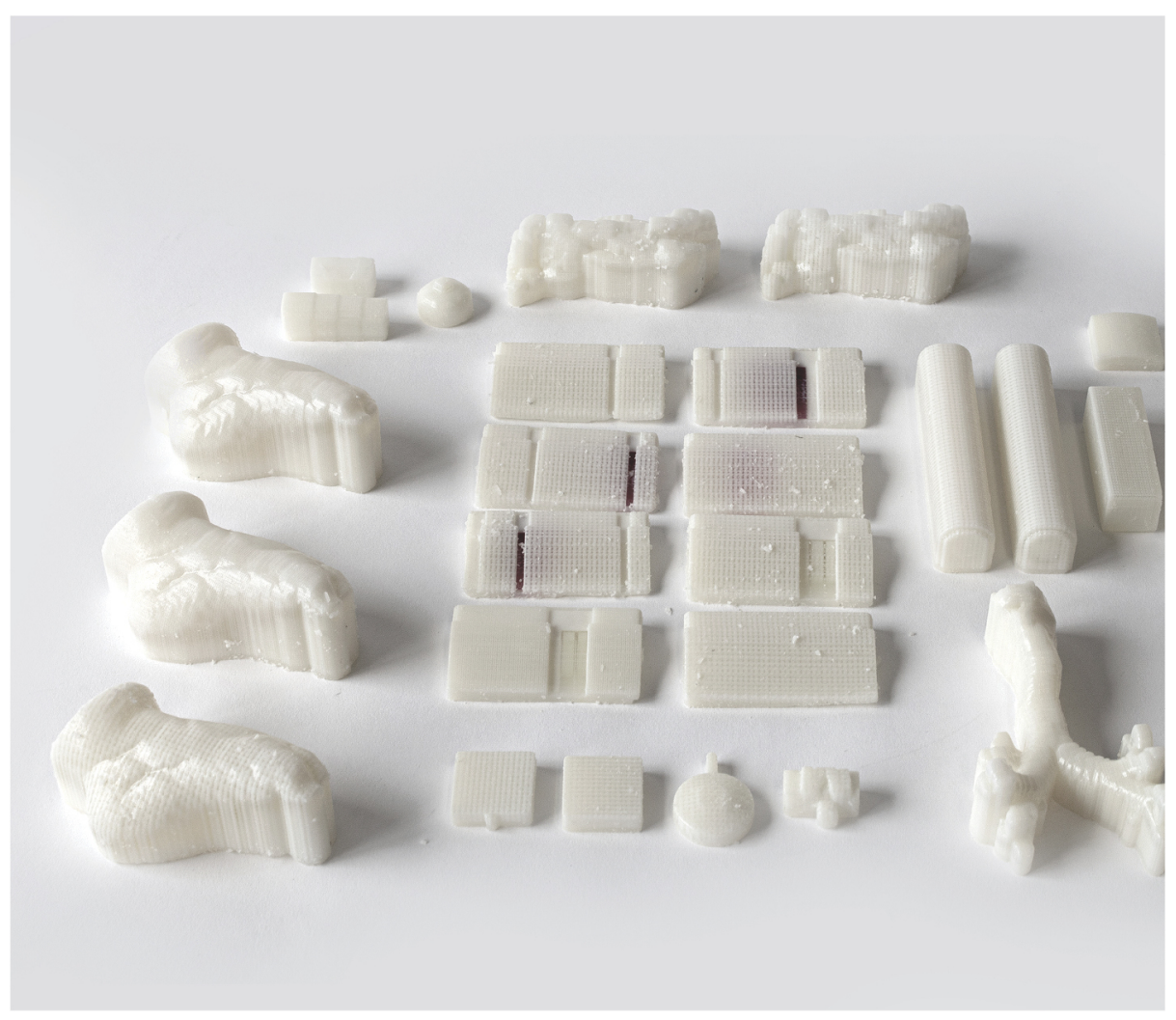

Figure 126. Stratasys J750 ${ }^{\mathrm{TM}}$ Digital Anatomy ${ }^{\mathrm{TM}} 3 \mathrm{D}-\mathrm{P}$ rinter 3D-prints coated in SUP705 ${ }^{\mathrm{TM}}$. 

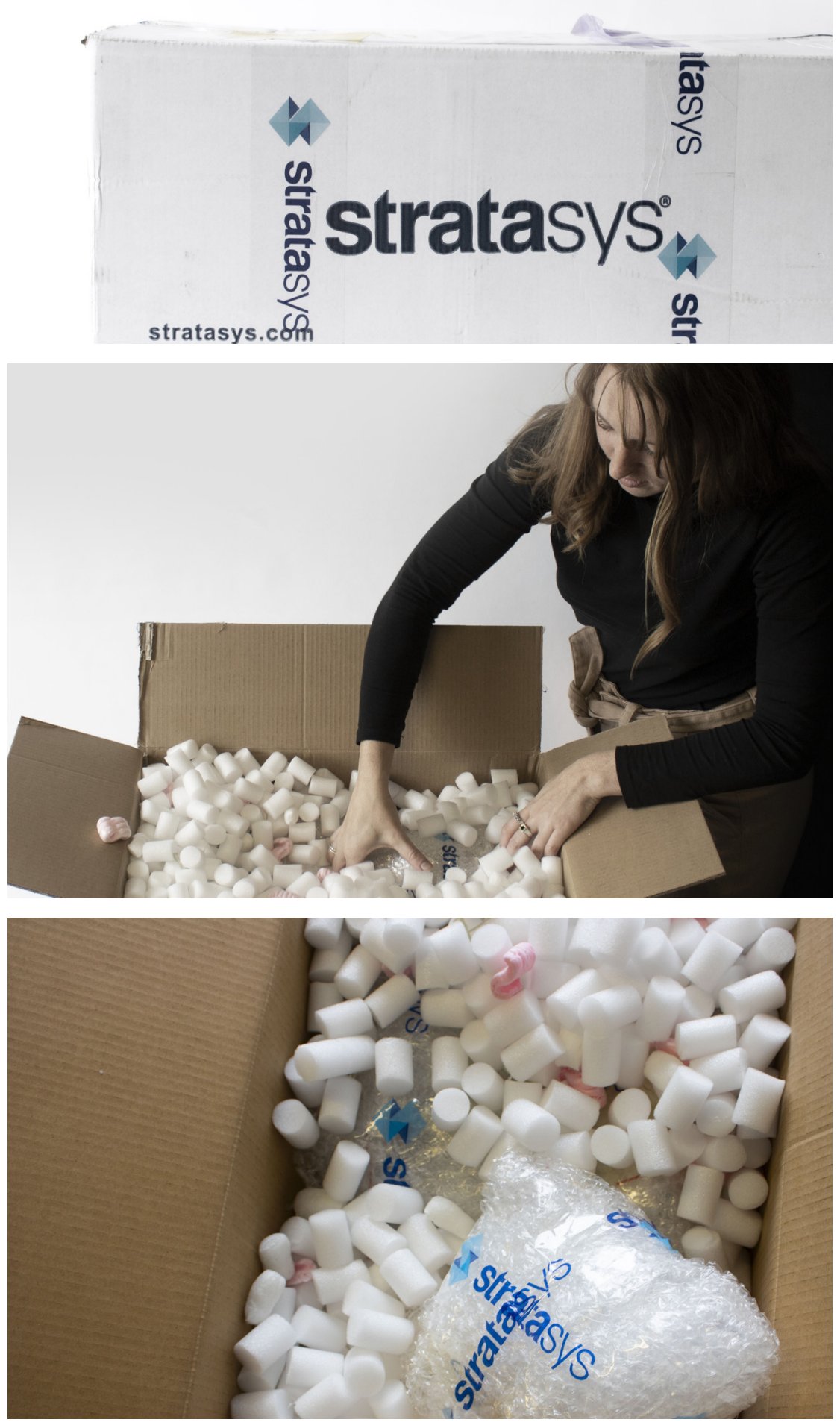

Figure 127. Receiving and opening Stratasys J750 ${ }^{\mathrm{TM}}$ Digital Anatomy ${ }^{\mathrm{TM}}$ 3D-Printer 3D-prints from Stratasys in Shanghai, China. 


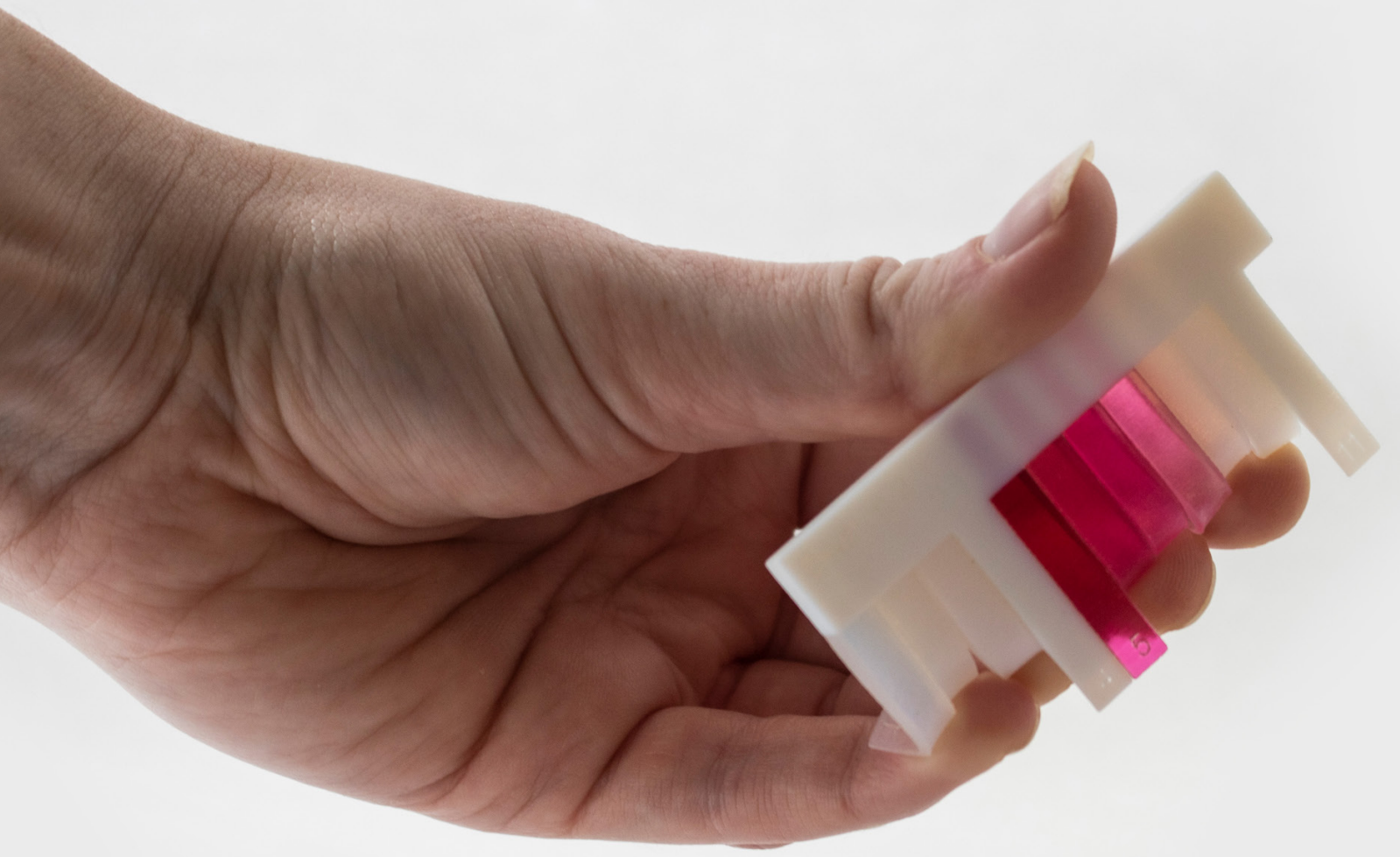


4.3 .3

Materialexploration

Left:

Figure 128. Stratasys $\mathrm{J}^{750^{\mathrm{TM}}}{ }^{\text {Digital Anatomy }}{ }^{\mathrm{TM}}$ 3D-Printer material swatch. 
FILE PREPARATION

\section{Material Swatch File Preparation}
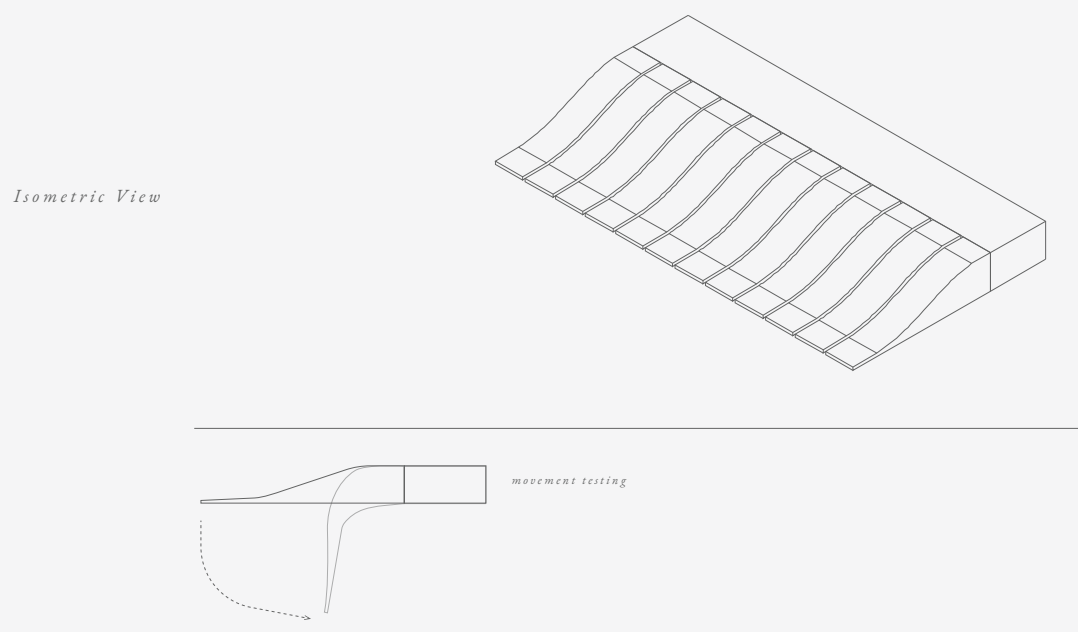

Side profile
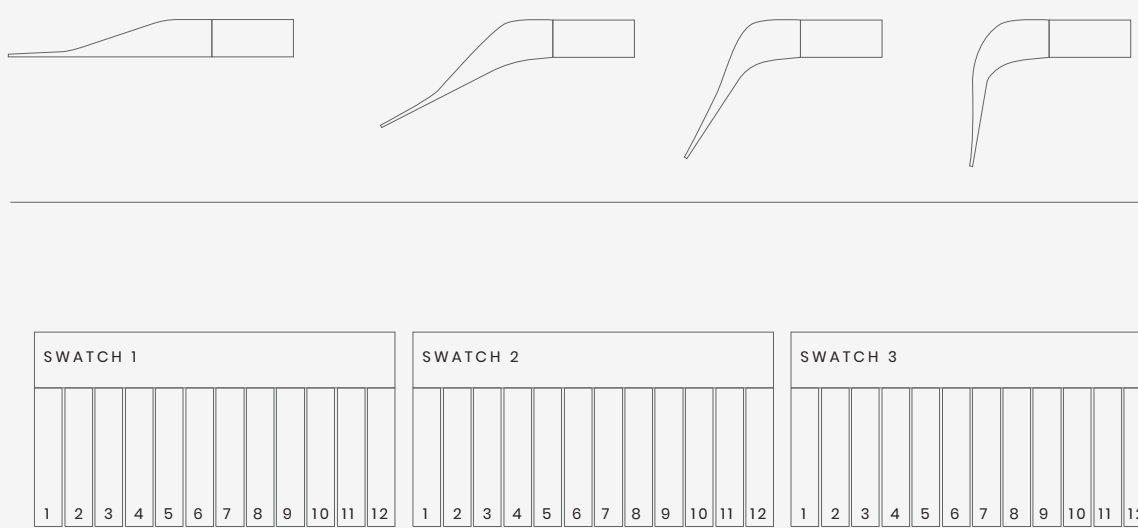

SWATCH 2
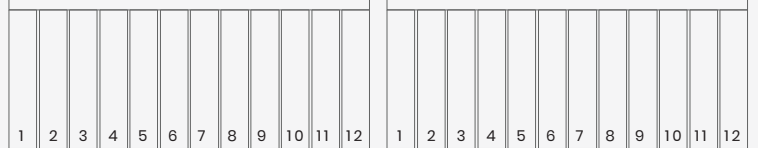

Swatch Files

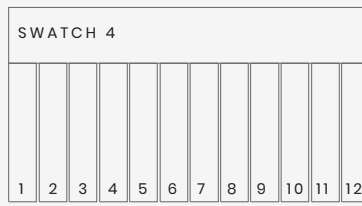

SWATCH 5

SWATCH 7

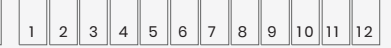

SWATCH 6

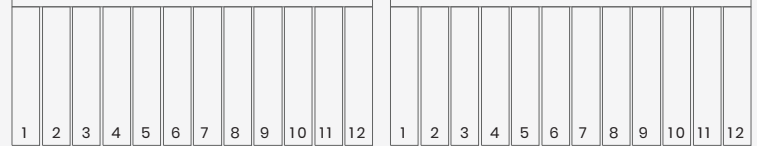

\begin{tabular}{l|l|l|l|l|l|l|l|l|l|l|l}
1 & 2 & 3 & 4 & 5 & 6 & 7 & 8 & 9 & 10 & 11 & 12 \\
\hline
\end{tabular}

Stratasys 3750 m 3D-Printer swatch

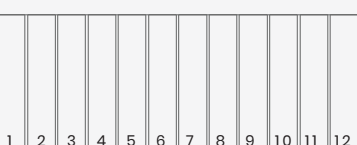




\section{Key for DAP Material Swatches}

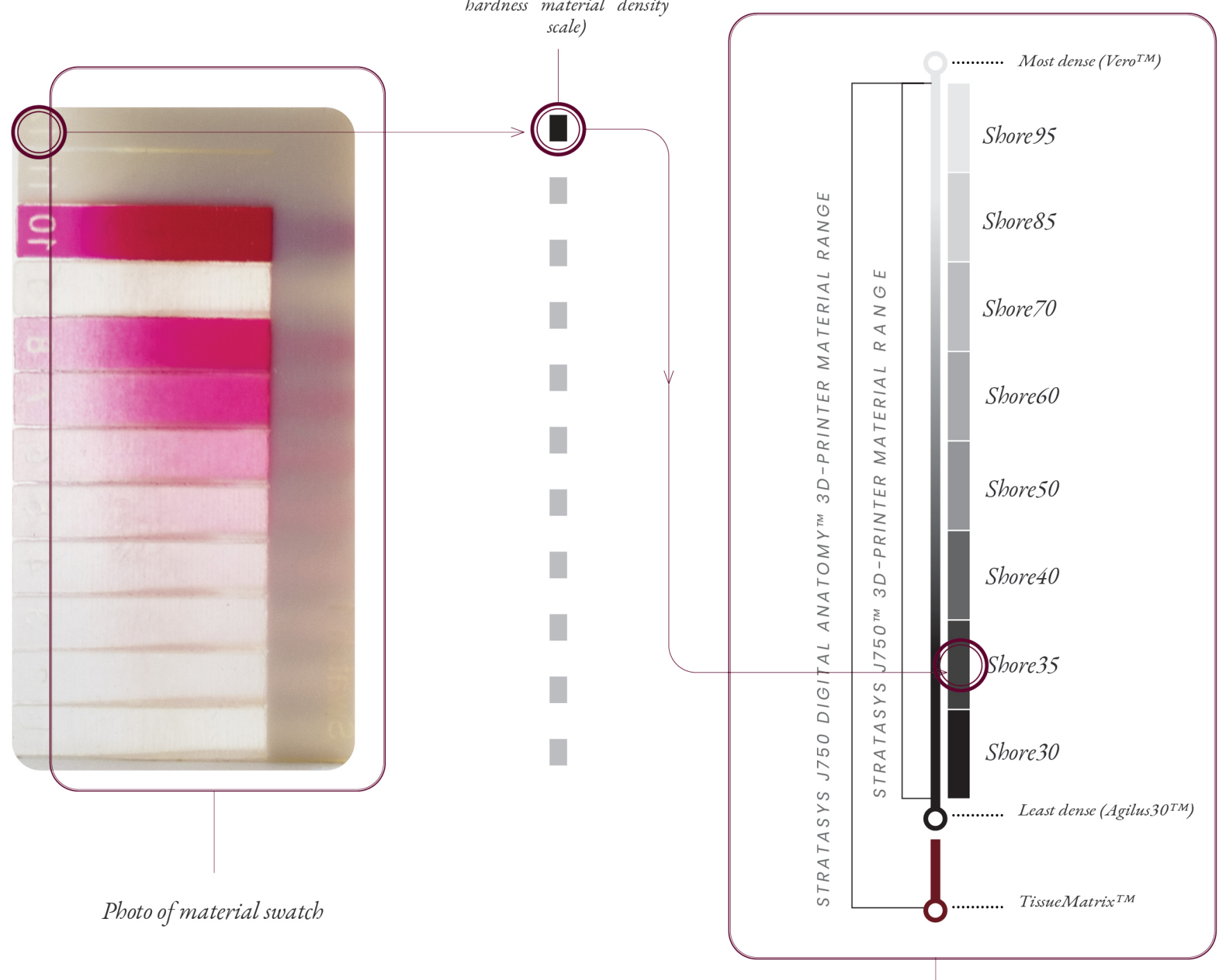

Density of material (colour corresponds to colour on shore hardness material density

SHORE HARDNESS MATERIAL DENSITY SCALE 


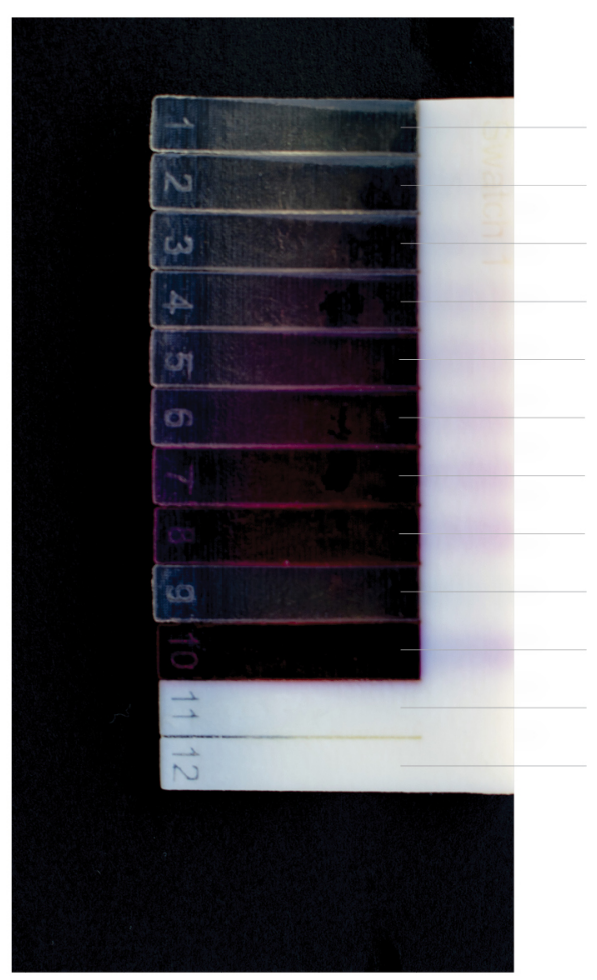

Figure
MATERIAL NAMES

1. Shore $A$ level 30

2. Shore A level 35

3. Shore A level 40

4. Shore A level 50

5. Shore A level 60

6. Shore $A$ level 70

7. Shore A level 85

8. Shore A level 95

9. Agilus30 $30^{\mathrm{TM}}$

10. VeroMagenta-VTM

11. VeroPureWhite ${ }^{\mathrm{TM}}$

12. VeroPureWhite ${ }^{\mathrm{TM}}$

Figure 131. 3D-print \#38 material analysis.

To 3D-print a material swatch on the Stratasys $\mathrm{J} 750^{\mathrm{TM}}$ 3D-Printer based at the School of Design to compare with Stratasys J750 TM Digital Anatomy ${ }^{\mathrm{TM}}$ 3D-printing materials. 


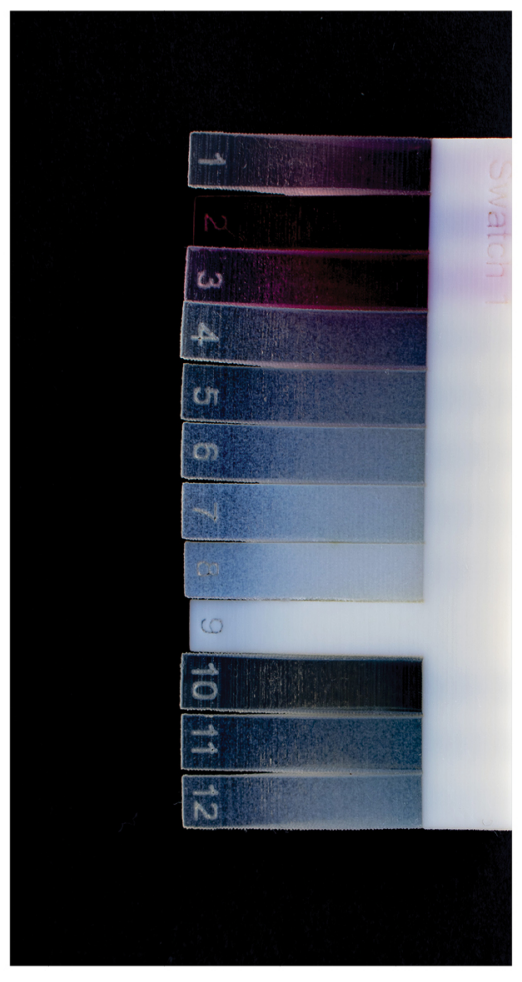

MATERIAL NAMES
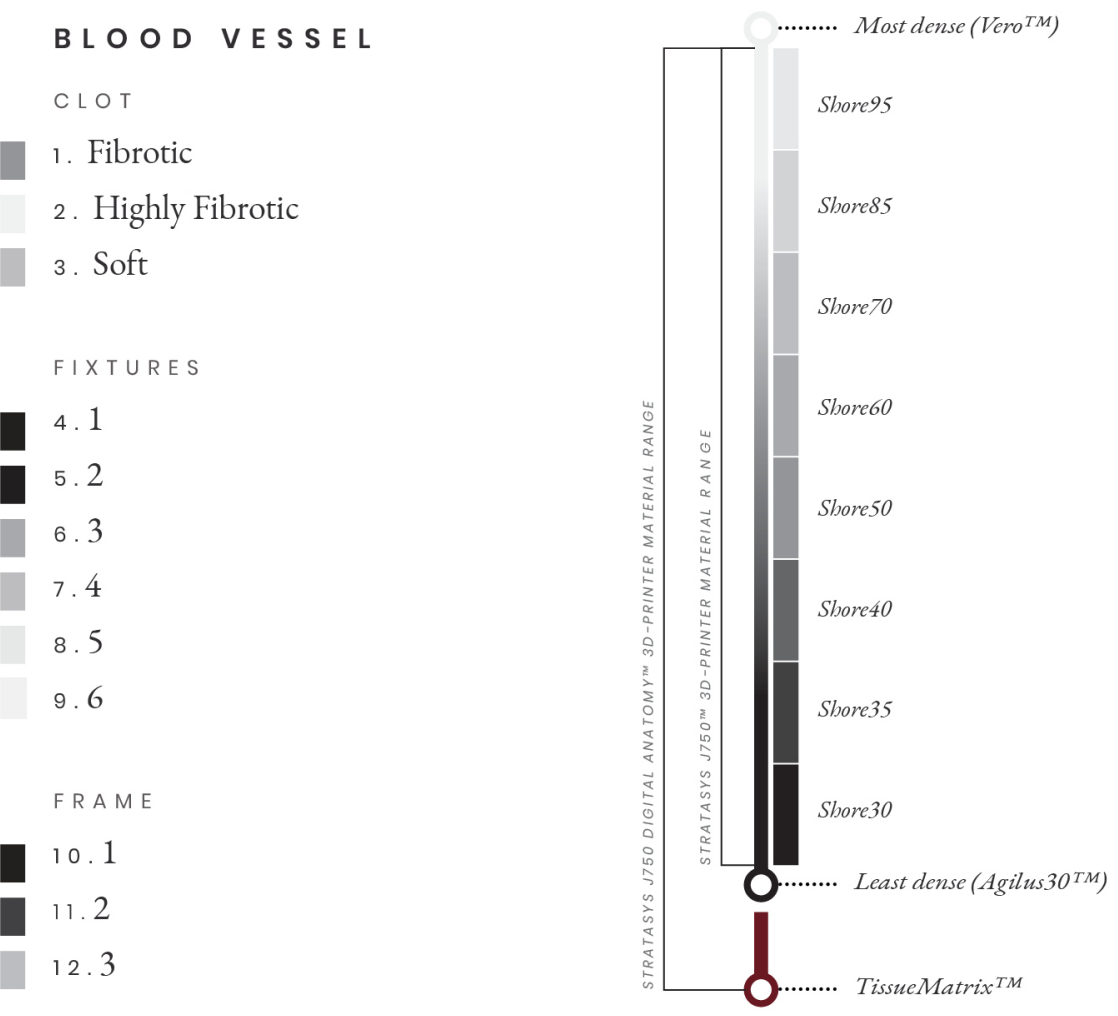

Figure 132. 3D-print \#39 material analysis.
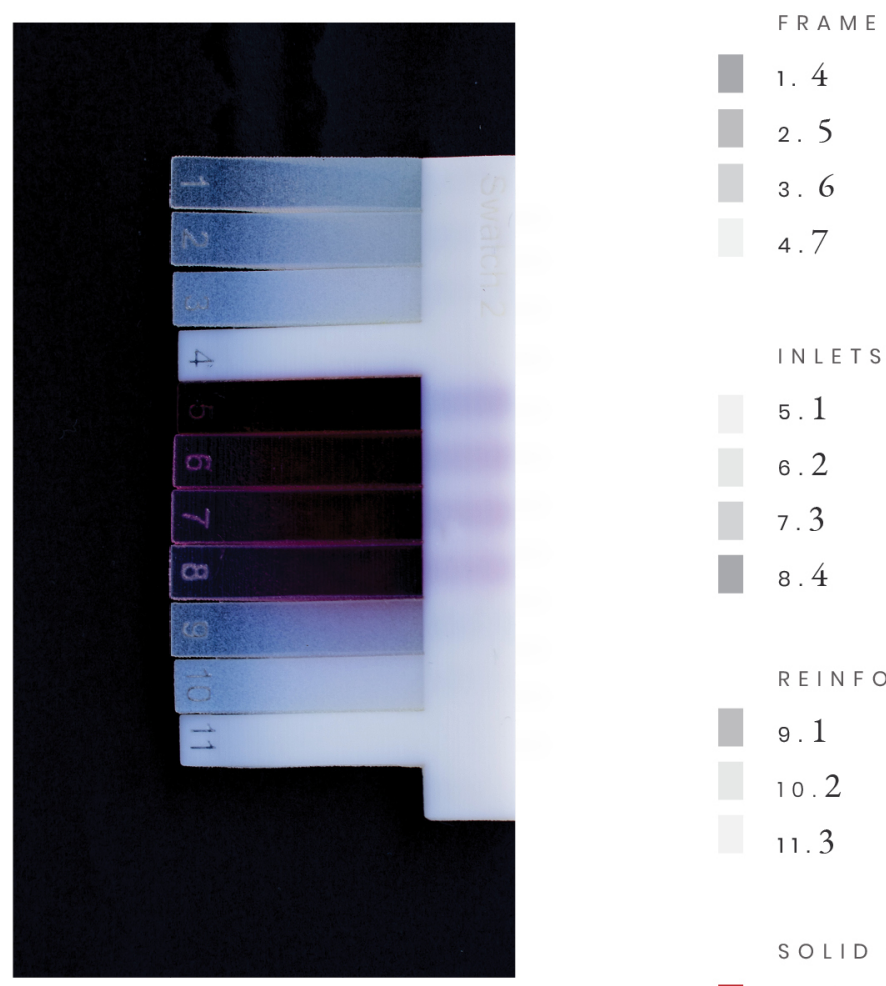

I N LETS

5. 1

6. 2

7. 3

8.4

REINFORCEMENTS

9. 1

10.2

11.3

SOLID T U MOR

fail $12 . \mathrm{A}$

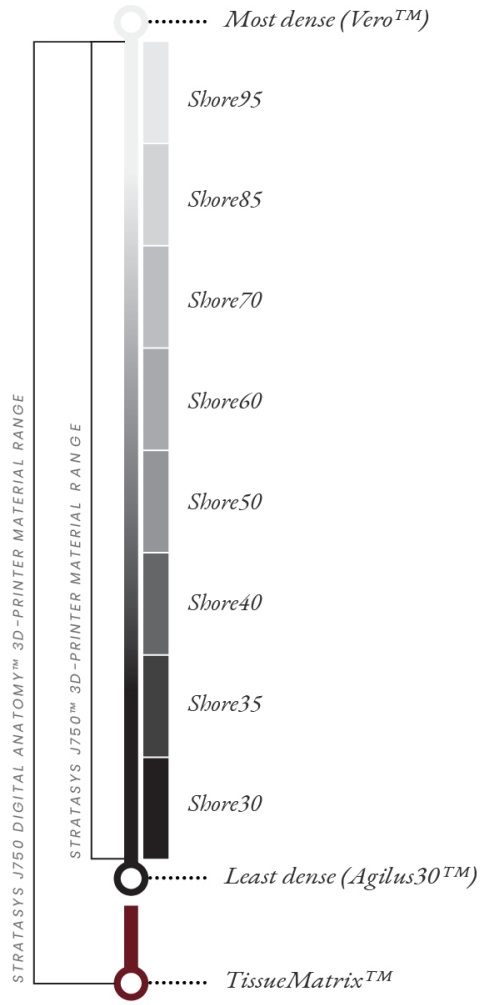

Figure 133. 3D-print \#40 material analysis. 


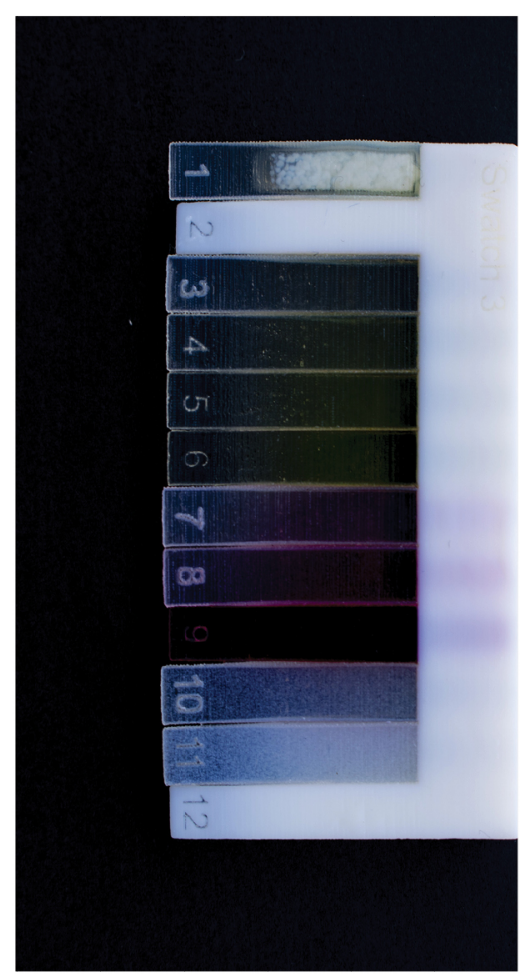

Figure 134. 3D-print \#41 material analysis.
SOLID TUMOR

1. B

2. C

3. D

VALVE ANNULUS

4. Soft

5. Moderately Stiff

6. Stiffened

VALVE LEAFLET

7. Moderately Stiff

8. Stiffened

9. Stiff

VAS CULAR

CALCIFICATION

10. Soft Calcium Deposits

11. Moderately Stiff Calcium Deposits

12. Stiff Calcium Deposits
4.4.3 Case study 3 ........ Most dense (Vero $\left.{ }^{T M}\right)$

Shore95

Shore85

Shore 70

Shore60

Shore 50

Shore 40

Shore 35

Shore 30

Least dense (Agilus30TM)

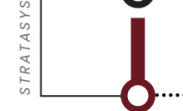

TissueMatrix ${ }^{T M}$

\# 41 D A P swatch

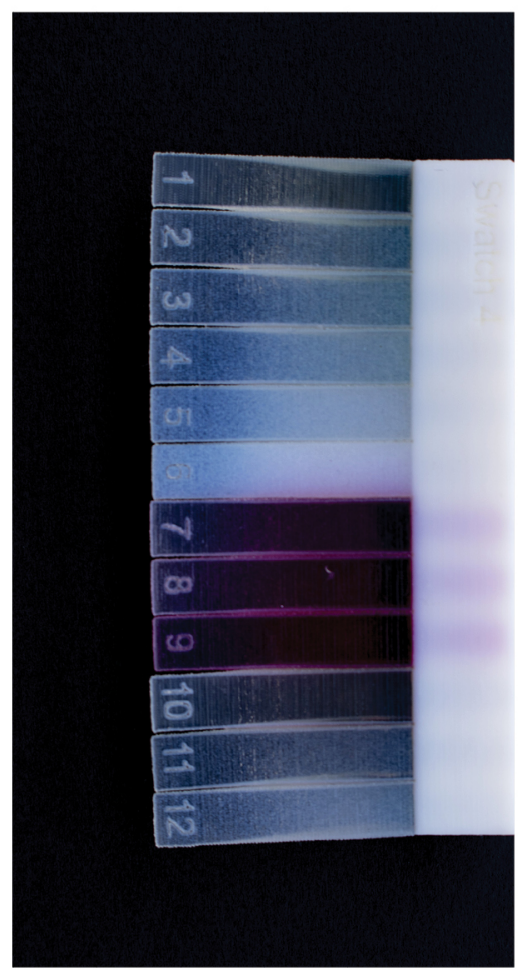

VESSEL WALL

1. Compliant

2. Moderately Compliant

3. Slightly Compliant

4. Low Compliant

5. Semi-Rigid

6. Rigid

GENERAL ANATOMY

DENSE CONNECTIVE TISSUES

7. Soft

8. Rigid

๑. Highly Rigid

HOLLOW INTERNAL ORGANS

10. Compliant

11. Moderately Compliant

12. Slightly Compliant

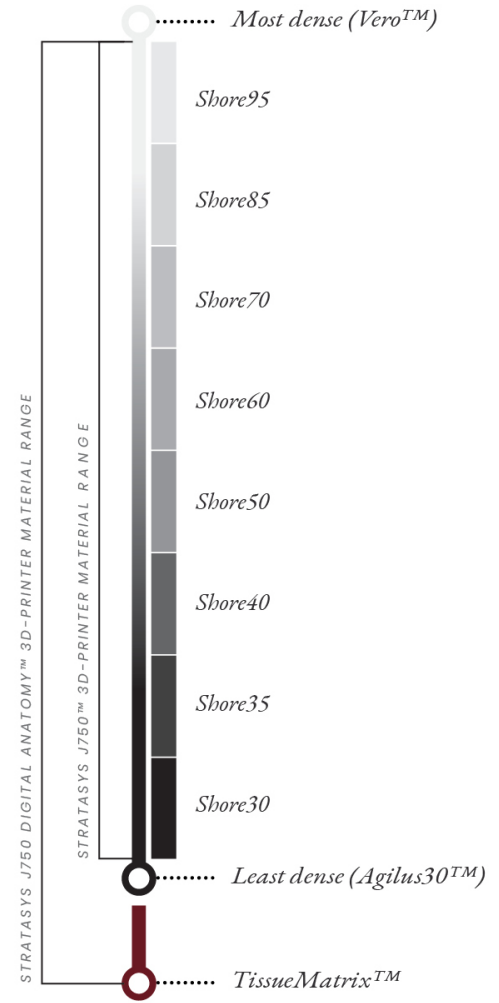

Figure 135. 3D-print \#42 material analysis. 


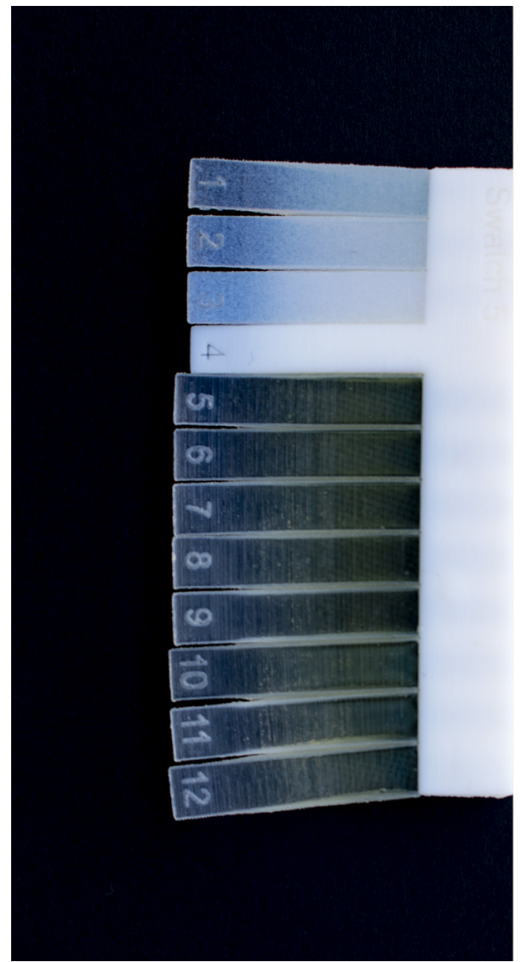

Figure 136. 3D-print \#43 material analysis.

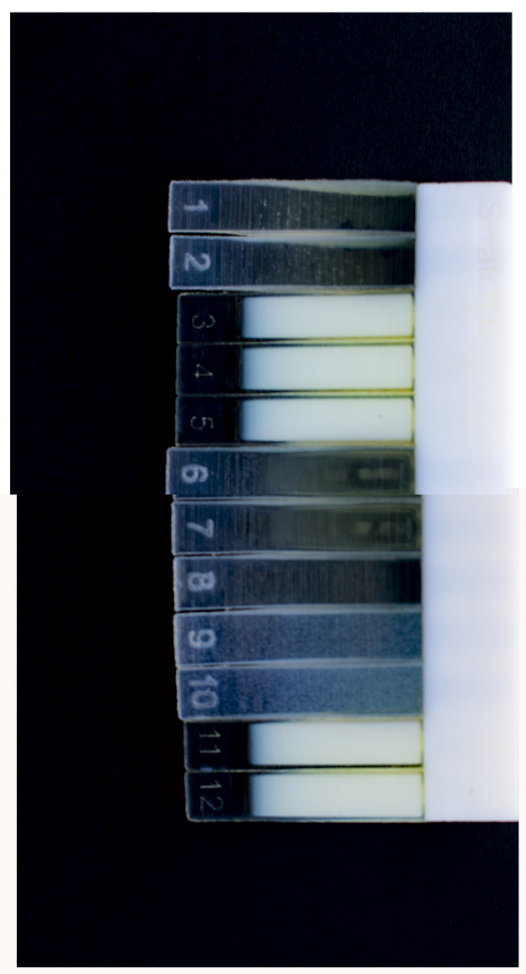

1. Moderately Soft

2. Stiff

GENERAL BONE

3. Porous

4. Slightly Dense

5. Dense

I NTERVERTEBRAL DISKS

6. Normal

7. Degenerated

LI GAMENT

8. Soft

๑. Moderately Stiff

10. Stiff

LONG BONE

11. Miniature

12. Typical

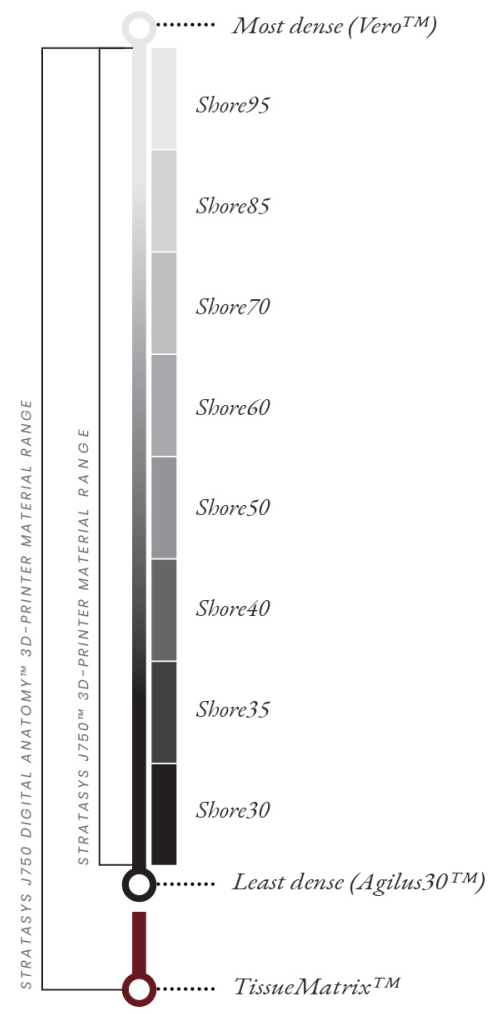

\# $43 /$ DAP Swatch 5

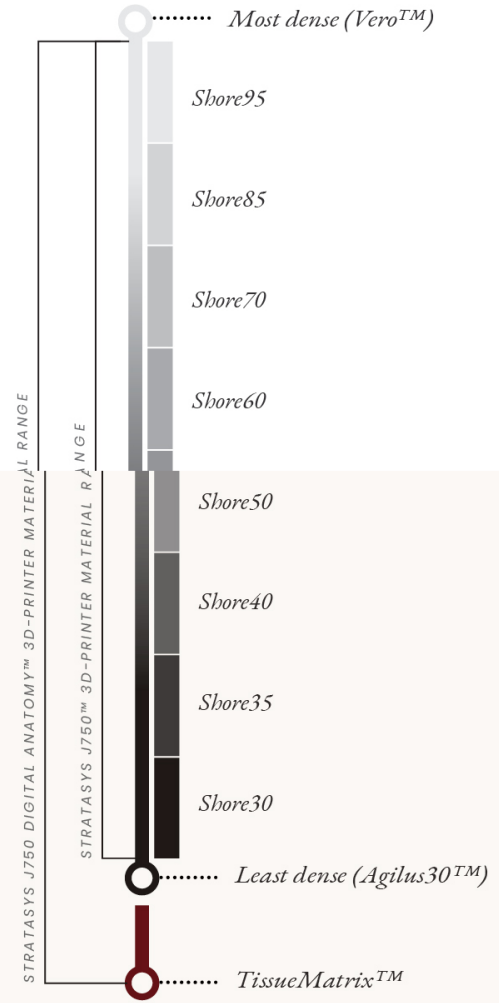

Figure 137. 3D-print \#44 material analysis. 

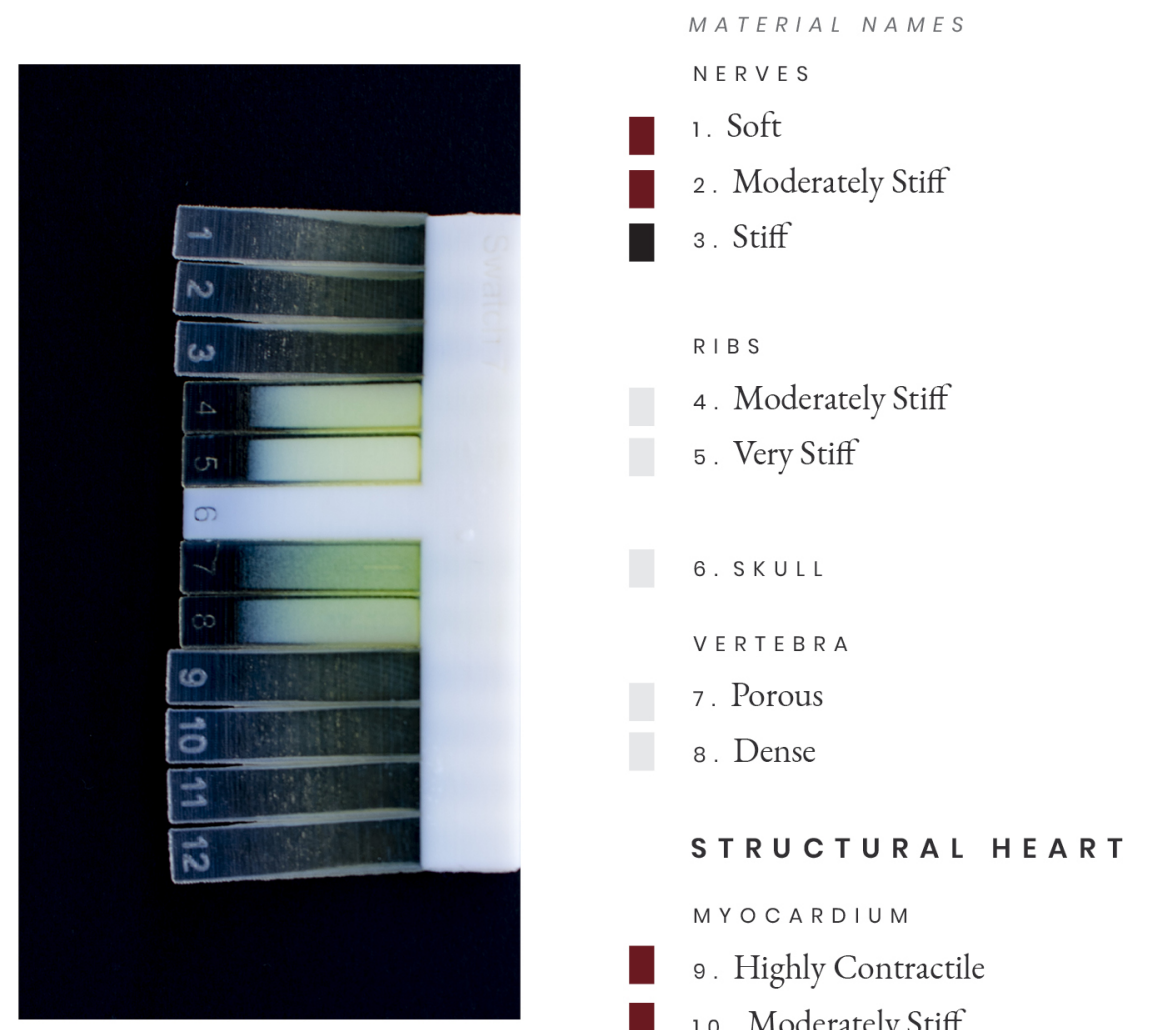

\section{STRU CT URA L HEART}

M Y O C ARDIU M

9. Highly Contractile

10. Moderately Stiff

11. Stiffened

12. Very Stiff

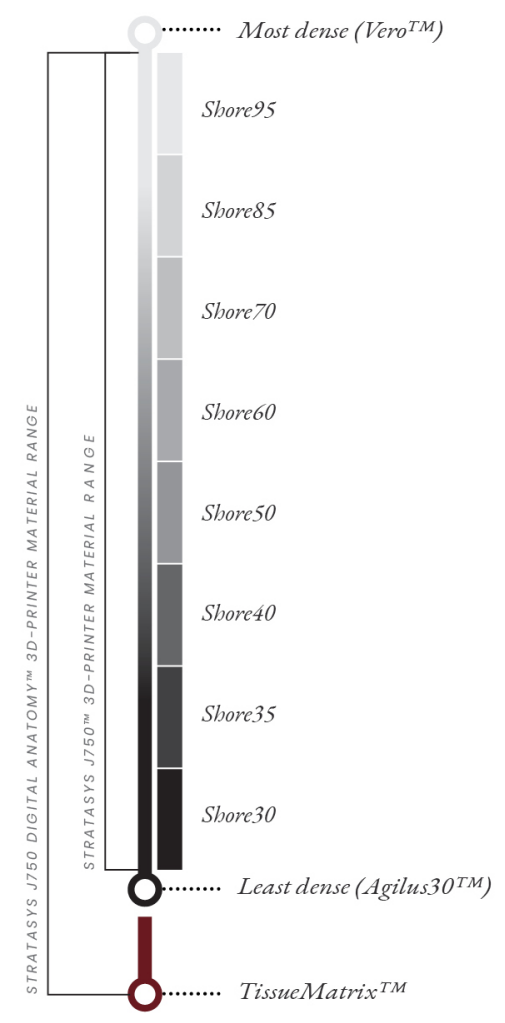

Figure 138. 3D-print \#45 material analysis.

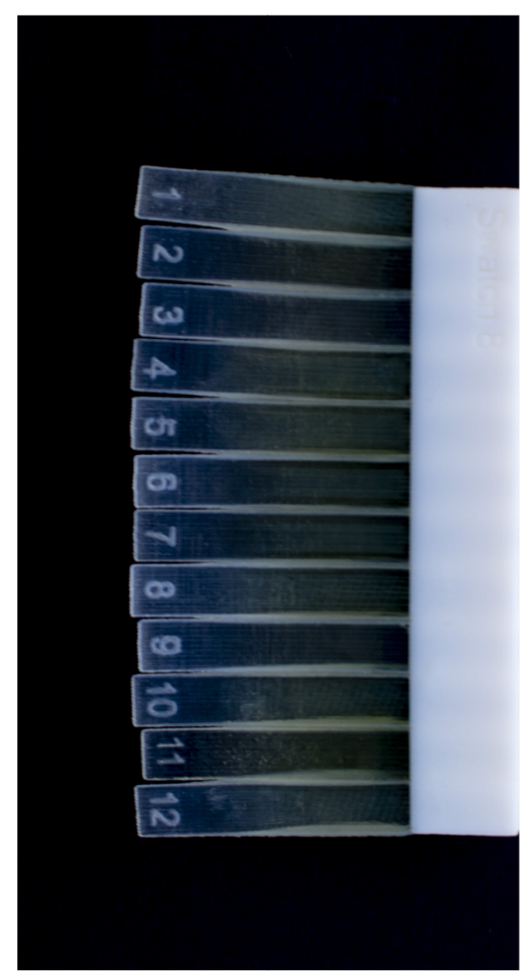

$\square$

VALVE CHORDAE

1. Highly Extensible

2. Extensible

3. Stiffened

\section{EXTR A S}

4. SoftDM_400

5. SoftDM_400

6. SoftDM_400

7. SoftDM_400

1 8. Myocardium_HighlyContractile

๑. GeneralAnatomy_HollowInternalOrgans_Compliant

10. GeneralAnatomy_HollowInternalOrgans_FibreContraction1

11. StructuralHeart_ValveAnnulus_ SoftHealthy

12. StructuralHeart_ValveChordae_HighlyExtensible
\#45/ DAP Swatch 7

Most dense (Vero $\left.{ }^{T M}\right)$

Shore95

Shore 85

Shore 70

Shore60

Shore 50

Shore 40

Shore 35

Shore 30

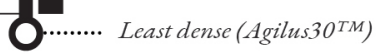

....... TissueMatrix ${ }^{T M}$

Figure 139. 3D-print \#46 material analysis. 


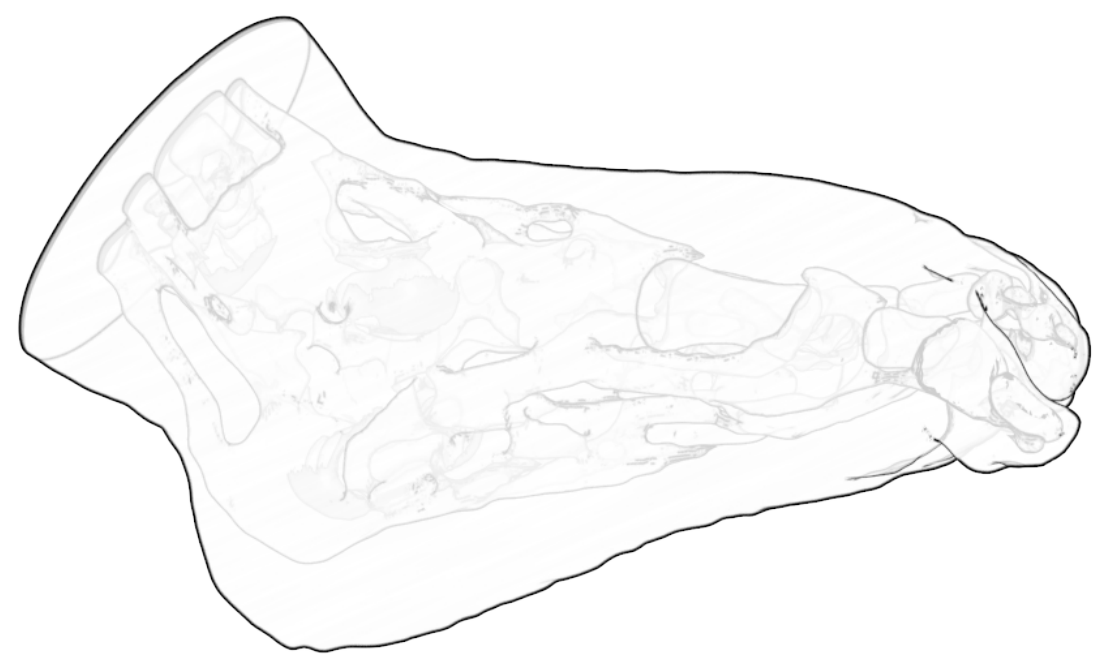

is
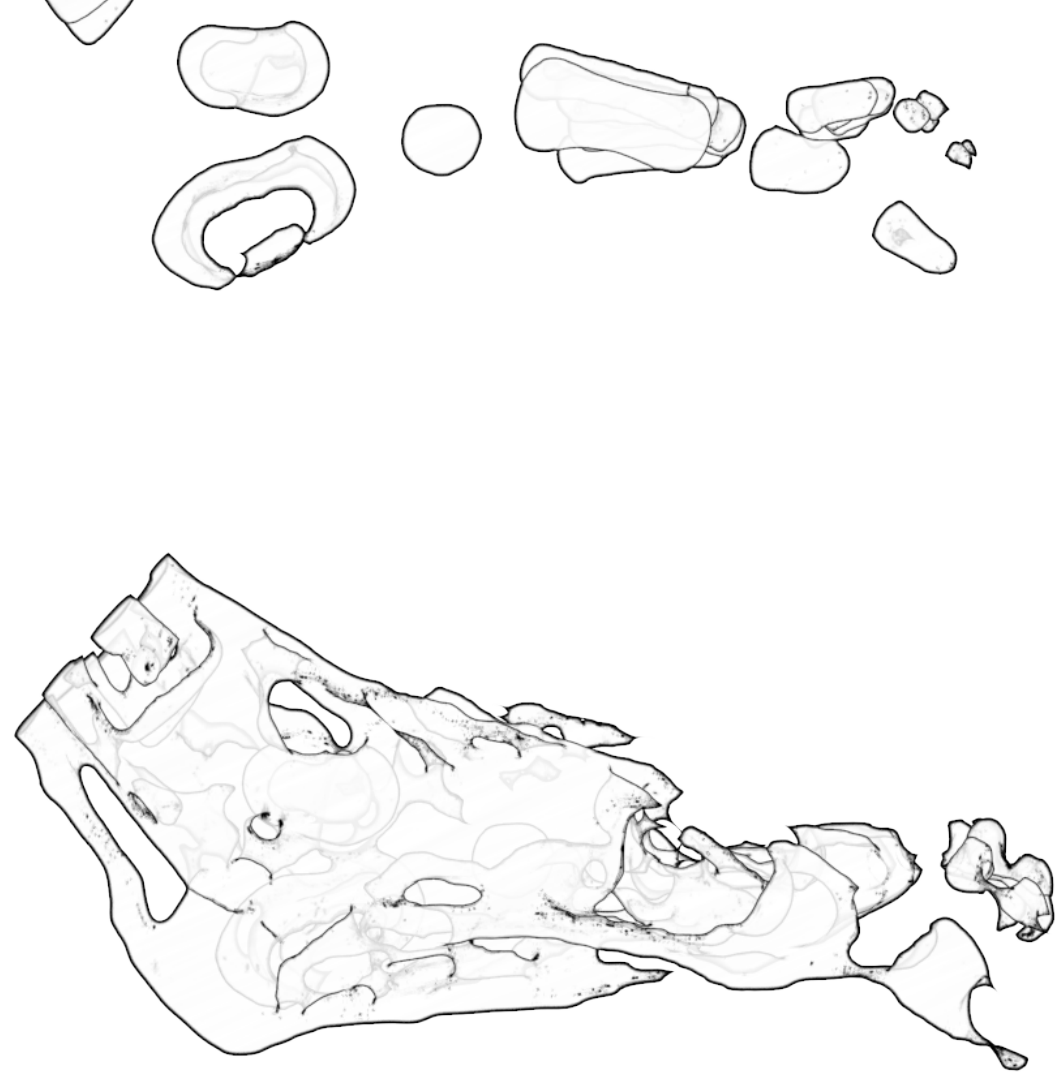
4. 3.4

Design Experimentation

Note: All 3D-prints were coated in SUP706 ${ }^{\mathrm{TM}}$ and cleaned as outlined in Case Study 2 .

Left:

Figure 140. Sketch of neonatal STL (mesh files) used to create the mesh-based neonatal feet on the Stratasys $5750^{\mathrm{TM}}$ Digital Anatomy ${ }^{\mathrm{TM}}$ 3D-Printer. Sketch shows internal "nestled" meshes and cut outs within each STL mesh. 
FOLDER 1

Download volumetric data in a folder

\section{STAGE ONE: Volumetrically Reconstructing Medical Data}

Enter volume reconstruction software.

Edit thresholding settings and values to suit the segmentation needed.

Interpolate images to create a virtual model with smooth surfaces (also known as a voxel model).

Using masking and segmentation
methods extract the anatomy parts
required for the model.
Create three-dimensional geometry
of anatomy.

\section{Make sure the files reflect the area of anatomy correctly. Does the geometry look correct? Are there holes? Does it need a smoothing function applied?}

of anatomy.

Cross check this through medical imaging software volume reconstructions.

Export files of each anatomy part

(eg. bone, soft tissue, fat) as meshes (parts). Each part of anatomy will export as a separate STL file.

\section{FOLDER 2}

Save the files in a folder on the computer.
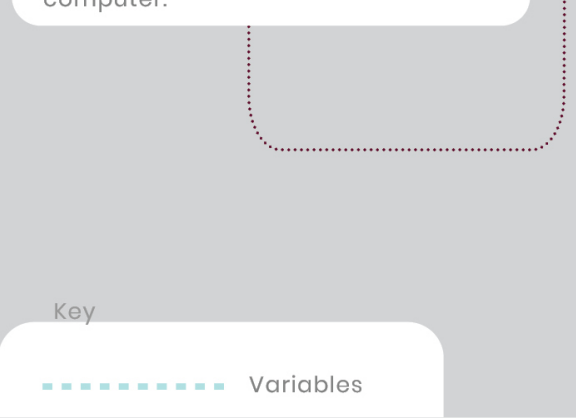

STAGE TWO: Stage Two: Boolean Function (making sure there are no areas of anatomy that intersect)

Import files into Autodesk Netfabb.

- Repair models using the 'Repair Script'

Check the scale.

- Check that the files line up correctly.

- Use the 'Boolean Function' to cut the models from each other. STL assemblies that cross-over at any point can create errors.

- Subtract parts from one another (For example subtract the bone geometry out of the soft tissue geometry).

- One this is completed for each area of anatomy export the Boolean files into a folder and save in FOLDER 3

Check models on Autodesk Meshmixer for any errors.

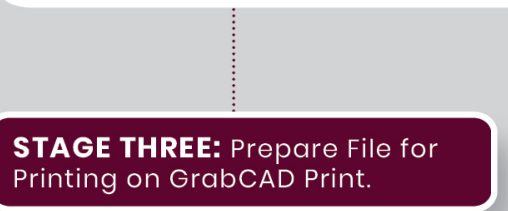

Enter GrabCAD Print and prepare files for 3D-Printing.

Open the 3D-Printer Settings and make sure that the correct 3D-Printer is selected.

- Open the Material Setting and make sure the correct 3D-Printer materials are selected for 3D-Printing.

Import the STL files are an STL assembly

- Assign Printer Materials (drag and drop for each STL part)

Create. OBJZF file and save the print tray

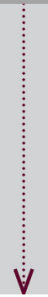

STAGE FOUR: 3D-Print 
To 3D-print a neonatal foot using a traditional mesh-based medical modelling workflow on the Stratasys J750 TM $3 \mathrm{D}$ Printer to serve as a base for comparison when the same model is produced on the Stratasys J750 ${ }^{\mathrm{TM}}$ Digital Anatomy ${ }^{\mathrm{TM}} 3 \mathrm{D}$ -

Printer using new materials.
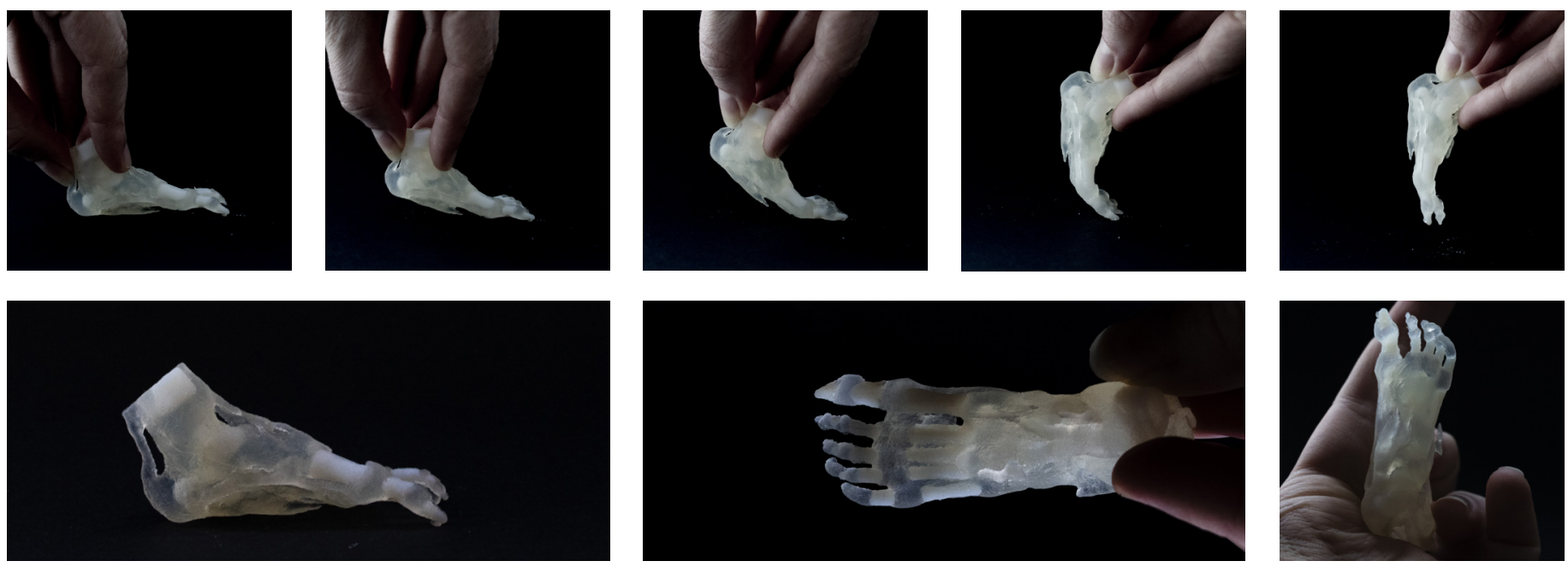

Bone: VeroPureWhite ${ }^{\mathrm{TM}}$

SoftTissue: Agilus $30^{\mathrm{TM}}$

Soft tissue 3D-printed with Agilus30 ${ }^{\mathrm{TM}}$ was flexible,

but not as soft as human flesh. 

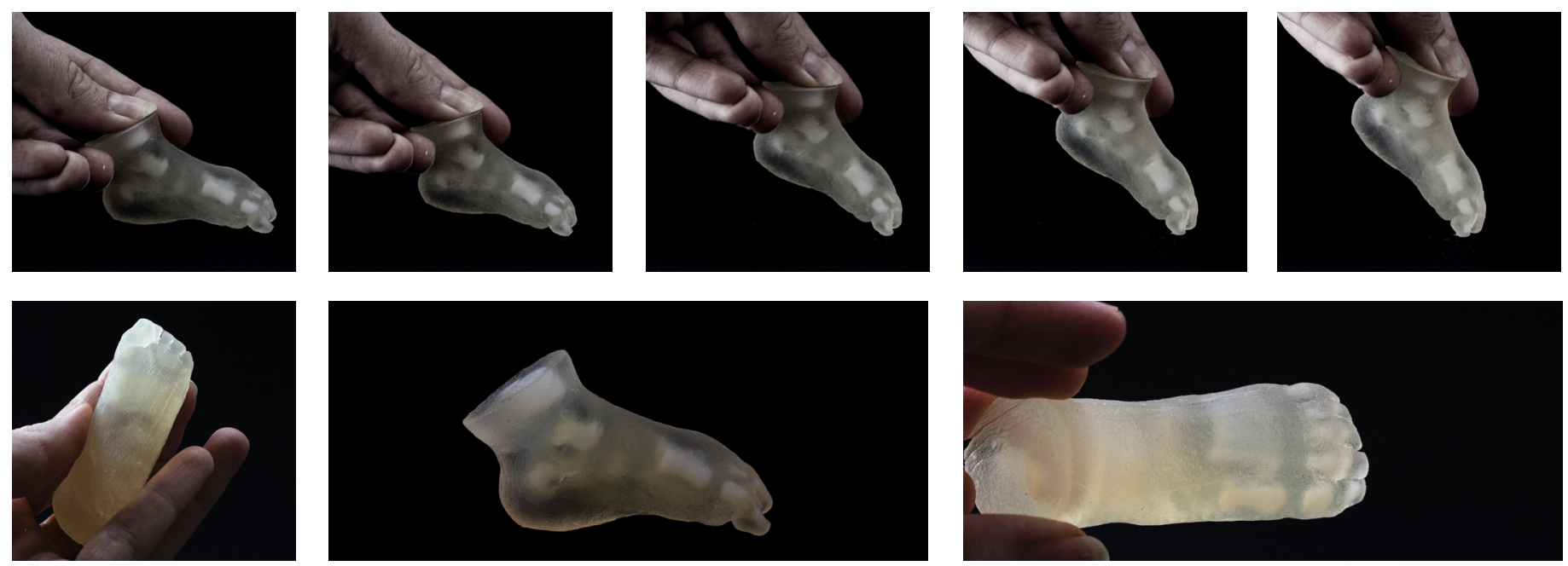

Bone: VeroPureWhite ${ }^{\mathrm{TM}}$

Figure

SoftTissue and Fat: Agilus $30^{\mathrm{TM}}$

Discovery

\#48/ Stratasys J750 тм 3 D-Printer Mesh-based Foot

Agilus $30^{\mathrm{TM}} 3 \mathrm{D}$-printed in large quantities is extremely

rigid. 

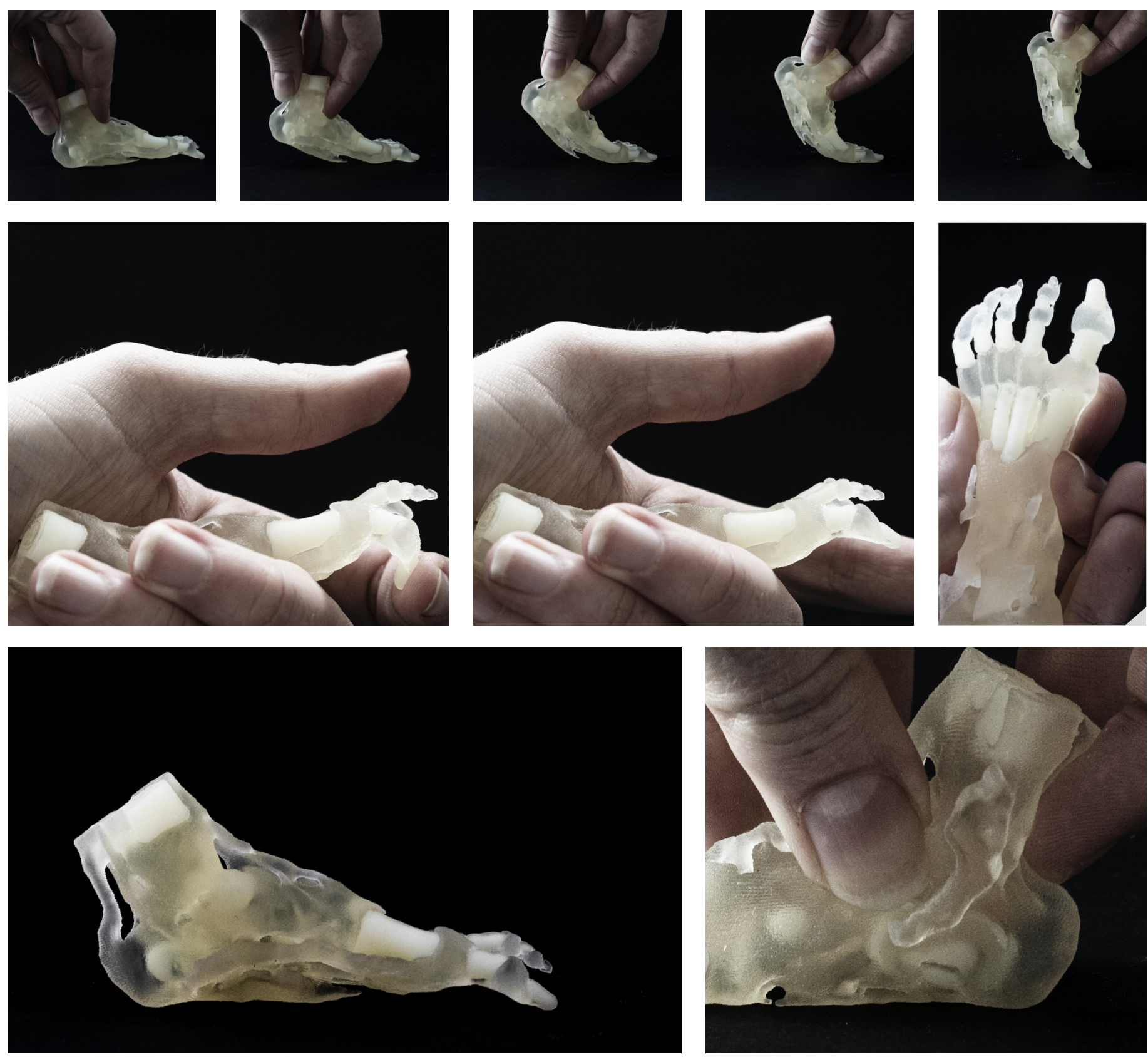

Bone: GeneralBone_Porous

Figure 144. Images of 3D-print \#49.

SoftTissue: SolidInternalOrgans_FibreContraction 1

To 3D-print a neonatal foot using a traditional mesh-based medical modelling workflow (excluding fat and skin) on the the Stratasys J750 ${ }^{\mathrm{TM}}$ Digital Anatomy ${ }^{\mathrm{TM}}$ 3DPrinter to test and compare novel Digital Anatomy ${ }^{\mathrm{TM}}$ materials.
The most life-like and realistic model materials 3D-printed in the research. Is limited because the model contains no gradients in density and colour. 

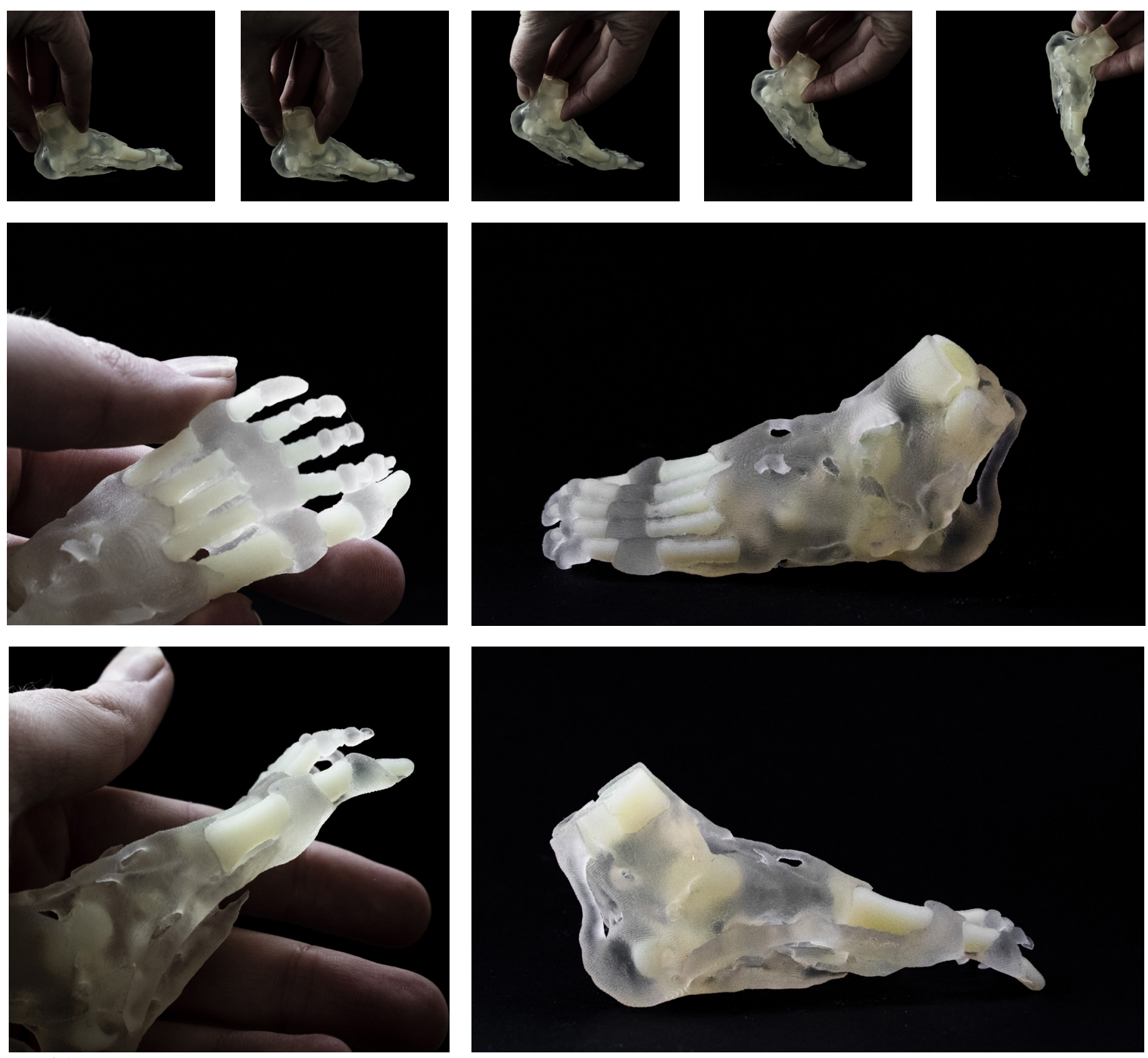

Bone: Ribs_ModeratelyStiff

Figure 145. Images of 3D-print \#50.

Soft Tissue: Ligament_Soft

\# $50 /$ DAP Foot 2

without fat and skin

Similar stiffness to Agilus30 3D-print \# 47

(not as soft as 3D-print \# 49). 

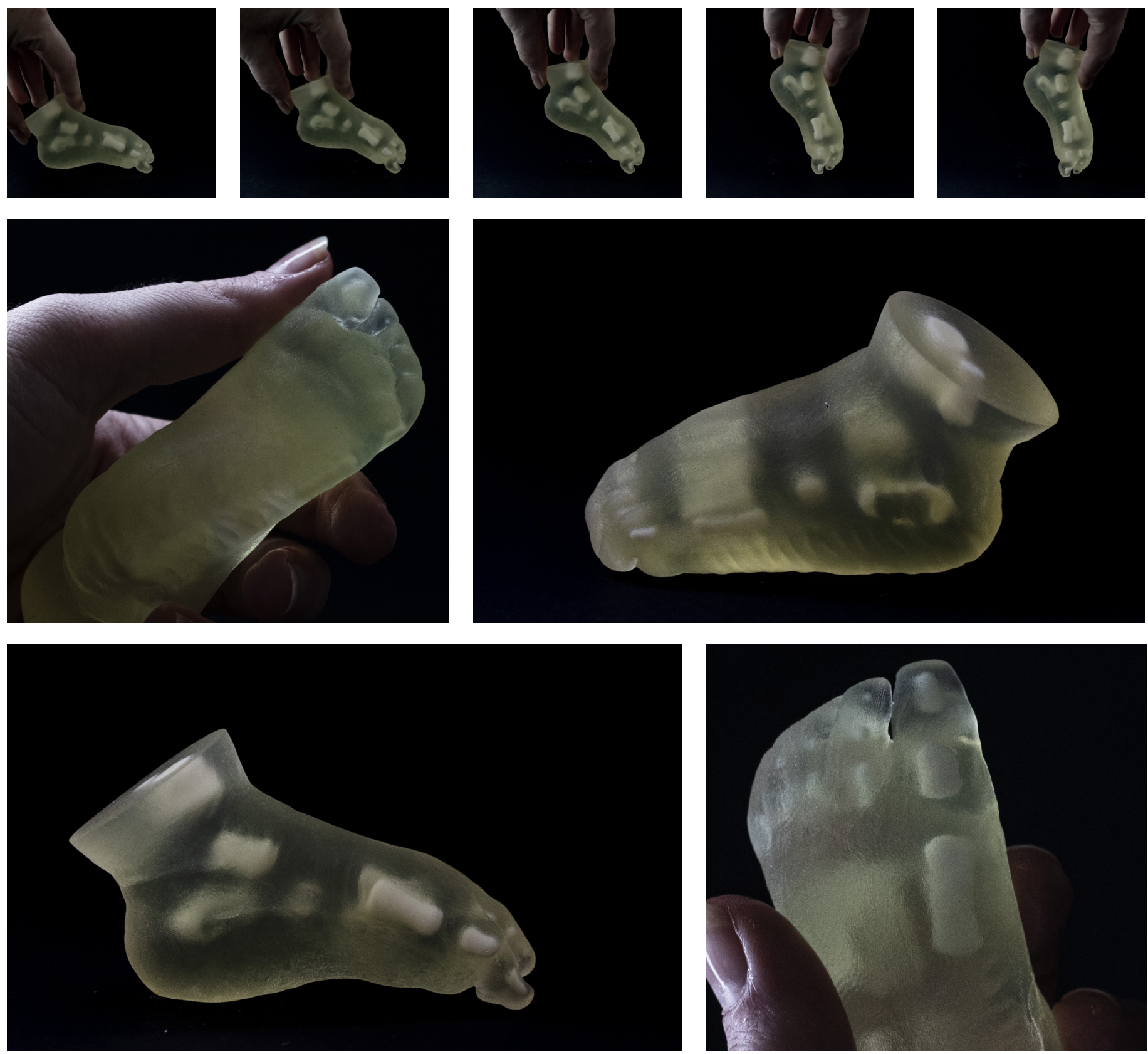

Bone: GeneralBone_SlightlyDense

SoftTissue: Ligament_Soft

Fat: StructuralHeart_ValveAnnulus_SoftHealthy

Aim (3D-print \#51, \#52 \& \#53)

To 3D-print a neonatal foot using a tradi-

The resulting 3D-print was rigid with a yel-

tional mesh-based medical modelling work-

low tint.

flow (including fat and skin) on the the

Stratasys J750 ${ }^{\mathrm{TM}}$ Digital Anatomy ${ }^{\mathrm{TM}} 3 \mathrm{D}$ -

Printer to test and compare novel Digital

Anatomy ${ }^{\mathrm{TM}}$ materials. 

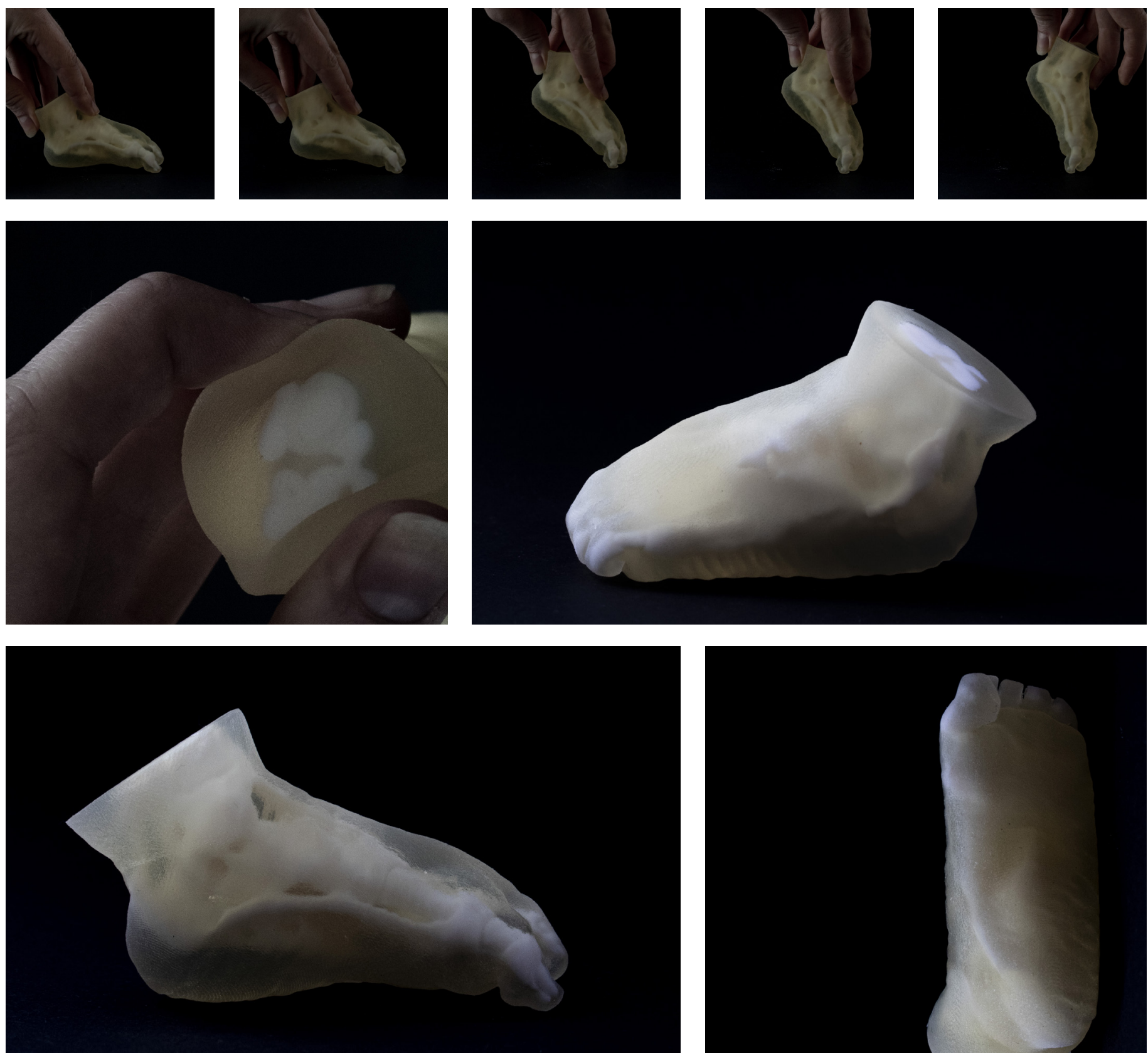

Bone: GeneralBone_Porous

SoftTissue: VeroPureWhite ${ }^{\mathrm{TM}}$

Fat: VeroPureWhite ${ }^{\mathrm{TM}}$

Discover

A mistake was made during GRABCAD processing and the internal structure $3 \mathrm{D}$ printed in VeroPureWhite ${ }^{\mathrm{TM}}$. The external soft material was substantially less dense and more life-like than Agilus30 $30^{\mathrm{TM}}$ in 3Dprint \#48. 

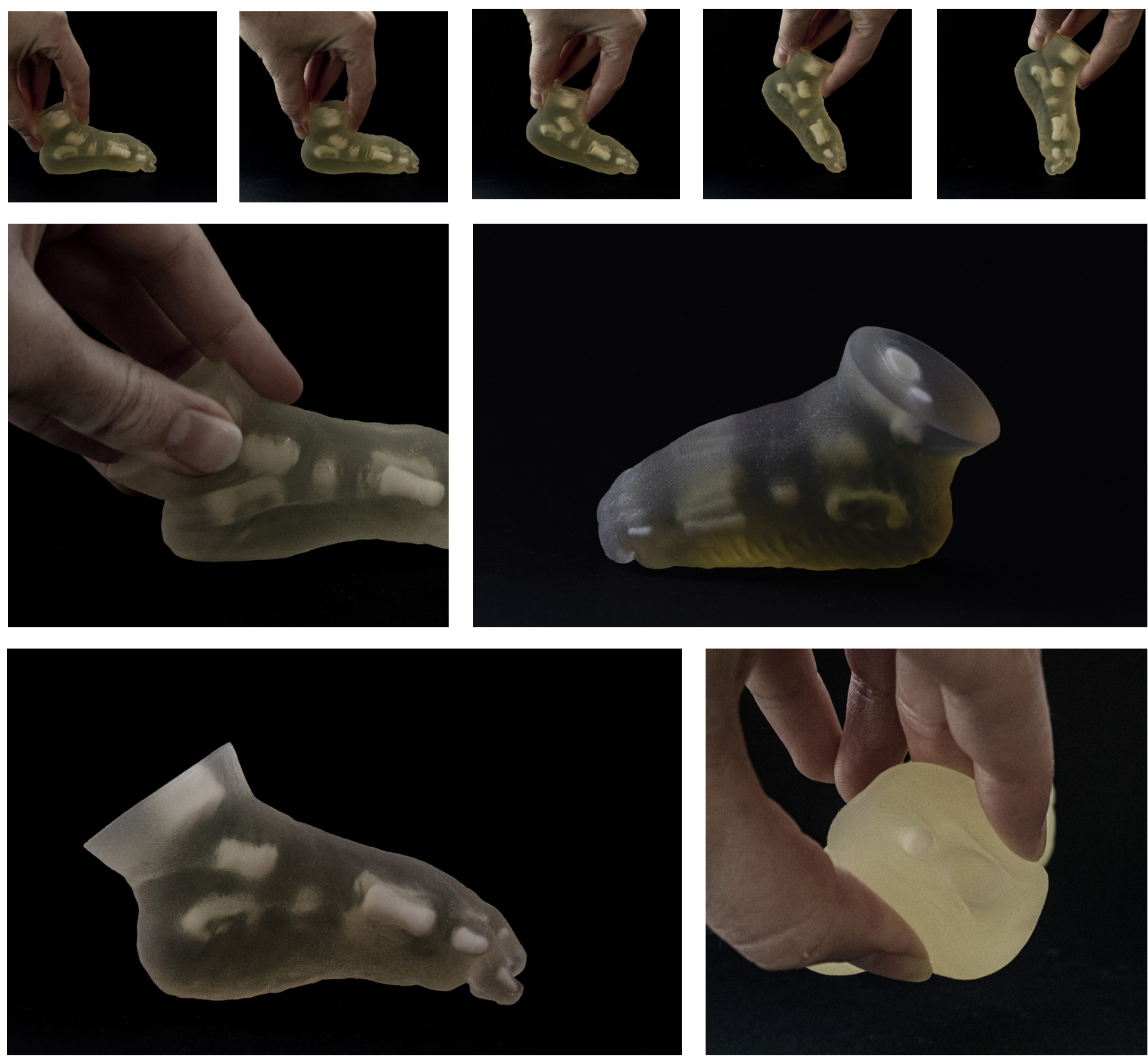

Bone: GeneralBone_Porous

SoftTissue: Myocardium_HighlyContractile

Fat: SoftDM_400

Discovery

This was the softest and most life-like 3Dprint using the complete neonatal foot file. Unlike 3D-print \#36, the model could not be cut open revealing layers of skin and fat due to limitations of mesh-based 3D-printing. 

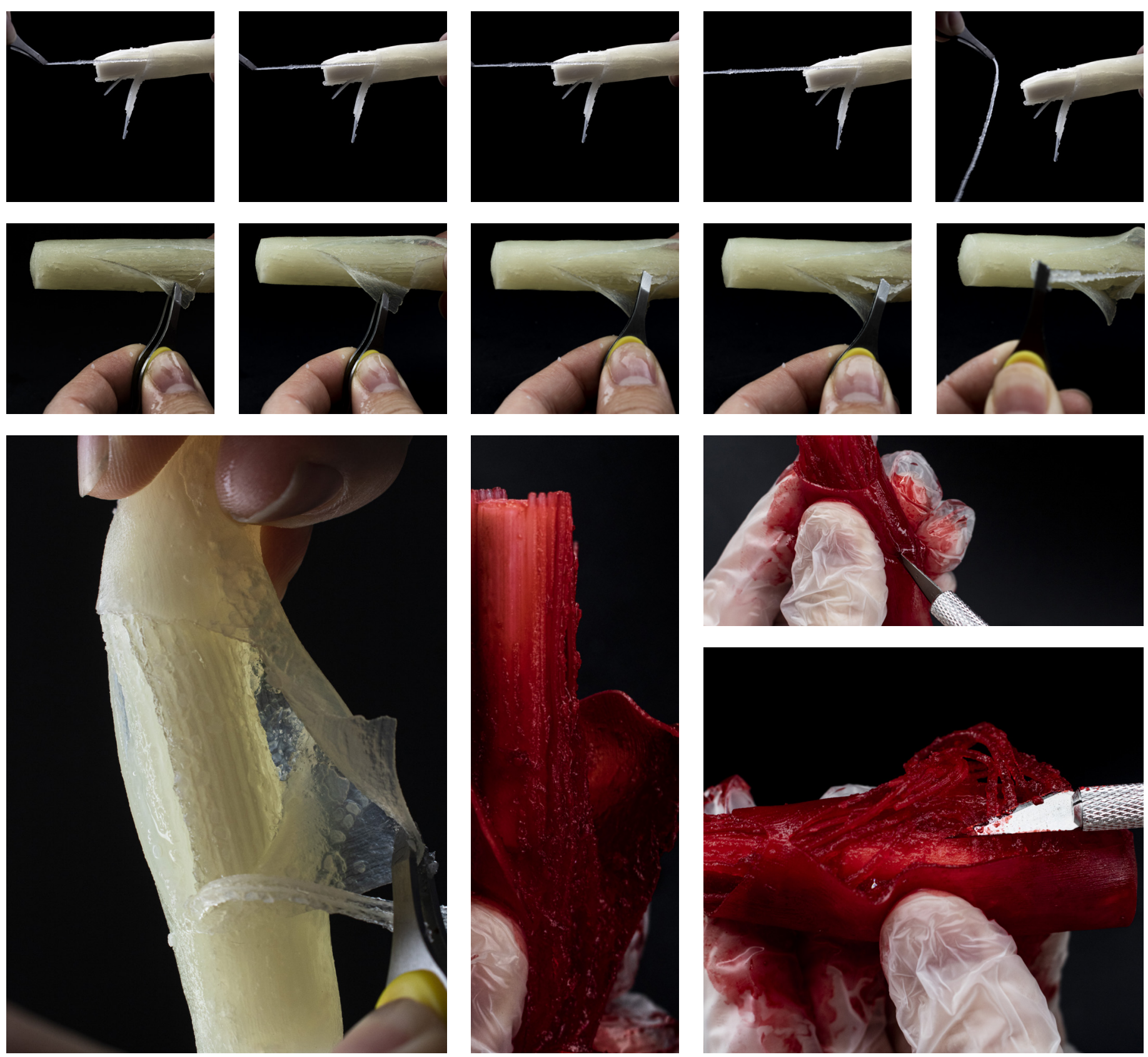

Figure 149. Images of 3D-print \#54.

To 3D-print a CAD modelled test swatch with GelMatrix ${ }^{\mathrm{TM}}$, bone, skin, and thin strips of fibrous material for simulation and skills acquisition purposes.
The resulting 3D-print was easy to interact with. The fibres were able to be pulled out and the skin could be cut and peeled off. 


\section{3 .5}

Evaluation and Reflection

Without a doubt, the Stratasys J750 ${ }^{\text {TM }}$ Digital Anatomy ${ }^{\text {TM }}$ 3D-printing materials are softer and the materials 'bounce back' (elasticity) and return to their original state at a faster pace than Stratasys $\mathrm{J} 750^{\mathrm{TM}}$ 3D-printing materials. These new material qualities mean that 3D-prints produced are more realistic. This mitigates some of the frustrations caused by Stratasys J750 ${ }^{\mathrm{TM}}$ 3D-Printer.

MOVING FORWARD

The next step of this Case Study considered the advantages of all three Case Studies to develop a hypothetical, yet realistic, bitmap-based medical modelling GrabCAD add-on. The workflow includes the realistic colour qualities of Case Study 1, the bitmap-based 3D-printing workflow of Case Study 2 and the Stratasys J750 ${ }^{\mathrm{TM}}$ Digital Anatomy ${ }^{\mathrm{TM}} 3 \mathrm{D}$-printing materials presented in Case Study 3.

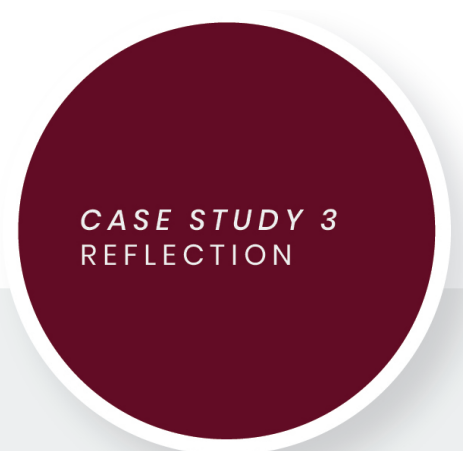

Case Study 3 opened up the possibilities for future explorations. It would be interesting to consider using the Voxel Print Utility on the Stratasys $750^{\mathrm{TM}}$ Digital Anatomy ${ }^{\mathrm{TM}}$ 3D-Printer and developing a system to support this new workflow. 


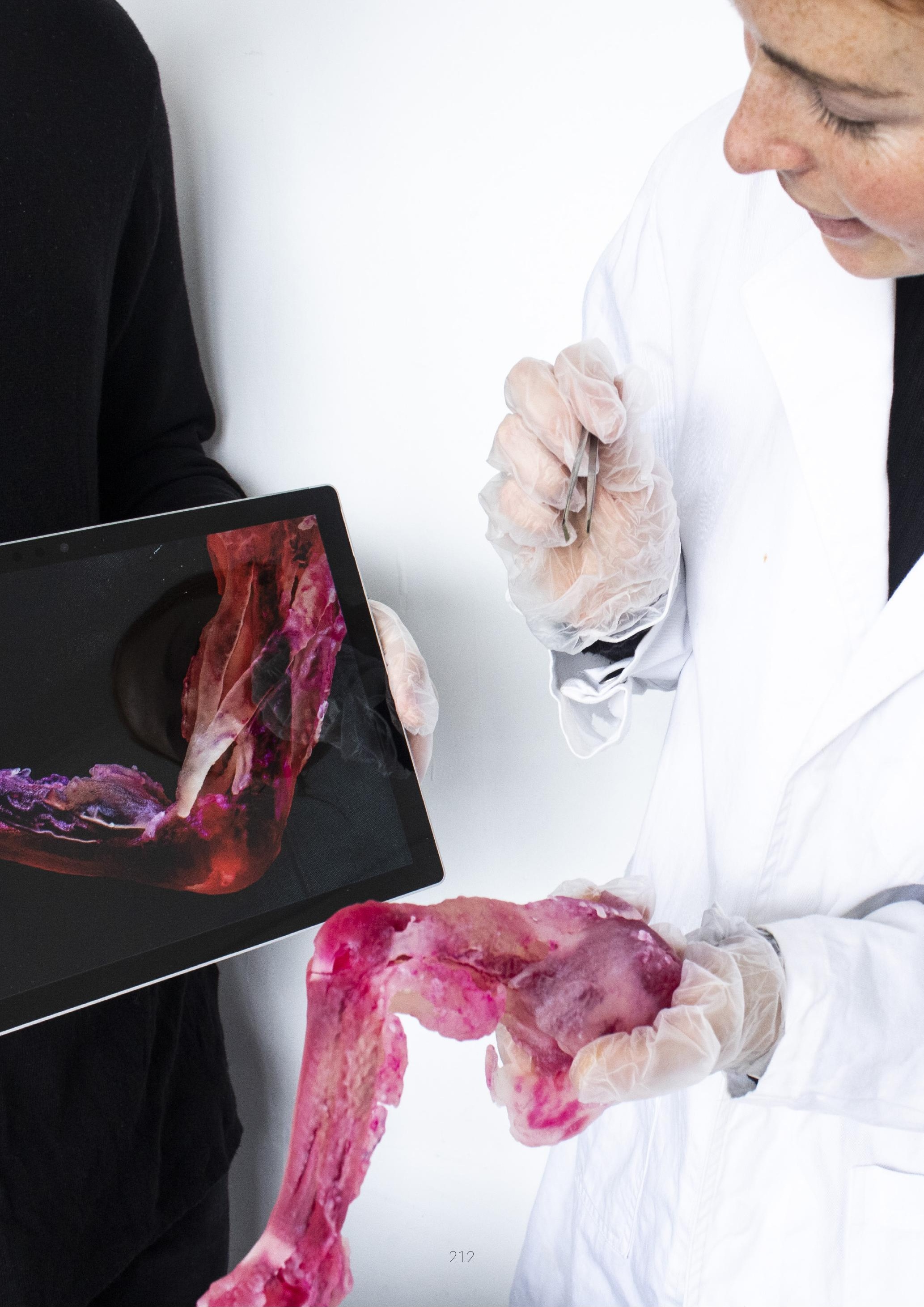



STEP ONE

Acquiring patient-specific medical data through data acquisition (CT scan).
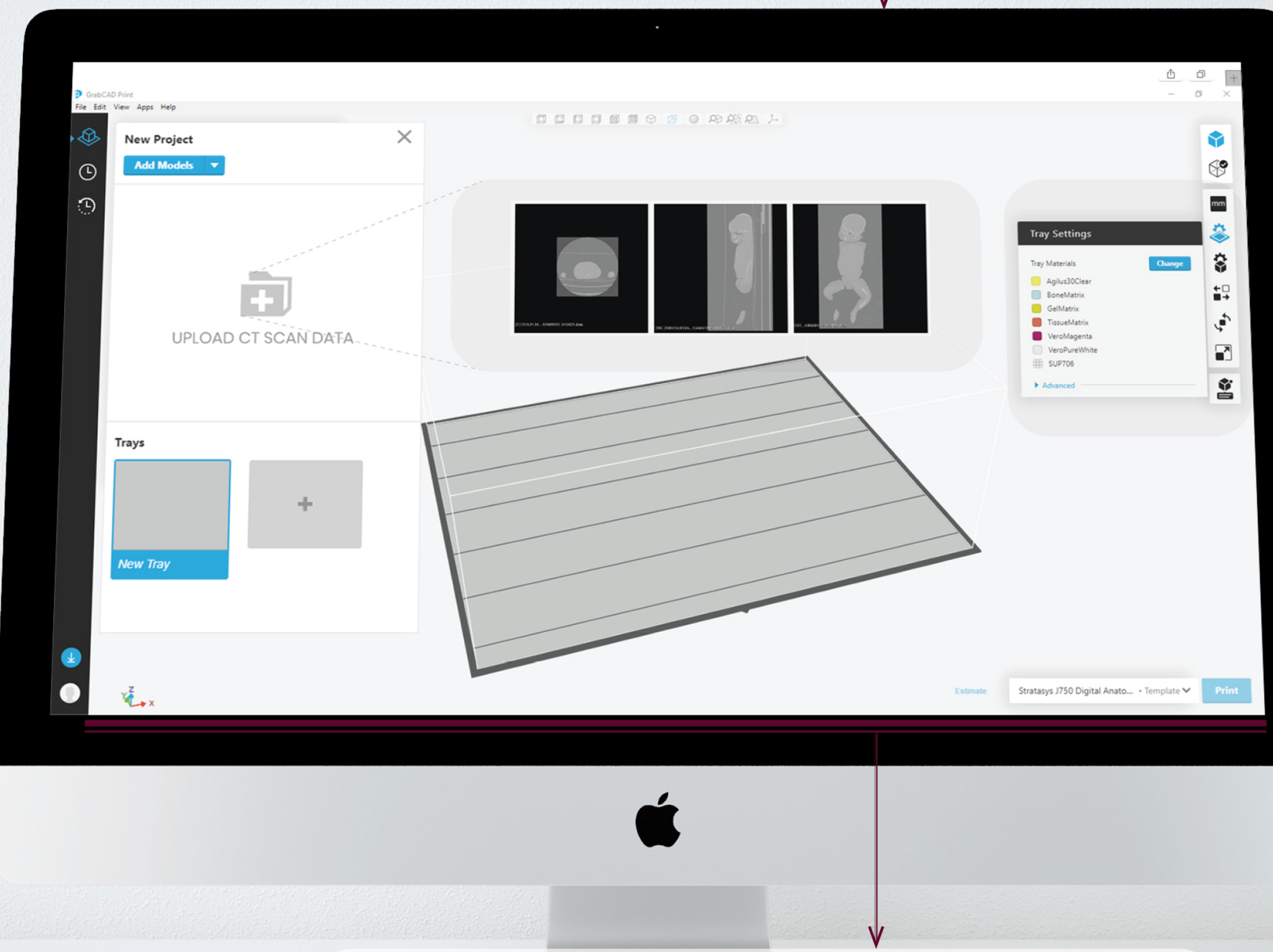

STEP TWO

Manual Step: Uploading the patient specific medical data to GrabCAD.

Automated Step: Scan smoothing and interpolation of images to create a virtual volumetric voxel reconstruction of the scanned patient.

A

Figure 152. (A-D) A developed streamlined medical modelling workflow concept. The images present an idea for a software plug-in for GrabCAD Print using the Stratasys $\mathrm{J}^{750^{\mathrm{TM}}}$ Digital Anatomy ${ }^{\mathrm{TM}}$ 3D-Printer using newly developed materials. These images have been designed using open-source resources from https://www.mockupworld.co/free/workspace-imac-mockup-2/. 

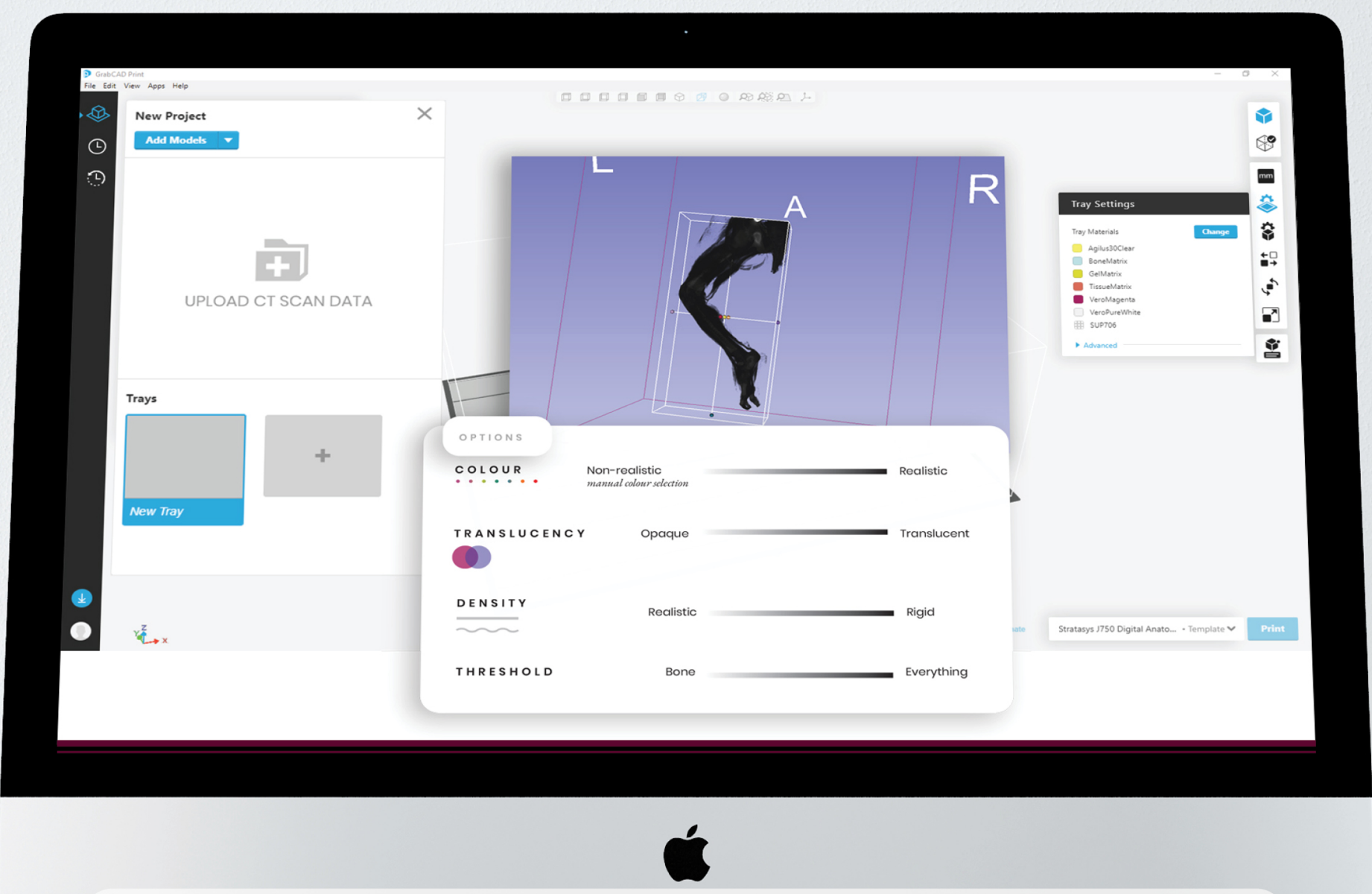

STEP THREE

Manual Step: In this step, the user can crop the area of anatomy. Following this, the user has the ability to manipulate the model's colour, translucency, density, and threshold.

Automated Step: The software automatically figures out the attenuation properties of the scan and can automatically update the volumetric reconstruction according the preference that has been adjusted. Then, the software will digitally halftone and interpolate the bitmap images with the native Stratasys $\mathrm{J}_{750^{\mathrm{TM}}}$ Digital Anatomy ${ }^{\mathrm{TM}}$ 3Dprinting materials at the native 3D-printer $\mathrm{z}$ resolution. 3D-printer materials would include TissueMatrix ${ }^{\mathrm{TM}}$ (in all CMYK colours) and BoneMatrix ${ }^{\mathrm{TM}}$ (in all CMYK colours) so that full-colour and realistic anatomical density can be achieved in a single 3D-print. 


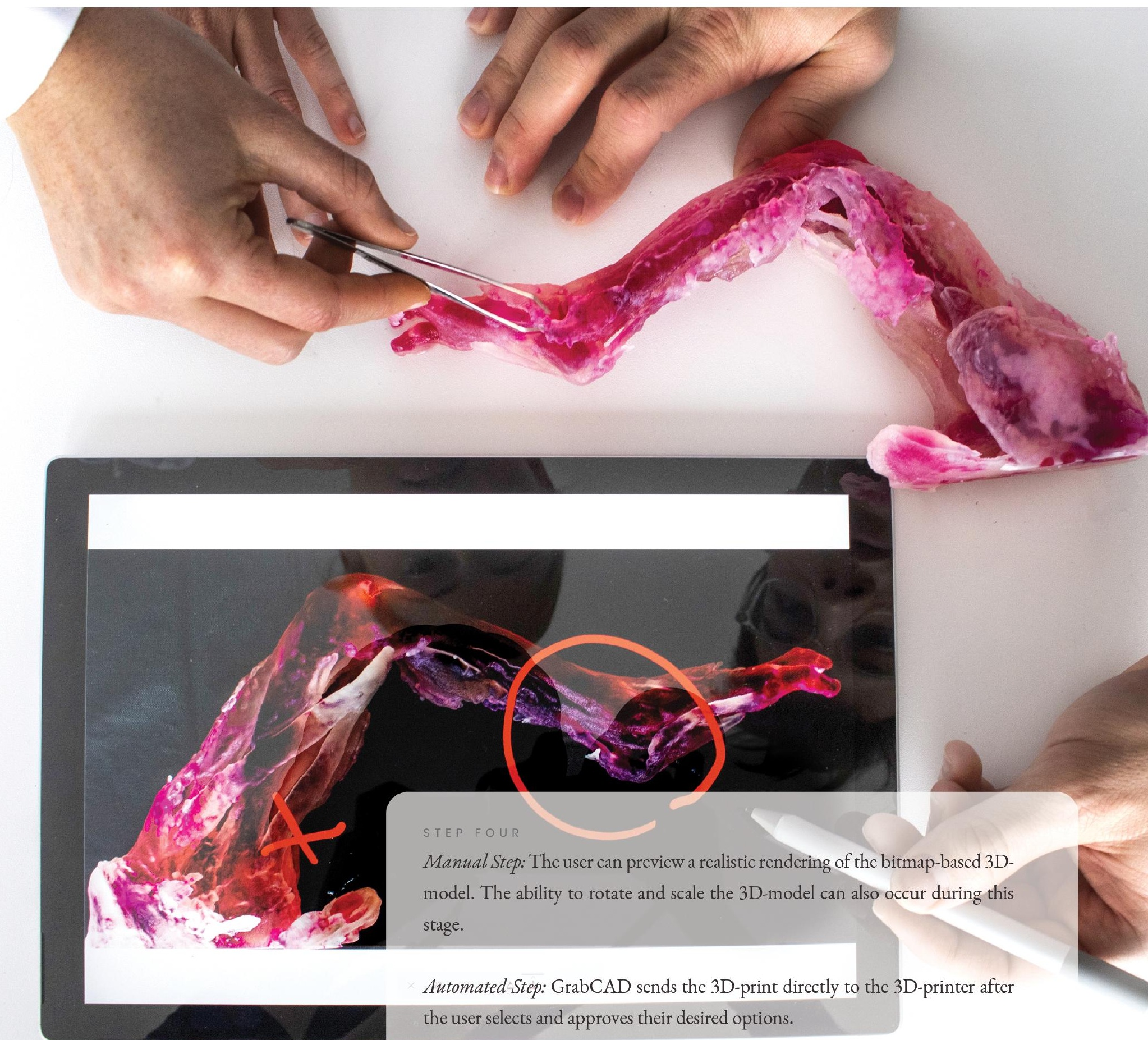

C 
STEP FIVE

Interacting with the model - just like the user planned to!
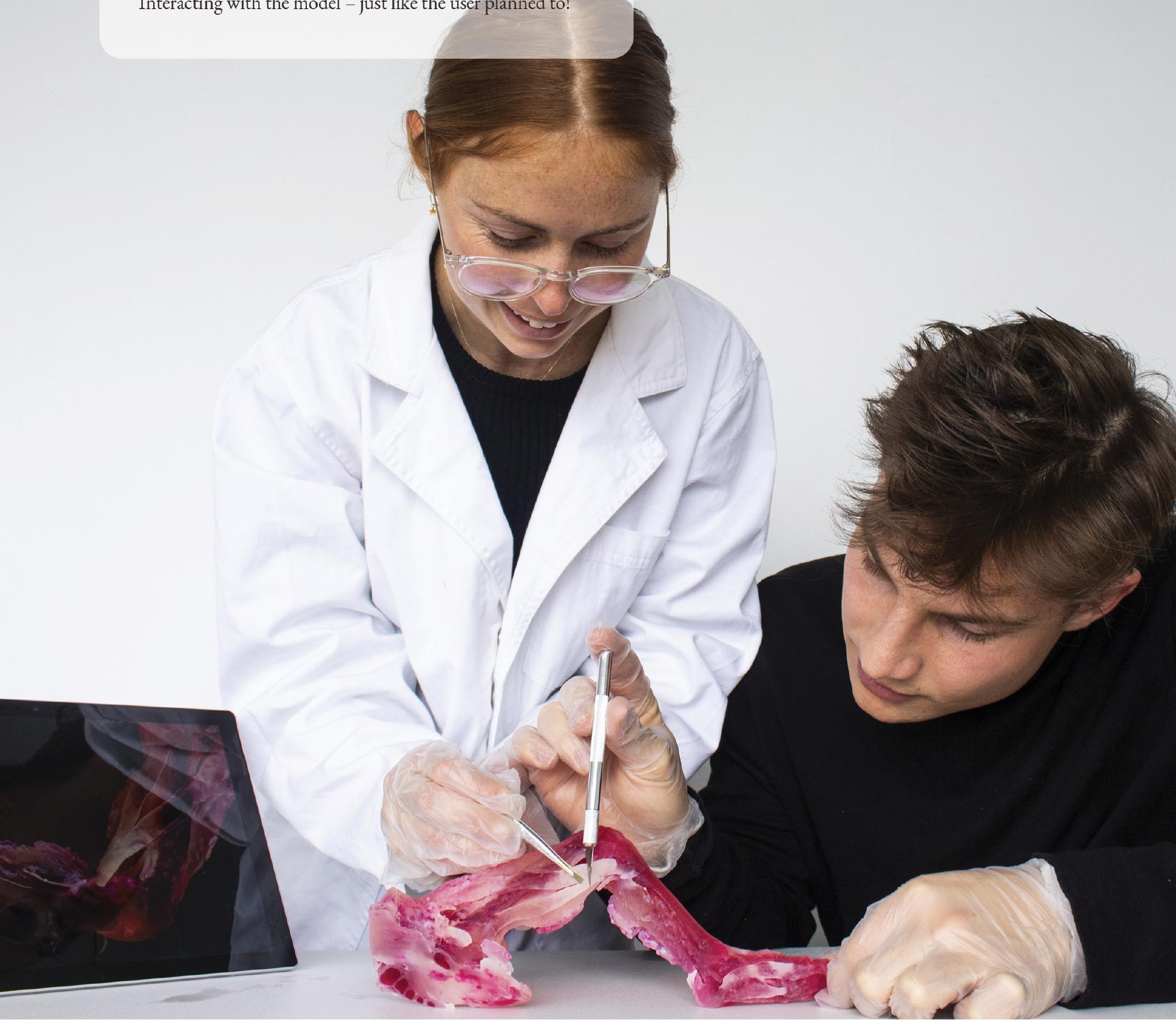

D 



$$
05
$$




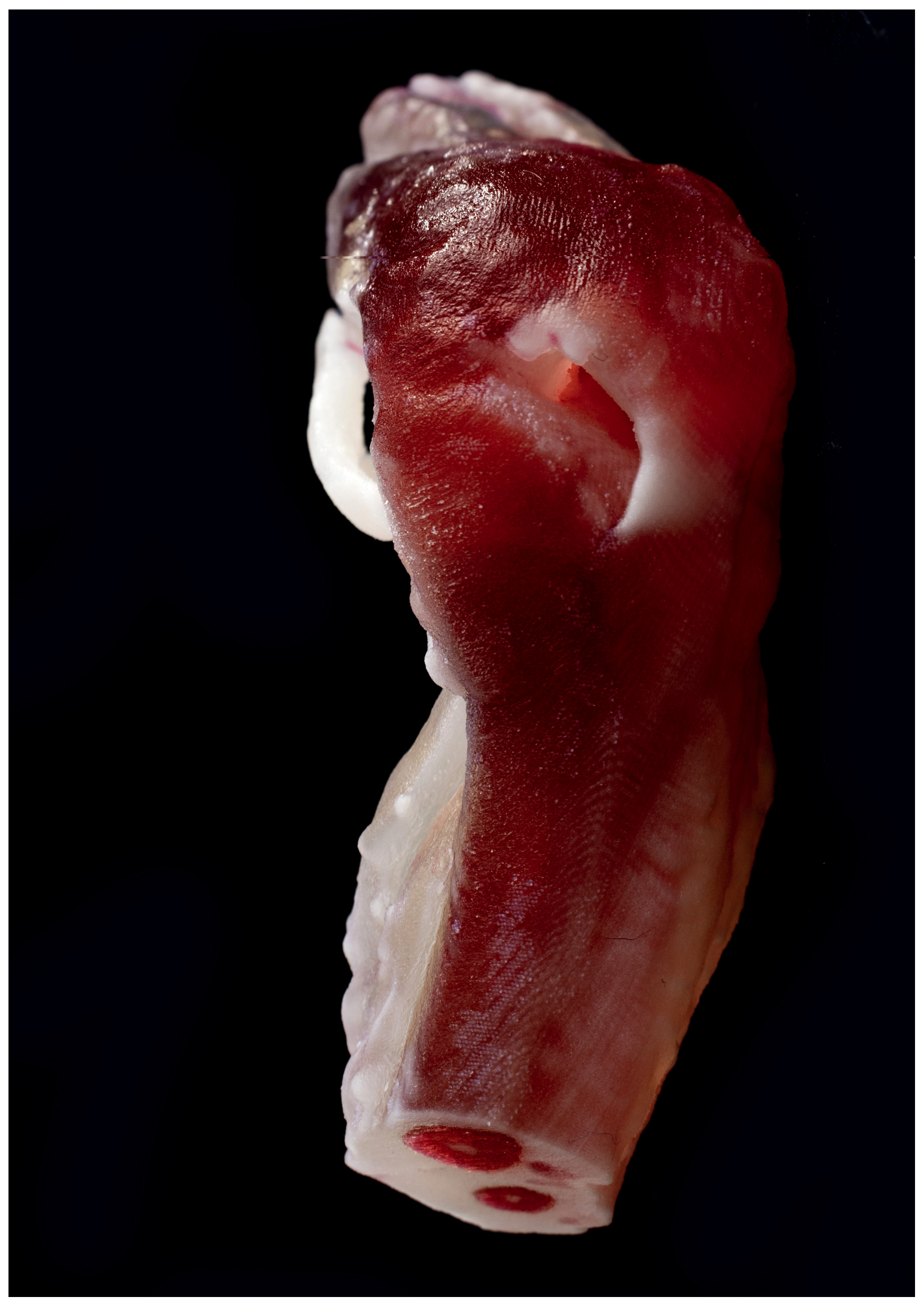

Figure 154. A bitmap-based 3D-printed knee (3D-print \#33). 


\subsection{KEY FIN DINGS}

The purpose of this research was to understand the opportunities of a bitmapbased 3D-printing workflow using the Stratasys $\mathrm{J} 750^{\mathrm{TM}}$ 3D-Printer range for the production of high-fidelity and patient-specific medical models of anatomy. The bitmap-based 3D-printing workflow presented in this research utilised biomedical imaging data to produce 3D-printed models of anatomy, with the ultimate aim of replicating realistic anatomical colour and density.

Three overarching research methodologies (Research into Design, Research through Design and Research as Design) worked in combination to form the research framework. Evolving design criteria were used throughout the research to narrow down the field of exploration, setting clear goals for each Research Stage. The design criteria served as the starting point - and means of assessment - for parallel, refined and final prototyping. The physical design process presented in the Case Studies followed a Practical Action Research Cycle. This involved observing, planning, prototyping, documenting and modifying designs to meet the design criteria.

The developed bitmap-based AM workflow successfully produced patient-specific medical models from different forms of medical data. The results of this research demonstrate the Stratasys J750 ${ }^{\mathrm{TM}}$ 3D-Printer's capability to mimic anatomical colour and density within synthetic 3D-printed anatomical models, highlighting the advantages of bitmap-based 3D-printing for highly realistic and efficient medical modelling workflows.

This research found that the production of highly accurate models - with graduated densities, translucency, colour and flexion - overcame complexities that typically arise through traditional mesh-based medical modelling methods. Notably, the research results suggest that bitmap-based 3D-printing can create models that cannot be produced through any other manufacturing method. Bitmap-based 3D-printing affords the designer the ability to engineer complex material combinations, and control over the material quality and placement of every 14-micron voxel - opening up a world of opportunities in medical design, anatomical visualisation and communication. 


\subsection{NTERPRETATION S}

\section{2.1}

\section{case study 1}

In Case Study 1, anatomical colour was successfully mimicked using bitmapbased 3D-printing and the Visible Female ${ }^{\circledR}$ Dataset (cryosection images). Available literature suggests that the highly realistic replication of anatomical features and colour within a 3D-printed model (such as the 3D-prints presented in Case Study 1) is unprecedented. The results of Case Study 1 demonstrate that realistic anatomical colour can be mimicked in synthetic models of anatomy using a bitmap-based 3D-printing workflow, appearing almost identical to the cryosection images of the cadaveric specimen.

Through iterative parallel prototyping, the most efficient bitmap-based 3Dprinting workflow for the conversion of cryosection images to a 3D-printed model was reached (as outlined in Figure 38.). Comparing the bitmap-based 3D-prints with the full colour photographic images of the Visible Female ${ }^{\circledR}$ served as a method of colour accuracy evaluation.

The relationship between 3D-print \#7 and 3D-print \#8 suggests that VeroClear ${ }^{\mathrm{TM}}$ can significantly alter the appearance of anatomical colour, creating a green and dark finish. This discovery suggests that to generate realistic anatomical colour, full-colour bitmap images should be digitally halftoned using $100 \%$ pixel diffusion ratio (allowing materials to blend smoothly). VeroClear ${ }^{\mathrm{TM}}$ should only be utilised in small quantities or as a clear bounding box, but should not be included within the anatomical area of the 3D-print.

The differences between 3D-prints \#5 and 3D-print \#6 suggest that including thin layers of SUP706 ${ }^{\mathrm{TM}}$ within a 3D-print may cause a collapse in structure, change in colour and the production of a surface texture. In 3D-print \#6 SUP706 ${ }^{\mathrm{TM}}$ was used to replace VeroMagenta-V ${ }^{\mathrm{TM}}$ on every other 27 -micron bitmap layer. This may explain why one side of the 3D-print appears more yellow (due to the decrease in VeroMagenta-V ${ }^{\mathrm{TM}}$ ). In contrast, 3D-print \#5 was produced using the same bitmap image stack as 3D-print \#6, but was 3D-printed without the inclusion of SUP706 ${ }^{\mathrm{TM}}$.
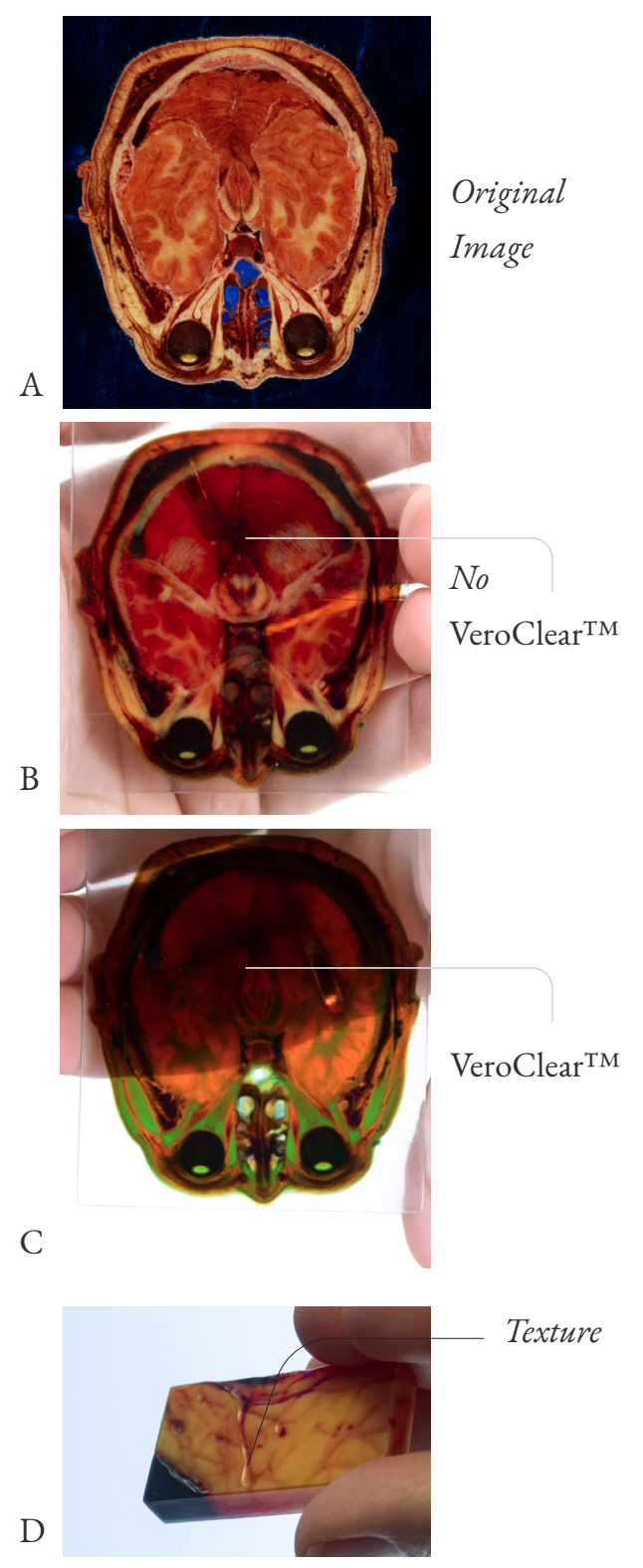
3D-print \#12, which includes the material Agilus30 $30^{\mathrm{TM}}$, was combined with Vero $^{\mathrm{TM}}$ materials with the aim of producing a 3D-print with integrated dynamic movement as well as realistic anatomical colour. However, the resulting 3D-print suggests that when Agilus30 $30^{\mathrm{TM}}$ is dispersed within a 3D-print, the colour fades substantially. Moreover, as soon as Vero ${ }^{\mathrm{TM}}$ is combined with Agilus $30^{\mathrm{TM}}$, the 3D-print hardens. A plausible explanation for this could be that Vero ${ }^{\mathrm{TM}}$ works as a catalyst, and when even a small amount is added to Agilus $30^{\mathrm{TM}}$, the 3D-print drastically hardens. This had significance in relation to the overall study because, when investigating 3D-printed dynamics in Case Study 2, the softest areas of anatomy had to sacrifice colour pigmentation.

Information gathered from parallel prototyping in Case Study 1, meant that a 1:12 full-colour model of the Visible Female ${ }^{\circledR}$ was 3D-printed using the developed bitmap-based 3D-printing workflow. The results of Case Study 1 far outweighed the results that were expected from this section of the research. This is because the colour quality that was mimicked was far more realistic and details were of higher fidelity than initially expected.

Overall, all three criteria outlined for Case Study 1 were successfully met because an efficient bitmap-based 3D-printing workflow was developed, a design experimentation was conducted, and a final model was produced with an unprecedented level of detail.

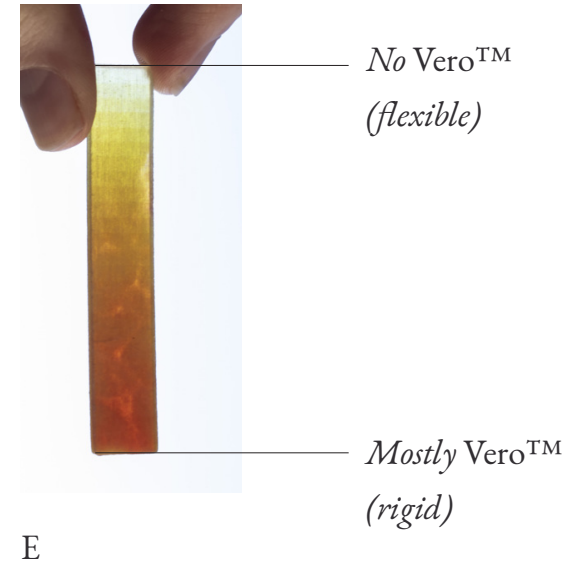

$\mathrm{E}$ 


\section{2.2}

\section{case study 2}

Case Study 2 focussed on mimicking anatomical dynamics by using a bitmapbased 3D-printing workflow and patient-specific biomedical imaging data (CT data). A bitmap-based workflow that turns greyscale CT data into models with graduated densities and colour was successfully produced. However, results of Case Study 2 indicated that 3D-printing materials available on the Stratasys $\mathrm{J} 750^{\mathrm{TM}} 3 \mathrm{D}-$ Printer were not soft enough to mimic anatomical density and biomechanics to the highest degree of realism.

Successfully replicating anatomical density and movement from biomedical imaging data was a more difficult task than mimicking anatomical colour. Factors that influence the degree of 3D-printing difficulty include the resolution of the biomedical imaging data and material elasticity ('softness') - variables that are not always within the control of the designer. Unlike Case Study 1, the patient-specific CT data was greyscale and not full-colour. Accordingly, producing anatomical colour as well as density within a 3D-printed model using greyscale data was a huge challenge.

Case Study 2 presented bitmap-based 3D-printed models of a neonatal leg that included graduated density, translucency and colour after reaching the most suitable bitmap-based medical modelling workflow through initial design experimentation and refined prototyping. Initial design experimentation suggested that 3D-printed movements (dynamics) were most successful when the original greyscale bioimaging data was interpolated at the native 3D-printer $z$ resolution, digitally halftoned using black and white pixels and assigned materials in the post-processing stage (on GrabCAD software). The results of the initial design experiments showed that this workflow preserved the highest amount of anatomical detail within the 3D-print (outlined in Figure 93.).

Before conducting Case Study 2, no previous research that quantified whether bitmap-based 3D-printing using Stratasys J750 ${ }^{\mathrm{TM}}$ 3D-printing materials created synthetic equivalents to human anatomy. Moreover, there were no existing 
bitmap-based 3D-printed dynamic models to use as design precedents. Therefore, the focus and evaluation of Case Study 2 results involved a qualitative analysis - including stop motion photography, tactile observations and the presentation of work to experts in respective design, engineering and medical fields.

Combining Agilus $30^{\mathrm{TM}}$ with $\operatorname{Vero}^{\mathrm{TM}}$ created the most structurally sound models. The results of Case Study 2 suggest that integrating SUP706 ${ }^{\mathrm{TM}}$ within a 3D-print can sacrifice structural integrity. Interestingly, as seen in 3D-print \#24, evenly dispersing SUP706 ${ }^{\mathrm{TM}}$ within Agilus $30^{\mathrm{TM}}$ can create a softer, more flexible material whilst maintaining its structural integrity. Although this combination worked well for 3D-print \#24, this technique can also allow for errors on the building platform - as observed in 3D-prints \#22, \#23, \#25 and \#26. The results might suggest that, because SUP706 ${ }^{\mathrm{TM}}$ is not a material designed for modelling, SUP706 ${ }^{\mathrm{TM}}$ sinks or collapses when 3D-printed as part of a model rather than as the support structure.

When thin layers of Agilus30 $30^{\mathrm{TM}}$ skin are added to a 3D-print, such as 3Dprints \#26 and \#26, the skin risks being 'pierced' and peeling away, exposing the SUP706 ${ }^{\mathrm{TM}}$ material (which can then wash away and distort the model form). This, however, was improved in 3D-print \#36, where the skin was thicker and successfully encased SUP706 ${ }^{\mathrm{TM}}$.

Pixel diffusion ratios within bitmap images aimed to reflect the greyscale H.U. values of the CT scan data. The ratio of soft (Agilus $30^{\mathrm{TM}}$ ) material to hard $\left(\right.$ Vero $\left.^{\mathrm{TM}}\right)$ material determines the approximate pixel greyscale value. 
Digital halftoning algorithms were successfully used to create a 1:1 scale 3Dpritned model of a neonatal leg. The leg was replicated to the highest degree of realism, with dynamics embedded within the 3D-print. The joints, however, did not articulate as hypothesised. Due to the rigid nature of Agilus $30^{\mathrm{TM}}$, the joint 3D models and the full leg model did not articulate realistically when 3D-printed. However, thinner areas of the leg (such as the tendons) which contained less Vero ${ }^{\mathrm{TM}}$ and thinner layers of Agilus $30^{\mathrm{TM}}$, moved with full flexion. 3D-prints \#27, \#28 and \#29 suggest that when Agilus30 $0^{\mathrm{TM}}$ is 3D-printed in thinner layers it is considerably more flexible than when it is 3D-printed in thicker layers such as 3D-prints \#30,\#32, and \#34.

The full leg was 3D-printed using a 30\% diffusion dithering algorithm due to the limited amount of flexion achieved through blending $\mathrm{Vero}^{\mathrm{TM}}$ into Agilus $30^{\mathrm{TM}}$ at a $70 \%$ pixel diffusion ratio presented in 3D-prints \#30, \#32, and \#34. It is important to note that a $0 \%$ diffusion pixel ratio means that no material blending occurs at all (like a mesh-based file) and, therefore, selecting the appropriate diffusion ratio is a critical step in the design process.

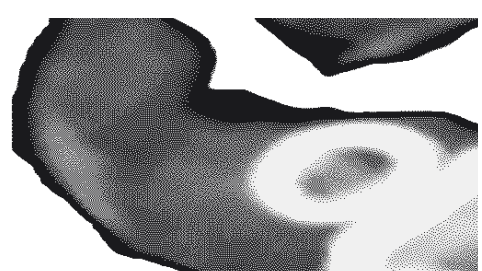

Figure 156. 70\% diffussion

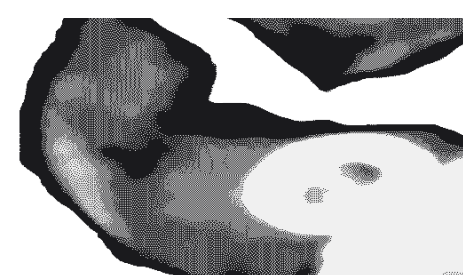

Figure 157. 30\% diffussion

Overall, Case Study 2 helped to develop an effective bitmap-based 3D-printing workflow for the conversion of CT data into dynamic bitmap-based 3D-printed models. This workflow translated the CT data into 3D-printed models with gradients in density and colour, ranging from very soft 3D-printed material (mimicking fat) to harder areas of anatomy such as bone. The final design, a 1:1 scale model of a neonatal leg, was unprecedented in its fidelity and workflow (blending both the density and colour of materials). However, the inherent rigidity of Stratasys $\mathrm{J} 750^{\mathrm{TM}}$ 3D-printing materials meant that joint articulation was not achieved to its desired potential and therefore did not mimic the biomechanics of human anatomy to the highest degree of realism. 


\section{2.3}

\section{Case study 3}

In response to limitations identified in Case Study 2, Case Study 3 focussed on testing novel Stratasys J750 TM Digital Anatomy ${ }^{\mathrm{TM}}$ 3D-printing materials. The new Digital Anatomy ${ }^{\mathrm{TM}}$ 3D-printing materials were developed by medical professionals, along with engineers at Stratasys, and have undergone rigorous assessment in terms of their likeness to human anatomy. The results of Case Study 3 suggest that the Stratasys J750 ${ }^{\mathrm{TM}}$ Digital Anatomy ${ }^{\mathrm{TM}}$ 3D-printing materials are substantially softer than the Stratasys $\mathrm{J} 750^{\mathrm{TM}} 3 \mathrm{D}$-printing materials. The elasticity of the newly developed TissueMatrix ${ }^{\mathrm{TM}}$ material meant that the $3 \mathrm{D}$ prints could move, bend, stretch and return to their original state, mimicking human anatomy to a higher degree of realism than materials used in Case Study 1 and 2 .

Purely based on qualitative tactile observation, Digital Anatomy ${ }^{\mathrm{TM}}$ 3D-printing materials were compared to the Stratasys $\mathrm{J} 750^{\mathrm{TM}}$ 3D-printing materials. The Stratasys J750 ${ }^{\mathrm{TM}}$ Digital Anatomy ${ }^{\mathrm{TM}}$ 3D-Printer has six native 3D-printing materials including Tissue Matrix ${ }^{\mathrm{TM}}$, BoneMatrix ${ }^{\mathrm{TM}}$, GelMatrix ${ }^{\mathrm{TM}}$, Agilus $30^{\mathrm{TM}}$ and two Vero ${ }^{\mathrm{TM}}$ materials. GrabCAD Print allows a designer to assign each mesh with over 100 different Digital Anatomy ${ }^{\mathrm{TM}}$ materials. This suggests that Stratasys J750 ${ }^{\text {TM }}$ Digital Anatomy ${ }^{\mathrm{TM}}$ 3D-printing materials on GrabCAD are comprised of voxel material combinations - ratios between the six native 3D-printer materials. It is hypothesised that the voxel ratios and diffusion dithering algorithms determine the density and microstructures within each Digital Anatomy $^{\mathrm{TM}}$ material available for 3D-printing.

Case Study 3 concluded by creating a design concept for bitmap-based 3Dprinting medical modelling software that is primarily automated with some user control.

Overall, Case Study 3 explored new 3D-printing materials on the Stratasys J750 ${ }^{\mathrm{TM}}$ Digital Anatomy ${ }^{\mathrm{TM}}$ 3D-Printer and concluded, based on tactile analysis, that Digital Anatomy ${ }^{\mathrm{TM}}$ materials are substantially more 'human-like' than Stratasys $\mathrm{J} 750^{\mathrm{TM}} 3 \mathrm{D}$-printing materials. 


\subsection{M PLICATIONS}

The results presented in this research portfolio have built on a small number of bitmap-based 3D-printing studies presented in published literature (Bader et al., 2016, 2018; Brunton et al., 2015; Hosny et al., 2018; Kolb, 2017; Stute et al., 2018), adding to the existing body of evidence that bitmap-based 3D-printing can create models with unprecedented material qualities due to the production of material gradients that can be engineered at a 14-micron voxel resolution. Previous research has had a strong emphasis on bitmap-based 3D-printing to produce Vero ${ }^{\mathrm{TM}}$ (rigid) models that do not have any form of flexion or dynamic movement. In comparison, this research commenced by producing 3D models using solely Vero ${ }^{\mathrm{TM}}$, but then progressed to integrating both soft (Agilus30 ${ }^{\mathrm{TM}}$ ) and hard $\left(\right.$ Vero $^{\mathrm{TM}}$ ) materials into one 3D-print - an area of research that has been relatively unexplored.

The results of this research portfolio provide new insights into the relationship between Agilus $30^{\mathrm{TM}}$ and Vero ${ }^{\mathrm{TM}}$ materials, including how Agilus $30^{\mathrm{TM}}$ and Vero $^{\mathrm{TM}}$ interact in bitmap-based 3D-prints. All three Case Studies tackled relatively unexplored areas of research and provided a clearer insight into mimicking anatomy using patient-specific scans, bitmap-based 3D-printing workflows, and novel medical modelling materials. 
5. 3.1

Bit map-Based Vs. Mes h-Based 3 D-Printing

Case Study 2 and 3 both 3D-printed neonatal feet from the same CT dataset. Case Study 2 used a bitmap-based 3D-printing workflow and Case Study 3 used a traditional mesh-based 3D-printing workflow. Comparing the resulting 3Dprints from both Case Studies provides new insight into the advantages and disadvantages of both medical modelling workflows.

This research suggests that a bitmap-based 3D-printing workflow can produce patient-specific medical models in significantly less time and at a higher fidelity than traditional medical models.

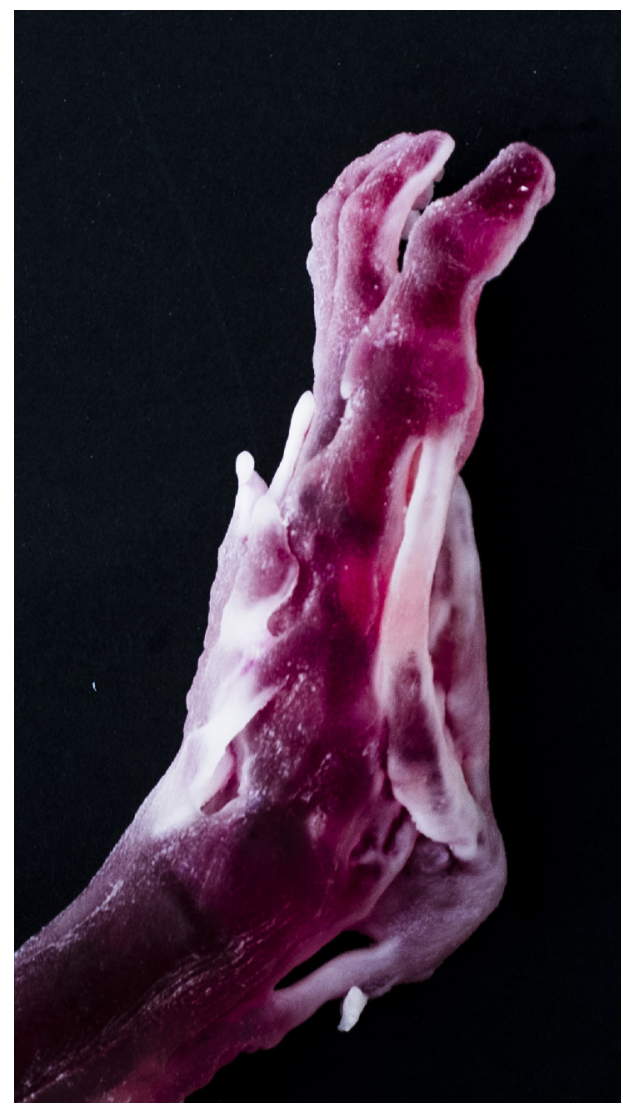

Case Study 2(bitmap-based) 3D-print

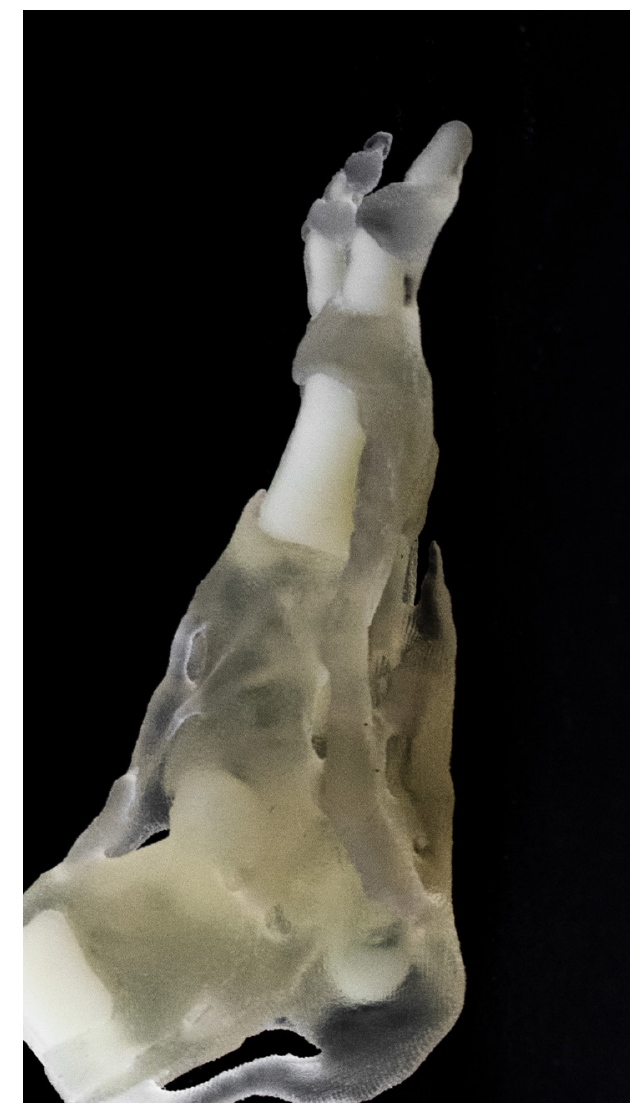

Case Study 3 (mesh-based) 3D-print

Figure 158. A comparison between a bitmap-based (3D-print \#31) and a mesh-based (3D-print \#49) 3Dprinted neonatal foot. 
The results of this research build on the existing body of evidence that suggests that traditional mesh-based medical modelling workflows are time consuming, resulting in anatomically inaccurate 3D-prints caused by human error, data loss and multiple post-processing steps (Bader et al., 2018, 2018; Hosny et al., 2018; Huotilainen et al., 2014). The research results suggest that bitmap-based 3D-printing files for medical modelling (GCVF files) require a significantly less time to generate than complex mesh-based files (STL files) due to the automated nature of the workflow and the synonymity between medical bioimaging datasets and bitmap-based 3D-printing files.

Bitmap-based 3D-printing not only bypasses thresholding steps used in traditional segmentation methods, it also reduces the amount of computer processing power needed to create multimaterial 3D-prints. Traditional medical modelling workflows include a processing step, which requires the designer to perform what is known as a "Boolean" or "Cut out" of every anatomical "nestled" anatomical feature (no meshes can intersect). Because shape specification is inherently linked to one colour and density, each mesh needs to be separate from another if they require a different material. This "Boolean" step is timeconsuming and entails a huge amount of computer processing power (especially if the model is complex and requires a range of different materials).

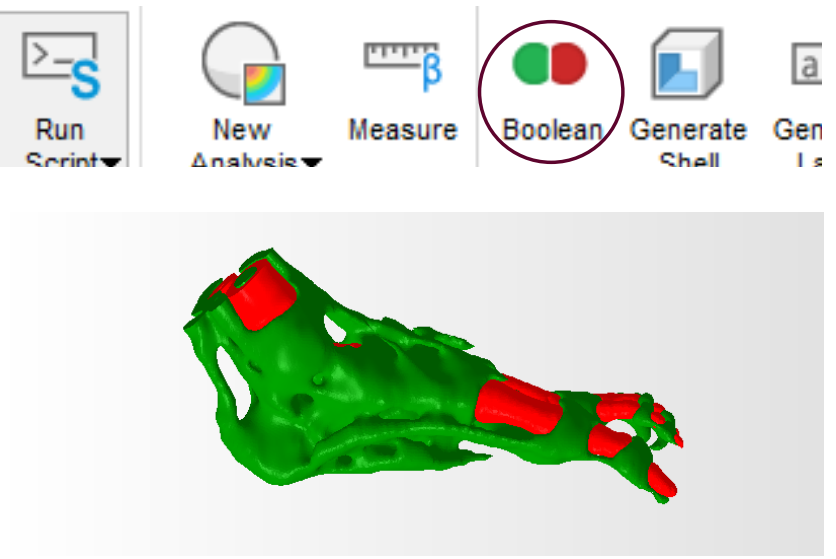

Figure 159. Boolean function.
In Case Study 3, the mesh-based files were difficult to produce and prepare for multi-material 3D-printing in comparison to bitmap-based 3D-printed files presented in Case Study 2 (even though the mesh-based 3D-prints only included three different anatomical STL meshes). The automatic nature of a bitmap-based 3D-printing workflow eliminates the need for manual file processing including the need to "Boolean" mesh-files. This study therefore challenges the efficiency of traditional segmentation techniques and emphasises that current production methods completely overlook the production of heterogenous material distributions created through bitmap-based 3D-printing (Bader et al., 2016).

A recent economic analysis of the cost-saving potential of 3D-printed anatomical models for surgical applications shows that 3D-printed anatomical models drastically decrease operating room costs secondary to shortening procedure times (Ballard et al., 2020, p. 1103). The use of 3Dprinted medical models resulted in a mean reduction time of 62 minutes and USD $\$ 3,720$ per operation due to shorter and more efficient procedures (Ballard et al., 2020). Because preparing bitmap-based files for 3D-printing required far less processing time, there is considerable potential for bitmapbased 3D-printing to save additional costs associated with labour intensive and mesh-based medical modelling. Bypassing the traditional segmentation conversion process streamlines the medical modelling workflow, decreasing time and, subsequently, the costs associated with model production. 


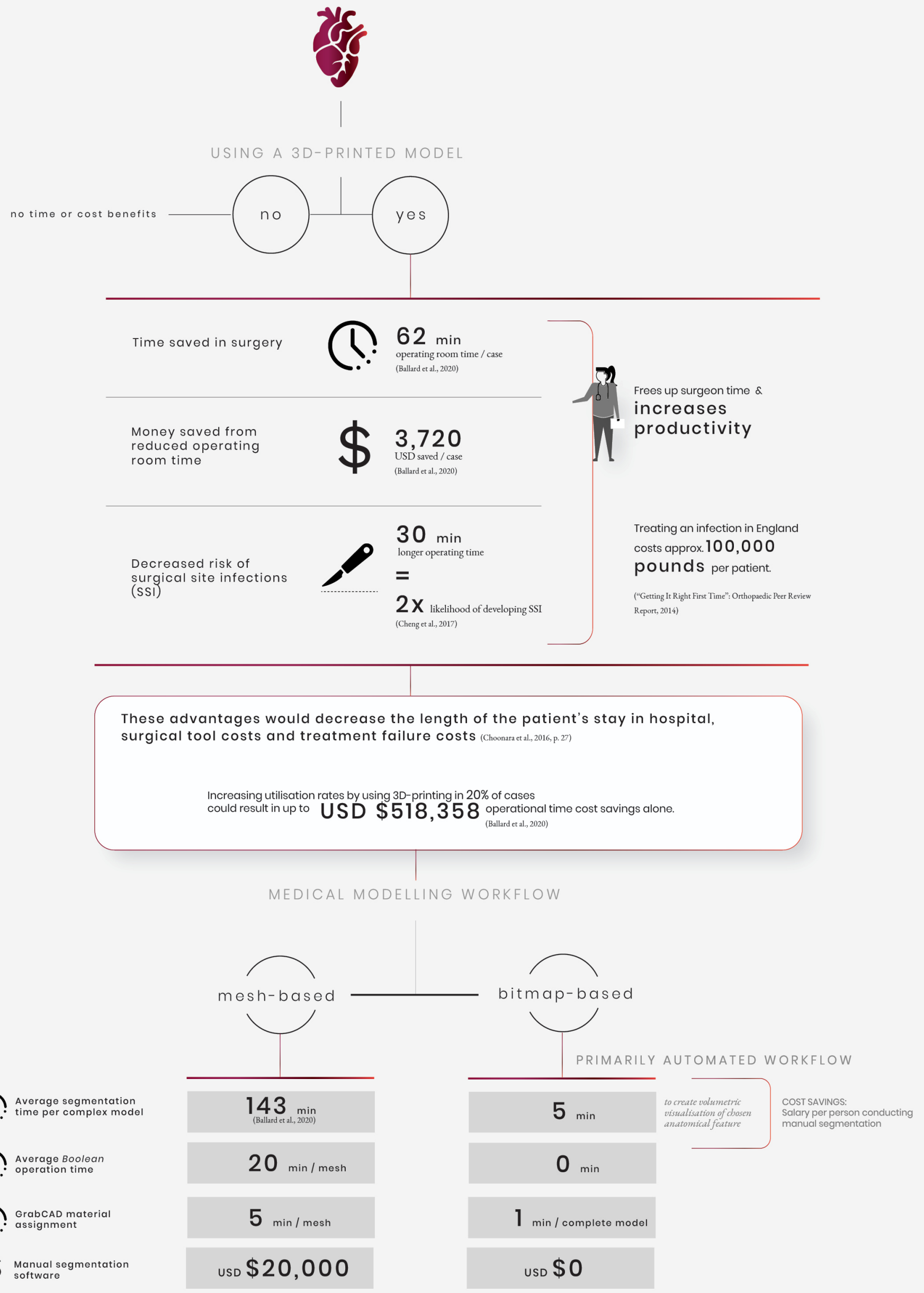

Figure 160. Time and cost savings of bitmap-based 3D-printed models. 
The level of detail achieved through bitmap-based 3D-printing for the production of medical models with gradated density and colour is superior to any form of mesh-based 3D-printing. This is evident in the 3D-print of the Visible Female ${ }^{\circledR}$ presented in Case Study 1. Blending colours at a high-fidelity using a bitmap-based 3D-printing workflow would be impossible to achieve through any other mesh-based 3D-printing workflow. Like 2D-printing, bitmap-based 3D-printing workflows can blend colours to produce photo realistic 3D-printed models. A previous study texture mapped realistic anatomical colour to surface voxels around a white $3 \mathrm{D}$-printed core, rather than throughout an entire 3D-printed model (Brunton et al., 2015). The results presented in Case Study 1 challenge the concept of needing a white core embedded within a 3D-print to produce photorealistic colour. Instead, removing VeroClear ${ }^{\mathrm{TM}}$ and integrating an appropriate amount of VeroPureWhite ${ }^{\mathrm{TM}}$ can create more realistic colour within a $3 \mathrm{D}$-print.

The differences in detail between the traditionally segmented mesh-based and bitmap-based 3D-printed neonatal feet highlight some of the advantages of bitmap-based 3D-pritnting workflows using biomedical imaging data. By comparing files produced in Case Study 2 and 3 to create 3D-printed neonatal feet, the amount of data integrated within to the bitmap-based 3D-print in Case Study 2 is far greater than the integrated data in the mesh-based 3D-print produced in Case Study 2.

Each 3D-printed neonatal foot (3D-print \#30 and \#31) created in Case Study 2 was produced by sending 1, 494 cross-sectional bitmap images to the Stratasys J750 TM 3D-Printer. Each of those cross-sectional bitmap images was comprised of a pixel matrix containing $1,483,524$ pixels $(1,483,5243 \mathrm{D}$-printed voxels). This means that each bitmap-based 3D-printed foot presented in Case Study 2 was constructed from 2,216,384,856 voxels (1,483,524 pixels x 1,494 images). Material qualities and details produced by having control over every one of the 2,216,384,856 14-micron voxels constructing the 3D-printed neonatal foot far surpasses the potential detail achieved through mesh-based 3D-printing workflows. In comparison, the same neonatal foot presented in Case Study 3 (3D-print \#49) was created form two individual surface meshes (less detail) but involved a longer processing time.

Control over every voxel also presents other advantages over mesh-based 3D-printing workflows. Anatomical features using bitmap-based 3D-printing files are not restricted to one material specification - meaning that the 3D-prints can contain gradients in both density and colour, mimicking human anatomy to a higher degree of realism. 


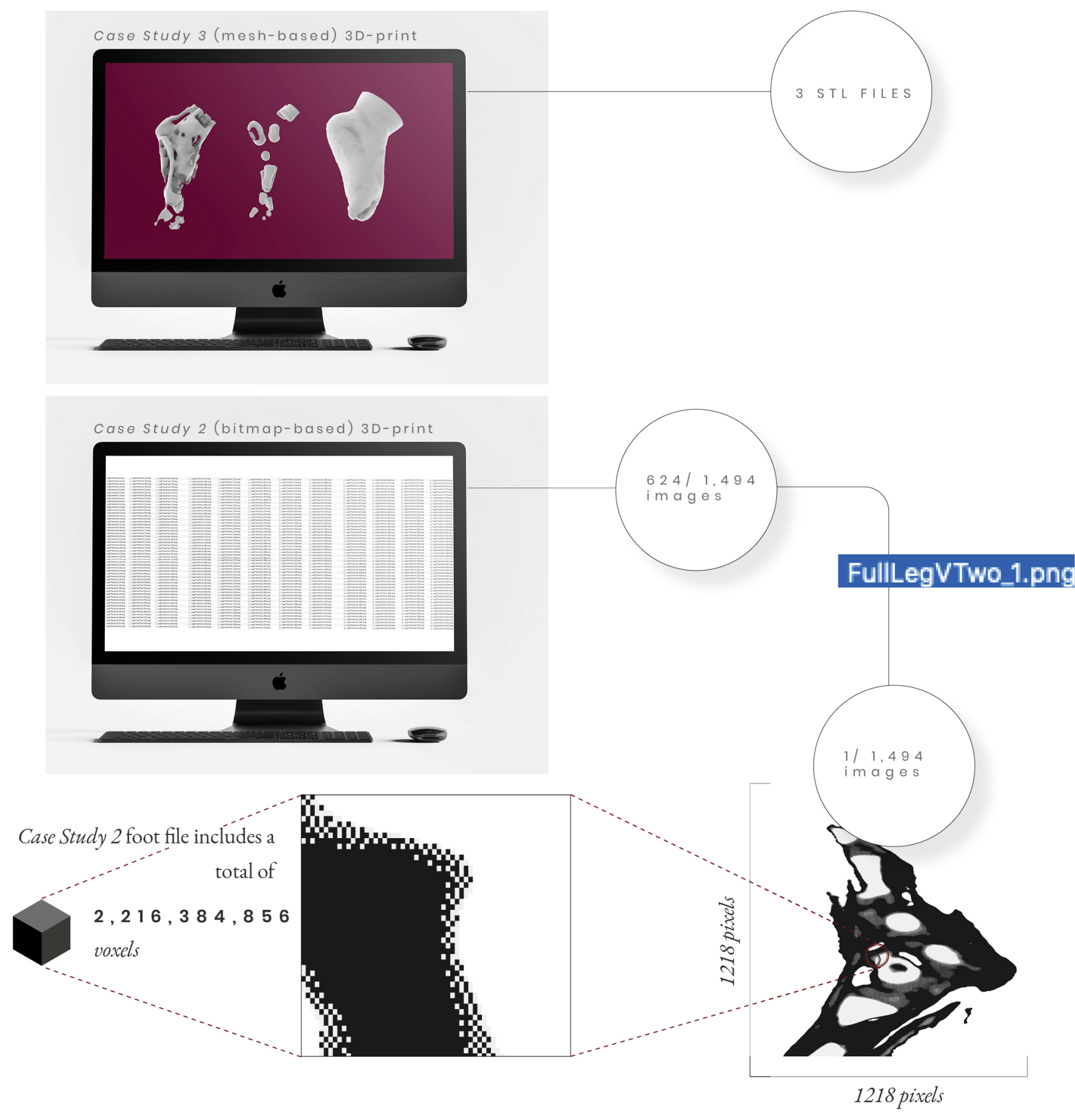

Figure 161. Bitmap-based 3D-printing vs. mesh-based 3D-printing (detail). 


\section{3 .2}

\section{D-Printing Theory and Practice}

THE ROLE OF THE PHYSICIAN AND THE DESIGNER

This research should be considered when addressing the future of 3D-printing theory and practice. Medical modelling using novel technology and a bitmapbased 3D-printing workflow is inherently an inter-disciplinary area of research requiring a range of different skill sets. From medical clinicians to creative design thinkers - a variety of strengths are required to develop bitmap-based medical modelling. A common language of communication and adaptable skills are required to propel this area of research forward. This, in turn, presents a new way of thinking and considerable potential for new professions that lie in the intersection of design and medicine.

AUTOMATION IN MEDICINE

The application of artificial intelligence (AI) systems has grown drastically in virtually every discipline including medicine, design and data processing (Ferretti et al., 2018, p. 320). AI systems with integrated machine learning have the ability to learn and complete tasks on their own or guided slightly by human supervision (Ferretti et al., 2018, p. 320). An increase in research is currently being conducted into the applications and validity of automated and semi-automated segmentation techniques for medical modelling (Rizwan I Haque \& Neubert, 2020; Sharma et al., 2010). The value of automated segmentation techniques lies in its ability to achieve fast and accurate results which are not influenced by influences such as fatigue or human error in manual segmentations steps (Sharma et al., 2010). Results presented in Case Studies 1 and 2 show that highfidelity medical models can be produced through automated machine learning using a bitmap-based 3D-printing workflow. In turn, decreasing manual labour time and costs. Results presented in Case Studies 1 and 2 contribute to a clearer understanding of the future direction on machine learning for the efficient production of high-fidelity medical modelling. 


\section{3 .2}

\section{CuItura I Implications}

Under the Code of Health and Disability Services Consumers' Rights (HDC Code 1996), every patient in New Zealand has the right to be fully informed (Right 6), along with the right to make an informed choice, and to give informed consent (Right 7). These rights, however, rely heavily on a patient's ability to balance facts, accessible information, and provide an uninfluenced acceptance of treatment. Physical patient-specific models have considerable potential to cross cultural and language barriers, as well as helping patients with deficiencies in hearing and sight. It is, therefore, important to consider the cultural and social impacts of patient-specific medical models for informed consent practices and medical communication.

In te ao Māori, for example, the hauora model of wellbeing encompasses the physical (Taha tinana), mental and emotional (Taha hinengaro), social (Taha whānau) and spiritual (Taha wairua) dimensions of health (Well-being, hauora, n.d.). Decisions regarding an individual's health with a collective cultural framework means important medical decisions are taken by a wider group, not just the individual (Kerse \& Dyall, 2013). Acknowledging and respecting cultural aspects of health have shown to increase overall wellbeing in patients (Kerse \& Dyall, 2013). Taking a patient-specific medical model home and engaging with a patient's community may aid collective decision making, creating cultural and social engagement by democratising important information about health that cannot always be interpreted through $2 \mathrm{D}$ visualisations or verbal communication. 


\subsection{I M I T A T IO N S}

S OFT W ARE

Case Studies 1 and 2 build on the existing evidence that $\mathrm{CAD}$ tools are currently inadequate for bitmap-based 3Dprinting workflows. Successful bitmap-based 3D-printing is based on the designer's ability to explore different software tools and processes, developing their own workflows (Stute et al., 2018). A substantial limitation of bitmap-based $3 \mathrm{D}$-printing workflows is that there is no universal and developed bitmap-based 3D-printing file creation software. Therefore, some design choices were constrained by the lack of developed software for bitmap-based 3D-printing workflows. For example, there is no bitmap-based 3D-printing preview function, which means that bitmap-based 3Dprinted models cannot be previewed (like mesh-based models) before 3D-printing, compromising colour accuracy.

MATERIALS

A limitation of this research was the durability and physical limitations of the Stratasys J750 ${ }^{\mathrm{TM}} 3 \mathrm{D}$-printing material range. Agilus $30^{\mathrm{TM}}$ was not soft enough to mimic soft tissue to the highest degree of realism and therefore articulating joints in the 1:1 neonatal leg was not achieved. Agilus30 $30^{\mathrm{TM}}$ can split at different parts of the models (due to its lack of elasticity), creating breaks and microtears in the material. Polyjet 3D-printing materials are currently plastic and cannot be recycled or reused. Therefore, the sustainability and elasticity of materials is a considerable research limitation.
COVID- 19

COVID-19 and the subsequent Victoria University of Wellington campus closure affected the progression of on-site research using the Stratasys J750 TM 3D-Printer. The impact of the pandemic also meant that conducting additional testing on the Stratasys J750 ${ }^{\mathrm{TM}}$ Digital Anatomy ${ }^{\mathrm{TM}} 3 \mathrm{D}-$ Printer based in China (including bitmap-based 3D-printing using Digital Anatomy ${ }^{\mathrm{TM}}$ ) could not occur.

EVALUATION OF RESULTS

It is beyond the scope of this research to quantify the value and results of the research in a clinical setting. Material qualities of the 3D-printed models need clinical evaluation to quantify their likeness to human anatomy and implementation value.

DIGITAL ANATOMY 3 D-PRINTER

A traditional medical modelling workflow was used in Case Study 3 because the currently available version of GrabCAD for the Stratasys J750 ${ }^{\mathrm{TM}}$ Digital Anatomy ${ }^{\mathrm{TM}}$ 3D-Printer does not have a GrabCAD Voxel Print Utility add-on. 


\subsection{RECOM MENDATIONS}

BITMAP-BASED 3 D-PRINTING ON THE DAP

The capabilities of bitmap-based 3D-printing using the GrabCAD Voxel Print Utility can produced models with an unprecedented level of detail. Further research could test bitmap-based 3D-printing on the Stratasys J750 ${ }^{\mathrm{TM}}$ Digital Anatomy $^{\mathrm{TM}}$ 3D-Printer, blending voxels using the novel and highly realistic medical modelling materials including GelMatrix $^{\mathrm{TM}}$, TissueMatrix ${ }^{\mathrm{TM}}$ and BoneMatrix ${ }^{\mathrm{TM}}$. This has considerable potential to produce the most realistic medical models to date by combining the most successful elements of Case Studies 1, 2 and 3 (workflow of Case Study 2 and materials from Case Study 3).

DEVELOPING BITMAP-BASED 3 D-PRINTING SOFTWARE

Future research could focus on developing a software for the efficient production of bitmap-based 3D-prints. This could mitigate frustrating factors regarding the limited available software tools that are currently used to develop bitmapbased 3D-printing files. The envisioned software would include an automatic 3D-printer $\mathrm{z}$ resolution interpolation function, automatic colour mixing and digital halftoning, a bitmap-based 3D-print colour and geometry preview. It is important to acknowledge how future software could develop a medical modelling process by streamlining the process of converting biomedical bioimaging data into highly realistic bitmap-based 3D-printed models. This could occur by incorporating semi-automated segmentation and the accurate conversion of CT H.U. values to dynamic 3D-printed material combinations that mimic anatomy to the highest degree of realism.
DEVELOPING 3 D-PRINTED MATERIALS

Future studies should take into account that the development of pigmented soft materials (like TissueMatrix ${ }^{\mathrm{TM}}$ ) in Cyan, Magenta, Yellow, Black, White and Clear could produce models that incorporate the visual realism of Case Study 1, the workflow used in Case Study 2 and the tactile realism of materials used in Case Study 3. Future 3D-printing material studies should also take into account the sustainability of materials and potential ways to repurpose and recycle medical modelling Polyjet materials.

QUANTIFYING THE RESEARCH VALUE

A high-fidelity bitmap-based 3D-printing medical modelling workflow offers exciting opportunities for medical education, informed consent practices, and surgical simulation for skills acquisition and pre-operative planning. Technical factors such as the accuracy of a bitmap-based 3D-printing medical modelling workflow, the tactile realism of 3D-printing materials, and the differences between mesh-based and bitmap-based 3D-printing workflows should be evaluated through clinical studies. The resulting quantifying evidence would justify the contextual value of bitmap-based medical modelling for different healthcare applications. 


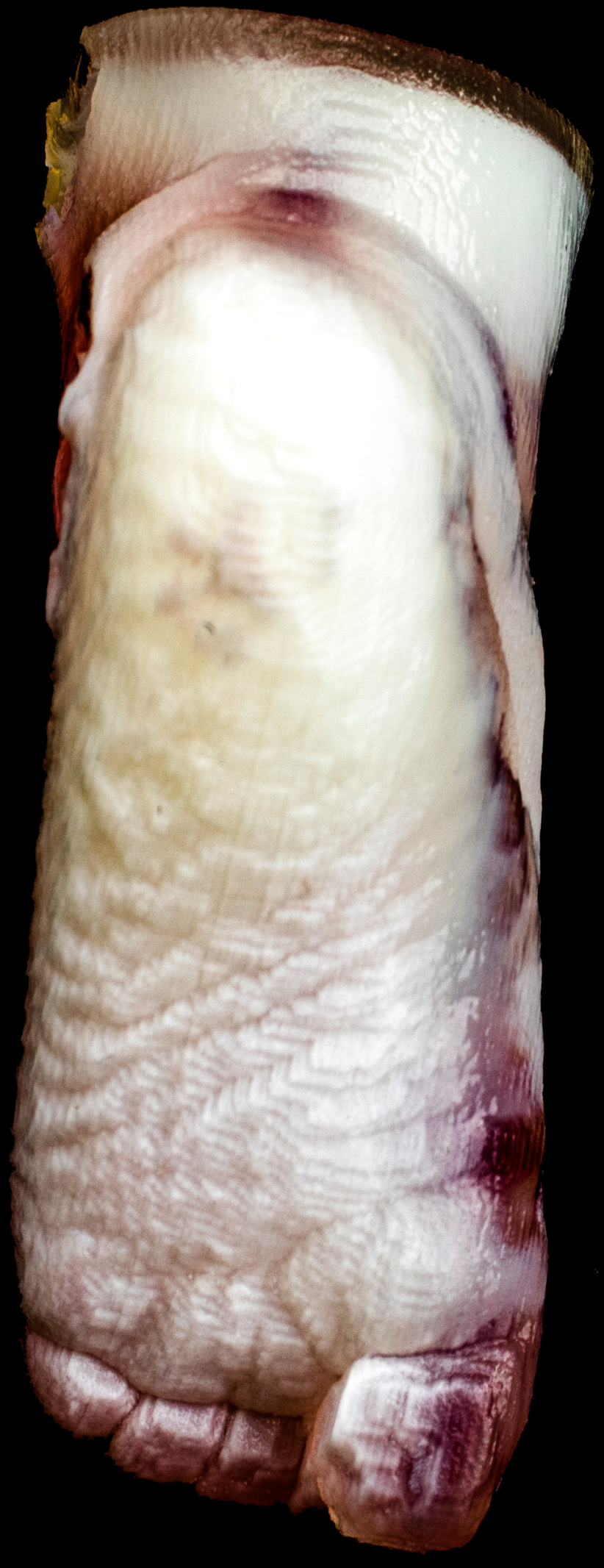




$$
06
$$




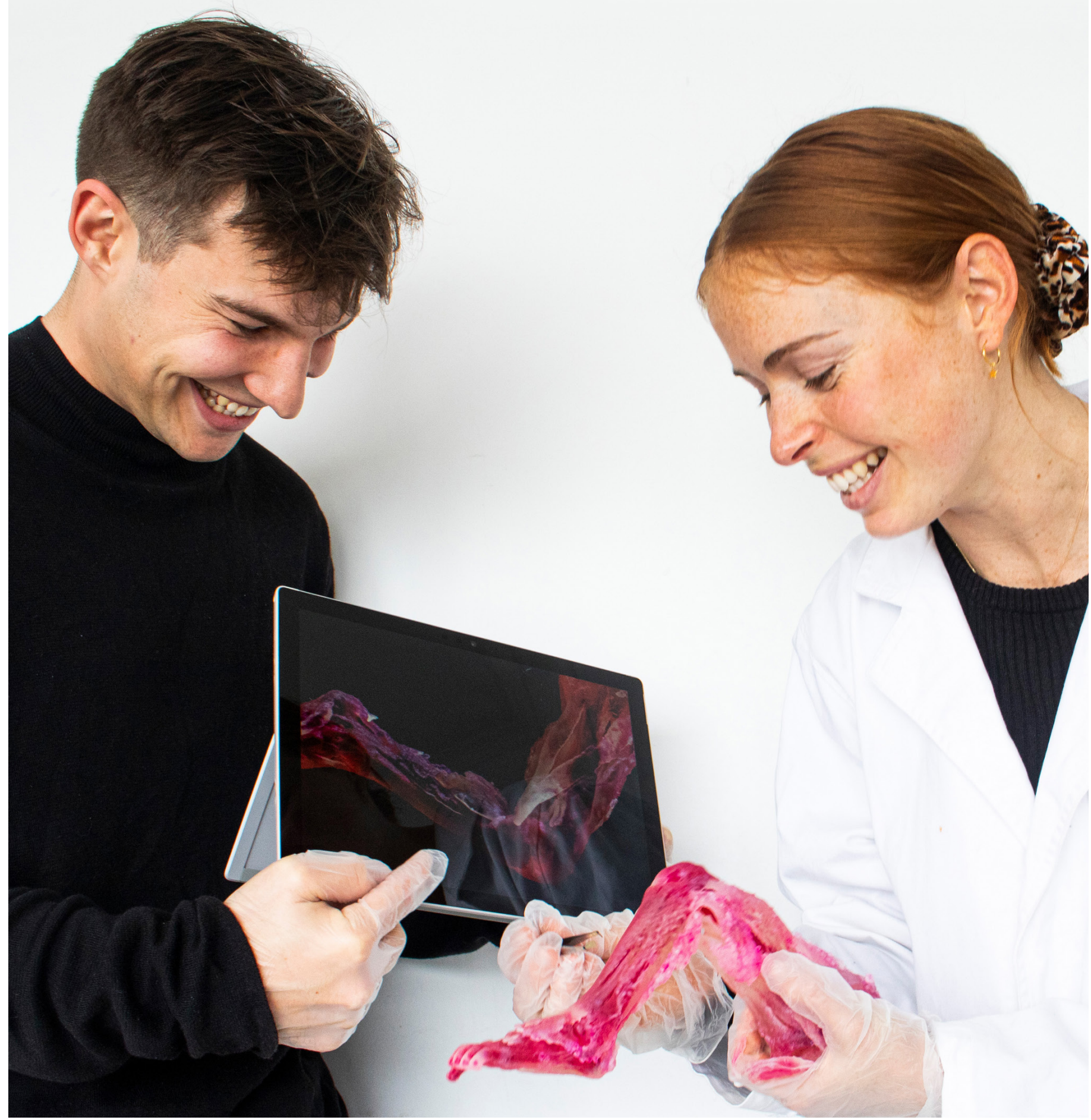

Figure 163. Medical students (models) learning anatomy using a bitmap-based 3D-printed model and a virtual reconstruction of the anatomical part. 


\section{$6.1 \mathrm{CONCLUSION}$}

This research analysed the results of a creative design exploration presented in the form of three different Case Studies. It highlighted the opportunities offered by $3 \mathrm{D}$-printing patient-specific and high-fidelity medical models on multimaterial 3D-printers, with a focus on developing a bitmap-based 3D-printing workflow using biomedical imaging data.

To better understand and develop the presented bitmap-based modelling workflow, materials and results, future studies could address how voxel-based 3D-printed medical models could be developed using Stratasys J750 ${ }^{\mathrm{TM}}$ Digital Anatomy ${ }^{\mathrm{TM}} 3 \mathrm{D}$-printing materials. Further studies may quantify the value of bitmap-based 3D-printed medical models for different contextual applications, such as surgical simulation, pre-operative planning and skills acquisition. By challenging traditional medical modelling workflows, this research addressed the lacuna in existing knowledge. It successfully produced bitmap-based 3Dprints that - with graduated densities, translucency, colour and flexion - overcame complexities that arose due to opaqueness of the human body. A bitmapbased medical modelling workflow has considerable potential to create more detailed models at a faster rate than traditional mesh-based segmentation workflows. Ultimately, this research challenged the way that physical objects are created and has the potential to revolutionise 3D-printing as a whole.

Design is the mover, agent and catalyst for change. This research has demonstrated how design can explore novel technology and translate, in an approachable manner, its potential to society. The presented research highlights the role of the designer and the importance of creativity to challenge, change and advance new manufacturing possibilities. Hopefully, it will encourage collaboration between designers and medical professionals to explore new technologies. Drawing from expertise from across different areas of speciality may advance the quality of medical modelling, with a considerable potential to improve healthcare, save lives and democratise important information about technology and anatomy. In turn, making medical information about anatomy understandable for everyone. 


\subsection{REFERENCES}

Allenby, M. C., \& Woodruff, M. A. (2019). Biofabrication of personalised anatomical models and tools for the clinic. Journal of Cystic Fibrosis, 18(2), 161-162. https://doi.org/10.1016/j.jcf.2019.02.005

Aurell, Y., Johansson, A., Hansson, G., Wallander, H., \& Jonsson, K. (2002). Ultrasound anatomy in the normal neonatal and infant foot: An anatomic introduction to ultrasound assessment of foot deformities. European Radiology, 12(9), 2306-2312. https://doi.org/10.1007/s00330-001-1243-8

Aziz, M., \& Mckenzie, J. (1999). The dead can still teach the living: the status of cadaver-based anatomy in the age of electronic media. Perspectives in Biology and Medicine, 42(3), 402-421. https://doi.org/10.1353/pbm.1999.0014

Bader, C., Kolb, D., Weaver, J., \& Oxman, N. (2016). Data-driven material modeling with functional advection for 3d printing of materially heterogeneous objects. 3D Printing and Additive Manufacturing, 3(2), 71-79. https://doi. $\operatorname{org} / 10.1089 / 3 \mathrm{dp} .2016 .0026$

Bader, C., Kolb, D., Weaver, J., Sharma, S., Hosny, A., Costa, J., \& Oxman, N. (2018). Making data matter: Voxel printing for the digital fabrication of data across scales and domains. Science Advances, 4(5), 1-12. https://doi.org/10.1126/ sciadv.aas 8652

Ballard, D. H., Mills, P., Duszak, R., Weisman, J. A., Rybicki, F. J., \& Woodard, P. K. (2020). Medical 3D Printing Cost-Savings in Orthopedic and Maxillofacial Surgery: Cost Analysis of Operating Room Time Saved with 3D Printed Anatomic Models and Surgical Guides. Academic Radiology, 27(8), 1103-1113. https://doi.org/10.1016/j. acra.2019.08.011

Barber, S. R., Jain, S., Son, Y.-J., \& Chang, E. H. (2018). Virtual functional endoscopic sinus surgery simulation with 3d-printed models for mixed-reality nasal endoscopy. Otolaryngology-Head and Neck Surgery, 159(5), 933-937. https://doi.org/10.1177/0194599818797586

Barr, J. (2015). The anatomist Andreas Vesalius at 500 years old. Journal of Vascular Surgery, 61(5), 1370-1374. https://doi. org/10.1016/j.jvs.2014.11.080

Barsness, K. A., Rooney, D. M., \& Davis, L. M. (2013). Collaboration in simulation: The development and initial validation of a novel thoracoscopic neonatal simulator. Journal of Pediatric Surgery, 48(6), 1232-1238. https://doi. org/10.1016/j.jpedsurg.2013.03.015

Bastawrous, S., Wake, N., Levin, D., \& Ripley, B. (2018). Principles of three-dimensional printing and clinical applications within the abdomen and pelvis. Abdominal Radiology, 43(10), 2809-2822. https://doi.org/10.1007/s00261-018$1554-8$

Bibb, R., Eggbeer, D., \& Paterson, A. (2015). Medical Modelling: The application of advanced design and rapid prototyping techniques in medicine. Elsevier Science \& Technology. http://ebookcentral.proquest.com/lib/vuw/detail. action?docID $=1911168$ 
Bibb, R., \& Winder, J. (2010). A review of the issues surrounding three-dimensional computed tomography for medical modelling using rapid prototyping techniques. Radiography, 16(1), 78-83. https://doi.org/10.1016/j. radi.2009.10.005

Bradley, W. G. (2008). History of medical imaging. Proceedings of the American Philosophical Society, 152(3), 349-361. JSTOR.

Breimer, G. E., Haji, F. A., Bodani, V., Cunningham, M. S., Lopez-Rios, A.-L., Okrainec, A., \& Drake, J. M. (2017). Simulation-based education for endoscopic third ventriculostomy: A comparison between virtual and physical training models. Operative Neurosurgery, 13(1), 89-95. https://doi.org/10.1227/NEU.0000000000001317

Brunton, A., Arikan, C., \& Urban, P. (2015). Pushing the limits of 3d color printing: Error diffusion with translucent materials. ACM Transactions on Graphics, 35(1), 1-13. https://doi.org/10.1145/2832905

Cavaleiro, A., Monteiro, H., \& Calheiros, F. (2008). Training neonatal skills with simulators? Acta Pediatrica, 98(4), 636-639. https://doi.org/10.1111/j.1651-2227.2008.01176

Chen, S. J.-S., Hellier, P., Marchal, M., Gauvrit, J.-Y., Carpentier, R., Morandi, X., \& Collins, D. L. (2012). An anthropomorphic polyvinyl alcohol brain phantom based on Colin27 for use in multimodal imaging. Medical Physics, 39(1), 554-561. https://doi.org/10.1118/1.3673069

Cheng, H., Chen, B. P.-H., Soleas, I. M., Ferko, N. C., Cameron, C. G., \& Hinoul, P. (2017). Prolonged operative duration increases risk of surgical site infections: a systematic review. Surgical Infections, 18(6), 722-735. https://doi. org/10.1089/sur.2017.089

Choonara, Y. E., du Toit, L. C., Kumar, P., Kondiah, P. P. D., \& Pillay, V. (2016). 3D-printing and the effect on medical costs: A new era? Expert Review of Pharmacoeconomics E̊ Outcomes Research, 16(1), 23-32. https://doi.org/10.1586/147 37167.2016 .1138860

Chung, B., \& Park, J. (2019). Real-color volume models made from real-color sectioned images of visible korean. Journal of Korean Medical Science, 34(10). https://doi.org/10.3346/jkms.2019.34.e86

Chung, M., \& Park, J. (2007, July 22). Applications of the Visible Korean Human. In Digital Human Modeling: First International Conference on Digital Human Modeling, Beijing, China.

Cromeens, B. P., Ray, W. C., Hoehne, B., Abayneh, F., Adler, B., \& Besner, G. E. (2017). Facilitating surgeon understanding of complex anatomy using a three-dimensional printed model.Journal of Surgical Research, 216, 18-25. https:// doi.org/10.1016/j.jss.2017.04.003

Crouch, C., \& Pearce, J. (2012). Doing research in design. Bloomsbury Publishing Plc.

Discovery of X-rays. (1967). Nature, 216(5118), 843-843. https://doi.org/10.1038/216843a0 
Doubrovski, E., Tsai, E., Dikovsky, D., Geraedts, J., Herr, H., \& Oxman, N. (2015). Voxel-based fabrication through material property mapping: A design method for bitmap printing. Computer-Aided Design, 60(C), 3-13. https://doi. org/10.2016/j.cad.2014.05.010

Fellner, F. (2016). Introducing cinematic rendering: a novel technique for post-processing medical imaging data. Journal of Biomedical Science and Engineering, 09, 170-175. https://doi.org/10.4236/jbise.2016.93013

Ferretti, A., Schneider, M., \& Blasimme, A. (2018). Machine learning in medicine: opening the new data protection black box. European Data Protection Law Review (EDPL), 4(3), 320-332.

Frayling, C. (1993). Research in Art and Design. Royal College of Art Research Papers, 1(1).

Fusetec 3D Headquarters. (2020). Nasal Swab Clinical Training (Ensuring best practice clinical techniques for unparalleled patient care). 2020 Fusetec 3D. https://media-exp1.licdn.com/dms/document/C4E1FAQFtzwgmvs6-Ng/feedshare-document-pdf-analyzed/0?e=1585692000\&v=beta\&t=zJ1moUb6NkHgAx4Zd9jgMWPwiVHDspHX6gJSe9KjONU

"Getting It Right First Time": Orthopaedic Peer Review Report (pp. 1-62). (2014). British Othopaedic Assosiation. http:// www.wales.nhs.uk/sitesplus/documents/863/3\%20\%28ii\%29\%20Getting\%20It\%20Right\%20First\%20Time.pdf

Ghosh, S. K. (2015). Human cadaveric dissection: A historical account from ancient Greece to the modern era. Anatomy $\Xi^{\circ}$ Cell Biology, 48(3), 153-169. https://doi.org/10.5115/acb.2015.48.3.153

Hamzelou, J. (2015). Virtual human built from more than 5000 slices of a real woman. New Scientist. https://www.newscientist.com/article/mg22730404-000-virtual-human-built-from-more-than-5000-slices-of-a-real-woman/

Hanington, B., \& Martin, B. (2012). Universal methods of design: 100 ways to research complex problems, develop innovative ideas, and design effective solutions. Rockport Publishers.

Health and Disability Commissioner (Code of Health and Disability Services Consumers' Rights) Regulations 1996

Heineman, K. R., Bos, A. F., \& Hadders-Algra, M. (2011). Infant motor profile and cerebral palsy: promising associations. Developmental Medicine E' Child Neurology, 53(s4), 40-45. https://doi.org/10.1111/j.1469-8749.2011.04063.x

Hiller, J., \& Lipson, H. (2009). Design and analysis of digital materials for physical 3D voxel printing. Rapid Prototyping Journal, 15(2), 137-149. https://doi.org/10.1108/13552540910943441

Hochman, J. B., Rhodes, C., Kraut, J., Pisa, J., \& Unger, B. (2015). End user comparison of anatomically matched 3-dimensional printed and virtual haptic temporal bone simulation: A pilot study. Otolaryngology-Head and Neck Surgery, 153(2), 263-268. https://doi.org/10.1177/0194599815586756

Hosny, A., Keating, S., Dilley, J., Ripley, B., Kelil, T., Pieper, S., \& Weaver, J. (2018). From improved diagnostics to presurgical planning: high-resolution functionally graded multimaterial $3 \mathrm{~d}$ printing of biomedical tomographic data sets. 3D Printing and Additive Manufacturing, 5(2), 13-113. https://doi.org/10.1089/3dp.2017.0140 
Huotilainen, E., Jaanimets, R., Valášek, J., Marcián, P., Salmi, M., Tuomi, J., Mäkitie, A., \& Wolff, J. (2014). Inaccuracies in additive manufactured medical skull models caused by the DICOM to STL conversion process. Journal of Cranio-Maxillofacial Surgery, 42(5), e259-e265. https://doi.org/10.1016/j.jcms.2013.10.001

Ionita, C., Mokin, M., Varble, N., Bednarek, D., Xiang, J., Snyder, K., Siddiqui, A., Levy, E., Meng, H., Rudin, S., \& Ionita, C. (2014). Challenges and limitations of patient-specific vascular phantom fabrication using 3D Polyjet printing. Proceedings of SPIE-the International Society for Optical Engineering, 9038, 90380M-90380M. http://search. proquest.com/docview/1826612606/

Jardini, A. L., Larosa, M. A., Filho, R. M., Zavaglia, C. A. de C., Bernardes, L. F., Lambert, C. S., Calderoni, D. R., \& Kharmandayan, P. (2014). Cranial reconstruction: 3D biomodel and custom-built implant created using additive manufacturing. Journal of Cranio-Maxillofacial Surgery, 42(8), 1877-1884. https://doi.org/10.1016/j. jcms.2014.07.006

Kerse, N., \& Dyall, L. (2013). Maori culture integral to health of older Maori. New Zealand Doctor, 29-29.

Kim, P., Choi, C., Han, I., Lee, J., Choi, H., \& Lee, J. (2019). Obtaining informed consent using patient specific 3 d printing cerebral aneurysm model. Journal of Korean Neurosurgical Society, 62(4), 398-404. https://doi.org/10.3340/ jkns.2019.0092

Kirana P. Kumara. (2012). Extracting three dimensional surface model of human kidney from the visible human data set using free software. Leonardo Electronic Journal of Practices and Technologies, 11(20), 115-126.

Kolb, D. (2017). Printing the invisible: Bridging the gap between data and matter through voxel-based 3D printing [Doctoral dissertation]. Massachusetts Institute of Technology.

Kumara, K. (2012). Extracting three dimensional surface model of human kidney from the Visible Human data set using free software. Leonardo Electronic Journal of Practices and Technologies, 11(20), 115-126.

Lim, K. H. A., Loo, Z. Y., Goldie, S. J., Adams, J. W., \& Mcmenamin, P. G. (2016). Use of 3d printed models in medical education: A randomized control trial comparing $3 \mathrm{~d}$ prints versus cadaveric materials for learning external cardiac

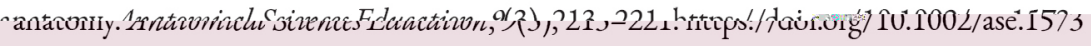

Lin, A. J., Bernstein, J. L., \& Spector, J. A. (2018). Ear reconstruction and 3d printing: is it reality? Current Surgery Reports, 6(2), 4. https://doi.org/10.1007/s40137-018-0198-5

Lioufas, P. A., Quayle, M. R., Leong, J. C., \& McMenamin, P. G. (2016). 3d printed models of cleft palate pathology for surgical education: Plastic and Reconstructive Surgery - Global Open, 4(9), e1029. https://doi.org/10.1097/ GOX.0000000000001029

Loriaux, L. (2016). A Biographical History of Endocrinology. John Wiley \& Sons.

Loukas, M., Hanna, M., Alsaiegh, N., Shoja, M. M., \& Tubbs, R. S. (2011). Clinical anatomy as practiced by ancient Egyptians. Clinical Anatomy, 24(4), 409-415. https://doi.org/10.1002/ca.21155 
Materialise |3D Printing Innovators. (2020). https://www.materialise.com/en

Mavili, M. E., Canter, H. I., Saglam-Aydinatay, B., Kamaci, S., \& Kocadereli, I. (2007). Use of three-dimensional medical modeling methods for precise planning of orthognathic surgery. Journal of Craniofacial Surgery, 18(4), 740-747. https://doi.org/10.1097/scs.0b013e318069014f

McGurk, M., Amis, A. A., Potamianos, P., \& Goodger, N. M. (1997). Rapid prototyping techniques for anatomical modelling in medicine. Annals of The Royal College of Surgeons of England, 79(3), 169-174.

Meurs, W. L. V., Couto, P. M. S., Couto, C. D. S., Bernardes, J. F., \& Ayres-de-Campos, D. (2003). Development of foetal and neonatal simulators at the University of Porto. Medical Education, 37(s1), 29-33. https://doi. org/10.1046/j.1365-2923.37.s1.7.x

Owen, H. (2016). Simulation in Surgery. Simulation in Healthcare Education: An Extensive History (pp. 255-294). Springer International Publishing. https://doi.org/10.1007/978-3-319-26577-3_6

Oxman, N. (2017). Dermi-domus: a grown wardrobe for bodies and buildings. Architectural Design, 87(6), 16-25. https:// doi.org/10.1002/ad.2233

Palter, V. N., \& Grantcharov, T. P. (2010). Simulation in surgical education. Canadian Medical Association. Journal: CMAJ; Ottawa, 182(11), 1191-1196.

Panait, L., Akkary, E., Bell, R. L., Roberts, K. E., Dudrick, S. J., \& Duffy, A. J. (2009). The role of haptic feedback in laparoscopic simulation training. Journal of Surgical Research, 156(2), 312-316. https://doi.org/10.1016/j. jss.2009.04.018

Pommert, A., Höhne, K., Pflesser, B., Richter, E., Riemer, M., Schiemann, T., \& Tiede, U. (2001). Creating a high-resolution spatial/symbolic model of the inner organs based on the Visible Human. Medical Image Analysis, 5(3), 221-228. https://doi.org/10.1016/S1361-8415(01)00044-5

Press Release: Stratasys Responds to COVID-19 Pandemic by Ramping up Production of 3D-Printed Personal Protection Equipment. (2020, March 23). Dow Jones Institutional News. https://search-proquest-com.helicon.vuw.ac.nz/ docview/2381910663?tfr_id=info\%3Axri\%2Fsid\%3Aprimo

Rengier, F., Mehndiratta, A., von Tengg-Kobligk, H., Zechmann, C. M., Unterhinninghofen, R., Kauczor, H.U., \& Giesel, F. L. (2010). 3D printing based on imaging data: Review of medical applications. International Journal of Computer Assisted Radiology and Surgery, 5(4), 335-341. https://doi.org/10.1007/s11548-010-0476-x

Reznek, M., Harter, P., \& Krummel, T. (2002). Virtual reality and simulation: training the future emergency physician. Academic Emergency Medicine, 9(1), 78-87. https://doi.org/10.1197/aemj.9.1.78

Rifkin, B., Ackerman, M., \& Folkenberg, J. (2006). Human Anatomy: Depicting the Body from the Renaissance to Today. Thames \& Hudson Ltd. 
Rizwan I Haque, I., \& Neubert, J. (2020). Deep learning approaches to biomedical image segmentation. Informatics in Medicine Unlocked, 18, 100297. https://doi.org/10.1016/j.imu.2020.100297

Rodriguez Ramirez, E. (2017). A Postgraduate thesis model for research through design based on design criteria. The International Journal of Designed Objects, 11, 11-27. https://doi.org/10.18848/2325-1379/CGP/v11i04/11-27

Rovamo, L., Mattila, M.-M., Andersson, S., \& Rosenberg, P. (2011). Assessment of newborn resuscitation skills of physicians with a simulator manikin. Archives of Disease in Childhood - Fetal and Neonatal Edition, 96(5), F383-F389. https://doi.org/10.1136/adc.2010.194043

Scerbo, M. W., Bliss, J. P., Schmidt, E. A., \& Thompson, S. N. (2006). The efficacy of a medical virtual reality simulator for training phlebotomy. Human Factors, 48(1), 72-84. https://doi.org/10.1518/001872006776412171

Severseike, L., Lee, V., Brandon, T., Bakken, C., \& Bhatia, V. (2019). Polyjet 3D printing of tissue-mimicking materials: How well can 3D printed synthetic myocardium replicate mechanical properties of organic myocardium? BioRxiv, 825794. https://doi.org/10.1101/825794

Sharma, N., Ray, A., Shukla, K., Sharma, S., Pradhan, S., Srivastva, A., \& Aggarwal, L. (2010). Automated medical image segmentation techniques. Journal of Medical Physics, 35(1), 3. https://doi.org/10.4103/0971-6203.58777

Sossou, G., Demoly, F., Belkebir, H., Qi, H., Gomes, S., \& Montavon, G. (2019). Design for 4D printing: A voxel-based modeling and simulation of smart materials. Materials \& Design, 175, 107798. https://doi.org/10.1016/j.matdes.2019.107798. Materials छ Design, ?(?). https://doi.org/10.1016/j.matdes.2019.107798

Stramiello, J. A., Saddawi-Konefka, R., Ryan, J., \& Brigger, M. T. (2020). The role of 3D printing in pediatric airway obstruction: A systematic review. International Journal of Pediatric Otorbinolaryngology, 132, 109923. https://doi. org/10.1016/j.ijporl.2020.109923

Stratasys J750 $0^{T M}$ Digital Anatomy ${ }^{T M} 3 D$-Printer. (2019). Stratasys. https://www.stratasys.com/3d-printers/j750-digital-anatomy

Stute, F., Mici, J., Chamberlain, L., \& Lipson, H. (2018). Digital Wood: 3d internal color texture mapping. 3D Printing and Additive Manufacturing, 5(4), 285-291. https://doi.org/10.1089/3dp.2018.0078

Swetly, T., Kempf, G., Hucke, R., Willing, M., Warkentin, M., \& Stampfl, J. (2016). Bioinspired engineering polymers by voxel-based 3D-printing. BioNanoMaterials, 17(3-4), 145-157. https://doi.org/10.1515/bnm-2015-0021

The National Library of Medicines Visible Human Project. (n.d.). [Digital Library Collections]. Retrieved March 4, 2020, from https://www.nlm.nih.gov/research/visible/visible_human.html

Thielen, M., Delbressine, F., Bambang Oetomo, S., \& Feijs, L. (2019). Anatomically realistic neonatal heart model for use in neonatal patient simulators. Journal of Visualized Experiments, 144. https://doi.org/10.3791/56710

Walsh, K. (2016). Medical Education: A History in 100 Images. CRC Press. https://doi.org/10.1201/b21484 
Watson, J. K., \& Taminger, K. M. B. (2018). A decision-support model for selecting additive manufacturing versus subtractive manufacturing based on energy consumption. Journal of Cleaner Production, 176, 1316-1322. https://doi. org/10.1016/j.jclepro.2015.12.009

Te Kete Ipurangi - New Zealand Government. (n.d.). Well-being, hauora. https://health.tki.org.nz/Teaching-in-HPE/ Health-and-PE-in-the-NZC/Health-and-PE-in-the-NZC-1999/Underlying-concepts/Well-being-hauora

Westwood, J. D. (2002). Medicine Meets Virtual Reality 02/10: Digital Upgrades, Applying Moore's Law to Health. IOS Press.

Wu, Y., Tan, L., Li, Y., Fang, B., Xie, B., Wu, T., \& Zhang, S. (2012). Creation of a female and male segmentation dataset based on Chinese Visible Human (CVH). Computerized Medical Imaging and Graphics, 36(4). https://doi. org/10.1016/j.compmedimag.2012.01.003

Yang, T., Tan, T., Yang, J., Pan, J., Hu, C., Li, J., \& Zou, Y. (2018). The impact of using three-dimensional printed liver models for patient education. Journal of International Medical Research, 46(4), 1570-1578. https://doi. org/10.1177/0300060518755267

Yoo, S.J., Thabit, O., Kim, E. K., Ide, H., Yim, D., Dragulescu, A., Seed, M., Grosse-Wortmann, L., \& van Arsdell, G. (2016). 3D printing in medicine of congenital heart diseases. 3D Printing in Medicine, 2(1), 3. https://doi.org/10.1186/ s41205-016-0004-x

Yoon, S., Park, S., Kang, C., Park, I., Goo, J., \& Kim, Y. (2019). Personalized 3d-printed model for informed consent for stage i lung cancer: a randomized pilot trial. Seminars in Thoracic and Cardiovascular Surgery, 31(2), 316-318. https:// doi.org/10.1053/j.semtcvs.2018.10.017

Young, J. C., Quayle, M. R., Adams, J. W., Bertram, J. F., \& McMenamin, P. G. (2019). Three-dimensional printing of archived human fetal material for teaching purposes. Anatomical Sciences Education, 12(1), 90-96. https://doi. org/10.1002/ase.1805

Zhang, L., Wang, Y., Xiao, M., Han, Q., \& Ding, J. (2008). An ethical solution to the challenges in teaching anatomy with dissection in the Chinese culture. Anatomical Sciences Education, 1(2), 56-59. https://doi.org/10.1002/ase.15 


\subsection{A P P N DIX A}

Appendix A presents the programme for the 3D Technologies in Medicine (3DMED1) conference in Melbourne, Australia, on 14-16 of November, 2019.

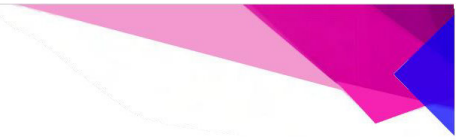

\section{TH ANNUAL 3D Med}

AUSTRALIA CONFERENCE

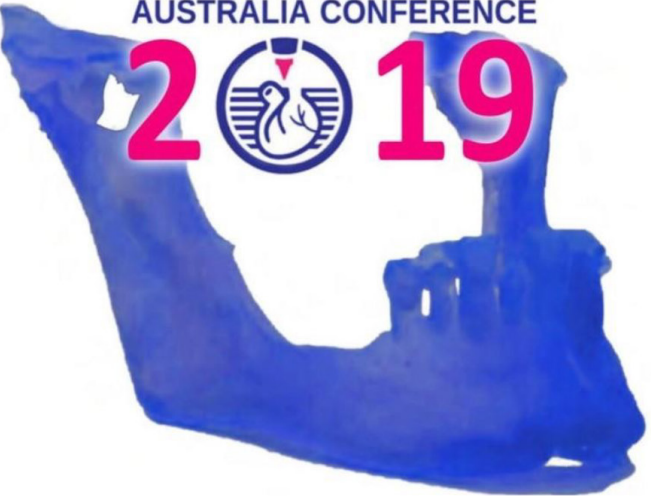

\#3dMed19

$14-16^{\text {th }}$ November 2019
Institutional Partners

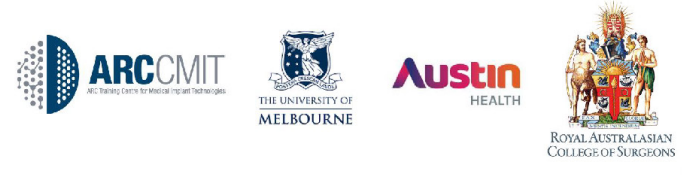

Platinum Sponsors

\section{stratasys}

Silver Sponsors

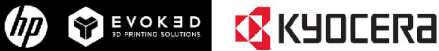

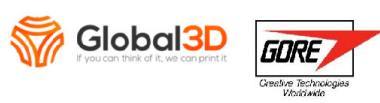

Bronze Sponsors

materialise

Women in 3D Technologies Networking Drinks Sponsor
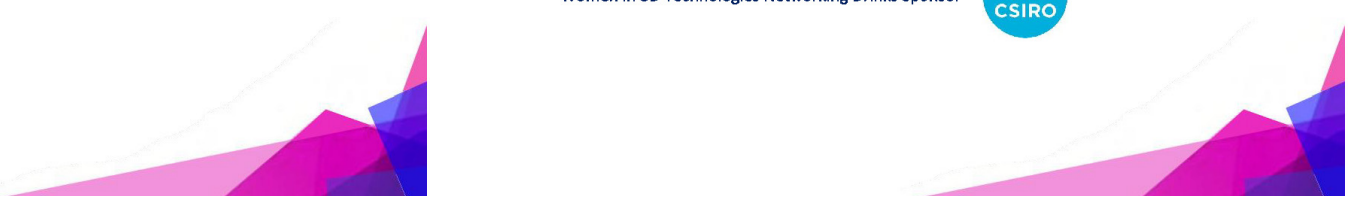
2019 ORGANISING

\section{COMMITTEE}

\section{Conveners}

Jason Chuen

Jasamine Coles-Black

\section{Chairs}

Niranjan Hiremath

Elizabeth Sigston

Blake Cochran

Peter Lee

Felix Sim

Naomi Paxton

Kate Fox

Cathal O'Connell

Darpan Shidid

David Ackland

Payal Mukherjee

Mathilde Desselle

Toan Pham

Ian Chio

Tracie Barber

\section{Abstract Review Committee}

Blake Cochran

Tracie Barber

Mathilde Desselle
VENUES

3D Med Australia 2019 (November 15-16)

AAMI Park

Olympic Blvd

Melbourne VIC 3000

hitps://www aamipark.com.au/arriving-and-access/mapsi

Preconference Workshops (November 14)

Anatomical Modelling Workshop

Electrical Engineering Room 121

Building 193, Parkville Campus

The University of Melbourne

hittps://maps.unimelb.edu.au/parkville/building/193

Stratasys \& Fusetec Workshop - the New $₫ 750$ Digital Anatomy

Technology

Electrical Engineering Room 121

Building 193, Parkyile Campus

https://maps.unimelb.edu.au/parkville/building/193

Women in 3D Technologies Networking Event

Milk the Cow Carlton

323 Lygon St

Carlton VIC 305

https://milkthecow.com.au/pages/locations 


\section{VENUES}

Preconference Tours (November 14)

VR Buffet Tour @ SBS Digital Learning Hub

Room W313, Medical Euilding

Building 181, Parkville Campus

The University of Melbourne

hittps://biomedicalsciences.unimelb.edu.au/studv/dlhitcontact-us

New Experimental Technology (NExT) Lab Tour @ Melbourne Schoo of Design

Glyn Davis Building

Melbourne School of Design

The University of Melbourne

hittos://msd.unimelb.edu.au/contact

BioFab3D Tour @ St Vincent's Hospital Melbourne

Clinical Sciences Building

St Vincent's Hospital Melbourne

29 Regent St

Fitzroy VIC 3065

https://www. biofab3d.ore/

\section{Centre for Additive Manufacturing Lab Tour @ RMIT}

Advanced Manufacturing Precind

RMIT Melbourne City Campus

58 Cardigan St

Carlton VIC 3053

https://www.rmit.edu.au/about/our-locations-and-facilities/facilities/research-

facilities/advanced-manufacturing-precinct/contact-us
Preconference

Workshops and Tours

TIMES AND VENUES

Thursday November 14

\begin{tabular}{|c|c|c|}
\hline 0830-1000 & Anatomical Modelling Workshop & $\begin{array}{l}\text { Electrical Engineering Room } 121 \\
\text { Building } 193 \text {, Parkville Campus } \\
\text { The University of Mel bourne }\end{array}$ \\
\hline $1000-1200$ & $\begin{array}{l}\text { Stratasys \& Fusetec Workshop - the New } \\
\text { J750 Digital Anatomy Technology }\end{array}$ & $\begin{array}{l}\text { Electrical Engineering Room } 121 \\
\text { Building } 193 \text {, Parkville Campus } \\
\text { The University of Melbourne }\end{array}$ \\
\hline $1200-1300$ & Break & \\
\hline $1300-1400$ & VR Buffet @ SBS Digital Learning Hub & $\begin{array}{l}\text { Medical Building } \\
\text { Building } 181 \text {, Parkville Campus } \\
\text { The University of Melbourne }\end{array}$ \\
\hline $1400-1430$ & $\begin{array}{l}\text { NExT Lab Tour @ Melbourne Schaol of } \\
\text { Design }\end{array}$ & $\begin{array}{l}\text { Glyn Davis Building } \\
\text { Melbourne School of Design } \\
\text { Building } 133 \text {, Parkville Campus } \\
\text { The University of Mel bourne }\end{array}$ \\
\hline 1500-1530 & $\begin{array}{l}\text { Biofab3D Tour @ St Vincent's Hospital } \\
\text { Melbourne }\end{array}$ & $\begin{array}{l}\text { Clinical Sciences Building } \\
\text { St Vincent's Hospital Melbourne } \\
29 \text { Regent St } \\
\text { Fitzroy VIC } 3065\end{array}$ \\
\hline $1600-1700$ & $\begin{array}{l}\text { Centre for Additive Manufacturing Lab Tour } \\
\text { @ RMIT }\end{array}$ & $\begin{array}{l}\text { Advanced Manufacturing Precinct } \\
\text { RMIT Mel bourne City Campus } \\
58 \text { Cardigan St } \\
\text { Carlton VIC } 3053\end{array}$ \\
\hline $1700-1800$ & Break & \\
\hline 1800-Late & $\begin{array}{l}\text { Women in 3D Technologies Networking } \\
\text { Drinks (all welcome!) }\end{array}$ & $\begin{array}{l}\text { Milk the Cow Carlton } \\
323 \text { Lygon St } \\
\text { Cariton VIC } 3053\end{array}$ \\
\hline
\end{tabular}
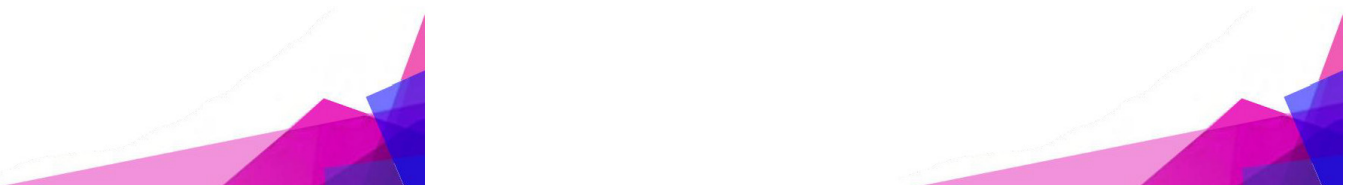
Program Outline

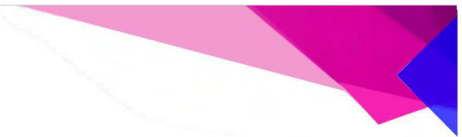

\section{Friday November 15}

\begin{tabular}{|c|c|c|}
\hline 0800-0830 & Registration & Foyer \\
\hline $0830-0845$ & $\begin{array}{l}\text { Frank McGuire MP, Parliamentary Secretary for } \\
\text { Medical Research }\end{array}$ & Lecture Theatre \\
\hline 0845-0900 & $\begin{array}{l}\text { Prof Guy Maddern, Royal Australasian College of } \\
\text { Surgeons }\end{array}$ & Lecture Theatre \\
\hline $0900-1030$ & $\begin{array}{l}\text { Presurgical Planning } \\
\text { Plenary: Jason Chuen }\end{array}$ & Lecture Theatre \\
\hline $1030-1100$ & Morning Tea & Trade Area \\
\hline $1100-1215$ & Cardiovascular Applications & Lecture Theatre \\
\hline $1215-1315$ & Lunch & Trade Area \\
\hline $1315-1445$ & $\begin{array}{l}\text { 3d Visualisation } \\
\text { Keynote: Charles Sevigny }\end{array}$ & Lecture Theatre \\
\hline 1445-1515 & Afternoon Tea & Trade Area \\
\hline \multirow[t]{2}{*}{ 1515-1700 } & Innovation and Industry & Lecture Theatre \\
\hline & Keynote: David Ackland & \\
\hline $1700-1800$ & Quickshot Session 1 & Lecture Theatre \\
\hline
\end{tabular}

\section{Saturday November 16}

\begin{tabular}{|c|c|c|}
\hline $0800-0830$ & Quickshot Session 2 & Lecture Theatre \\
\hline \multirow[t]{3}{*}{ 0830-1030 } & Bioprinting & Lecture Theatre \\
\hline & Plenary: Mia Woodruff & \\
\hline & Keynote: Gordon Wallace & \\
\hline 1030-1100 & Morning Tea & Trade Area \\
\hline $1100-1245$ & Synergy \& Design & Lecture Theatre \\
\hline $1245-1345$ & Lunch & Trade Area \\
\hline $1345-1500$ & Ethics \& Governance & Lecture Theatre \\
\hline $1500-1530$ & Afternoon Tea & Trade Area \\
\hline \multirow[t]{2}{*}{$1530-1800$} & Validation in 3D Technologies & Lecture Theatre \\
\hline & Keynote: Blake Cochran & \\
\hline
\end{tabular}

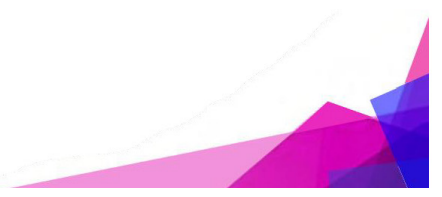

Sessions

Friday November 15: Presurgical Planning

$\begin{array}{lll}\text { Jason Chuen } & \text { Plenary: New Technologies } & 0900-1915 \\ \text { Xiaowei Wang } & \text { 3D FLECT- Fusion Imaging and Emerging Technologies } & 0915-0930 \\ \text { Amy Baker } & \text { Optimising Imaging for 3D Planning } & 0930-0945 \\ \text { Joseph Ischia } & \text { 3D Visualisation in Complex Urooncological Surgery } & 0945-1000 \\ \text { Felix Sim } & \text { 3D Printing Craniofacial Implants } & 1000-1015 \\ & \text { Panel Discussion } & 1015-1030 \\ & \text { Morning Tea } & 1030-1100\end{array}$

\section{Friday November 15: Cardiovascular}

\begin{tabular}{|c|c|c|}
\hline Carmine Gentile & Cardiac Bioprinting & $1100-1115$ \\
\hline Niranjan Hiremath & 3D Printing in Complex Aortic Arch Disease & $1115-1130$ \\
\hline Pujith Vijayaratnam & $\begin{array}{l}\text { 3D Printing Technologies in Experimental Fluid Dynamics } \\
\text { Models of Bridged Arteries }\end{array}$ & 1130-1145 \\
\hline \multirow[t]{3}{*}{$\begin{array}{l}\text { Jasamine Coles- } \\
\text { Black }\end{array}$} & A Technique to Produce Physician Modified Stent Grafts & $1145-1200$ \\
\hline & Panel Discussion & $1200-1215$ \\
\hline & Lunch & 1215-1315 \\
\hline
\end{tabular}

\section{Friday November 15: Visualisation}

$\begin{array}{lll}\text { Charles Sevigny } & \text { Keynote: The Virtual Heart } & 1315-1330 \\
\text { Mathilde Desselle } & \begin{array}{l}\text { No more boring stints in hospitals waiting rooms! } \\
\text { Serious games for patient education }\end{array} & 1330-1345 \\
\text { Ben Loveridge } & \text { VR and Presurgical Planning } & 1345-1400 \\
\text { Michelle Rank } & \begin{array}{l}\text { Bending the knee: can VR rule clinical training } \\
\text { paradigms? }\end{array} & 1400-1415 \\
\text { Andrew Hardidge } & \text { 3D Printed Acetabular Models for Prosthesis Sizing } & 1415-1430 \\
& \text { Panel Discussion } & 1430-1445 \\
& \text { Afternoon Tea } & 1445-1515\end{array}$


Friday November 15

Innovation and Industry

\begin{tabular}{|c|c|c|}
\hline David Ackland & Keynote: Engineering the Human Jaw Joint & $1515-1530$ \\
\hline Mark Roe & $\begin{array}{l}\text { Dissecting Anatomy- An Entrepreneur's } \\
\text { Perspective }\end{array}$ & $1530-1545$ \\
\hline George Dimitroulis & Digital Advances in Maxillofacial Surgery & $1545-1600$ \\
\hline Richard Stratton & Patient's Perspective & $1600-1615$ \\
\hline Karen Reynolds & MDPP-Supporting Medical Innovation & $1615-1630$ \\
\hline \multirow[t]{3}{*}{ Anna Ridgers } & $\begin{array}{l}\text { 3D Printing a Tracheobronchial Tree for } \\
\text { Bronchoscopy Training }\end{array}$ & $1630-1645$ \\
\hline & Panel Discussion & $1645-1700$ \\
\hline & Quickshot Session 1 & $1700-1800$ \\
\hline
\end{tabular}

Sessions

Saturday November 16: Bioprinting

$\begin{array}{lll} & \text { Quickshot Session 2 } & 0800-0830 \\ \text { Mia Woodruff } & \text { Plenary: Directions of Bioprinting } & 0830-0900 \\ \text { Claudia di Bella } & \text { 3D Bioprinting: A Surgeon's Perspective } & 0900-1915 \\ \text { Michael Mueller } & \text { Bioprinting at CSIRO } & 0915-0930 \\ \text { Payal Mukherjee } & \text { The Journey of the Bioprinted Far } & 0930-0945 \\ \text { Behzad Shiroud Heidari } & \text { Bioengineering of Hard-Soft Tissue Interfaces } & 0945-1000 \\ & \text { Panel Discussion } & 1000-1015 \\ \text { Gordon Wallace } & \text { Keynote: Bioprinting } & 1015-1030 \\ & \text { Morning Tea } & 1030-1100\end{array}$

Saturday November 16: Synergy and Design

\begin{tabular}{|c|c|c|}
\hline Icaro Ibanez Arricivita & The Aesthetics of Prosthetics & $1100-1115$ \\
\hline XiaoPeng Li & Implant design & $1115-1130$ \\
\hline Tegan Cheng & $\begin{array}{l}\text { Designing and developing medical devices for } \\
\text { children }\end{array}$ & $1130-1145$ \\
\hline Tom Williamson & $\begin{array}{l}\text { Self-Driving Too Is and Surgical Instrument } \\
\text { Flight: Developing Robotics for the OR }\end{array}$ & $1145-1200$ \\
\hline Toh Yen Pang & Designing Prostheses & $1200-1215$ \\
\hline \multirow[t]{3}{*}{ Mark Midwinter } & $\begin{array}{l}\text { VR and Simulation of Uncommon Time Critical } \\
\text { Medical Interventions }\end{array}$ & $1215-1230$ \\
\hline & Panel Discussion & $1230-1245$ \\
\hline & Lunch & $1245-1345$ \\
\hline
\end{tabular}


Saturday November 16:

Ethics and Governance

$\begin{array}{lll}\text { Cathal O'Connell } & \begin{array}{l}\text { Don't Print Your Heart Cut: The Dangers of } \\ \text { Sensationalism in Science }\end{array} & 1345-1400 \\ & \begin{array}{l}\text { Department of Health and Human Services - } \\ \text { Paul Fennessy }\end{array} & \\ & \begin{array}{l}\text { An Evidence Review of the Surgical } \\ \text { Afplications of 3D Printing }\end{array} & 1400-1415 \\ \text { John Skerritt } & \begin{array}{l}\text { TGA- Regulatory Reforms to Personalised } \\ \text { Kate Fox }\end{array} & 1415-1430 \\ & \text { Medical Devices } & 1430-1445 \\ & \text { The Fun Patent Talk } & 1445-1500 \\ & \text { Panel Discussion } & 1500-1530\end{array}$

Saturday November 16: Validation

$\begin{array}{lll}\text { Blake Cochran } & \text { Keynote: 3D Printing Awesomeness } & 1530-1545 \\ \text { Kate Dunn } & \text { 3D Printed Surgical Simulation and Haptics } & 1545-1600 \\ \text { Peter Lee } & \begin{array}{l}\text { ARC Training Centre for Medical Implant } \\ \text { Technologies: Euiding Mutidisciplinary }\end{array} & 1615-1630 \\ \text { Karel Habig } & \text { Teams and Projects } & \\ \text { Atandrilla Das } & \text { 3D Printing in Helicopter Retrieval } & 1630-1645 \\ \text { Natharniel McTaggart } & \text { 3D Visual sation in Robotic Surgery } & 1645-1700 \\ \text { Stewart Ryan } & \text { Starting a 3D Printing Service at Auckland City } & 1700-1715 \\ & \text { Hospital } & 1715-1730 \\ & \text { 3D Printing in Veterinary Education } & 1730-1745 \\ & \text { Panel Discussion } & 1745-1800\end{array}$

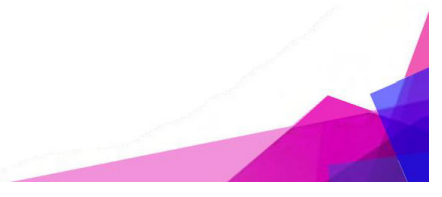




\subsection{A P P E N D IX B}

Ethics documents are presented in Appendix B. These documents allow the researcher to converse and receive comments from industry experts.

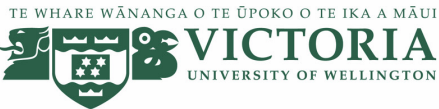

\section{Professional perspective, gaining an expert opinion from industry}

\section{INFORMATION SHEET FOR PARTICIPANTS}

Thank you for your interest in this project. Please read this information before deciding whether or not to take part. If you decide to particinate. thank vou. If vou decide not to take part thank you for considering my request.

\section{Who am I?}

My name is (Student add name) and I am a Masters student in the Masters of Design Innovation Programme at Victoria University of Wellington. This research project is work towards my thesis and design composition.

\section{What is the aim of the project?}

This project is part of the MADE ${ }^{1}$ Research Group that is focused on the design of products and services that may have commercial potential or are allied to industry.

During the design process it is beneficial to evaluate ideas and theories by gaining an expert opinion. This acknowledges the intuitive awareness professionals have and provides a balance in any scholarly critique.

This research has been approved by the Victoria University of Wellington Human Ethics Co 324294

\section{How can you help?}

If you agree to take part I will meet with you at your Company or the University. I will present my design work that may include models, images and/or video, I would then appreciate your feedback based on your professional opinion and the following question. 'How does this academic design research relate to the industry and how might it be applied commercially or conceptually within your company or the field in general?'

The presentation will take no more than 30 minutes and I would like to audio record your response with your permission and write up notes later.

\section{What will happen to the information you give?}

${ }^{1}$ MADE http://made.ac.nz/about-made/

Multi-property Additive-manufacturing Design Experiments - MADE - is the concerted manifestation of 15 years of research and knowledge at Victoria University of Wellington (New Zealand) into emerging digital technologies specifically $3 D$ printing - and its commitment to educating students for a changing and challenging new world. 
The information you provide as feedback will be confidential until you consent to publication. If you require (prior to any publication) I will provide you with a proofing document that clearly shows what I wish to publish and how you and your company may be referenced, this will require your and your organisations consent (provided you have the authority to agree to this on behalf of the organisation). Only my supervisors and I will read the meeting notes. Audio recordings will be kept securely and destroyed 1 year after the research ends.

\section{What will the project produce?}

The information from my research will be used in Masters Thesis and/or academic publications and conferences. Having Industry perspectives will assist in developing research relationships that foster critique of academic and professional practice.

If you accept this invitation, what are your rights as a research participant?

You do not have to accept this invitation if you don't want to. If you do decide to participate, you have the right to:

- ask for the recorder to be turned off at any time during the interview

- ask any questions about the study at any time

- read over (comment as required) a proofing document and consent to publication

- be able to read any reports of this research by emailing the researcher to request a copy.

If you have any questions or problems, who can you contact?

If you have any questions, either now or in the future, please feel free to contact either:

\section{Student:}

Name:

University email address:

(Note: students should not provide personal cell phone numbers)

\section{Supervisor:}

Name: Bernard Guy

Role: Lecturer

School: Design

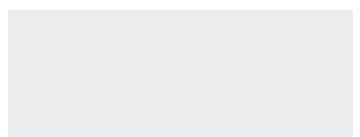

\section{Human Ethics Committee information}

If you have any concerns about the ethical conduct of the research you may contact the Victoria University HEC Convenor: Dr Judith Loveridge, email 


\section{Professional perspectives, gaining an expert opinion from industry}

\section{CONSENT TO INTERVIEW}

This consent form will be held for 3 years.

Researcher: (Student Add Name), School of Design, Victoria University of Wellington.

- I have read the Information Sheet and the project has been explained to me. My questions have been answered to my satisfaction. I understand that I can ask further questions at any time.

- I agree to take part in an audio recorded interview.

I understand that:

- The information I have provided will be destroyed 1 year after the research is finished.

- Any information I provide will be kept confidential to the researcher and the supervisor

- I understand that the results will be used for a Masters Thesis and/or academic publications and/or presented to conferences.

- I consent to information or opinions which I have given being attributed

to me/my organisation in any reports on this research and have the Yes $\square$ No $\square$ authority to agree to this on behalf of the organisation:

Or

- I would like to review a proofing document prior to any publication. $\quad$ Yes $\square$ No $\square$

- $\quad$ I would like to receive a copy of the final report and have added my email Yes $\square$ No $\square$ address below.

Signature of participant:

Name of participant:

Date

Contact details: 


\section{BENEATH THE SKIN}

by

A N A MOR R I S

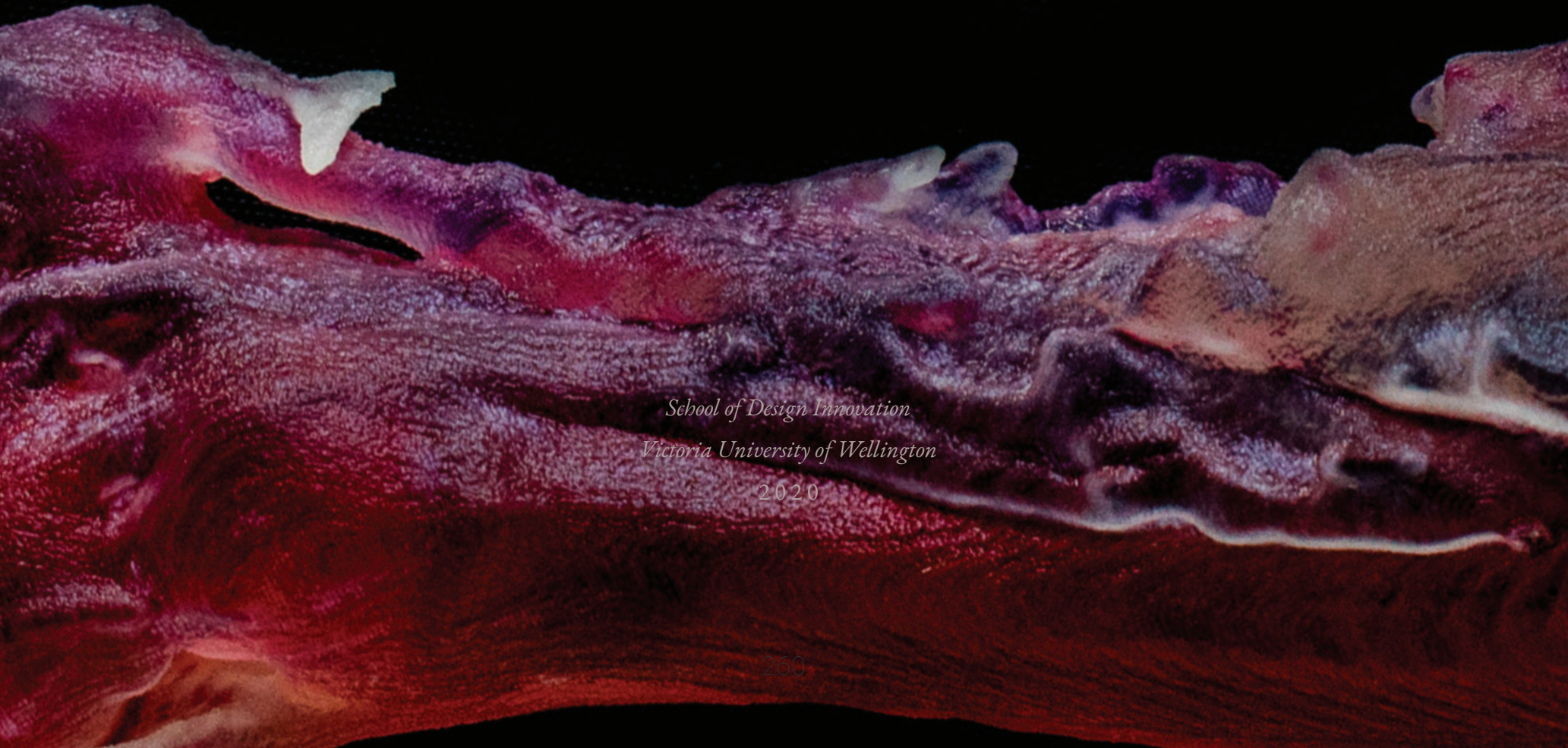

